\title{
EXPERIMENTAL IMAGING OF ASTHMA PROGRESSION AND THERAPEUTIC RESPONSE IN MOUSE LUNG MODELS
}

a dissertation presented

by

\section{Christian Dullin}

\author{
to \\ the Institute for Diagnostic and Interventional Radiology \\ University Medical Center Goettingen \\ in partial fulfillment of the requirements \\ for the degree of \\ Dr. sci. hum. \\ in the subject of \\ „Medizinische Humanwissenschaften“ \\ University Medical Center Goettingen
}

28 July 2015 
SUPERVISOR

SECOND THESIS COMMITTEE MEMBER

THIRD THESIS COMMITTEE MEMBER

FOURTH THESIS COMMITTEE MEMBER
Prof. Dr. rer. nat. Walter Stühmer

Head of the Dept. of "Molecular Biology of Neuronal Signals"

Max Planck Institute for Experimental Medicine

Prof. Dr. med. Frauke Alves

Group leader "Translational Molecular Imaging in Oncology “

Max Planck Institute for Experimental Medicine, Dept. of Hematology and Medical Oncology and Institute for Diagnostic and Interventional Radiology, University Medical Center Goettingen

Senior-Prof. Dr. Eberhard Fuchs

German Primate Center, Goettingen

Prof. Dr. med. Holger Reichardt

Professor of Experimental Immunology,

Institute for Cellular and Molecular Immunology, University Medical Center Goettingen 


\section{DECLARATION}

Herewith I declare that this thesis entitled "Experimental imaging of asthma progression and therapeutic response in mouse lung models", is my original work and has been written using only the sources quoted.

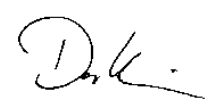

Christian Dullin

Göttingen, 20 October 2015 


\section{ABSTRACT}

Mouse models have been a versatile tool in asthma research, however the application of imaging techniques to quantify hallmarks of asthma, to follow the course of the disease or to monitor treatment response are hampered by the small size of the mouse lung. Moreover, breathing causes substantial motion artifacts in most of the in-vivo imaging methods. In addition, the air-tissue interfaces within the lung causes scattering effects in optical imaging applications, the large cavity of the lung leads to susceptibility artifacts in magnetic resonance imaging and the rib cage hinders the use of medical ultrasound by reflecting the sound waves.

Therefore, novel imaging strategies to study asthma-related anatomical, functional and molecular alterations within the mouse lung are in great demand. In order to overcome the obstacles in lung imaging of asthmatic mice we followed three main routes: A) anatomical imaging by inline free propagation Synchrotron phase contrast CT to provide a detailed three-dimensional depiction of the lung morphology in order to assess and quantify asthma related alterations and to track barium sulfate filled macrophages, B) direct measurement of lung function utilizing low-dose planar cinematic x-ray imaging and C) functional imaging by means of near infrared optical imaging in combination with labeled antibodies or smart probes activated in the presence of inflammation.

By developing and applying phase contrast CT for anatomical imaging I was able to quantify morphological alterations while measuring the soft tissue to air ratio, narrowing of the airways as well as bronchi wall thickening within asthmatic lung tissue and therefore to discriminate between mice from asthma models of different severity, treated mice and healthy controls. Moreover, this imaging technique allowed the tracking of barium sulfate filled macrophages that were intratracheally applied into mouse lungs. This allowed for the first time the combination of functional imaging with detailed morphological three-dimensional analysis of asthmatic mouse lungs under in vivo like conditions and in great detail $(9 \mu \mathrm{m})$. In order to correlate these results with the grade of dyspnea in living mice, I invented a simple and reliable method to assess lung function by utilizing planar cinematic low-dose x-ray imaging. Using this approach I was able to measure differences in the lung function of asthmatic, treated and healthy mice in vivo over time. Moreover, I demonstrated that the results obtained by this new approach correlate with CT and histology. Furthermore, this method has the potential to be applied on free moving un-anesthetized mice, which would lower the stress of the mouse during lung function measurements and would thereby generate more reliable data. Using near infrared fluorescence imaging we demonstrated that we are able to monitor different molecular hallmarks of asthma in vivo over time. First, the use of a novel dendritic polyglycerol sulfate dye (MN2012), which targets selectins, allowed us to depict differences in the grade of inflammation between asthmatic mice and healthy controls. Moreover, we proved that this novel probe expresses a faster kinetic and a higher specificity than state-of-theart commercial probes. Second, we showed that by using a specific antibody-dye-conjugate targeting siglecF predominately expressed on eosinophils, eosinophilia in asthmatic mice can be imaged. Third near infrared fluorescence imaging allowed to determine the fate of inhaled fluorescent nanoparticles within the lung that were taken up by macrophages. All these methods were cross-validated and verified by histology and near infrared fluorescence microscopy. In summary, the imaging strategies developed in the here presented thesis establishing an imaging platform for assessing asthma mouse models, which can now be used to study specific effects in asthma models of different severity, to follow the course of the disease or to monitor treatment response. 


\section{ZUSAMMENFASSUNG}

Asthma ist eine Erkrankung die das komplette Immunsystems involviert, ein System so komplex, dass es sich nur unzureichend in-vitro studieren lässt. Daher haben sich Mausmodelle als ein unverzichtbares Werkzeug in der präklinischen Asthmaforschung etabliert. Da es sich weiterhin bei Asthma um eine Erkrankung handelt, die durch eine schnelle Änderung der Symptome gekennzeichnet ist, wäre longitudinale vorzugsweise nicht-invasive Bildgebung, insbesondere bei der Entwicklung und Bewertung neuer Therapiekonzepte von großem Interesse. Nachteilig hingegen ist, dass die Darstellung der Mauslunge in der Praxis auf Grund der Größe des Organs und, im Falle einer in vivo Bildgebung, durch die Bewegung des Brustkorbes sich als äußerst schwierig herausstellt. Die Vielzahl der Luft-Gewebe-Grenzflächen erzeugt starke Streuung in der optischen Bildgebung, der große Hohlraum der Lunge verursacht Suszeptibilitätsartefakte bei der MRT und die Rippen erschweren eine Ultraschallbildgebung.

Aus diesen Gründen besteht ein großer Bedarf an neuen Bildgebungsverfahren, um die durch Asthma verursachten anatomischen, funktionalen und molekularen Veränderungen darstellen zu können. Um die Schwierigkeiten in der Lungenbildgebung bei Mäusen zu umgehen, habe ich mich auf drei wesentliche Bildgebungsstrategien fokussiert: A) anatomische Bildgebung durch "inline free propagation phase contrast computed tomography", B) direkte Messung der Lungenfunktion durch “low dose planar cinematic x-ray imaging” und C) funktionale Bildgebung mit Hilfe der „near infrared fluorescence imaging" in Kombination mit Antikörpern, die mit einem Fluoreszenzfarbstoff markiert wurden, oder "smart probes", die in Gegenwart von Entzündungen aktiviert werden.

Durch die Anwendung von "phase contrast computed tomography" für die anatomische Bildgebung war ich in der Lage morphologische Veränderung des Lungengewebes zu quantifizieren, indem ich lokal das Verhältnis zwischen Weichgewebe und Luft, das Zusammenziehen der Luftwege sowie das Anschwellen der Bronchialwände im asthmatischen Lungengewebe ausgewertet habe. Diese Parameter erlaubten es zwischen Mäusen von Asthmamodellen unterschiedlicher Schweregrade, therapierten und gesunden Mäusen zu unterscheiden. Zusätzlich ermöglichte diese Technik die Darstellung intra-tracheal applizierter Bariumsulfat markierter Makrophagen im Lungengewebe. Dies stellt meines Wissens die erste Kombination einer funktionalisierten Kontrastierung und hochauflösender Lungenbildgebung mittels CT unter in vivo ähnlichen Bedingungen dar. Um diese Ergebnisse mit dem Grad der asthmabedingten Kurzatmigkeit zu korrelieren, habe ich eine einfache und verlässige Methode entwickelt die es, basierend auf 2D Röntgen-videos niedriger Röntgendosis ( $\sim 6,5 \mathrm{mGy})$ erlaubt, in narkotisierten Mäusen die Lungenfunktion zu bewerten. Mit Hilfe dieser neuen Methode gelang es mir charakteristische Unterschiede in der Lungenfunktion von asthmatischen, therapierten und gesunden Mäusen in vivo über die Zeit nachzuweisen, und diese Resultate mit den Ergebnissen von CT und Histologie zu korrelieren. Das Verfahren wird derzeit von mir für die Anwendung an frei beweglichen und nicht narkotisierten Mäusen weiterentwickelt. Dies sollte zu einer deutlichen Stressreduktion für die Maus bei der Untersuchung führen und somit, vor allem in Asthma, im Gegensatz zu etablierten Verfahren wie Plethysmographie, die Erhebung validerer Messdaten erlauben. Mit Hilfe von „near infrared fluorescence imaging“ konnten wir in vivo und longitudinal erfolgreich verschiedene durch Asthma ausgelöste molekulare Veränderungen in der Mauslunge verfolgen. Erstens erlaubte die Verwendung einer neuen Polyglyzerol Probe mit dendritischer Struktur (MN2012) die spezifisch an Selektine bindet, die Darstellung der durch Asthma verursachten Entzündung der Lunge. Im Zuge dessen konnten wir nachweisen, dass sich 
MN2012 zur Darstellung von Enzymkinetiken bei Entzündungsreaktionen durch eine schnellere Kinetik und höher Spezifität als kommerziell erhältliche Proben auszeichnet. Zweitens haben wir gezeigt, dass in Kombination mit einem Fluoreszenz markiertem Antikörper gegen SiglecF, einem Antigen das hauptsächlich auf Eosinophilen exprimiert ist, Eosinophilie in asthmatischen Mäusen verfolgt und der Effekt einer Dexamethason Behandlung ebenso dargestellt werden kann. Drittens konnten wir den Verbleib inhalierter fluoreszierender Nanopartikel in der Lunge der Maus in vivo untersuchen und dabei nachweisen, dass diese hauptsächlich von endogenen Makrophagen im Lungengewebe aufgenommen werden. Alle diese Techniken wurden gegeneinander und mittels histologischer Analyse und Fluoreszenzmikroskopie korreliert und validiert.

Zusammenfassend bilden die in meiner Dissertation entwickelten Lungenbildgebungsstrategien für Asthmamausmodelle eine Bildgebungsplattform, um sowohl spezifische Effekte in asthmatischen Mäusen unterschiedlichen Schweregrades als auch die Auswirkungen neuer Therapien abzubilden und im Detail zu untersuchen. 
TABLE OF CONTENT

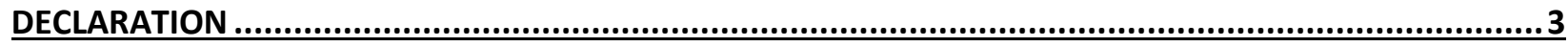

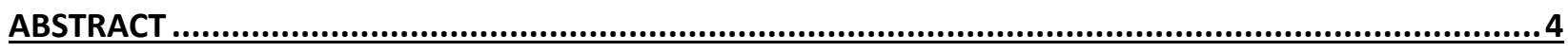

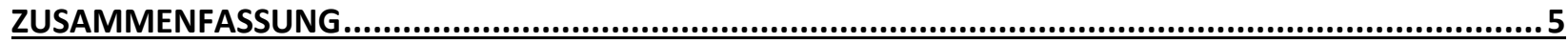

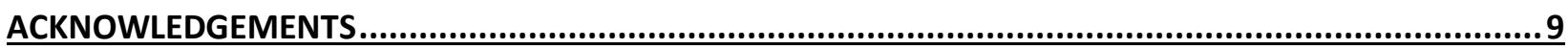

DEDICATION $\ldots \ldots \ldots \ldots \ldots \ldots \ldots \ldots \ldots \ldots \ldots \ldots \ldots \ldots \ldots \ldots \ldots \ldots \ldots \ldots \ldots \ldots \ldots \ldots \ldots \ldots \ldots \ldots \ldots \ldots \ldots \ldots \ldots \ldots \ldots \ldots \ldots \ldots \ldots 11$

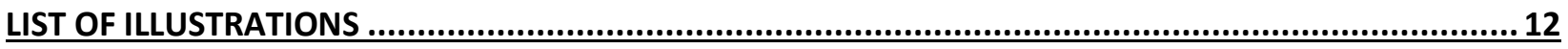

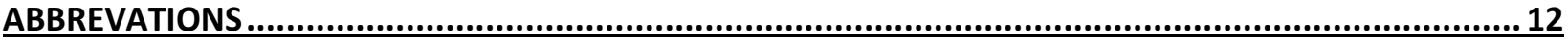

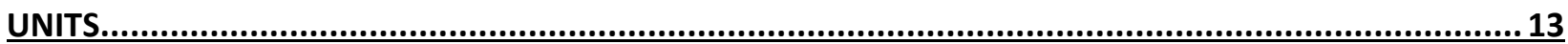

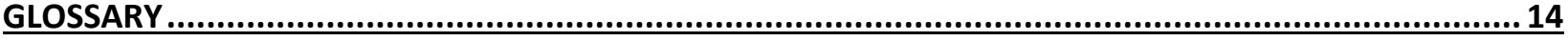

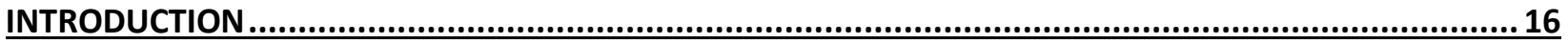

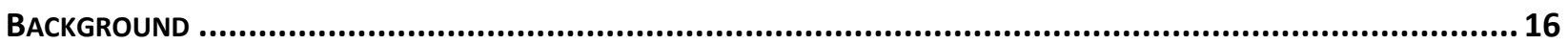

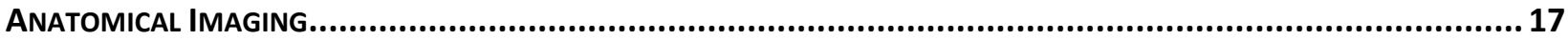

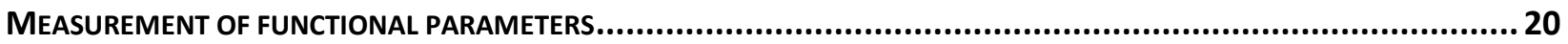

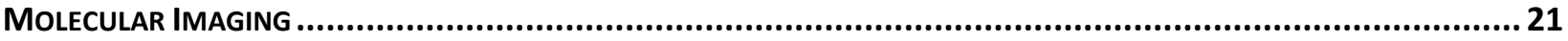

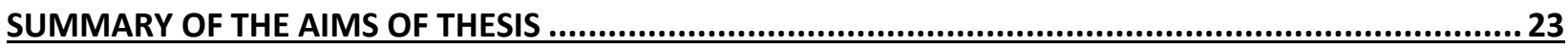

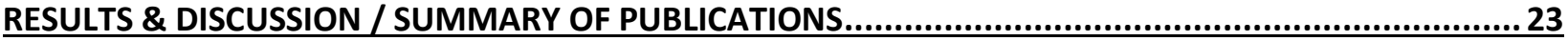

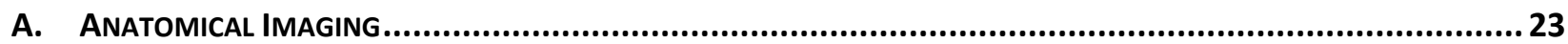

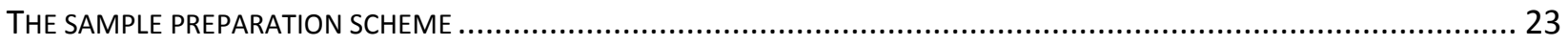

SINGLE DISTANCE PHASE RETRIEVAL - THE METHOD OF CHOICE FOR IN-SITU LUNG IMAGING .................................... 24

1) ACCURACY AND PRECISION OF RECONSTRUCTION OF COMPLEX REFRACTIVE INDEX IN NEAR-FIELD SINGLE-DISTANCE

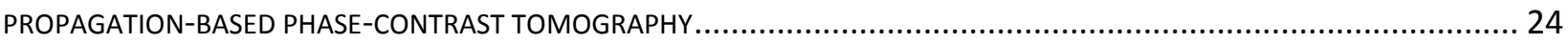

2) QUANTITATIVE EVALUATION OF A SINGLE-DISTANCE PHASE-RETRIEVAL METHOD APPLIED ON IN-LINE PHASE-CONTRAST IMAGES OF A MOUSE LUNG .

3) PhASE CONTRAST CT FOR QUANTIFICATION OF STRUCTURAL CHANGES IN LUNGS OF ASTHMA MOUSE MODELS OF DIFFERENT SEVERITY

4) FUNCTIONALIZED SYNCHROTRON IN-LINE PHASE-CONTRAST COMPUTED TOMOGRAPHY: A NOVEL APPROACH FOR SIMULTANEOUS QUANTIFICATION OF STRUCTURAL ALTERATIONS AND LOCALIZATION OF BARIUM-LABELED ALVEOLAR

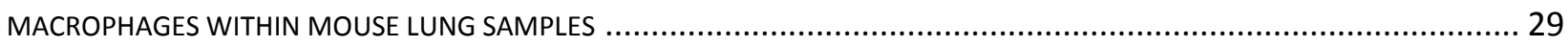

5) PHASE CONTRAST ZOOM-TOMOGRAPHY REVEALS DETAILED LOCATION OF MACROPHAGES IN MOUSE LUNGS ............ 31

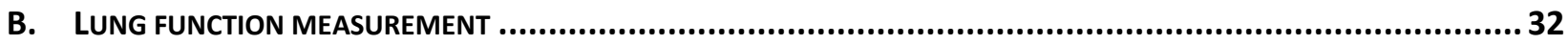

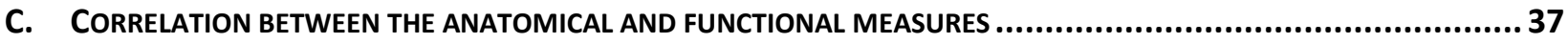

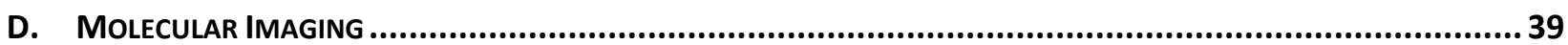

1) Dendritic Polyglycerolsulfate Near Infrared Fluorescent (NIRF) Dye Conjugate for Non-Invasively Monitoring of Inflammation in AN Allergic Asthma Mouse Model. 


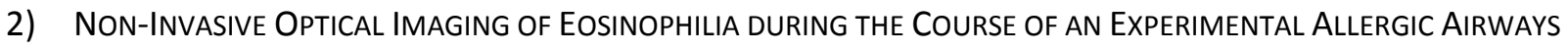
DISEASE MODEL AND IN RESPONSE TO THERAPY

3) TRACKING OF INHALED NEAR-INFRARED NANOPARTICLES IN LUNGS OF SKH-1 MICE WITH ALLERGIC AIRWAY INFLAMMATION

SUMMARY OF THE MOLECULAR IMAGING PART .

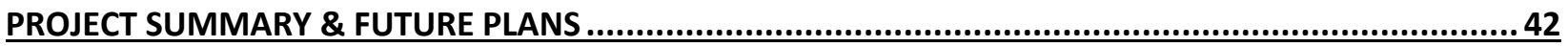

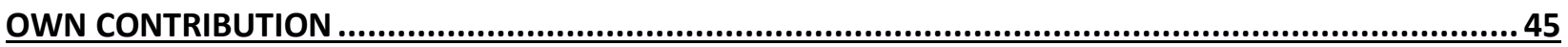

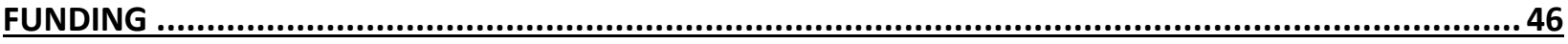

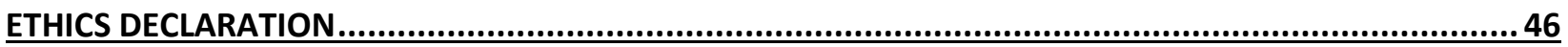

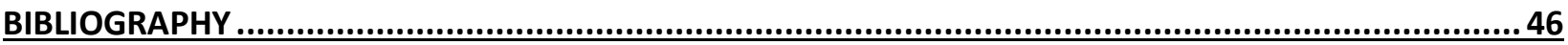

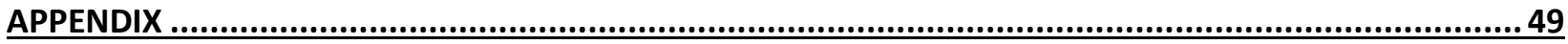

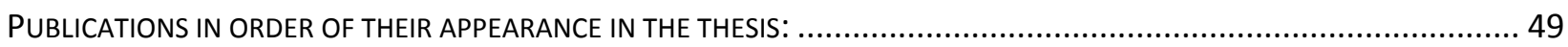

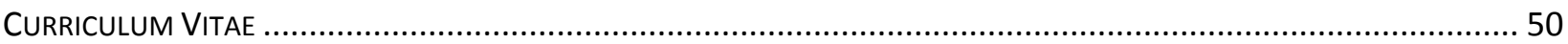




\section{ACKNOWLEDGEMENTS}

AG Alves I would like to thank all the current and former scientists and technicians of the interdisciplinary research group of Prof. Frauke Alves. The knowledge and experience I gained in the field of preclinical imaging in the past ten years while working in her group has been the foundation of the work presented here. First, I would like to mention especially the excellent technical assistances from Sarah Greco, Bärbel Heidrich and Roswitha Streich, who ensured the success of the experiments. I also like to thank Dr. Andrea Markus, who not only helped me to improve my English in many publications. She was always a source of fruitful discussions and has therefore in many ways contributed to the results of the here presented thesis. Finally, I would like to thank Prof. Frauke Alves who not only started the animal imaging projects, but also created an interdisciplinary group, which despite being quite small, can compete with the big international and leading groups in the imaging field. I am very grateful that she always gave me the space to try out some unorthodox ideas and showed an interest in my "physics" point of view. The contact to the SYRMEP Synchrotron group in Italy, now to be seen as the major inspiration in my current research, was based on a European project that she initiated. In summary I can say that Prof. Alves has profoundly influenced my whole scientific live and I look forward to the future projects with her group.

Institute of Diagnostic and Interventional Radiology

This institute has been my working place for over a decade and I thank all the colleagues I had the pleasure to work with. Especially, I thank Prof. Joachim Lotz, the head of the institute for giving me the opportunity to follow my own ideas in the field of imaging. Moreover, I thank my assistant Christina Malowsky, who has helped a lot in performing the experiments, analyzing the data and coordinating the project. Furthermore, I like to thank the former head of the department Prof. Eckardt Grabbe whose vision made it possible to start an imaging group and who had the courage to put a "nameless" young physicist in charge of these extremely precious research imaging devices.

SYRMEP To get to know the team of the beamline "Synchrotron Radiation for Medical Physics" at the Italian Synchrotron Light Source "Elettra" in Trieste was by far the best experience in my research career. Not only the exciting technical capabilities of the beamline, but also the very collaborative nature of the entire group in Trieste has led to the success of the experiments presented in this thesis. I thank a former colleague of the SYRMEP group, Sara Mohammadi, who evaluated the potential of phase contrast CT imaging in mouse lung imaging and therefore helped to form the basis of the here presented thesis. I like to thank my friend Emanuel Larsson, who spent many nights with me at that particular beamline measuring biological samples. He has supported my work by analyzing the data sets and contributed to planning and design of the experiments.

Especially, I like to thank Dr. Giuliana Tromba, who supervised all my Synchrotron 
experiments, discussed my experiments, publications and ideas countless times with me. She inspired me to go on with Synchrotron imaging and made me feel part of a family rather than an experimental imaging group.

Prof. Tim Gureyev, Monash University, Melbourne, Australia

Dr. Timur Gureyev former member of CSIRO (Australia) has been a very valuable discussion partner. His work, especially in phase retrieval, was of critical importance for the developed image processing scheme and therefore essential for this thesis.

Max Plank Institute for Experimental Medicine

The group "Molecular Biology of Neuronal Signals" of the Max Planck Institute for Experimental Medicine headed by Prof. Walther Stühmer is the main partner of the translational and interdisciplinary imaging group since the beginning. Every time I needed help, support, resources or simply an environment in which I could focus on science, Prof. Stühmer and his group provided all this non-bureaucratically and fast and I'm very grateful for all this support.

Institute for X-ray Physics

While working on this study I came in contact with the x-ray physics institute of the University Goettingen headed by Prof. Tim Salditt. His PhD student, Martin Krenkel has helped me to analyze further the fate of the labeled macrophages within the lung by performing phase contrast imaging with stunning resolution. These studies are now the basis for further collaborations and have already led to more exciting experiments.

CBM The molecular imaging group at the "Cluster in Biomedicine" in Trieste was a partner in the European project "Public Private Partnership for Asthma Genomics and Imaging". CBM was my working place for more than eight months in the past years. All the colleagues but most of all Dr. Simeone dal Monego and Andrea Lorenzon have contributed to data acquisition and processing as well as optimized the lung sample preparation scheme that is now used for all in-situ lung studies. Besides that they made me enjoy my stays in Trieste a lot.

Prof. Steven Kilfeather, Aiertec

Prof. Kilfeather has the skill to explain the complex mechanism of asthma in a way even a physicist understands. The things he taught me have helped a lot to interpret the results obtained with the different imaging techniques. 


\section{DEDICATION}

My thesis is dedicated to Prof. Werner Haubenreißer. He was a renowned theoretical physicist and my Grandfather, who has inspired me to enter the world of science and physics in particular. I am very proud to follow in his footsteps and I know he would be proud too. 


\section{LIST OF ILLUSTRATIONS}

FiguRE 1WORLD MAP OF PREVALENCE OF CLINICAL ASTHMA [2].

FIGURE 2 OVERVIEW OF ASTHMA HALLMARKS WHICH PRESENTS POTENTIAL TARGETS FOR IMAGING DISTRIBUTED IN MOLECULAR,

FUNCTIONAL AND ANATOMICAL PARAMETER.

FIgURE 3 CROSS SECTIONS THROUGH LUNG SCANS ACQUIRED WITH EXPLORE LOCUS SP MICROCT DEMONSTRATE A HIGH NOISE LEVEL:... 19

FIGURE 4 IMPACT OF THE PHASE SHIFT ON THE IMAGE FORMATION WITH INCREASING SAMPLE-TO-DETECTOR DISTANCE: ..................... 20

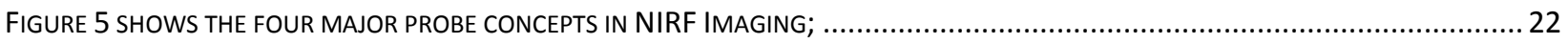

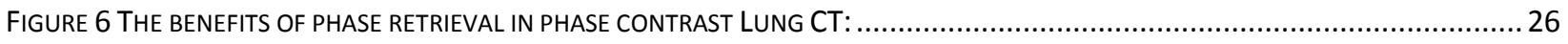

FIGURE 7 SCHEDULE OF THE DIFFERENT OVA INDUCED ALLERGIC AIRWAY INFLAMMATION MOUSE MODELS USED: ............................27

FIGURE 8 DEMONSTRATION OF THE IMAGE QUALITY INLINE PHASE CONTRAST CT OF IN-SITU MOUSE LUNGS AFTER PHASE RETRIEVAL:....... 28

FIGURE 9: 3D LOCALIZATION OF THE BARIUM SULFATE LABELLED MACROPHAGES WITHIN THE LUNG: .......................................... 31

FIGURE 10: 3D LOCALIZATION OF THE LABELLED MACROPHAGES IN A RECONSTRUCTED VOLUME OF A THICK LUNG SECTION SCANNED BY

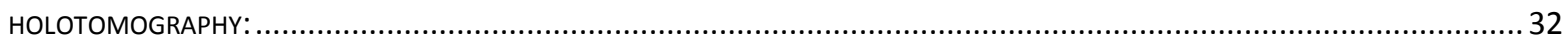

FIGURE 11 PRINCIPLE OF THE IN-VIVO LUNG FUNCTION MEASUREMENT APPROACH BASED ON CINEMATIC PLANAR X-RAY IMAGING: ......... 34

FIGURE 12 RESULTS OF THE DIFFERENT LUNG FUNCTION PARAMETERS QF, C AND L FOR THE TWO ASTHMA MODELS, CONTROLS AND DEX

TREATED MICE:

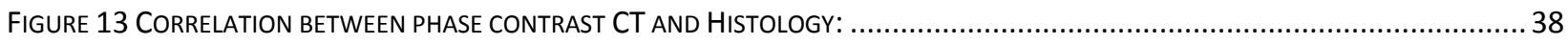

FIGURE 14 RELATIONSHIP BETWEEN PHASE CONTRAST CT (VOL.RATIO) AND THE LUNG FUNCTION PARAMETER (QF, C AND L[S]):........... 39

\section{ABBREVATIONS}

2D

3D

AM

AnIso

$\mathrm{AuC}$

BAL

C

CCD

CNR

COV

CT

DEX

DiD

EEI

FBP

FoM

GINA two-dimensional

three-dimensional

alveolar macrophage

anisotropy index

area under the curve

broncho-alveolar lavage

constant (of the lung function fitting polynomial function)

charged-coupled device

contrast-to-noise ratio

coefficient of variation

computed tomography

dexamethasone

Vibrant cell labelling solution (absorption 644nm, emission 665nm)

edge index

filtered back projection algorithm

figure-of-merit

Global Initiative against asthma 


$\begin{array}{ll}\text { H\&E } & \text { haematoxylin \& eosin } \\ \text { i.p. } & \text { intraperitonial } \\ \text { i.n. } & \text { intranasal } \\ \text { L } & \text { length of the breathing event } \\ \text { LPS } & \text { lipopolysaccharide } \\ \text { MAA } & \text { mild acute asthma } \\ \text { MCN } & \text { control of the mild asthma model } \\ \text { MH-S } & \text { an immortalized alveolar macrophage cell line } \\ \text { MIF } & \text { macrophage inhibition factor } \\ \text { MRI } & \text { magnet resonance imaging } \\ \text { NIR } & \text { near infrared } \\ \text { NIRF } & \text { near infrared fluorescence } \\ \text { OVA } & \text { ovalbumin } \\ \text { PAS } & \text { periodic acid-schiff } \\ \text { PBS } & \text { phosphate buffered saline } \\ \text { PET } & \text { positron emission tomography } \\ \text { QF } & \text { quadratic factor (of the lung function fitting polynomial function) } \\ \text { ROI } & \text { region of interest } \\ \text { SAA } & \text { severe acute asthma } \\ \text { SCN } & \text { control of the severe asthma model } \\ \text { STD } & \text { standard deviation } \\ \text { SPECT } & \text { single photon emission computed tomography } \\ \text { VOI } & \text { volume of interest } \\ \text { XAF } & \text { x-ray attenuation function } \\ & \end{array}$

\section{UNITS}

Gy gray measures the dose of a specific radiation source, meaning the work that is performed by the radiation within the object. 1Gy is defined as $1 \mathrm{~J}$ (Joule) of radiation energy absorbed by $1 \mathrm{~kg}$ of matter

$\mathrm{eV}$ electron volt a measure of energy, mainly used for accelerated electrons. Defined as the energy of one electron which moved through an electric potential difference of $1 \mathrm{~V}$ (Volt); $1 \mathrm{eV}=1.6^{*} 10^{-19} \mathrm{~J}$

$\mu \mathrm{m} \quad$ micrometer $1 \mu \mathrm{m}=10^{-6} \mathrm{~m}$

$\mathrm{Hz} \quad$ Hertz a measure of frequency in time $\left(1 \mathrm{~Hz}=1 \mathrm{~s}^{-1}\right)$ 


\section{GLOSSARY}

\begin{tabular}{|c|c|}
\hline Asthma & $\begin{array}{l}\text { Asthma is a chronic inflammatory disease of the airways leading to persistent } \\
\text { or recurring symptoms like airway obstruction, bronchi wall thickening } \\
\text { causing impaired lung functionality. Asthma (besides feline asthma) is solely } \\
\text { found in humans. }\end{array}$ \\
\hline Beamline & $\begin{array}{l}\text { In Synchrotrons electrons are forced to circulate by altering the direction of } \\
\text { movement using so called bending magnets. Changing the direction of the } \\
\text { electrons causes them to emit radiation in line with their original path. The } \\
\text { energy of the radiation depends on the field strength of the used magnet and } \\
\text { is optimized for the chosen application. Each of these experimental setups } \\
\text { since they are organized in line of the emitted photon beam is called } \\
\text { beamline. }\end{array}$ \\
\hline $\begin{array}{l}\text { Complex Refractive } \\
\text { Index }\end{array}$ & $\begin{array}{l}\text { The complex refractive index combines both, phase shift }(\delta \text {, real part }) \text { and } \\
\text { attenuation }(\beta \text {, imaginary part). For x-ray's this index is usually denoted as: } \\
\mathrm{n}(\mathrm{r})=1-\delta+\mathrm{i} \beta \text {. }\end{array}$ \\
\hline $\begin{array}{l}\text { Contrast-to-Noise } \\
\text { ratio (CNR) }\end{array}$ & $\begin{array}{l}\text { The contrast-to-noise ratio (CNR) describes the probability to discriminate } \\
\text { between two adjacent tissues within a data set. This probability increases } \\
\text { with a larger difference of the average signal level (g1-g2, contrast) and is } \\
\text { decreased by the noise level (usually measured as the standard deviation } \\
\text { (std) in a region assumed to be homogenous). } \\
\text { CNR }=\left(g_{1}-g_{2}\right) /\left(0.5^{*}\left(\operatorname{std}_{1}^{2}+\operatorname{std}_{2}^{2}\right)\right)^{0.5}\end{array}$ \\
\hline Edge Index (EEI) & $\begin{array}{l}\text { One way to quantify the quality of an edge present in a data set is the } \\
\text { calculation of the edge index (EEI). In analogy to the CNR it compares the } \\
\text { difference of the minimum and maximum values (L, P) of a line profile } \\
\text { through an edge of two adjacent tissues with the "noise" meaning the } \\
\text { standard deviation (STD) of the line profiles in areas which should be inside } \\
\text { the homogenous regions of the two tissues. } \\
\text { EEI = (P-L) } /\left(\mathrm{std}_{\mathrm{L}}{ }^{2}+\mathrm{std}_{\mathrm{P}}{ }^{2}\right)^{0.5}\end{array}$ \\
\hline Eosinophil & $\begin{array}{l}\text { Eosinophil granulocytes are white blood cells which are responsible to tackle } \\
\text { multicellular parasites and some infections. They are known to play an } \\
\text { important role in eosinophilic allergy and asthma. }\end{array}$ \\
\hline $\begin{array}{l}\text { Figure of Merit } \\
\text { (FoM) }\end{array}$ & $\begin{array}{l}\text { A Figure of Merit (FoM) represents a parameter which can be quantified and } \\
\text { used for comparison for instance between different algorithms. }\end{array}$ \\
\hline $\begin{array}{l}\text { Free propagation } \\
\text { phase contrast CT }\end{array}$ & $\begin{array}{l}\text { Free propagation phase contrast CT is one phase sensitive CT technique, } \\
\text { which in addition to the x-ray attenuation related contrast of classical CT } \\
\text { shows edge enhancement effects related to interferences of wave fronts } \\
\text { traveling with different velocities in adjacent materials. The magnitude of } \\
\text { these effects depends on the distance of the detector plane to the object plane. } \\
\text { At least partial coherence of the used x-ray's is the prerequisite for phase }\end{array}$ \\
\hline
\end{tabular}




\begin{tabular}{|c|c|}
\hline & $\begin{array}{l}\text { contrast CT. Therefore, it is restricted to Synchrotron Light Sources or micro } \\
\text { focus x-ray tubes. Besides free propagation other phase sensitive x-ray } \\
\text { techniques such as interferometric or edge-illumination methods exist. }\end{array}$ \\
\hline Holotomography & $\begin{array}{l}\text { Tomographic reconstructions of the 3D distribution of the refractive index or } \\
\text { "Holotomography" is implemented by rotating the sample and recording at } \\
\text { each projection angle a series of images at different distances. }\end{array}$ \\
\hline Macrophage & $\begin{array}{l}\text { The macrophage is a white blood cell belonging to the non-specific innate } \\
\text { immune system. It can perform phagocytosis and therefore can engulf larger } \\
\text { particles, which makes it especially attractive as carrier for diagnostic as well } \\
\text { as therapeutic substances. Depending on its differentiation it can either cause } \\
\text { inflammatory or anti-inflammatory effects. }\end{array}$ \\
\hline $\begin{array}{l}\text { Ovalbumin induced } \\
\text { asthma mouse } \\
\text { models }\end{array}$ & $\begin{array}{l}\text { Ovalbumin (OVA) is a protein in egg white, which is often used in research to } \\
\text { induce an allergic reaction in mice, by mimicking certain aspects of human } \\
\text { asthma. Usually, these models contain two or more immunization steps to } \\
\text { achieve an immune response and several challenging steps to cause the acute } \\
\text { allergic reaction. Severity of asthma symptoms in mice depends on the time } \\
\text { schedule and the amount of OVA applied in each step. }\end{array}$ \\
\hline Phase retrieval & $\begin{array}{l}\text { Phase retrieval is the process to calculate the real part of the refractive index } \\
\text { present in the analyzed sample. Several different algorithms are known either } \\
\text { using data sets with several sample-to-detector distances or a combination of } \\
\text { a priori knowledge of the sample in combination with only one sample-to- } \\
\text { detector distance data set. }\end{array}$ \\
\hline Plethysmography & $\begin{array}{l}\text { Plethysmography is a technique to measure changes in volume within an } \\
\text { organ or whole body (usually resulting from fluctuations in the amount of } \\
\text { blood or air it contains). }\end{array}$ \\
\hline Segmentation & $\begin{array}{l}\text { Segmentation is the process to separate the structure of interest from the } \\
\text { background in a data set, which usually leads to a binary decision (mask). }\end{array}$ \\
\hline
\end{tabular}




\section{INTRODUCTION}

\section{BACKGROUND}

Asthma is a global burden of public health predominately in industrial nations and continues to be a major cause of morbidity [1]. Tim Clark, Chair of the Global Initiative against asthma (GINA) pointed out that: "It is now estimated that as many as 300 million people of all ages, and all ethnic backgrounds, suffer from asthma and the burden of this disease to governments, health care systems, families, and patients is increasing worldwide" (Figure 1).

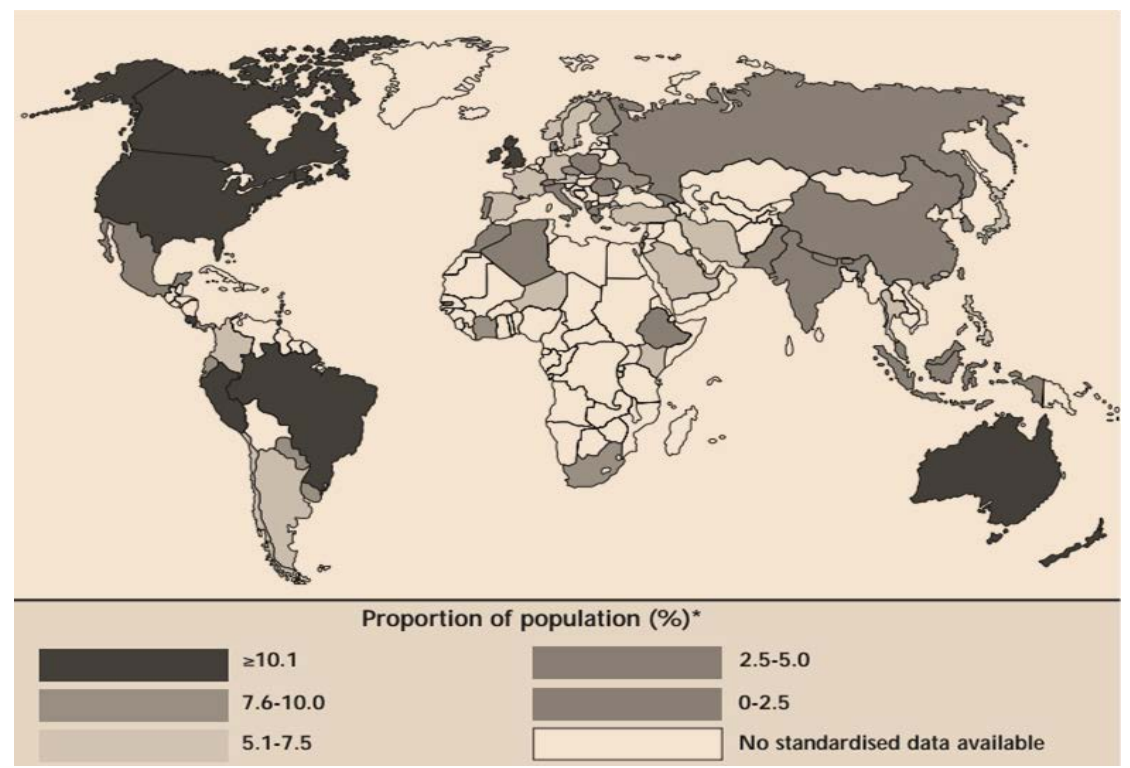

Figure 1 World map of prevalence of clinical asthma [2].

However, not all of the pathological mechanisms in asthma are fully understood and the commonly applied therapies are far from being optimal and can cause significant side effects [3]. Despite the fact that a large proportion of patients with asthma can be treated very effectively with corticosteroids, some patients do not respond to such a therapy at all [4]. Asthma is a disease which involves virtually all parts of the immune systems, a system so complex that up to now it cannot be reproduced ex vivo or studied by computer models. Therefore, animal models or more precisely allergic airway disease mouse models are frequently used in preclinical asthma research, although asthma can only be found in humans and felines. The predictive value of mouse models is controversially discussed [5]-[8] although all authors concluded that no model exists which can reliably mimic human asthma for the entire course of the disease. Even human asthma developes with a wide range of variations. Nevertheless, it is proven that depending on the mouse model, certain aspects of human asthma like eosinophilia, airway hyper-responsiveness or different grades of severity can be mimicked. Thus, despite the known limitations, preclinical allergic airway disease mouse models are still very important. In our studies we focus on ovalbumin induced asthma mouse models, because they are extensively studied in the literature, can be produced both easily and reliably and unlike for instance house dust mite models pose no risk for the examiner. 
Asthma is a very complex multi-factorial disease resulting in alterations within the lung on the anatomical, functional and molecular level. Figure 2 shows which of these hallmarks of asthma can potentially be exploited for imaging. On a cellular and molecular level, imaging approaches may focus on direct visualization of specific cells involved in the asthmatic reaction, by following their migration or by depicting changes in the expression level of specific enzymes, such as proteases. If anatomical imaging with a sufficient spatial resolution to resolve the lung substructure can be achieved, known effects like thickening of bronchial walls, obstruction of the airways as well as changes in the composition of the lung soft tissue present interesting endpoints that could be monitored. Even on a larger scale, changes in the total lung volume, in lung water content (due to inflammation or edema) and an adaption of the total lung structure to the alteration in its substructure are expected and represent interesting parameters to be visualized by in vivo anatomical imaging. Finally, from a more clinical point of view functional parameters such as quantification of lung function, "air-trapping" or the underlying changes in lung tissue elasticity, which directly reflect clinical symptoms, are of significant interest. All these imaging techniques and strategies are different and have their own requirements, advantages and limitations.

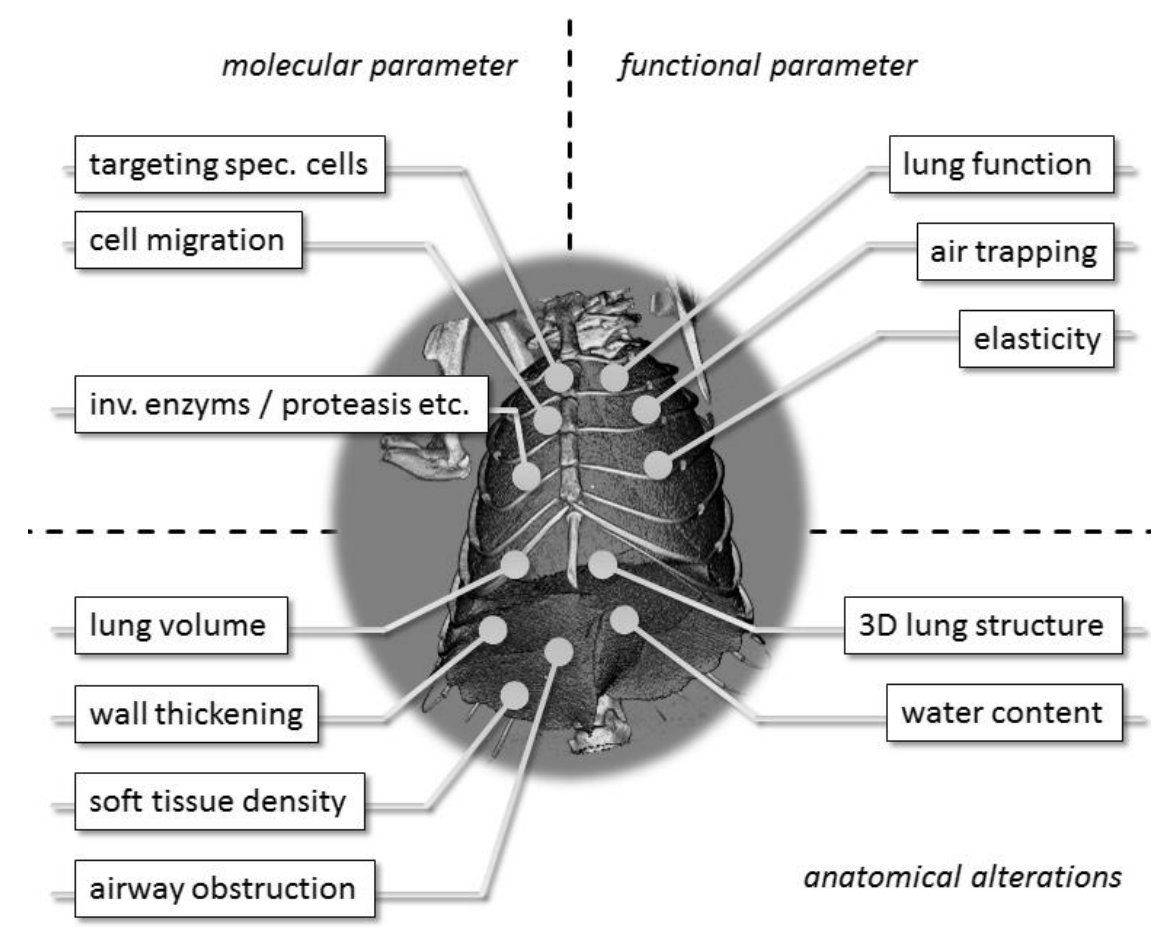

Figure 2 Overview of asthma hallmarks which presents potential targets for imaging distributed in molecular, functional and anatomical parameter.

\section{ANATOMICAL IMAGING}

The use of imaging techniques would be highly desirable in mice to allow the assessment of small morphological alterations and thereby to study pathological mechanism within allergic airway inflammation mouse models as well as to monitor the course of the disease and the efficacy of novel therapies. However, most of the techniques applied so far are hampered simply be the smallness of the mouse as a model organism and by the highly porous nature of the lung structure itself, which 
causes strong scattering in optical imaging and susceptibility artifacts in MRI. In addition, medical ultrasound is barely usable due to the echoes caused by the ribcage. Regarding in vivo imaging, all techniques suffer from motion artifacts caused by the movements of the chest during breathing. Due to the small size of the mouse lung a high spatial resolution in the order of $<10 \mu \mathrm{m}$ is needed to depict the lung substructure down to alveolar level. Moreover, the shape of the lung is critically dependent on its boundary condition represented by the rib cage and the diaphragm. Therefore, if in vivo imaging is not feasible, reliable structural parameters can only be assessed in an in-situ setup. Up to date microCT is the only 3D imaging method which can image entire mouse lungs in-situ with a sufficient spatial resolution to analyze their fine structure. An in vivo application of this method, however, is challenging due to the fact that the image quality (mainly the influence of noise) is intrinsically linked to the inverse spatial resolution to the power of 2 and to the inverse square root of the applied x-ray dose [9]. Ford et al. analyzed the relation of image quality and dose for an idealized CT imaging system [9]. He measured a dose of 0.35Gy for a mouse imaged with an isotropic resolution of $135 \mu \mathrm{m}$ assuming a $1 \%$ coefficient of variation (COV) of the linear attenuation coefficient. Therefore, imaging with the desired resolution of $10 \mu \mathrm{m}$ and the same noise level would yield to a dose of $\sim 64 \mathrm{~Gy}\left(0.35 \mathrm{~Gy}^{*}(135 / 10)^{\wedge} 2\right)$. This is of course an enormously high entrance dose, but underlines that in vivo imaging cannot be performed with a sufficient spatial resolution.

Classical x-ray attenuation based microCT of specimens is usually characterized by a high spatial resolution, but expresses a poor soft tissue contrast and a high noise level. Nevertheless, it has been already successfully applied in mouse lung disease models usually in combination with a preparation scheme to fix and explant the lung tissue as for instance described by Vasilescu et al. [10]. Figure 3 shows exemplary cross sections of in-situ mouse lung scans for a healthy mouse, an asthmatic mouse and a mouse from a lung emphysema model obtained with a classical bench-top microCT (eXplore Locus SP, GE HealthCare). The images appear grainy due to the high noise level, which substantially hampers a detailed analysis of the lung substructure. The noise problem can be overcome by extended scanning times in the range of hours, providing a better signal-to-noise ratio. However, this requires that the sample remains stable for such a long period. In summary, all these issues do not only limit a microCT based assessment of the lung anatomy at $10 \mu \mathrm{m}$ resolution to an in-situ application, but also underline that a meaningful image quality can only be reached with long scanning times putting high demands on sample quality and preparation. 


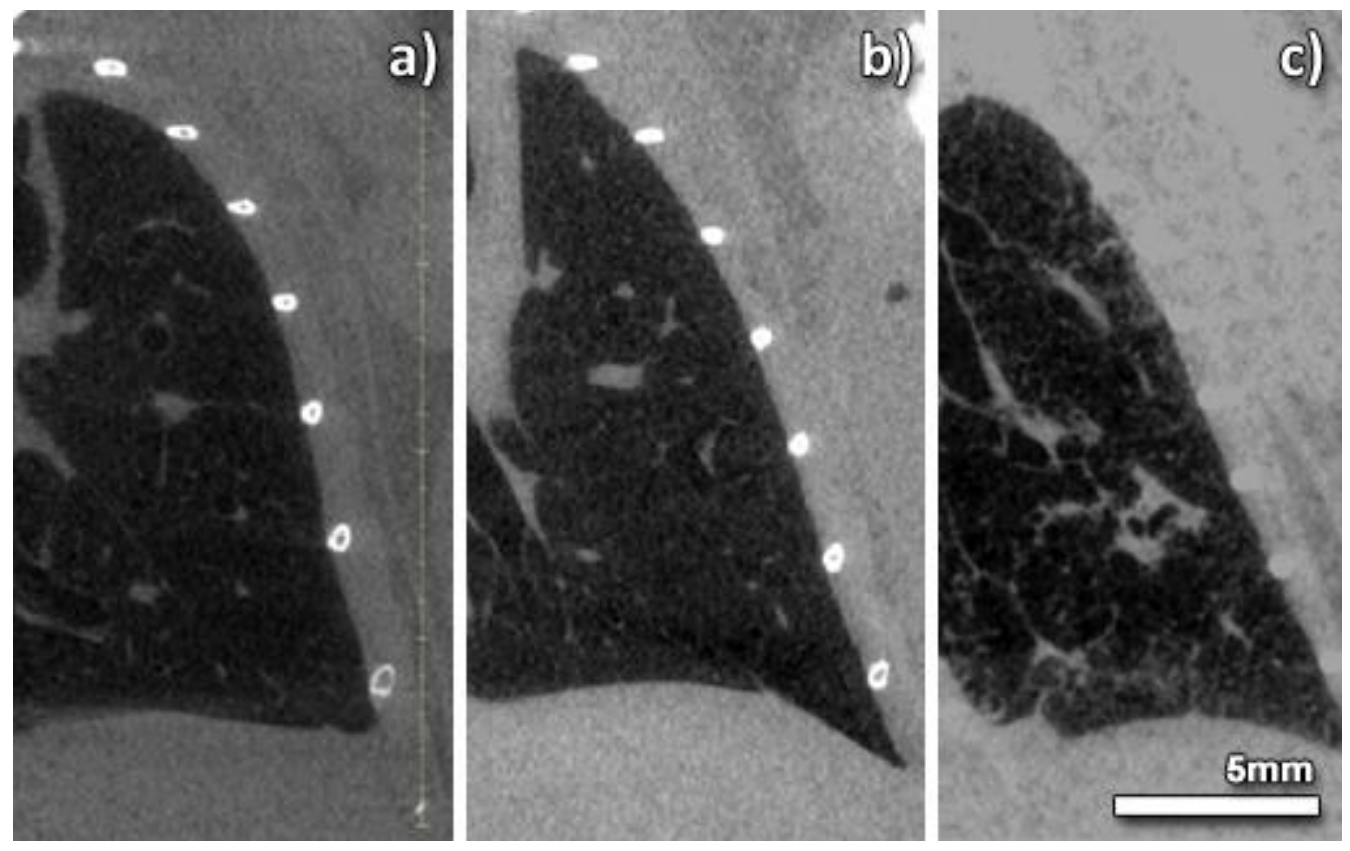

Figure 3 Cross sections through lung scans acquired with eXplore locus SP microCT demonstrate a high noise level: In-situ mouse lungs inflated with air at a pressure of $30 \mathrm{~cm}$ water column of a) healthy mouse and mice with b) asthma and c) emphysema. Due to the high noise level the lung substructure cannot be resolved in detail. Therefore besides the obstruction of big bronchi, no structural differences between wild type controls and asthmatic mice were found. Scanning time 1.5h, Resolution $\sim 16 \mu \mathrm{m}$

Absorption is not the only interaction of x-rays with matter. In addition, scattering (however with very low scattering angles) and phase shift occur (Figure 4). The contribution of these effects to the measured image contrast can only be seen if at least partial coherent x-rays are used. Therefore, the phase effect, though well known for visible light in classical optic, was first discovered for x-rays at a Synchrotron Light Source and its application for biomedical samples was pioneered as late as 1995 by Prof. Atsushi Momose et al. [11]. Phase contrast occurs when partial coherent x-ray waves which traveled through different tissues at slightly different velocity overlap and interfere. For the simplest setup (in-line free propagation phase contrast) no additional optical elements are needed. In this setup, the detector is placed in a certain distance behind the sample. The magnitude of the interference depends on the plane or more precisely the sample-to-detector distance at which the image is recorded. As a result absorption and fringes caused by the phase shift of the waves are combined on the measured projection image. As a special case in the near contact regime (sampleto-detector distance is very low) the image resembles the classical absorption based contrast. The outcome in the near-field distance is an image with remarkably enhanced edges even for low absorbing materials. In contrast to all other imaging techniques that can hardly deal with lungs, lung imaging is an ideal application for phase contrast CT [12], [13]. However, the mentioned edge effects that strongly enhance the delineation of low-absorbing objects, renders quantitative analysis of the samples challenging, if simple threshold based segmentation is used. In order to decouple absorption from phase effects calculating images predominately displaying the real part of the complex refractive index (responsible for the phase shift) a special type of algorithms can be applied. It was demonstrated by Beltran et al. [14] that the combination of inline free propagation phase contrast CT with the application of phase retrieval algorithms produces images that 
demonstrate an up to 200 fold gain in contrast-to-noise ratio (CNR) for soft tissue. Therefore this technique has been chosen as the central method in this thesis.

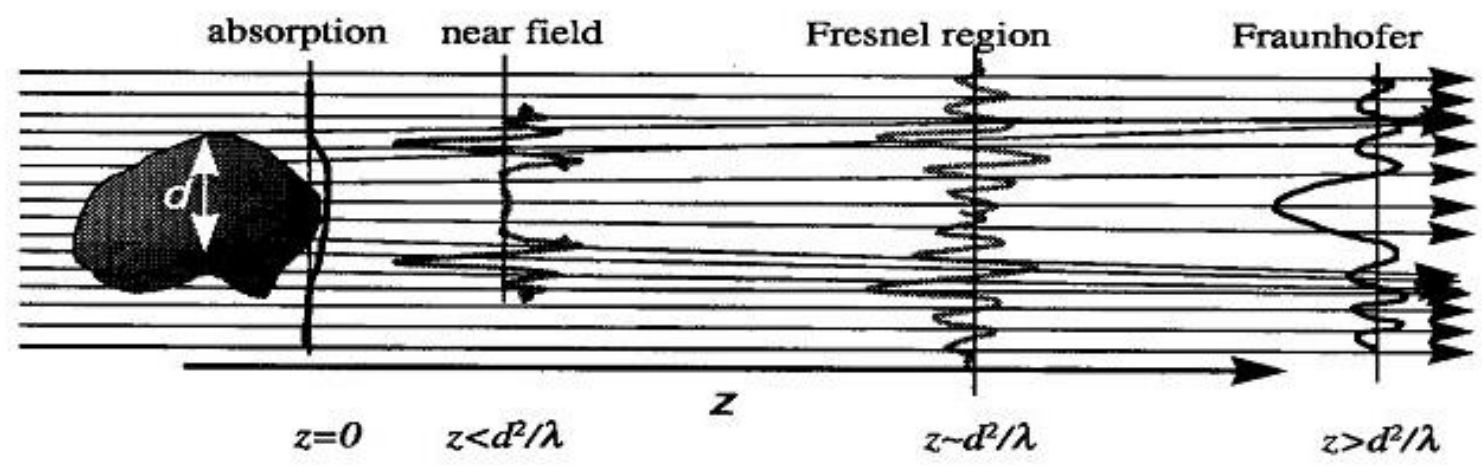

Figure 4 Impact of the phase shift on the image formation with increasing sample-to-detector distance:

This figure demonstrates how the phase shift of the incident $x$-ray beam that occurs within the sample causes different interference effects depending on the distance between the imaging plane and the object (Bronnikov et al. [15]). In this thesis all phase contrast data sets have been recorded in the near field regime.

Despite the fact that free propagation phase contrast CT imaging has already been used for lung imaging, some specific problems remained for its application in in-situ lung imaging. Therefore the aims of this thesis were:

- to use single distance phase retrieval since it needs only projection images acquired at one single sample-to-detector distance and therefore it would be the fastest approach less influenced by alterations of the sample over time. However, problematic in this perspective may be that single distance phase retrieval algorithms need some a-priori information about the object, which may not be valid in in-situ lung imaging. Therefore, the applicability of single distance phase retrieval algorithms for in-situ mouse lung imaging needs to be evaluated.

- to validate if the image quality and resolution is sufficient to detect and quantify the subtle alterations of the lung structure especially in mild asthma models.

- to verify if the gain in image contrast provided by phase contrast CT in combination with single distance phase retrieval will be sufficient to track labeled macrophages within mouse lungs in-situ.

\section{MEASUREMENT OF FUNCTIONAL PARAMETERS}

Anatomical and molecular or cellular alterations in asthmatic lungs present interesting hallmarks of the disease, but if and to what extent these features correlate to clinical symptoms, in particular to an impairment of the lung function, is not evident. In order to address this problem, direct assessment of lung function would be of utmost importance. However, while easily feasible in humans, such an approach has some intrinsic problems in mouse models. Measurement of the lung function in mice is commonly done by plethysmography [16]. This technique usually either needs intubation or at least restraining of the mouse, both introducing a high level of stress. As asthma is known to be triggered by stress in humans, stress caused during plethysmography may interfere 
with the results. Plethysmography with unrestrained mice has been reported but is controversially discussed in the literature. Whereas Adler et al. demonstrated that this method leads to unreliable measurements of airway hyper responsiveness [16], deLorme and Moss showed that in contrast to the traditional methods unrestrained plethysmography was able to identify airway reactivity [17]. However, the analysis of alterations in lung function in addition to other readouts used in asthma research seems to be of critical importance, as it presents the only direct link to the clinical symptoms and should be the most important criteria to preclinical evaluate novel asthma therapies in mouse models.

Therefore, the question aimed to be answered within this thesis is:

- Can an alternative reliable imaging strategy, less stressful than plethysmography, be developed to directly measure lung function in living mice?

\section{MOLECULAR IMAGING}

In vivo molecular imaging aims to depict molecular processes within the entire living subject. To achieve this goal usually targeted probes are administered. Since only a limited amount of such a probe can be concentrated at the sites of interest, imaging techniques such as PET, SPECT and Optical Imaging (either by near infrared fluorescence (NIRF) or bioluminescence) must deal with a low amount of photons and therefore require a very high sensitivity. To allow enough photons to interact with a detector element to produce a measurable signal, the detectors used need to have a sufficient large pixel size, which substantially limits the spatial resolution. In my thesis I focused on optical imaging, in particular NIRF imaging [18]. This is a method that is extensively used in preclinical research today, as in contrast to PET or SPECT, no radioactive probes are used and thus no specific radiation safety laboratories are needed. In addition, optical probes do not decay like the radioactive ones and are therefore stable for a long time and much cheaper. Usually the optical window of water and hemoglobin (in the range of $600-800 \mathrm{~nm}$ ) is exploited for imaging, allowing a penetration depth in the range of 2 to $4 \mathrm{~cm}$. This limitation has basically no implication for mice but substantially hampers clinical use of optical imaging and restricts it to superficial applications, such as the skin.

NIRF imaging has no known side-effects and therefore enables longitudinal in vivo studies. This in combination with the availability of dedicated fluorescence probes, has been the foundation for the successful use of this imaging strategy. The probes can be divided into four classes (Figure 5): a) targeted probes, combining for instance an antibody with a fluorescence dye to specifically highlight tumor cells; b) smart probes, which are activated upon a change in the environment; for example a quenched probe which becomes dequenched in the presence of an enzyme such as cathepsin during inflammatory processes; c) unspecific fluorescence dyes which can for instance be used to highlight the difference in the blood supply of certain regions within the body and d) in vitro cell membrane staining to allow tracking of injected labeled cells in vivo. 


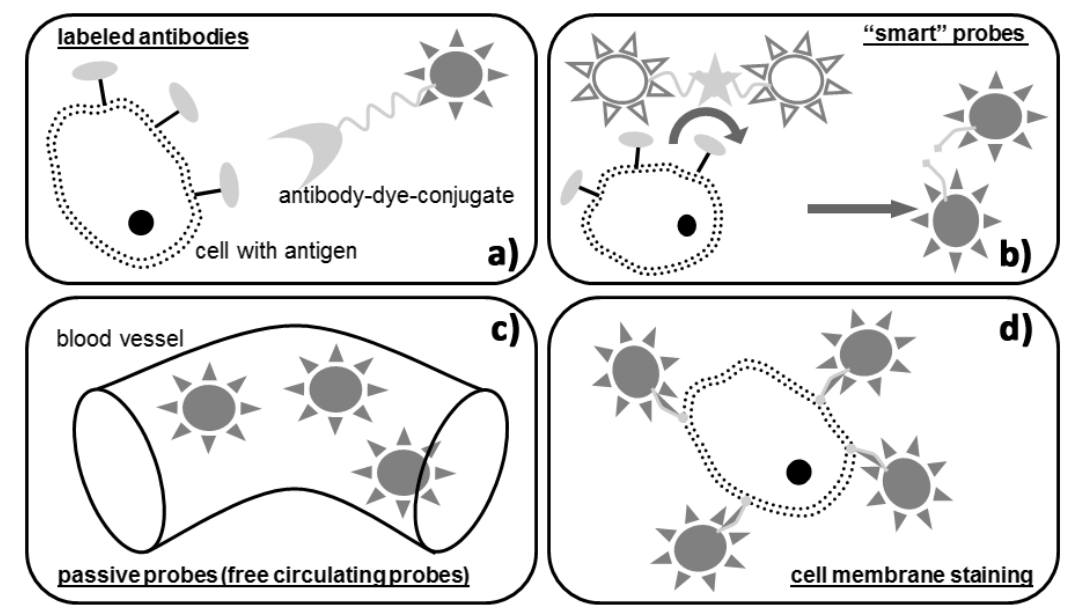

Figure 5 The four major probe concepts in NIRF Imaging;

a) a labelled antibody, b) a probe which is non-fluorescent in its ground state and gets activated by for instance specific enzymes, c) nonspecific probes which circulate in the blood stream, d) or cells which have been stained with a fluorescent dye.

In the past years various optical imaging applications have been reported in asthma mouse models, mainly focusing on the inflammatory aspect of the disease and the application of smart probes, which are activated in the presence of inflammation [19], [20]. This approach, however, has two intrinsic flaws: firstly, smart probes usually shows a slow kinetic due to the fact that a sufficient amount of the probe needs to be activated to produce a signal strong enough and secondly, the probes do not attach to the site of action/inflammation and therefore create a strong unspecific background signal, especially in the liver, which is the common excretion pathway of most of the molecules. In addition to the smart probes, the use of blood pool agents has been reported in an LPS induced lung inflammation mouse model allowing the depiction of the changes in the lung blood content in presence of inflammation [20]. Neither antibody based nor cell tracking studies have been reported for optical imaging in asthma mouse models so far.

In order to improve the applicability of NIRF imaging in asthma mouse models, within this thesis, imaging strategies were developed:

- to establish an readout faster than the $24 \mathrm{hrs}$ as required by the smart-probes. This would be required to monitor the acute asthma attack and a treatment response, since measurements in humans show that there is a drop in lung function $4 \mathrm{~h}$ after an acute asthma attack. After this time the lung function starts to normalize again only to show a second minor reduction in a late phase.

- to specifically image immune cells, since the recruitment of these cells such as eosinophils and macrophages into the lung plays an important role in asthma. 


\section{SUMMARY OF THE AIMS OF THESIS}

In summary the thesis aims to develop an imaging platform to study certain morphological, functional and molecular aspects of asthma in preclinical allergic airway disease mouse models. This platform will provide tools to further analyze the patho-mechanism of the disease as well as to better assess effects of new treatments targeting hallmarks of asthma.

\section{RESUlTS \& DisCUSSION / SUMMARY OF PUBLICATIONS}

The results will be presented grouped into anatomical, functional and molecular imaging. Therefore, the discussed publications will not appear in chronological order.

\section{A. AnAtomical Imaging}

\section{THE SAMPLE PREPARATION SCHEME}

The aim of applying anatomical imaging in this thesis is the quantification of structural alterations within the lung of asthmatic mice. Unlike for instance emphysema, which can cause major and therefore easily detectable damage to the lung parenchyma, asthma produces only mild alterations of the lung tissue. In addition, it is desirable to be able to distinguish between severe chronic and mild acute asthma as well as to quantify treatment response. In order to achieve a spatial resolution of $10 \mu \mathrm{m}$, an increased x-ray dose is required which is too high for in vivo imaging, thus restricting our technique to be used only in dead animals. In addition, to improve image quality by increasing the CNR, long scanning times in the range of hours are needed during which the sample must not alter or move. Furthermore, the lungs in mice collapse soon after death. Therefore, it is crucial that a sample preparation will be employed which mimics the physiological situation of the lung as close as possible, as well as suppresses both, deformation and alteration of the sample. To this end we developed a scheme to prepare in-situ mouse lung samples. First, we performed an ex-vivo tracheotomy, inserted a cannula into the trachea and inflated the lung with air at a constant pressure of $30 \mathrm{~cm}$ water column. Following this procedure the entire mouse was embedded in a plastic tube using 1\% agarose gel as described by Dullin et al. [21]. The agarose gel sufficiently suppresses sample movements and shows very little x-ray attenuation. Adversely, the gel cracks at temperatures below $0^{\circ} \mathrm{C}$ and the prepared samples can therefore not be stored frozen. Despite the improved soft tissue contrast in in-line phase contrast CT used in this study, soft tissue can poorly be discriminated from liquids. Fixatives like ethanol or formalin cannot be applied since they would interfere with the status of the swollen airway walls and therefore, samples cannot be maintained for a long time. Moreover, a stable temperature needs to be provided to avoid severe motion artifacts created by thermal expansion of the air inside the lung. Based on these requirements we improved the sample preparation scheme by adding a $30 \mathrm{~min}$ time delay between inflating the lung and embedding the mouse, to let the corpse relapse from rigor-mortis. As the samples are completely sealed in agarose gel within the plastic tube they can be maintained at $4^{\circ} \mathrm{C}$ for about one week. About 30mins before imaging we removed the samples from the fridge and placed them inside the experimental hutch of the beamline to let them adjust to ambient temperature. This sample preparation scheme has proven to provide reliable and stable conditions for detailed in-situ lung imaging and was therefore chosen for all Synchrotron experiments presented here. 


\section{SINGLE DISTANCE PHASE RETRIEVAL - THE METHOD OF CHOICE FOR IN-SITU LUNG IMAGING}

In order to increase the soft tissue contrast within the lung we applied Synchrotron free propagation in-line phase contrast. The resulting images show strong edge enhancement, which certainly improves visibility of tissue interfaces but hampers the segmentation of the different compartments within the lungs and therefore prohibits quantification. In order to circumvent this situation we applied phase retrieval to decouple phase from absorption based contrast. Most of these classes of algorithms assume that the absorption based contrast is nearly independent from the sample-to-detector distance, whereas the phase effect shows a strong dependency on distance. As a result, projection images acquired at two or more sample-to-detector distances are commonly analyzed. This however is disadvantageous in our case, not only because changing the distances introduces alignment problems of the obtained projection images but more importantly because it prolongs the scanning times and thereby promotes potential alterations in the unfixed tissues. For this reason we focused on the single distance phase retrieval method, which only requires projection data obtained at one distance, but in addition needs a priori information of the sample. This priori information contains the assumption of homogenous objects, meaning an object which either shows only absorption (real part of the refractive index $=0$ ), only phase shift (imaginary part of the refractive index $=0$ ) or a constant ratio between the real and imaginary part of the refractive index. These idealized conditions are not met in in-situ mouse lung samples. Hence, the applicability, reliability and benefit of single distance phase retrieval for the multi-material biological samples used here needed to be evaluated.

\section{1) ACCURACY AND PRECISION OF RECONSTRUCTION OF COMPLEX REFRACTIVE INDEX IN NEAR-FIELD SINGLE-DISTANCE PROPAGATION-BASED PHASE-CONTRAST TOMOGRAPHY}

T. Gureyev, S. Mohammadi, Y. Nesterets, C. Dullin, and G. Tromba, "Accuracy and precision of reconstruction of complex refractive index in near-field single-distance propagation-based phase-contrast tomography," J. Appl. Phys., vol. 114, no. 14, p. 144906, 2013.

The aim of using phase retrieval is to decouple phase from absorption information and in doing so to allow the reconstruction of both the real and imaginary part of the refractive index $(1-\delta-i \beta)$. Due to the fact that single distance phase retrieval assumes a homogenous object the aim of this part of the study was to test how precise the refractive index can be recovered in samples containing a mixture of different materials. Therefore, I designed a simple phantom made from acrylic glass with 6 cylindrical holes filled with substances of known chemical composition related to applications in biomedical imaging: polyoxymethylene $\left(\mathrm{CH}_{2} \mathrm{OH}\right)$, water $\left(\mathrm{H}_{2} \mathrm{O}\right)$, air, Ultravist $300\left(\mathrm{C}_{18} \mathrm{H}_{24} \mathrm{I}_{3} \mathrm{~N}_{3} \mathrm{O}_{8}\right.$, a clinically used contrast agent), calcium chloride $\left(\mathrm{CaCl}_{2}\right)$, magnesium chloride $\left(\mathrm{MgCl}_{2}\right)$ and glycerol $\left(\mathrm{C}_{3} \mathrm{H}_{8} \mathrm{O}_{3}\right)$. The advantage of using such a phantom with defined materials was that the theoretical $\beta$ and $\delta$ could be calculated. We demonstrate that, except for regions near the interfaces between distinct materials, the distribution of the imaginary part ( $\beta$, responsible for absorption) of the refractive index, can be accurately reconstructed from a single projection image per view angle using single distance phase retrieval in combination with conventional CT reconstruction. In contrast, the accuracy of reconstruction of the imaginary part ( $\delta$, responsible for the phase shift) depends strongly on the choice of the "regularization" parameter within the algorithm. We demonstrate that for some multi-material samples, a direct application of this method produces qualitatively incorrect results for $\delta$, but which can be rectified by utilizing suitable a priori 
information about the sample. Especially, if materials with a large difference in their respective $\delta-\tau \mathrm{o}-\beta$ ratio are present in the sample, the algorithm can only adequately calculate one of the materials at a time. Respectively, for in-situ lung imaging in a cross-section of the mouse chest either the bones or the lung will be accurate. This finding has been further analyzed by Beltran et al. [22] who proposed the combination of reconstructed data sets optimized for each material of interest. Additionally, in our study we found that it is possible to significantly improve the CNR by increasing the sample-to-detector distance in combination with the application of phase retrieval compared to conventional ("contact") CT.

2) QUANTITATIVE EVALUATION OF A SINGLE-DISTANCE PHASE-RETRIEVAL METHOD APPLIED ON IN-LINE PHASE-CONTRAST IMAGES OF A MOUSE LUNG

S. Mohammadi, E. Larsson, F. Alves, G. Tromba, and C. Dullin, "Quantitative evaluation of a single-distance phaseretrieval method applied on in-line phase-contrast images of a mouse lung," J. Synchrotron Radiat., vol. 21, no. 4, pp. 0-0, 2014.

In a second step we evaluated if the knowledge we gained from the phantom study can be applied in in-situ imaging of mouse lungs. We performed inline free propagation phase contrast CT of an in-situ mouse lung sample at three different sample-to-detector distances $(7,30$ and $100 \mathrm{~cm})$. As expected the reconstructed raw data sets showed an edge enhancement that progress with increasing detector distance. We were able to verify that the application of single distance phase retrieval in combination with 3D reconstruction in all three cases produces comparable data sets predominately showing the $\delta$-values of the refractive index. In order to assess the image quality the CNR was measured. A 10 fold gain in CNR was observed between the phase retrieved data set at $30 \mathrm{~cm}$ compared to the reconstruction of the raw projection data at $7 \mathrm{~cm}$ mimicking classical absorption based CT. It has to be pointed out that since the contact regime data was acquired with a quasi-monochromatic x-ray source, comparison of the phase retrieved data sets with classical microCT utilizing a fine focus X-ray tube would have revealed an even higher gain in CNR. However, we also demonstrate that CNR as a single measure of image quality is insufficient as it does not consider the sharpness of an image. Since established measures of image sharpness are unable to compare the images with strong edge enhancement (reconstructed unprocessed data sets) with the phase retrieved data sets, we introduced a new measure of image sharpness based on a non-linear fit of a sigmoid function into edge-brightness profiles.

By applying this measure we found that the 10 fold gain in CNR is, as expected, related to a reduced edge sharpness, which is in accordance with the visual observation of the data sets, but provides a reliable edge profile without phase shift-related edge effects. These results are in accordance with the findings of $\mathrm{Wu}$ et al. [23] who demonstrated that even by violating the "homogenous object" assumption the obtained data is correct for tissue containing only materials with an atomic number lower than 11, such as in the mouse lung. Our observations (demonstrated in Figure 6) proved that we can increase the contrast by simultaneously maintain sharp edges in the image and substantially suppress the edge effects in the raw data sets. As a result, in these phase retrieved data sets air and soft tissue are represented by two clearly distinguished grey value intervals allowing their segmentation using a simple threshold function. Thus, the results of this experiment proves that 
analysis of in-situ mouse lung samples will be feasible and a more than 10 fold improved sensitivity can be expected.

In theory and in accordance with our first study, the CNR can further be improved by increasing the sample-to-detector distance even more. However, we found a small decrease in the edge quality from $30 \mathrm{~cm}$ to $100 \mathrm{~cm}$ detector distance which may be related to an increased effect from scattered photons. Therefore, a detector distance of $30 \mathrm{~cm}$ was chosen for the following studies.

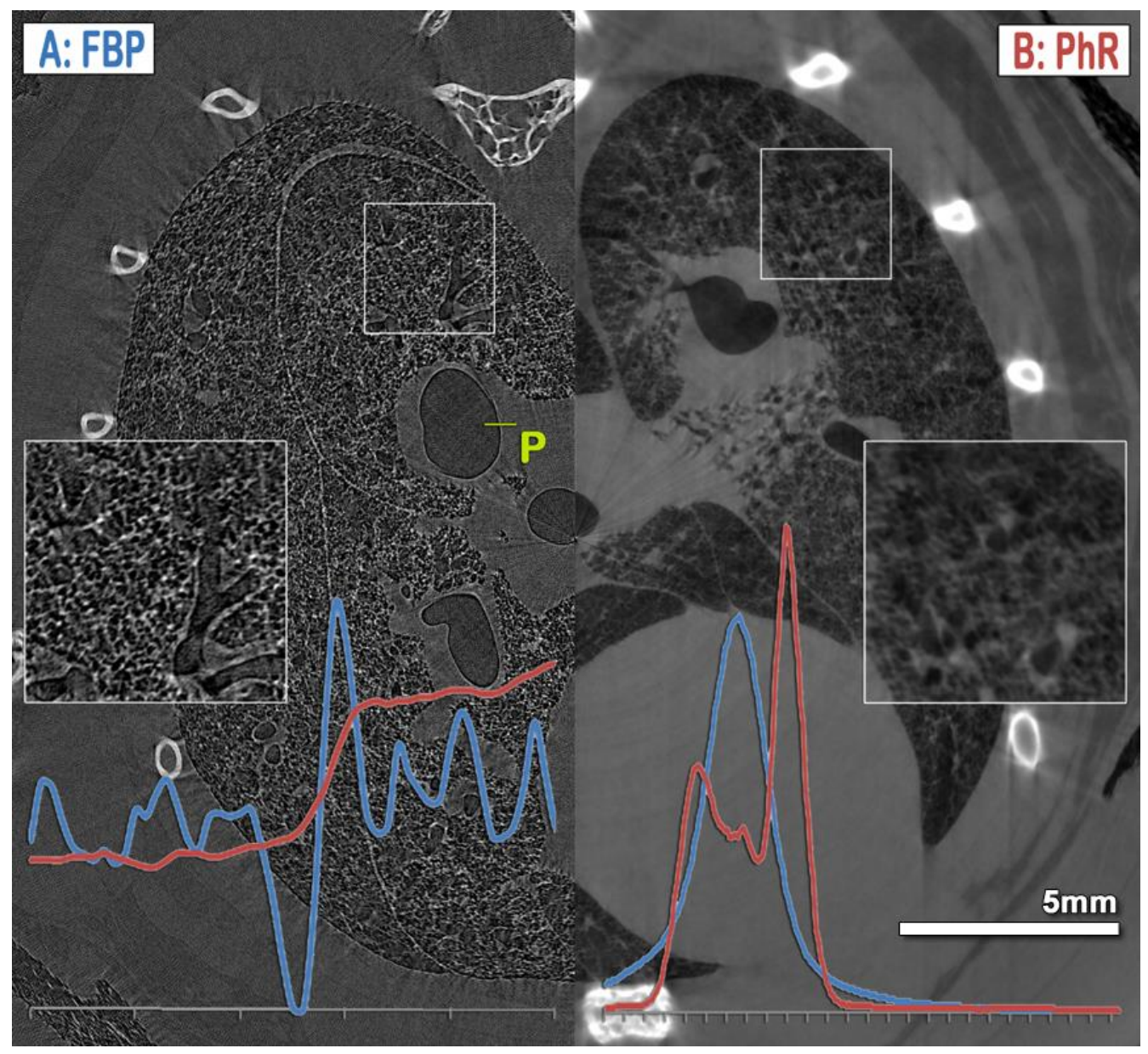

Figure 6 The benefits of phase retrieval in phase contrast Lung CT:

An exemplary slice of an in-situ mouse lung scanned at 30cm sample-to-detector distance either reconstructed directly (A: FBP) or after application of single distance phase retrieval (B: PhR). The profile plot along P (detail view left panel) show strong edge effects for FBP. These effects produce all kinds of grey values resulting in a grey value histogram of the lung area basically showing one big gaussian function (right insert, blue curve). The grey value histogram of the same area in the phase retrieved data clearly demonstrates two components (air and soft tissue, red curve). In addition the profile plots at the position (P) (left insert), show that by application of single distance phase retrieval the edge effects are successfully removed and the interface between air and soft tissue resembles the expected simple stair shape. 


\section{3) PHASE CONTRAST CT FOR QUANTIFICATION OF STRUCTURAL CHANGES IN LUNGS OF ASTHMA MOUSE MODELS OF DIFFERENT SEVERITY}

C. Dullin, E. Larsson, G. Tromba, A. Markus, F. Alves, "Phase contrast CT for quantification of structural changes in lungs of asthma mouse models of different severity," J. Synchrotron Radiat. vol. 22, pp. 1106-1111, 2015

Anatomical hallmarks of asthma such as airway obstruction or airway wall thickening are reported in many publications, however little is known about the magnitude of these effects in asthma mouse models, especially in relation to the large varieties of established asthma models. Therefore, our aim was to test if the gain in CNR in in-line phase contrast CT can provide more insights into structural alterations of asthmatic mouse lung tissue. To this end we analyzed mice of two asthma models of different severity as well as healthy controls. We used ovalbumin (OVA) induced asthma mouse models, a very common and well established method. In order to generate asthma of different severity, different concentrations and amounts of OVA were applied (Figure 7).

a) mild allergic asthma model (MAA)

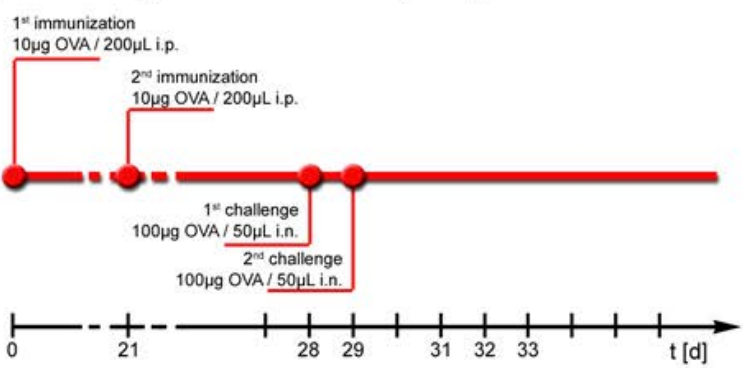

b) severe allergic asthma model (SAA)

$1=$ immunization

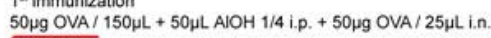

[20 immunization

50ug OVA/ $150 \mu \mathrm{LL}+50 \mathrm{HL}$ AIOH $1 / 4$ ip. $50 \mu \mathrm{g} O \mathrm{OVA} / 25 \mu \mathrm{L}$ i. . .

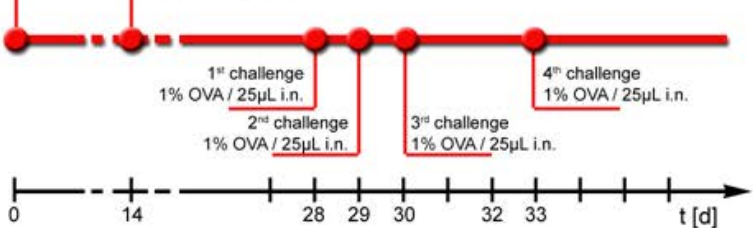

Figure 7 Schedule of the different OVA induced allergic airway inflammation mouse models used:

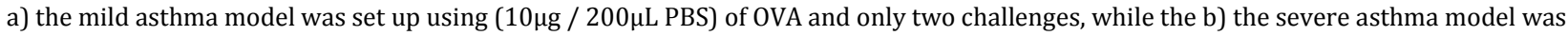

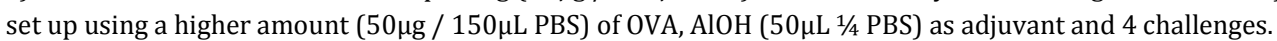

Twelve 6 week old female BALB/c mice were equally distributed into three different groups (healthy controls $(\mathrm{CN})$, mild allergic asthma (MAA) and severe allergic asthma (SAA)). The animals were sacrificed (in case of the asthmatic mice two days after the last challenge) and prepared for phase contrast analysis as described above. The time point was chosen based on pilot optical imaging studies that revealed a peak in the inflammatory response $48 \mathrm{~h}$ after the last OVA challenge. Phase contrast CT scans at the SYRMEP beamline (Synchrotron Light Source "Elettra", Trieste, Italy) were obtained at $22 \mathrm{keV}$ and with a sample-to-detector distance of $30 \mathrm{~cm}$. Single distance phase retrieval was performed and finally the data sets were reconstructed using a classical FBP algorithm. Figure 8 shows the quality of the obtained lung phase contrast CT data sets. 

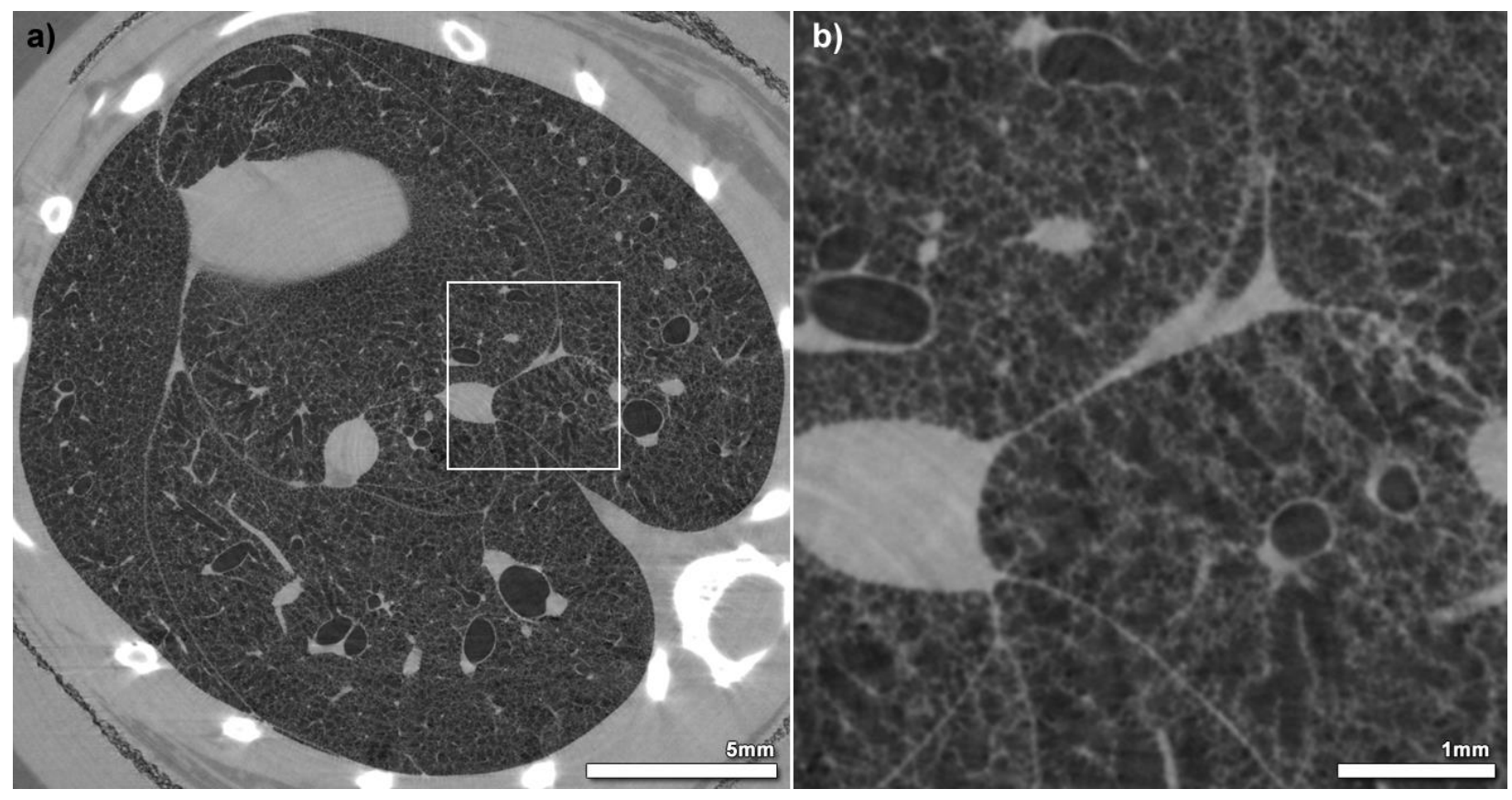

Figure 8 Demonstration of the image quality in inline phase contrast CT of in-situ lungs of a healthy mouse after phase retrieval: a) displays a representative slice of a phase retrieved and reconstructed data set at reduced resolution. Despite the good image quality within the lung the expected poor quality of the bones can be seen. b) shows a detailed view of the region indicated by the white rectangle in a) at full resolution. Given the fact that the alveolar septal tissue is virtually a single cell layer, the data show cellular resolution in 3D for the entire lung.

In these obtained data sets I analyzed the soft tissue volume ratio in 8 volumes of interest (VOI) with a size of $2 \times 2 \times 2 \mathrm{~mm}^{3}$ equally distributed in the periphery of the lung at comparable locations in each analyzed mouse. The peripheral lung region was chosen to avoid large bronchi inside the VOIs. The soft tissue volume ratio is the relative percentage of soft tissue within such a VOI, which is expected to be increased in the presence of airway wall thickening. I found significant differences in the soft tissue content between the three groups in correlation with the grade of severity of asthma (SAA shows a greater soft tissue volume ratio than MAA, while MAA shows a higher value than the controls). Moreover, the average $\delta$-value of the lung tissue also differed between healthy controls, mild and severe asthmatic mice. Interestingly, the mild asthma group showed a lower and the severe asthma group a higher value than the controls. Since the $\delta$-value is characteristic for the material, this indicated a possible difference in the composition of the lung tissue in these models. In order to verify this finding, lungs of mice from both models as well as from controls were explanted, weighed directly after excision and after being vacuum dried for $24 \mathrm{~h}$. Lungs from mice with severe asthma (SAA) were found to be twice as heavy as healthy controls, without showing any difference in wet and dry state, thus indicating that the ratio between the water content and the cells equals that in the healthy controls. In contrast, in the mild asthmatic mice (MAA) the weight difference of lungs increased from wet to dry state, thus indicating that the water to tissue ratio is higher in lungs of mild asthmatic than in healthy mice. This might indicate that in lungs of the MAA mice edema and mucus production is the more dominant effect rather than an increased amount of cells as found in the severe asthma model.

In summary, the results of these experiments proved that phase contrast CT at a spatial resolution of $9 \mu \mathrm{m}$ in combination with single distance phase retrieval is not only capable of quantifying 
structural differences in between lungs of healthy controls, mild and severe asthmatic mice, but is also sensitive enough to detect differences in the soft tissue composition in these asthma mouse models.

In a novel still unpublished study we repeated this experiment, but extended the three groups by a group of asthmatic mice treated with the common anti-inflammatory glucocorticosteroid Dexamethasone (DEX) as well as a group of mice sacrificed 5 days after the last OVA challenge. Both groups the treated mice and the mice left to recover on their own, displayed significantly lower soft tissue volume ratios than the respective untreated MAA and SAA mice, but still had higher softtissue volume ratios than the healthy controls. Even without treatment the mice of the MAA model showed values close to the baseline 5 days after the last OVA challenge, whereas the mice of the SAA model revealed a significantly larger soft tissue volume ratio than the healthy at the same time point. This indicates that in the severe model the alterations in the lungs are not reverted within 5 days. If these lung tissue changes in SAA indeed present persistent alterations will be the scope of further experiments.

In conclusion, the here established anatomical imaging procedure based on in-line free propagation phase contrast CT in combination with single distance phase retrieval appears to be well suited to assess morphological alterations in asthma mouse models of different severity as well as to address the effect of therapeutic strategies onto these morphologic features.

4) FUNCTIONALIZED SYNCHROTRON IN-LINE PHASE-CONTRAST COMPUTED TOMOGRAPHY: A NOVEL APPROACH FOR SIMULTANEOUS QUANTIFICATION OF STRUCTURAL ALTERATIONS AND LOCALIZATION OF BARIUM-LABELED ALVEOLAR MACROPHAGES WITHIN MOUSE LUNG SAMPLES

C. Dullint, E. Larssont, S. Dal Monegot, S. Mohammadi, M. Krenkel, C. Garrovo, S. Biffi, A. Lorenzon, A. Markus, J. Napp, T. Salditt, A. Accardo, F. Alves $\ddagger$, and G. Trombał, “Functionalized Synchrotron inline phase contrast CT: a novel approach for simultaneous quantification of structural alterations and localization of barium labelled alveolar macrophages within mouse lung samples," J. Synchrotron Radiat., vol. 22, pp. 143-155, 2015

$\dagger, \ddagger$ equal contribution

A drawback of CT is its limitation to structural imaging, since its rather poor sensitivity prohibits functional approaches. As demonstrated in the previous experiments our setup for in-line free propagation phase contrast CT in combination with single distance phase retrieval provides an at least 10 -fold increase in CNR, basically the same factor that separates classical CT from MRI in which functional imaging strategies exist. Therefore, I aimed to combine the superior spatial resolution of CT with a functional aspect to detect in-situ sites of inflammation. My approach established for the first time functional CT with targeted contrast for in-situ imaging of mouse lungs. For this purpose, we labeled MH-S cells (an immortalized alveolar macrophage mouse cell line [25]) with a clinically approved $\mathrm{BaSO}_{4}$ containing contrast agent (Micropaque [26]) and stained the membrane of these cells with DiD, a fluorescent dye [27]. Six million of these cells were intratracheally administered into the lungs of mice from the mild asthmatic model $48 \mathrm{~h}$ after the last challenge. Macrophages were chosen as vehicles for contrast agents for their phagocytotic 
properties, as well as for the facts that they are known to home to inflammatory sites and were recently identified as a key player in asthma [28]. Therefore, collecting more knowledge about the migration pattern of macrophages in asthma may also aid to a better understanding of the pathomechanism of this disease.

The location of the macrophages in the lung was verified by in vivo optical imaging $24 \mathrm{~h}$ after their instillation. Following in vivo optical imaging mice were sacrificed and prepared for phase contrast CT scanning in the way described above. I was able to reproduce the results of the first study, showing that the quality of the obtained data sets allowed a quantification of structural alterations within the lung and in doing so the discrimination between asthmatics and controls. But moreover, we were able to identify clusters of injected macrophages due to their enhanced x-ray absorption by the phagocytized $\mathrm{BaSO}_{4}$ (Figure 9). In these phase contrast CT data sets we found a much higher amount of these cells in the asthmatic compared to the healthy lung tissue. In order to confirm these results NIRF microscopy and high resolution phase contrast x-ray microscopy was performed on lung sections from the same animal model. Both techniques also revealed the presence of the labeled cells, but did not demonstrate a strong difference in their total amount between asthmatic and healthy lung tissue. However, only in the asthmatic tissue clusters of cells including our instilled macrophages were detected. Hence single distributed labeled MH-S cells cannot be detected with our in-situ lung imaging setup these clusters most probably caused the observed higher concentration of Barium labeled cells in asthmatic lung tissue (Figure 9). 

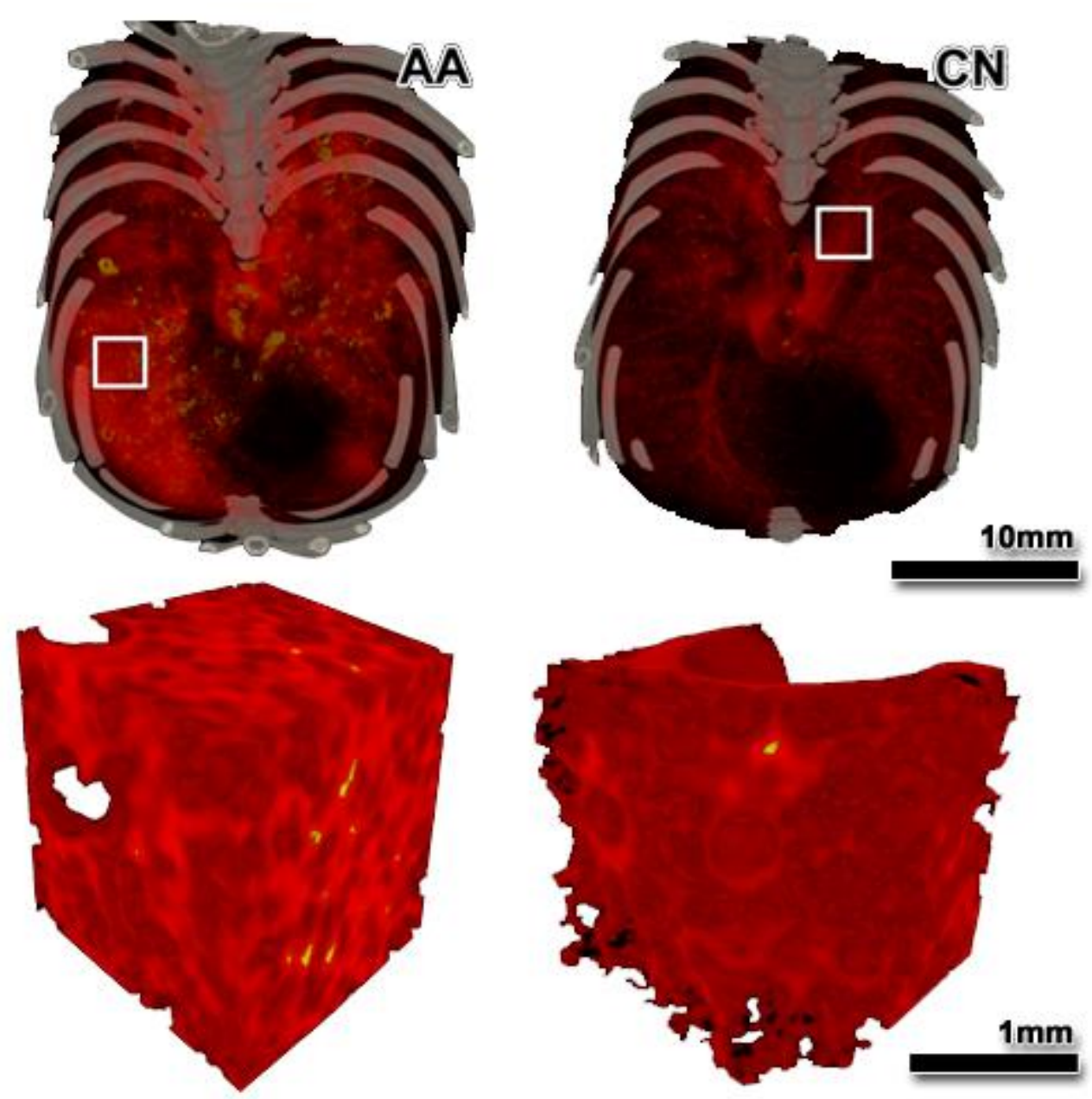

Figure 9: 3D localization of the barium sulfate labeled macrophages within the lung:

in Volume rendering representation of the phase contrast CT data sets of an asthmatic mouse (AA) and of a healthy control (CN), grey = bones red $=$ soft tissue, gold $=$ Barium. VOIs $\left(1 \times 1 \times 1 \mathrm{~mm}^{3}\right)$ are shown for the location indicated by the white rectangle.

\section{\{SOURCE: Dullin et al.[21] modified\}}

In summary, I demonstrated for the first time that functional CT imaging, exploiting labeled macrophages as specific carriers of a CT contrast agent, is feasible in in-situ lung imaging of asthmatic mice and healthy controls.

\section{5) PHASE CONTRAST ZOOM-TOMOGRAPHY REVEALS DETAILED LOCATION OF MACROPHAGES IN MOUSE LUNGS}

M. Krenkel, A. Markus, M. Bartels, C. Dullin, F. Alves, T. Salditt, "Phase contrast zoom-tomography reveals detailed location of macrophages in mouse lungs," Scientific reports 5, 2015

In order to study more precisely the fate of the instilled labeled MH-S cells, imaging with a higher spatial resolution was required. To this end, the study was repeated, but instead of our sample preparation scheme used before, the lungs were explanted after inflation with paraformaldehyde, embedded in agarose gel and cut into $500 \mu \mathrm{m}$ thick slices. These samples were then analyzed at the ID 22 beamline (ESRF, Grenoble France) by Martin Krenkel from the institute for x-ray physics of 
the University Göttingen headed by Prof. Tim Salditt. He used a setup for x-ray holotomography utilizing wave-guides developed within the group of Prof. Salditt. Phase retrieval was performed in the obtained data resulting in data sets with a voxel size of $430 \mathrm{~nm}$. At this subcellular spatial resolution single labeled macrophages could be easily identified within the local lung structure environment (Figure 10). Due to the very complex setup, the long acquisition time and the limited viability of ID 22 only a few samples taken from two controls, two asthmatic mice and one mouse without injected macrophages (serving as negative control) were analyzed. The high resolution also limits the available field of view so that only manually chosen regions of the samples were scanned at this level of detail. However, the remarkable feature of this approach is that the magnification can be varied, thus allowing to zoom into certain sites of interest. The generated data sets showed no obvious differences in the amount of labeled macrophages or in their localization between the different groups.

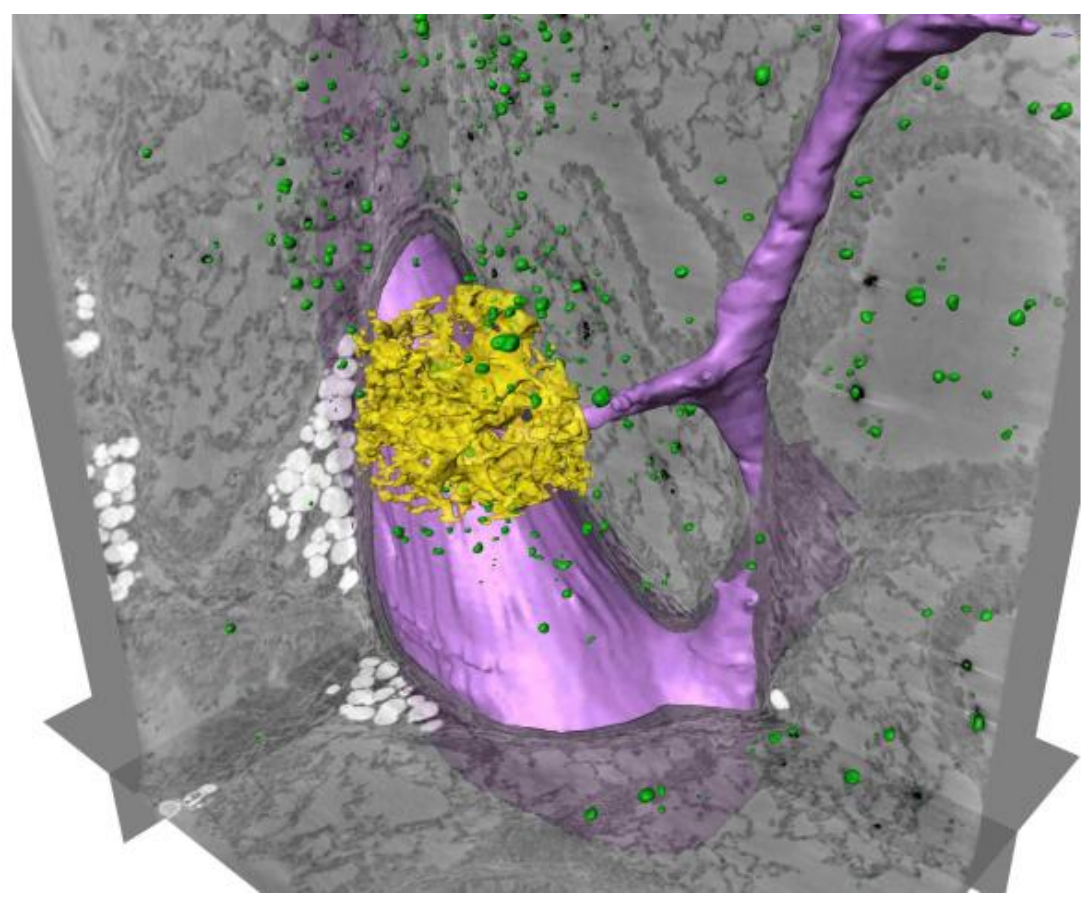

Figure 10: 3D localization of the labeled macrophages in a thick lung section scanned by holotomography: three orthogonal oriented slices are shown together with automatically labeled Barium clusters (green) and alveolar walls in a small ROI (yellow). A part of a blood vessel has been marked semi automatically (purple).

\{SOURCE: Krenkel M, et al. [24], modified\}

\section{B. LUNG FUNCTION MEASUREMENT}

All the methods demonstrated in this thesis so far measures secondary effects in asthma. Up to now, it is not known to which extent these hallmarks are related to the clinically most interesting parameter which is the "lung function". In the clinic, the direct assessment of lung function is the gold standard for the characterization of asthmatic patients. In preclinical research lung function assessment is usually done by plethysmography, a technique that measures the pressure differences caused by breathing. In most cases, plethysmography goes along with the immobilization or 
intubation of the mouse. Plethysmography can also be performed on freely breathing mice, but the reliability of this approach is heavily discussed [16]. As an alternative, a method analyzing video streams of mice placed in a transparent box was introduced by Bates et al. [29]. Although this approach claims to allow the measurement of lung function in free moving mice, the described box in which the mouse is kept during the acquisition is so small that the mouse is still very restrained, thus leading to potential higher stress levels of the animal. As an increased stress level, especially in asthma, will influence the results, my aim was to develop an alternative approach to assess lung function.

\section{1) QUANTIFICATION OF LUNG FUNCTION IN PRECLINICAL ASTHMA MOUSE MODELS BY PLANAR CINEMATIC X-RAY IMAGING}

In humans, "air-trapping", meaning the inability to expel the inhaled air from certain areas of the lung, is used to identify the presence of asthma by CT. The trapped air lowers the average x-ray attenuation and as a result these regions appear darker than normal ventilated areas on CT scans in expiration state. In order to reliably measure such an effect in mice, CT with a sufficient spatial resolution is required. As previously discussed, such a resolution in the range of microns would be accompanied with an increased x-ray dose that prohibits an in vivo application. In addition, inhaling and expelling of air cannot be controlled in mice. In order to reconstruct the lung either in inspiration or expiration state a gated imaging approach is necessary. For this purpose retrospective gating is usually used in in vivo small animal CT mainly [30], where instead of one set of projection images several rotations are acquired to retrospectively generate one data set for the same state of the chest. The advantage of this approach is that any CT device that is capable of acquiring more than one rotation can be used. However, performing several rotations increases the overall scanning time and applies several times the dose of one "classical" CT scan to the studied object/subject. Therefore, there was the strong need to acquire a better method to address and quantify an impairment of lung function in mice.

This inspired me to develop a method to measure lung function based on planar cinematic x-ray imaging. With planar chest radiography the lung can be easily visualized, an effect which has been used for medical diagnosis of lung disease for decades. By utilizing the low dose in vivo microCT "QuantumFX" (Perkin Elmer), x-ray movies with 1024 frames at a frame rate of $30 \mathrm{~Hz}$ can be recorded. Applied to the chest region of a mouse this means that $34 \mathrm{~s}$ of the breathing cycles can be imaged. Since the changes in the air content due to breathing cause significant modulations in the $x$ ray attenuation over the lung area, the function generated by measuring the average $x$-ray attenuation of the lung (XAF) will contain information about alteration of the lung function. As demonstrated for three mice in Figure 11, the XAF shows distinct breathing events for a healthy mouse (blue), an asthmatic mouse (red) and a dexamethasone (DEX) treated asthmatic mouse (green). Whereas the blue and green curves look very similar, the red curve is characterized by a higher baseline, as well as by much smaller and asymmetric peaks. Within this thesis I developed a method to quantify these breathing events by fitting a second order polynomial function in the peaks of the XAF. For each mouse and each breathing event the parameters of this polynomial function $\left(\mathrm{XAF}^{\prime}(\mathrm{t})=\mathbf{Q} \mathbf{F}^{*} \mathrm{t}^{\wedge} 2+\mathrm{k}^{*} \mathrm{t}^{\wedge} 1+\mathbf{C}\right)$ and additionally the width of the breathing event (L), the area under the curve (AuC) as well as a symmetry index (AnIso) are calculated and averaged per mouse. 
a)

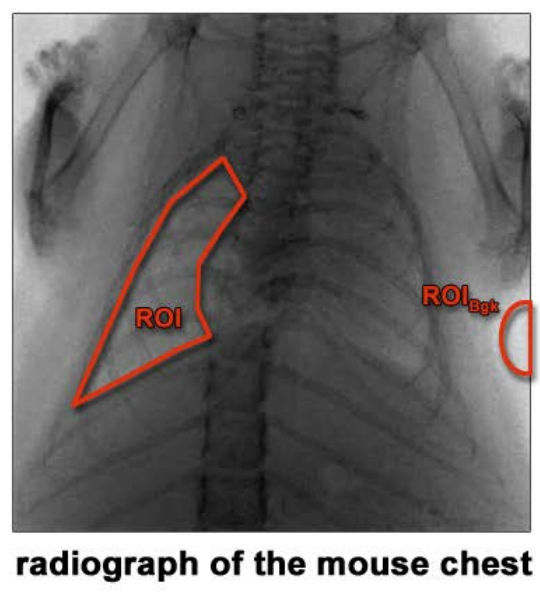

\section{b)}

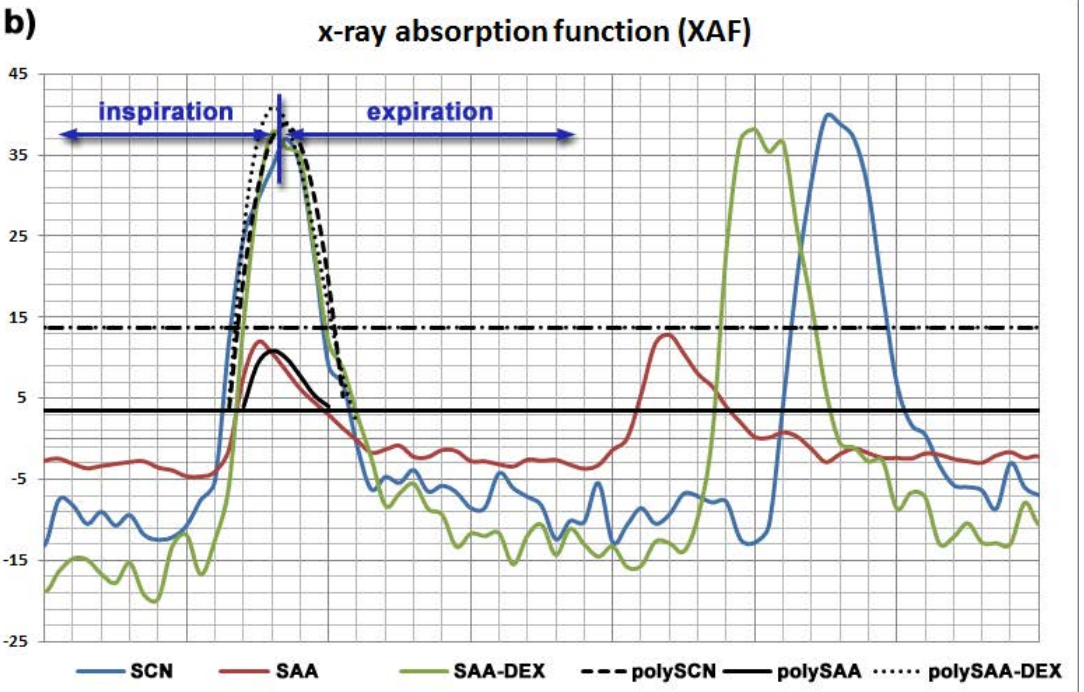

Figure 11 Principle of the in-vivo lung function measurement approach based on cinematic planar $x$-ray imaging:

a) shows the imaged chest region of the mouse. The changes in the x-ray absorption function (XAF) were measured as average grey values over the right side of the lung (ROI) and normalized by the background region ROIBgk. b) shows exemplified the XAFs of a healthy control mouse (SCN, blue), an asthmatic mouse (SAA, red) and a dexamethasone treated mouse (SAA-DEX, green). Clearly a dramatically reduced area under the curve can be seen for SAA in comparison to both the healthy and the treated mouse, pointing to a reduced volume of transported air in the asthmatic mouse. In addition the XAF of the asthmatic mouse shows a slower decay, reflecting a reduced elasticity of the lung tissue and therefore a reduced speed of expelling the inhaled air. For quantification level functions were calculated automatically (horizontal lines). Between two intersection points of the level functions with the XAF a polynomial function of the order 2 was fitted into the XAF (dashed, dotted and solid interpolation function).

In order to establish this procedure, in a first step the chest regions of isoflurane-anesthetized and therefore non-moving mice were imaged. However, combined with a motion tracking algorithm, the method has even the potential of analyzing free moving mice as well.

In order to verify the robustness of this novel technique, it was applied on healthy BALB/c mice of both genders and of different age. In order to access age related effects the mice were divided into three groups: a) younger than 7 weeks, b) between 7 and 11 weeks of age and c) older than 11 weeks. A reduction of $\mathbf{Q F}$ (the factor of the quadratic term of the fitting polynomial function) and an increase of $\mathbf{L}$ (the average width of the breathing events) with increasing age of the mice were found. However, I could not identify significant gender related variations besides a slightly increased $\mathbf{L}$ has been found in male mice. This latter effect is most likely related to the fact that male mice are larger than females of the same age. This indicates that only age and gender matched mice should be used. In need to compare results from studies performed with mice of different age, only the relative difference between the parameters for asthmatic and control mice should be used.

In order to demonstrate that this method can be used with a low x-ray dose and is therefore suitable for in vivo studies, a set of mice was measured with varying $\mathrm{x}$-ray doses. In this experiment a relationship between the applied dose and the calculated parameters was not found, thus indicating that even with in a very low dose setting of about $6.5 \mathrm{mGy}$ per acquisition $(90 \mathrm{kV}$ and $40 \mu \mathrm{A})$ the parameters of the XAF can still be evaluated. For comparison, the dose usually used to perform a CT scan at the same device (QuantumFX, Perkin Elmer, USA) that covers roughly half of the mouse is 
about 40 mGy (which is still very low compared to other systems on the market). Since similar CT scans have been performed by us once per week in longitudinal tumor therapy studies in mice and have never caused any measurable side effects, it can be assumed that the lung function measurement technique which uses about $16 \%$ of this dose can be applied unreserved in in vivo longitudinal studies.

I could demonstrate that the introduced lung function parameters $(\mathbf{Q F}, \mathbf{C}$ and $\mathbf{L})$ can be used to discriminate between asthmatic and healthy mice. Moreover, I applied this method in mice of two asthma mouse models of different severity, DEX treated mice and healthy controls. For verification, the lungs were also analyzed by phase contrast CT and histology. Mice of all groups including the treated mice demonstrated remarkable differences in the analyzed parameter $(\mathbf{Q F}, \mathbf{C}$ and $\mathbf{L}$, Figure 12).
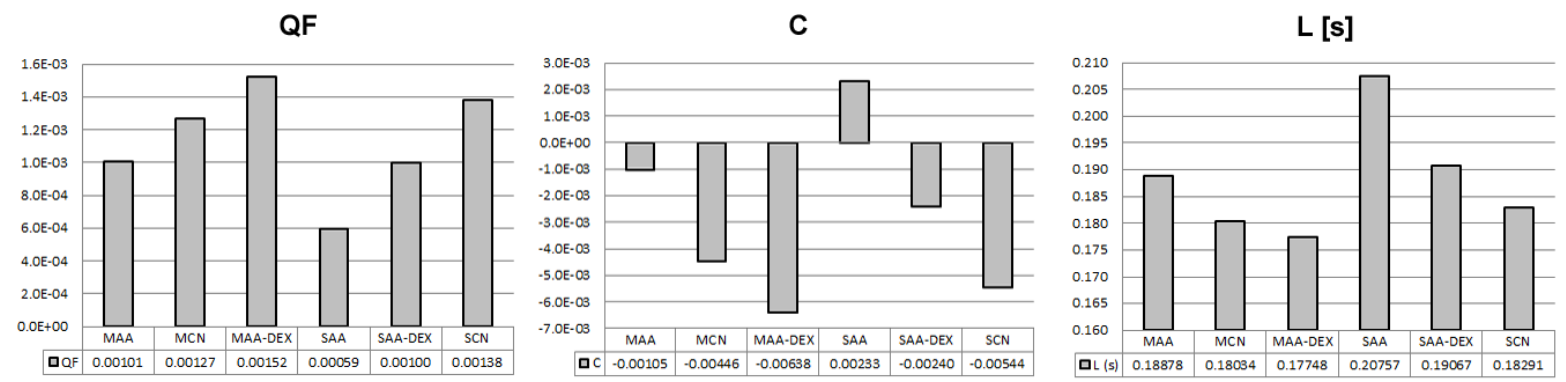

Figure 12 the obtained function parameters QF, C and $\mathrm{L}$ for the two asthma models, controls and DEX treated mice:

A reduced QF was found in asthmatic mice (MAA and SAA), where SAA shows a stronger reduction than the mild model MAA. Treated mice (MAA-DEX and SAA-DEX) showed a larger QF which in case of MAA-DEX exceeds the controls MCN (left). The same characteristic differences can been found in $\mathrm{C}$ and $\mathrm{L}$ (which represents the length of a breathing event). Asthmatic mice, especially from the severe model SAA, showed a prolonged breathing event as observed in human asthma patients as well. Also for this parameter the effect of the DEX treatment can be seen, as a shortening of the breathing event time towards the one of healthy animals (SAA-DEX) or, in case of MAADEX, exceeding the controls.

In the presence of asthma a reduction of $\mathbf{Q F}$, an increase of $\mathbf{C}$ and a prolongation of $\mathbf{L}$ was observed. Since $\mathbf{Q F}$ is the parameter responsible for the steepness of the polynomial function, a reduction means a smaller interval of x-ray attenuation changes within the lung, thus pointing to an impaired exchange of air. $\mathbf{C}$ basically measures the baseline of the polynomial function and therefore its increase demonstrates the air trapping effect in asthma. $\mathbf{L}$ is the length of a breathing event showing the expected effect of extended expiration times in asthmatic mice due to the reduced elasticity of the lung tissue. It can be seen that these parameters, in accordance with the design of the experiment, showed a reduced lung function in the asthmatic animals (stronger in the once from the severe model) compared to the healthy controls and intermediate values for the treated animals. In the case of the mild asthma model the treated mice even expressed a "better" lung function than the healthy controls, suggesting that the applied DEX dosage (causing bronchodilation) may overcompensate the negative effects of asthma. Overall, the method proved to be a reliable measure of lung function able to discriminate between asthmatic mice of different severity as well as, treated and healthy mice. In order to verify and correlate the results phase contrast CT and Histology (H\&E and PAS staining) has been performed in the same animals ex vivo. A detailed discussion of the correlation between the parameters of the different imaging techniques is presented in chapter $C$. 
Furthermore using this lung function measurement technique, I demonstrate that the natural recovery from the disease in mice from the mild asthma model can be analyzed. For this, asthmatic and healthy control mice were measured 2 days and 16 days after the asthma attack (Figure 13). Interestingly, in the recovered asthmatic mice the length of the breathing events $\mathbf{L}$ as well as the height of the baseline $\mathbf{C}$ showed no difference to the controls anymore, whereas $\mathbf{Q F}$ and AuC remained reduced and AnIso increased. This may indicate that there is no longer any acute air trapping in the mice that recovered from asthma. However, the lung function seems still to be impaired due to persistent changes in the lung tissue elasticity, which is also known for asthmatic patients [31](shown by the AnIso parameter). Since phase contrast CT analysis on recovered mice did not reveal any differences to healthy mice, the lung function measurement method proposed in this thesis provides even additional information and proved to be more sensitive than CT.

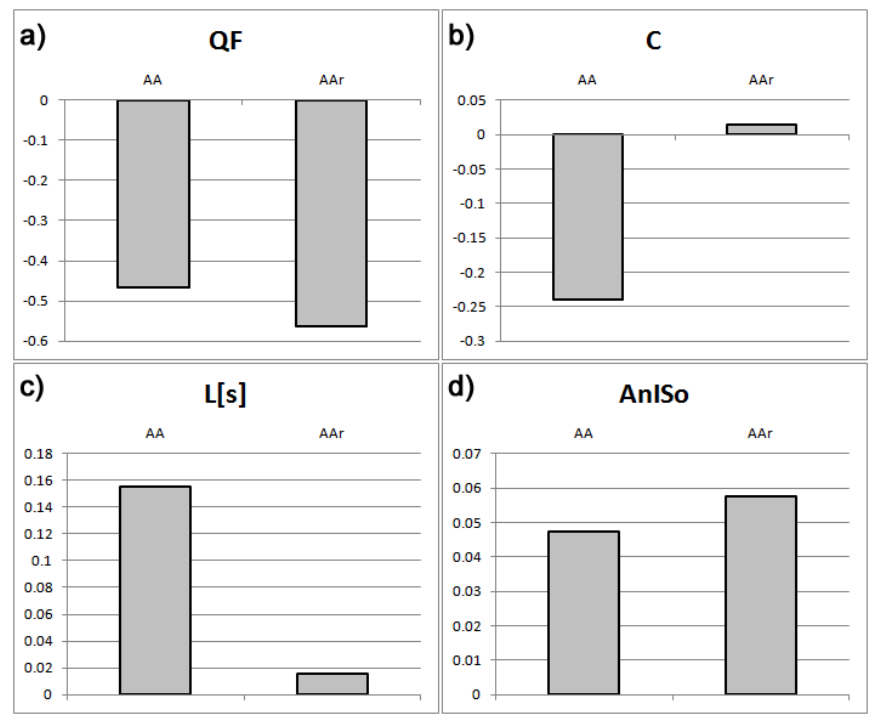

FIGURE 13 Recovery of asthmatic mice assessed by lung function measurements:

The relative difference of the lung function parameter in between asthmatic mice 2 days (AA) and 16 days (AAr) after the last OVA challenge compared with healthy controls. Therefore 0 equals healthy conditions. a) shows that still in AAr the parameter QF (steepness of the breathing peak) is strongly reduced, b) instead indicates that the baseline parameter $\mathrm{C}$ has basically reached normal condition also c) the length of the breathing peak equals healthy control mice. However d) demonstrate that anisotropy index AnIso remains different to the controls.

It has to be noted that the analysis is influenced by the breathing frequency and therefore it was adjusted to about one breathing event in $1.4 \mathrm{~s}$ by modifying the isoflurane concentration. The used anesthesia device is not optimized for such an approach and therefore the obtained synchronization of the breathing frequencies was not optimal. This may account for the fact that the lung function parameters showed stronger variations within each group than the results obtained for instance by phase contrast CT and histology. If in future an electronic controlled anesthesia device will be used this problem will be circumvented.

In summary, I demonstrated that the measurement of lung function based on low-dose planar cinematic x-ray imaging is feasible and sensitive enough to discriminate between healthy mice, mice from different asthma models and treated mice. The method can be used in in vivo longitudinal studies due to the low x-ray dose. Therefore, this technique provides a functional readout allowing the quantification of this important clinical symptom - the impairment of lung function - and its 
correlation to alterations on the anatomical and molecular level within the mouse lung. Since the lung function is also influenced by changes in the lung tissue elasticity, this approach provides a direct measure of this effect which is missing in the CT based analysis. While only demonstrated on anesthetized mice, the application of our method on free moving non-anesthetized mice seems to be feasible and will be topic for further developments to improve this approach.

\section{CORRELATION BETWEEN THE ANATOMICAL AND FUNCTIONAL MEASURES}

So far, the two routes - anatomical and functional imaging were discussed separately. For a better understanding of asthma, the course of the disease or the treatment response, the relationship between the different parameters is of major interest. For this purpose, we conducted a study in which asthmatic mice (MAA and SAA), DEX treated mice as well as healthy controls were analyzed in vivo by $\mathrm{X}$-ray based lung function measurement and ex vivo by phase contrast CT. Furthermore, histology was performed to validate the obtained results. Please note, that a control group for each asthma model (MCN and SCN) was used. These groups received PBS instead of OVA at the same time points as the respective asthma mice. Moreover, the preparation of the lungs for histology was done after the phase contrast CT scans. In this case the lungs were kept in-situ without fixatives for up to 5 days, which might explain the reduced quality of the obtained histological sections. Therefore, direct quantification of hallmarks of asthma such as airway wall thickening was not able to perform.

For histology, haematoxylin eosin (H\&E) and periodic acid-schiff (PAS) staining were performed to assess hallmarks of asthma by identifying the grade of immune cell infiltration and the amount of mucus production. Histological sections of all mouse lungs were scored by four independent researchers using a scale from 0 (no visible immune cell infiltration or no mucus) to 3 (large amount of cell infiltration or strong mucus production). Since the H\&E score represents the increase of immune cells in the lungs, it is not surprising that even airway wall thickening is not addressed, due to the limited quality of the histological sections, the results correlate with an increase in the soft tissue vol. ratio obtained by phase contrast CT (Figure 14). When comparing the soft tissue vol. ratio with the corresponding normalized $\delta$-value (the real part of the refractive index responsible for the phase shift) (Figure 14a), in the presence of asthma both, the soft tissue ratio and the $\delta$-value were found to be increased. The first parameter quantifies airway wall thickening and immune cell infiltration the latter indicates a modification of the cell-to-water ratio within the lung tissue, which both are well known effects of human asthma. Also the response to a DEX treatment was quantified, which significantly reduces the soft tissue vol. ratio by bronchodilatation, but does not change the composition of the lung tissue ( $\delta$-value remains high). Interestingly, if the DEX treated mice were excluded, Figure 14b) shows that the H\&E score correlates with the normalized $\delta$-value and reflects the severity of asthma. In the treated mice MAA-DEX and SAA-DEX the H\&E score is reduced but the $\delta$-value remains high. This might indicate that the amount of infiltrating cells can be reduced by DEX but there must be other cells or enlarged cells involved that keep the $\delta$-value up. Figure 14c) shows a relationship of the soft tissue vol. ratio to the amount of infiltrating cells stated by the H\&E score. It should be noted that the scores are not linear and therefore a linear correlation cannot be expected. Figure 14d) proves that both the H\&E and PAS score are reliable measurements to assess the severity of asthma. From this figure we can also conclude that in SAA the applied treatment was not sufficient to completely undo the alterations caused by asthma, whereas in MAA-DEX no significant difference to the healthy controls can be observed. Since PAS and H\&E scores are highly 
correlated, Figure 14e) and f) demonstrate the same trends as in Figure 14b) and c). Based on visual comparison the different groups seem to be a bit more separated in Figure 14e) and f) than in b) and c) suggesting that the PAS score is slightly more sensitive to discriminate between mice of different groups.
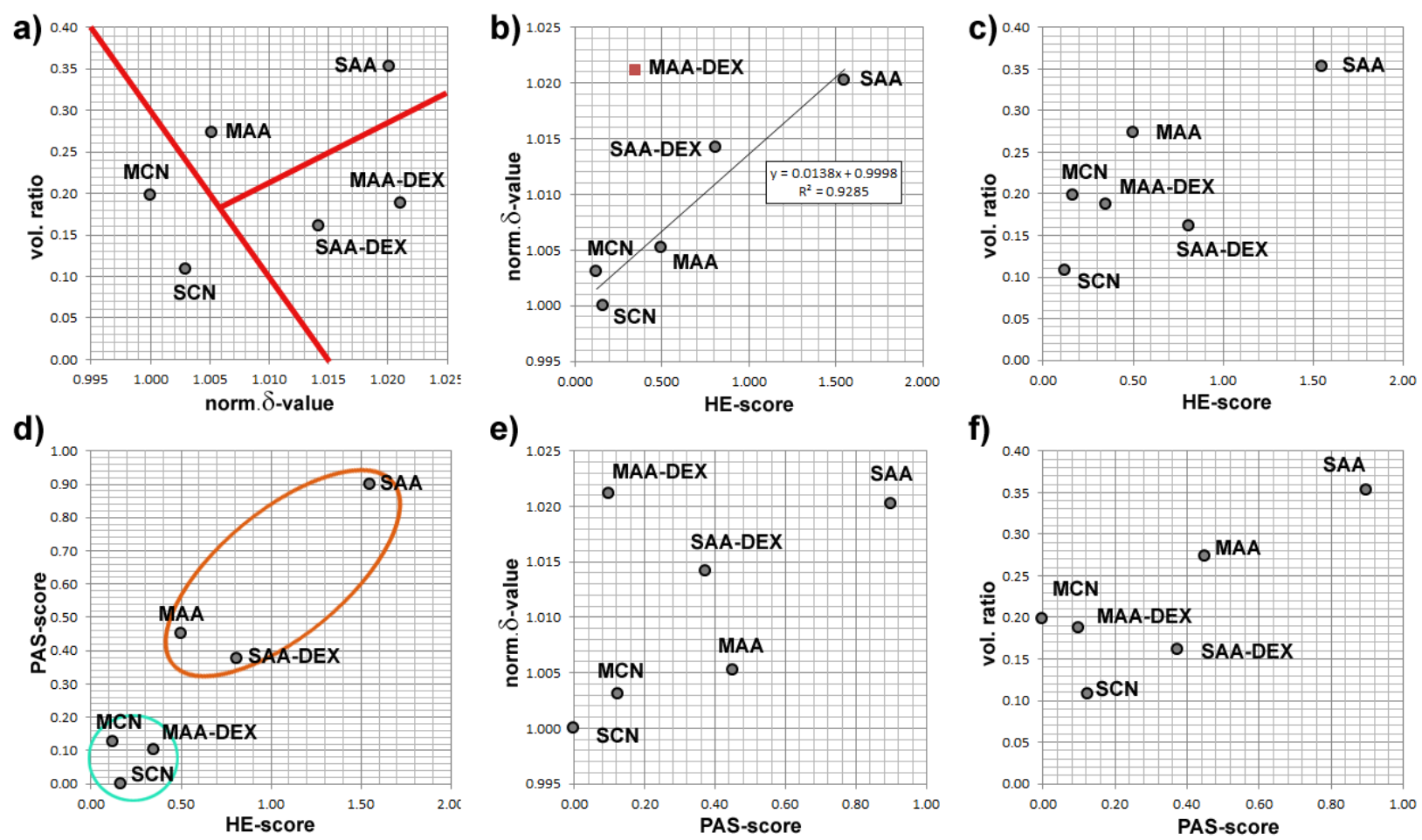

Figure 14 Correlation between phase contrast CT and histology:

The various plots a)-f) display the correlation between two individual parameter. a) indicates that asthma is characterized by both a higher vol. ratio and an increased normalized $\delta$-value of the lung tissue. Interestingly the treatment seems to reduce the vol. ratio (so it treats the swelling of the tissue) but does not chance the altered chemical composition of the lung tissue (the normalized $\delta$-value is not reduced). It can be also seen (indicated by the red lines) that the combination of vol. ratio and d-value allows a clear separation into asthmatic, treated and healthy mice b) shows that there is a high degree of correlation between cell infiltration (H\&E) and normalized $\delta$ value, thus meaning that more cells within the lung tissue increase the obtained d-value. This relationship is disturbed in the presence of treatment. c) shows that the bronchodilation caused by the DEX treatment yields to a lower vol. ratio but does not change the amount of infiltrating cells that much. d) indicates that both PAS and H\&E scores are reliable measures of asthma and therefore show a high degree of correlation. It can be also seen that in case of the severe model the DEX treatment was not completely successful and therefore SAADEX appears grouped together with SAA and MAA. Since H\&E and PAS score are highly correlated e) and f) show the same trends like b) and c).

Another important issue is the relationship between anatomical information and lung function, as this would demonstrate how predictive a morphological analysis in asthma mouse models is. These results are demonstrated in Figure 15, showing that asthmatic mice can be easily discriminated from healthy and treated mice by combining the soft tissue vol. ratio obtained by phase contrast CT with the measured lung function parameter $\mathbf{Q F}, \mathbf{L}$ and $\mathbf{C}$. As the two asthma models were setup in parallel using age and gender matched mice, the same results were expected for the two control groups MCN and SCN. The only difference in the setup of the control mice was the schedule in which they received PBS. Since MCN and SCN can be clearly separated, I believe that injection of even PBS alone, can result in an alteration of the lung tissue, thereby suggesting that great care needs to be 
taken when comparing results obtained in different asthma models. This finding needs further evaluation.
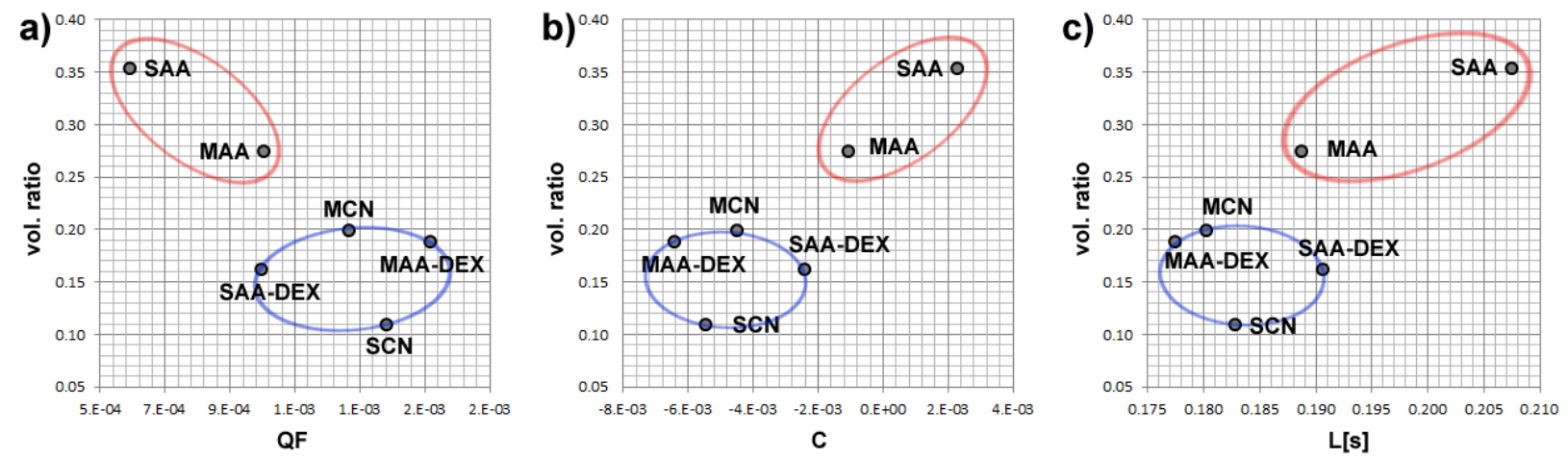

Figure 15 relationship between phase contrast CT (vol. ratio) and the lung function parameter (QF, C and L[s]): Since the lung function parameters $\mathrm{QF}, \mathrm{C}$ and $\mathrm{L}[\mathrm{s}]$ are all changed in asthma and show a high degree of correlation, very similar trends can be seen in a), b) and c). Whereas, based on solely the lung function parameter for instance MAA cannot easily separated from SAA-DEX, the combination of phase contrast CT and lung function measurement would allow a clear discrimination as indicated by the marked regions.

In summary, I could show that the established histological analysis of lung tissue from asthmatic mice, although limited to 2D, shows the same trends like the lung tissue vol. ratio parameter obtained by phase contrast CT. Additionally, phase contrast CT correlates well with the lung function measures, which indicates a strong relationship between structural and functional alterations in asthma. The observed correlations may also account for the trustworthiness of the two novel approaches for the analysis of asthma mouse models: phase contrast CT and x-ray based lung function measurement. Interestingly, the differences that we found in the two asthma models suggest, due to the different induction schemes, additional effects rather than only increasing magnitude of the hallmarks of asthma. Moreover, the DEX treated mice displayed different features than the controls, which may indicate that the treatment only suppresses some symptoms but does not cure the asthmatic mice.

\section{MOLECULAR IMAGING}

In order not to limit the analysis of asthma in preclinical mouse models to the quantification and classification of symptoms, but also to study the underlying molecular processes such as cell-cell interaction and cell migration, molecular imaging by means of in vivo near infrared fluorescence (NIRF) imaging was performed. Particularly in rather dynamic diseases such as asthma, which demonstrate different phases of an immune response, a method with a fast readout such as NIRF imaging would be very useful to monitor the course of the disease as well as to assess treatment response. However, structural changes as well as changes in the lung function in asthmatic mice cannot be measured due to the limited spatial and temporal resolution of such systems, especially of systems using a raster acquisition scheme. Thus, NIRF optical imaging is either focused on the inflammatory progression of asthma or on the detection of differences in population or recruitment of specific cell types to the lung. 


\section{1) DENDRITIC POLYGLYCEROLSULFATE NEAR INFRARED FLUORESCENT (NIRF) DYE CONJUGATE FOR NON-INVASIVELY MONITORING OF INFLAMMATION IN AN ALLERGIC ASTHMA MOUSE MODEL}

S. Biffit, S. Dal Monego†, C. Dullin, C. Garrovo, B. Bosnjak, K. Licha, P. Welker, M. M. Epstein, and F. Alves, "Dendritic polyglycerolsulfate near infrared fluorescent (NIRF) dye conjugate for non-invasively monitoring of inflammation in an allergic asthma mouse model," PloS One, vol. 8, no. 2, p. e57150, 2013.

$\dagger$ equal contribution

Asthma causes a local inflammation within the lung which can be exploited for the detection of the disease by using optical imaging in combination with smart fluorescence probes as demonstrated by Cortez-Retamonzo et al. [19]. However, these smart probes (ProSense and MMPSense) which are activated predominately by either Cathepsin-B or MMP-9 and MMP-13, share the same disadvantages: a rather slow kinetic and the lack of attachment to the site of activation, creating a substantial background signal. Both effects hamper the applicability of this kind of probes in asthma mouse models. Therefore, we applied a novel dendritic polyglycerolsulfate NIRF dye conjugate (MN2012) [32]. This dye is known to be taken up by macrophages and to bind P- and L-selectin. Following intravenous injection of MN2012 the average fluorescence intensity was measured over the chest area. We demonstrated that MN2012 accumulates in the lung and showed the highest difference between asthmatic and control mice $4 \mathrm{~h}$ after the administration of the dye, in comparison to $24 \mathrm{~h}$ with above mentioned commercial smart probes which peak later due to their activation process. In summary, in this study we concluded, that the use of MN2012 is beneficial especially if a faster readout of changes in the local inflammation is needed, for instance to monitor a given treatment. However, we also found that the commercial dyes, despite their slow kinetic, expressed a much stronger signal. Since MN2012 has also proven to act anti-inflammatory at higher doses [33], increasing the dose to match the signal intensity of the commercial dyes may affect the asthma model and is therefore contraindicated. Thus, in applications in which only weak signals closer to the detection limit of the optical imaging system are expected, the use of commercial dyes instead of MN2012 seems preferable.

\section{2) NON-INVASIVE OPTICAL IMAGING OF EOSINOPHILIA DURING THE COURSE OF AN EXPERIMENTAL ALLERGIC AIRWAYS DISEASE MODEL AND IN RESPONSE TO THERAPY}

M. A. Markus, C. Dullin, M. Mitkovski, E. Prieschl-Grassauer, M. M. Epstein, and F. Alves, “Non-Invasive Optical Imaging of Eosinophilia during the Course of an Experimental Allergic Airways Disease Model and in Response to Therapy," PloS One, vol. 9, no. 2, p. e90017, 2014.

The imaging strategy discussed above, using Cathepsin-B, MMP-9 and MMP-13 sensitive probes or MN2012 presents a readout for inflammation independent of the underlying disease and is therefore not specific for asthma. To this end, we focused on another hallmark of asthma: the increase of the total amount of eosinophils within the lung during an asthmatic response (eosinophilia). Eosinophilia is reduced by most of the applied therapeutic concepts such as DEX based therapies. Therefore, imaging eosinophils within the lung would allow to identify the 
presence of asthma as well as to monitor treatment efficacy. Here, we used a NIRF-labeled antiSiglec-F antibody. Siglec-F is expressed on the membrane of eosinophils and to a much lower extent on macrophages. In this study a significant difference in the amount of eosinophils between asthmatic and control mice was found in the lung region at $48 \mathrm{~h}$ and $72 \mathrm{~h}$ after administration of the NIRF-labeled anti-Siglec-F antibody. Moreover, asthmatic mice treated either with DEX or betaescrin, a new anti-inflammatory drug derived from Chinese horse chestnut seeds, expressed an about 2-fold decreased fluorescence intensity, indicating that this approach can be used to detect treatment response. Additionally, the results were validated by utilizing ex vivo NIRF microscopy, bronchoalveolar lavage (BAL) and classical histology. In summary, we demonstrated for the first time that eosinophilia can be monitored in vivo and non-invasively in asthma mouse models and that this NIRF imaging approach can be used to assess preclinically treatment response over time.

3) TRACKING OF INHALED NEAR-INFRARED NANOPARTICLES IN LUNGS OF SKH-1 MICE WITH ALLERGIC AIRWAY INFLAMMATION

A. Markus, J. Napp, T. Behnke, M. Mitkovski, C. Dullin, S. Kilfeather, U. Resch-Genger, and F. Alves, "Tracking of inhaled near-infrared nanoparticles in lungs of SKH-1 mice with allergic airway inflammation" ACS Nano, in revision

Cell migration, especially of macrophages, is of great interest as it has been shown by Mizue and Chen [28], [34] that inhibition of the macrophage migration inhibition factor (MIF) prevents airway remodeling and therefore the development of asthmatic symptoms. In order to study the migration behavior of endogenous macrophages, we used NIRF nanoparticles which we intranasally instilled into mice. In this paper we show that inhaled NIRF (Itrybe) nanoparticles are predominately taken up by alveolar M2 macrophages (AMs) in the peribronchial and alveolar space, allowing for in vivo detection of these cells by means of NIRF imaging. We found higher fluorescence intensity in the lung area of mice from an OVA induced allergic airway disease model in comparison with healthy controls. These findings were validated by confocal microscopy of lung tissue sections co-stained with a fluorescent labeled anti-CD68 antibody. In summary, we demonstrated that in vivo NIRF imaging can be used to investigate the fate of inhaled NIRF nanoparticles within the lungs of asthma mice which may also aid the analysis of the biodistribution of inhaled therapeutics in asthma. Since the Itrybe particles were predominately found in M2 macrophages, the approach can be used in other disease models to perform tracking of endogenous M2 macrophages.

\section{SUMMARY OF THE MOLECULAR IMAGING PART}

In the here presented studies we showed that NIRF imaging, despite its poor spatial resolution, is a valuable tool to detect asthma in mouse models, to monitor certain aspects like the degree of inflammation or the recruitment of specific cell into the lung and more importantly to address treatment response in a living mouse over time. It has been shown that the alternative dye MN2012 can be used to detect inflammation during asthma and expresses a faster kinetic than the so far used commercial smart probes. We also demonstrated that specific cells can be labeled either by antibody-dye conjugates or by uptake of NIRF nanoparticles and, even though single cell tracking is not feasible, the differences in their total amount can be monitored over time within the lung by in 
vivo NIRF imaging. Moreover, we showed that the population of specific endogenous cells such as eosinophils and M2 macrophages can be imaged, which will not only help to understand cell migration and cell-cell interactions in asthma, but could also aid to the development of more localized therapeutic approaches in asthma which may generate less side effects than systemic drug therapy.

\section{PROJECT SUMMARY \& FUTURE PLANS}

Within this thesis I demonstrated that despite the limiting small size of mouse lungs, cutting edge imaging techniques can provide detailed information regarding anatomical, functional and molecular alterations in lungs of mouse asthma models, thereby providing the necessary sensitivity to discriminate between mice with asthma of different severity as well as between DEX treated, disease recovered and healthy mice.

I established and validated a novel approach for anatomical imaging of in-situ mouse lungs based on a combination of Synchrotron inline free propagation phase contrast CT and single distance phase retrieval that allowed a reliable quantification of subtle morphologic alterations within the lung tissue of asthmatic mice such as increased soft tissue vol. ratio. This approach has been proven to be sufficient to discriminate between mice with asthma of different severity, DEX treated and healthy mice. Moreover, this technique was sensitive enough to visualize barium sulfate labeled macrophages that were intra tracheal instilled into the lungs and thereby depict in-situ differences in the bio distribution of macrophages in lungs of asthmatic and healthy mice - the first approach of functionalized contrast in combination with CT lung imaging.

Furthermore, I invented a novel imaging approach to assess the impairment of lung function in asthmatic mice in vivo over time by low dose planar cinematic radiography. Utilizing this technique I was able to discriminate alterations of the lung functions in between mice from two asthmatic models, DEX treated mice, mice that had recovered from asthma and healthy controls. I demonstrated that this approach correlates well with different aspects of morphological alterations obtained by phase contrast CT and by histology. This new technique has been proven to be more sensitive than phase contrast CT when assessing treatment response. Moreover, I could show that with this method lung function can be measured without the need of intubating the mouse which may reduce its stress level.

In order to address molecular event within asthmatic mice, we successfully established three novel in vivo NIRF imaging strategies: first monitoring local inflammation within the lung with a new polyglycerol dye (MN2012) which has proven to be more specific discriminating asthmatic from healthy mice and to express a fast kinetic than the commercial smart probes, which will allow to follow the dynamic of an asthma attack more closely. Second, we demonstrated that an NIRF labeled anti-Siglec-F antibody can be used to visualize eosinophilia in vivo in asthma mouse models and will therefore provide an readout for treatment response on eosinophils. Third, we showed that we can image the fate of inhaled NIRF nanoparticles in vivo, which have been found to be predominately taken up by M2 macrophages. Therefore macrophages might be an interesting target for a nanoparticle based therapy. 
The combination of these novel imaging methods developed within this thesis creates an imaging platform that can be used to evaluate the response of novel asthma therapies based on their interactions with eosinophils, lung tissue and lung function in general. I demonstrated that these different techniques not only provide measures of established parameters such as airway obstruction and airway wall thickening, but also allow investigation of other features such as lung tissue elasticity measureable by direct evaluation of the lung function. By application of this imaging platform to assess the efficacy of a DEX therapy in an asthma mouse model, we found that eosinophilia, bronchial wall thickening as well as air-trapping could successfully be diminished by DEX, but alterations of the lung function were still present. Due to this and the fact that corticosteroid treatments such as DEX are known to produce side effects there is still a strong need for alternative therapies targeting asthma. The here developed imaging platform may aid to evaluation and optimization of such novel therapeutic strategies.

Within the scope of anatomical imaging, it is planned to use the high sensitivity of the generated phase contrast CT technique in combination with our preparation scheme to study the entire lung structure in-situ in great detail at different air pressures. This will allow the calculation of the local lung tissue elasticity, a parameter known to be substantially lowered in asthma. Persistent changes in lung tissue as discovered in patients even years after the last asthma attack, have to our knowledge never been the focus of a therapy approach before, but if cured would remarkably improve the wellbeing of the patients. These changes in the lung tissue elasticity might most likely not only be limited to the airways but could also affect the lung blood vessels. Changes in the blood vessel density in the presence of asthma have already been reported in the literature [35]. Therefore, I acquired data sets of lung tissue embedded in paraffin and stained with a tungsten containing acid (PTA), allowing the 3D-depiction of the lung blood vessels 3D in great detail. The data is currently being processed and will add another possible readout to the imaging platform.

The approach to use labelled macrophages for functional CT imaging in preclinical research, which has opened up possibilities for a broad range of applications due to the fact that it can be easily adapted to other inflammatory diseases. In addition, this also has the potential to exploit macrophages as carriers of therapeutics while monitoring their distribution in parallel.

The here presented lung function measurement will be in the focus of future research. It is planned to apply this strategy on free moving mice. The mice will be scanned in a box large enough to cause less stress and these boxes will in addition be used as houses inside the cages, so that the mice can adapt to it. That will hopefully allow the establishment of less stressful lung function measurements, thereby delivering more reliable results in preclinical asthma research. Hence, it is planned to perform a study comparing the novel technique with the established plethysmography and, in case of the x-ray based approach, on anaesthetized and unrestrained mice. This will reveal the potential benefit of the here presented lung function measurement approach and may also allow to match the calculated parameter (QF, L, C etc.) with established results from measurements with plethysmography. In addition, it will reveal the impact of the stress level on the obtained results.

The use of imaging platform generated in this thesis has already revealed unknown effects and features in asthma such as the migration pattern of the injected macrophages, the differences in the composition of the lung tissue in different asthma models, the inability of the applied DEX therapy 
to revert certain aspects of the lung function to healthy condition to only name a few. This not only opens up a large field of future research but may aid to a better understanding of asthma and could help to identify alternative targets for novel therapies and will therefore hopefully be beneficial for asthma patients in the future. 


\section{OWN CONTRIBUTION}

Due to the fact that this was a highly interdisciplinary and collaborative research project I would like to state in detail my contribution to the several subprojects:

1. Anatomical imaging

a. Accurate and precise reconstruction of complex refractive index in near-field single-distance propagation-based phase-contrast tomography

Phantom design, data acquisition, contribution to the writing process

b. Quantitative evaluation of a single-distance phase-retrieval method applied on in-line phasecontrast images of a mouse lung

Experimental idea and design, data acquisition, contribution to sample preparation, data analysis, generation of a novel method to assess edge sharpness, supervision of author S. Mohammadi/E. Larsson

c. Phase contrast CT for quantification of structural changes in lungs of asthma mouse models of different severity

Experimental idea and design, data acquisition, data analysis, writing of the publication, contribution to sample preparation

d. Functionalized synchrotron in-line phase-contrast computed tomography: a novel approach for simultaneous quantification of structural alterations and localization of barium-labelled alveolar macrophages within mouse lung samples

Experimental idea and design, data acquisition, contribution to data analysis and sample preparation, supervision of E. Larsson in the 3D data analysis process

e. Phase contrast zoom-tomography reveals detailed location of macrophages in mouse lungs Involved in experimental design and sample preparation, contribution to writing process

2. Molecular imaging

a. Dendritic Polyglycerolsulfate Near Infrared Fluorescent (NIRF) Dye Conjugate for NonInvasively Monitoring of Inflammation in an Allergic Asthma Mouse Model

Experimental design, analysis strategy, contribution to data acquisition, involved in writing of the publication

b. Non-invasive optical imaging of eosinophilia during the course of an experimental allergic airways disease model and in response to therapy

Involved in data analysis and writing process

c. Tracking of inhaled near-infrared nanoparticles in lungs of SKH-1 mice with allergic airway inflammation

Involved in data analysis and writing process

3. Functional Imaging

a. Quantification of lung function in preclinical asthma mouse models by planar cinematic x-ray imaging

Experimental idea and design, data acquisition, analysis, writing of the publication

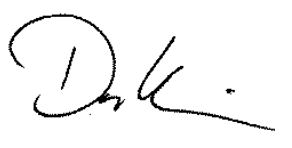

Christian Dullin

Göttingen, 20 October 2015 


\section{FUNDING}

This study was partly conducted within the European project 'Public Private Partnership for Asthma Imaging and Genomics' (P3AGI, www.p3agi.eu) funded by the European Commission through an FP7- IAPP Marie Curie Action (GA 230739) and was further funded by the 'Deutsche Forschungsgemeinschaft' (DFG, DU 1403/1-1) and the 'EXTREMA' COST action MP1207. The Synchrotron experiments were co-funded by the European program CALIPSO (Coordinated Access to Lightsources to Promote Standards and Optimization).

\section{ETHICS DECLARATION}

All presented imaging studies were performed in compliance with the guidelines of the European commission (86/609/EEC) the Italian (DL116/92) and the German ethical laws (33.9-42502-04$10 / 0134$ ) and were approved by either the Ministry of Health or by the animal ethics administration of Lower Saxony, Germany:

33.9-42502-04-10/0134 optical imaging with anti-Siglec-F antibody; phase contrast CT in mouse samples

33.12-42502-04-12/0834 tracking of inhaled near-infrared nanoparticles; holotomography

33.9-42502-04-15/1747 in-vivo lung function measurements in mice by planar cinematic xray imaging

\section{BIBLIOGRAPHY}

[1] S. S. Braman, "The global burden of asthma," Chest Journal, vol. 130, no. 1_suppl, p. 4S-12S, 2006.

[2] R. Beasley, "The Global Burden of Asthma Report, Global Initiative for Asthma (GINA)," 2004.

[3] H. Kelly, "Non-corticosteroid therapy for the long-term control of asthma.," Expert opinion on pharmacotherapy, vol. 8, no. 13, pp. 2077-2087, 2007.

[4] P. Bhavsar, M. Hew, N. Khorasani, A. Torrego, P. J. Barnes, I. Adcock, and K. F. Chung, "Relative corticosteroid insensitivity of alveolar macrophages in severe asthma compared with non-severe asthma," Thorax, vol. 63, no. 9, pp. 784-790, 2008.

[5] B. Fuchs and A. Braun, "Improved mouse models of allergy and allergic asthma-chances beyond ovalbumin," Current drug targets, vol. 9, no. 6, pp. 495-502, 2008.

[6] K. P. Leong and D. P. Huston, "Understanding the pathogenesis of allergic asthma using mouse models," Annals of Allergy, Asthma \& Immunology, vol. 87, no. 2, pp. 96-110, 2001.

[7] R. K. Kumar and P. S. Foster, "Are mouse models of asthma appropriate for investigating the pathogenesis of airway hyper-responsiveness?," Frontiers in physiology, vol. 3, 2012.

[8] M. M. Epstein, "Do mouse models of allergic asthma mimic clinical disease?," International archives of allergy and immunology, vol. 133, no. 1, pp. 84-100, 2004.

[9] N. L. Ford, M. M. Thornton, and D. W. Holdsworth, "Fundamental image quality limits for microcomputed tomography in small animals," Medical Physics, vol. 30, no. 11, p. 2869, 2003.

[10] D. M. Vasilescu, C. Klinge, L. Knudsen, L. Yin, G. Wang, E. R. Weibel, M. Ochs, and E. A. Hoffman, "Stereological assessment of mouse lung parenchyma via nondestructive, multiscale micro-CT 
imaging validated by light microscopic histology," J. Appl. Physiol., vol. 114, no. 6, pp. 716-724, Mar. 2013.

[11] A. Momose and J. Fukuda, "Phase-contrast radiographs of nonstained rat cerebellar specimen," Med Phys, vol. 22, no. 4, pp. 375-379, Apr. 1995.

[12] M. Kitchen, R. Lewis, N. Yagi, K. Uesugi, D. Paganin, S. Hooper, G. Adams, S. Jureczek, J. Singh, and C. Christensen, "Phase contrast $\mathrm{X}$-ray imaging of mice and rabbit lungs: a comparative study," The British journal of radiology, 2014.

[13] R. A. Lewis, N. Yagi, M. J. Kitchen, M. J. Morgan, D. Paganin, K. K. W. Siu, K. Pavlov, I. Williams, K. Uesugi, M. J. Wallace, C. J. Hall, J. Whitley, and S. B. Hooper, "Dynamic imaging of the lungs using $x-$ ray phase contrast," Physics in Medicine and Biology, vol. 50, no. 21, pp. 5031-5040, Nov. 2005.

[14] M. A. Beltran, D. M. Paganin, K. K. W. Siu, A. Fouras, S. B. Hooper, D. H. Reser, and M. J. Kitchen, "Interface-specific x-ray phase retrieval tomography of complex biological organs," Physics in Medicine and Biology, vol. 56, no. 23, pp. 7353-7369, Dec. 2011.

[15] A. V. Bronnikov, "Theory of quantitative phase-contrast computed tomography," p. 472, 2002.

[16] A. Adler, "Unrestrained plethysmography is an unreliable measure of airway responsiveness in BALB/c and C57BL/6 mice," Journal of Applied Physiology, vol. 97, no. 1, pp. 286-292, Mar. 2004.

[17] M. P. DeLorme and O. R. Moss, "Pulmonary function assessment by whole-body plethysmography in restrained versus unrestrained mice," Journal of pharmacological and toxicological methods, vol. 47, no. 1, pp. 1-10, 2002.

[18] J. Hickson, "In vivo optical imaging: preclinical applications and considerations," presented at the Urologic Oncology: Seminars and Original Investigations, 2009, vol. 27, pp. 295-297.

[19] V. Cortez-Retamozo, F. K. Swirski, P. Waterman, H. Yuan, J. L. Figueiredo, A. P. Newton, R. Upadhyay, C. Vinegoni, R. Kohler, and J. Blois, "Real-time assessment of inflammation and treatment response in a mouse model of allergic airway inflammation," The Journal of clinical investigation, vol. 118, no. 12, p. 4058, 2008.

[20] J. Haller, D. Hyde, N. Deliolanis, R. de Kleine, M. Niedre, and V. Ntziachristos, "Visualization of pulmonary inflammation using noninvasive fluorescence molecular imaging," Journal of Applied Physiology, vol. 104, no. 3, pp. 795-802, 2008.

[21] C. Dullin, S. dal Monego, E. Larsson, S. Mohammadi, M. Krenkel, C. Garrovo, S. Biffi, A. Lorenzon, A. Markus, and J. Napp, "Functionalized synchrotron in-line phase-contrast computed tomography: a novel approach for simultaneous quantification of structural alterations and localization of bariumlabelled alveolar macrophages within mouse lung samples," Journal of synchrotron radiation, vol. 22, no. 1, pp. 0-0, 2015.

[22] M. Beltran, D. Paganin, K. Uesugi, and M. Kitchen, "2D and 3D X-ray phase retrieval of multimaterial objects using a single defocus distance," Optics express, vol. 18, no. 7, pp. 6423-6436, 2010.

[23] X. Wu, H. Liu, and A. Yan, "X-ray phase-attenuation duality and phase retrieval," Optics letters, vol. 30, no. 4, pp. 379-381, 2005.

[24] S. Mohammadi, E. Larsson, F. Alves, S. Dal Monego, S. Biffi, C. Garrovo, A. Lorenzon, G. Tromba, and C. Dullin, "Quantitative evaluation of a single-distance phase-retrieval method applied on inline phase-contrast images of a mouse lung," J Synchrotron Radiat, vol. 21, no. Pt 4, pp. 784-789, Jul. 2014.

[25] I. N. Mbawuike and H. B. Herscowitz, "MH-S, a murine alveolar macrophage cell line: morphological, cytochemical, and functional characteristics.," Journal of leukocyte biology, vol. 46, no. 2, pp. 119-127, 1989.

[26] W. Golder, M. Viermetz, and J. Gmeinwieser, "[The marking of the gastrointestinal tract in computed tomography: experiences with a barium sulfate contrast medium (Micropaque CT)]," RoFo: Fortschritte auf dem Gebiete der Rontgenstrahlen und der Nuklearmedizin, vol. 155, no. 6, pp. 562-567, 1991. 
[27] E. J. Sutton, T. D. Henning, B. J. Pichler, C. Bremer, and H. E. Daldrup-Link, "Cell tracking with optical imaging," European radiology, vol. 18, no. 10, pp. 2021-2032, 2008.

[28] P.-F. Chen, Y. Luo, W. Wang, J. Wang, W. Lai, S. Hu, K. F. Cheng, and Y. Al-Abed, "ISO-1, a macrophage migration inhibitory factor antagonist, inhibits airway remodeling in a murine model of chronic asthma," Molecular medicine, vol. 16, no. 9-10, p. 400, 2010.

[29] J. H. Bates, J. Thompson-Figueroa, L. K. Lundblad, and C. G. Irvin, "Unrestrained video-assisted plethysmography: a noninvasive method for assessment of lung mechanical function in small animals," Journal of applied physiology, vol. 104, no. 1, pp. 253-261, 2008.

[30] S. H. Bartling, W. Stiller, M. Grasruck, B. Schmidt, P. Peschke, W. Semmler, and F. Kiessling, "Retrospective Motion Gating in Small Animal CT of Mice and Rats:," Investigative Radiology, vol. 42, no. 10, pp. 704-714, Oct. 2007.

[31] A. F. Gelb, J. Licuanan, C. M. Shinar, and N. Zamel, "Unsuspected loss of lung elastic recoil in chronic persistent asthma," Chest, vol. 121, no. 3, pp. 715-721, Mar. 2002.

[32] K. Licha, P. Welker, M. Weinhart, N. Wegner, S. Kern, S. Reichert, I. Gemeinhardt, C. Weissbach, B. Ebert, and R. Haag, "Fluorescence imaging with multifunctional polyglycerol sulfates: novel polymeric near-IR probes targeting inflammation," Bioconjugate chemistry, vol. 22, no. 12, pp. 2453-2460, 2011.

[33] J. Dernedde, A. Rausch, M. Weinhart, S. Enders, R. Tauber, K. Licha, M. Schirner, U. Zügel, A. von Bonin, and R. Haag, "Dendritic polyglycerol sulfates as multivalent inhibitors of inflammation," Proceedings of the National Academy of Sciences, vol. 107, no. 46, pp. 19679-19684, 2010.

[34] Y. Mizue, S. Ghani, L. Leng, C. McDonald, P. Kong, J. Baugh, S. Lane, J. Craft, J. Nishihira, and S. Donnelly, "Role for macrophage migration inhibitory factor in asthma," Proceedings of the National Academy of Sciences of the United States of America, vol. 102, no. 40, pp. 14410-14415, 2005.

[35] X. Li and J. W. Wilson, "Increased vascularity of the bronchial mucosa in mild asthma," American Journal of Respiratory and Critical Care Medicine, vol. 156, no. 1, pp. 229-233, 1997. 


\section{APPENDIX}

\section{PUBLICATIONS IN ORDER OF THEIR APPEARANCE IN THE THESIS:}

I. T. Gureyev, S. Mohammadi, Y. Nesterets, C. Dullin, and G. Tromba, "Accuracy and precision of reconstruction of complex refractive index in near-field single-distance propagation-based phase-contrast tomography," J. Appl. Phys., vol. 114, no. 14, p. 144906, 2013.

II. S. Mohammadi, E. Larsson, F. Alves, G. Tromba, and C. Dullin, "Quantitative evaluation of a single-distance phase-retrieval method applied on in-line phase-contrast images of a mouse lung," J. Synchrotron Radiat., vol. 21, no. 4, pp. 784-789, 2014.

III. C. Dullin, E. Larsson, G. Tromba, A. Markus, F. Alves, "Phase contrast CT for quantification of structural changes in lungs of asthma mouse models of different severity," J. Synchrotron Radiat., vol. 22, pp. 1106-1111, 2015

IV. C. Dullin, E. Larsson, S. Dal Monego, S. Mohammadi, M. Krenkel, C. Garrovo, S. Biffi, A. Lorenzon, A. Markus, J. Napp, T. Salditt, A. Accardo, F. Alves, and G. Tromba, "Functionalized Synchrotron inline phase contrast CT: a novel approach for simultaneous quantification of structural alterations and localization of barium labelled alveolar macrophages within mouse lung samples," J. Synchrotron Radiat., vol. 22, pp. 143-155, 2015

V. M. Krenkel, A. Markus, M. Bartels, C. Dullin, F. Alves, T. Salditt, "Phase contrast zoomtomography reveals detailed location of macrophages in mouse lungs," Scientific reports 5, 2015.

VI. S. Biffi, S. Dal Monego, C. Dullin, C. Garrovo, B. Bosnjak, K. Licha, P. Welker, M. M. Epstein, and F. Alves, "Dendritic polyglycerolsulfate near infrared fluorescent (NIRF) dye conjugate for non-invasively monitoring of inflammation in an allergic asthma mouse model," PloS One, vol. 8, no. 2, p. e57150, 2013.

VII. M. A. Markus, C. Dullin, M. Mitkovski, E. Prieschl-Grassauer, M. M. Epstein, and F. Alves, "Non-Invasive Optical Imaging of Eosinophilia during the Course of an Experimental Allergic Airways Disease Model and in Response to Therapy," PloS One, vol. 9, no. 2, p. e90017, 2014.

VIII. A. Markus, J. Napp, T. Behnke, M. Mitkovski, C. Dullin, S. Kilfeather, U. Resch-Genger, and F. Alves, "Tracking of inhaled near-infrared nanoparticles in lungs of SKH-1 mice with allergic airway inflammation" ACS Nano, in revision 


\title{
CURRICUlum VitaE \\ Dipl. phys. Christian Dullin
}

\author{
University Medical Center Göttingen \\ Department of Diagnostic Radiology \\ Robert-Koch-Strasse 40 \\ 37075 Göttingen \\ phone: +49551398965 \\ Christian.dullin@med.uni-goettingen.de
}

Born: $\quad$ 15.11.1975 in Jena, Germany

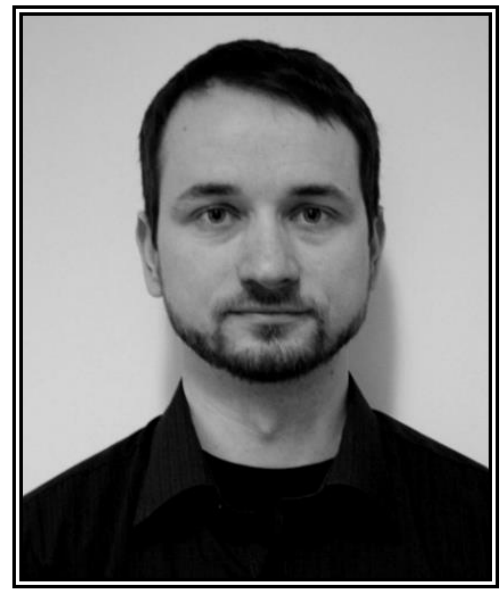

\section{Education}

04/12 - 10/15: PhD thesis on "Experimental imaging of asthma progression and therapeutic response in mouse lung models"

10/96 - 11/01: $\quad$ Technical Physics at Friedrich-Schiller-University, Jena, Germany

Degree: Physics diploma, topic: "3D skull surface reconstruction out of MRI data sets", 3di GmbH, Jena, Germany

10/82 - 07/95: $\quad$ School Education in Germany

\section{Relevant Work Experience}

11/01-11/03 Software developments for creation of patient specific skull implants, 3di GmbH, Saalbahnhofstr. 25c, Jena, Germany

02/04 - Research assistant, Diagnostic Radiology, University Medical Center Göttingen, Robert-Koch-Str. 40, 37075 Göttingen, Germany.

In charge of experimental small animal imaging (CT and optical techniques), including software development and data analysis in various applications. Contributions - in the "Molecular Imaging" - Wahlmodul of the Molecular Medicine Program Göttingen.

\section{Research Activities}

Research grants

02/13 - 02/15 Grant to foster international collaboration between Italy and Germany, DFG DU 1403/1-1

Participants in the following research grants

$08 / 09-08 / 12$

P3AGI - Public Private Partnership for Asthma Imaging and Genomics, FP7-PEOPLE2007-3-1-IAPP

$09 / 10-$

INNOVABONE - Layered hierarchical structured scaffolds with injectable self-setting bioactive gel for clinical bone tissue repair ; FP7-NMP-2010-2.2.3

01/09 - $\quad$ involvement in the project of F. Alves, DFG- SPP1190 “The Tumor Vessel - Interface” - core facility - tumor imaging

02/13 - "Extrema" COST Action 


\section{Publications}

[1] Dullin C, Larsson E, Tromba G, Markus A, Alves F. Phase contrast CT for quantification of structural changes in lungs of asthma mouse models of different severity. J. Synchrotron Radiat. 07/2015; 22(Pt 4):1106-1111.

[2] Komrakova, M, Weidemann, A, Dullin, C, Ebert, J, Tezval, M, Stuermer, KM, \& Sehmisch, S. "The Impact of Strontium Ranelate on Metaphyseal Bone Healing in Ovariectomized Rats." Calcified tissue international (2015): 1-11.

[3] Krenkel M, Markus A, Bartels M, Dullin C, Alves F, Salditt T. Phase contrast zoom-tomography reveals detailed location of macrophages in mouse lungs. Scientific Reports 5 (2015).

[4] Menke J, Kruewel T, Dullin C. Fluorochrome-labeled monoclonal antibody with characteristic M-shaped spectral peak for optical imaging: Dual-labeling versus mixture of fluorochromes. Chinese Optics Letters 01/2015; 13(5):051701-51705.

[5] Dullin C, Dal Monego S, Larsson E, Mohammadi S, Krenkel M, Garrovo C, Biffi S, Lorenzon A, Markus A, Napp J, Salditt T, Accardo A, Alves F, Tromba G. Functionalized synchrotron in-line phase-contrast computed tomography: a novel approach for simultaneous quantification of structural alterations and localization of barium-labelled alveolar macrophages within mouse lung samples. J Synchrotron Radiat. 2015 Jan;22(Pt 1):143-55.

[6] Sehmisch S, Komrakova M, Kottwitz L, Dullin C, Schmelz U, Stuermer KM, Tezval M. Effects of urocortin on spine? Results from the rat osteopenia model. Osteologie/Osteology 01/2015; 2:99-106.

[7] Reinisch A, Etchart N, Thomas D, Hofmann NA, Fruehwirth M, Sinha S, Chan CK, Senarath-Yapa K, Seo EY, Wearda T, Hartwig UF, Beham-Schmid C, Trajanoski S, Lin Q, Wagner W, Dullin C, Alves F, Andreeff M, Weissman IL, Longaker MT, Schallmoser K, Majeti R, Strunk D. Epigenetic and in-vivo comparison of diverse MSC sources reveals an endochondral signature for human hematopoietic niche formation. Blood. 2015 Jan 8;125(2):249-60.

[8] Komrakova M, Stuermer EK, Tezval M, Stuermer KM, Dullin C, Schmelz U, Doell C, Durkaya-Burchardt N, Fuerst B, Genotte T, Sehmisch S. Evaluation of twelve vibration regimes applied to improve spine properties in ovariectomized rats. Bone Rep., Dec. 2014.

[9] Gureyev T, Mayo S, Nesterets YI, Mohammadi S, Lockie D, Menk R, Arfelli F, Pavlov K, Kitchen M, and Zanconati F, Dullin C, and Tromba G, "Investigation of the imaging quality of synchrotron-based phase-contrast mammographic tomography," J. Phys. Appl. Phys., vol. 47, no. 36, p. 365401, 2014.

[10] Mohammadi S, Larsson E, Alves F, Tromba G, Dullin C. Quantitative evaluation of a single-distance phase-retrieval method applied on in-line phase-contrast images of a mouse lung. J. Synchrotron Rad. (2014).

[11] Dittmann K, Wuelling M, Uhmann A, Dullin C, Hahn H, Schweyer S, Vortkamp A, Wienands J. Inactivation of patched1 in murine chondrocytes causes spinal fusion without inflammation. Arthritis Rheumatol. 2014 Apr;66(4):831-40.

[12] Stuermer EK, Komrakova M, Sehmisch S, Tezval M, Dullin C, Schaefer N, Hallecker J, Stuermer KM. Whole body vibration during fracture healing intensifies the effects of estradiol and raloxifene in estrogen-deficient rats. Bone. 2014 Jul;64:187-94.

[13] Markus MA, Dullin C, Mitkovski M, Prieschl-Grassauer E, Epstein MM, Alves F. Non-invasive optical imaging of eosinophilia during the course of an experimental allergic airways disease model and in response to therapy. PLoS One. 2014 Feb 25;9(2):e90017.

[14] Rödig T, Reicherts P, Konietschke F, Dullin C, Hahn W, Hülsmann M. Efficacy of reciprocating and rotary NiTi instruments for retreatment of curved root canals assessed by micro-CT. International endodontic journal 47.10 (2014): 942-948.

[15] Rödig T, Kupis J, Konietschke F, Dullin C, Drebenstedt S, Hülsmann M. Comparison of hand and rotary instrumentation for removing gutta-percha from previously treated curved root canals: a microcomputed tomography study. Int Endod J. 2014 Feb;47(2):173-82.

[16] Dittmann K, Wuelling M, Uhmann A, Dullin C, Hahn H, Schweyer S, Vortkamp A, Wienands J. Inactivation of Patched 1 in chondrocytes causes spinal fusion without inflammation. Arthritis Rheum. 2013 Dec 24.

[17] Gureyev T, Mohammadi S, Nesterets Y, Dullin C, Tromba G. Accuracy and precision of reconstruction of complex refractive index in near-field single-distance propagation-based phase-contrast tomography. J. Appl. Phys. 114, 144906 (2013);

[18] Mohammadi S, Brun F, Dullin C, Dreossi D, and Tromba G, "A comparison of free software implementations of phase retrieval algorithms for propagation-based X-ray microtomographic imaging," 2013, pp. 622-626.

[19] Mathejczyk JE, Pauli J, Dullin C, Resch-Genger U, Alves F, Napp J. Errata: High-sensitivity detection of breast tumors invivo by use of a pH-sensitive near-infrared fluorescence probe. J Biomed Opt. 2013 Aug 1;18(8):89801.

[20] Rödig T, Kupis J, Konietschke F, Dullin C, Drebenstedt S, Hülsmann M. Comparison of hand and rotary instrumentation for removing gutta-percha from previously treated curved root canals: a microcomputed tomography study. Int Endod J. 2013 Apr 23. doi: 10.1111/iej.12128.

[21] Biffi S, Dal Monego S, Dullin C, Garrovo C, Bosnjak B, Licha K, Welker P, Epstein MM, Alves F. Dendritic Polyglycerolsulfate Near Infrared Fluorescent (NIRF) Dye Conjugate for Non-Invasively Monitoring of Inflammation in an Allergic Asthma Mouse Model. PLoS One. 2013;8(2):e57150.

[22] Komrakova M, Sehmisch S, Tezval M, Ammon J, Lieberwirth P, Sauerhoff C, Trautmann L, Wicke M, Dullin C, Stuermer KM, Stuermer EK. Identification of a Vibration Regime Favorable for Bone Healing and Muscle in Estrogen-Deficient Rats. Calcif Tissue Int. 2013 Feb 17.

[23] Behnke T, Mathejczyk JE, Brehm R, Würth C, Ramos Gomes F, Dullin C, Napp J, Alves F, Resch-Genger U. Target-specific nanoparticles containing a broad band emissive NIR dye for the sensitive detection and characterization of tumor development. Biomaterials. 2013 Jan;34(1):160-70. 
[24] Mathejczyk JE, Pauli J, Dullin C, Resch-Genger U, Alves F, Napp J. High-sensitivity detection of breast tumors in-vivo by use of a pH-sensitive near-infrared fluorescence probe. J Biomed Opt. 2012 Jul;17(7):076028.

[25] Rödig T, Hausdörfer T, Konietschke F, Dullin C, Hahn W, Hülsmann M. Efficacy of D-RaCe and ProTaper Universal Retreatment $\mathrm{NiTi}$ instruments and hand files in removing gutta-percha from curved root canals - a micro-computed tomography study. Int Endod J. 2012 Jun;45(6):580-9.

[26] Fricke-Zech S, Gruber RM, Dullin C, Zapf A, Kramer FJ, Kubein-Meesenburg D, Hahn W. Measurement of the midpalatal suture width. Angle Orthod. 2012 Jan;82(1):145-50. Epub 2011 Aug 3.

[27] Uhmann A, Niemann H, Lammering B, Henkel C, HeÃ̈̈ I, Rosenberger A, Dullin C, Schraepler A, Schulz-Schaeffer W, Hahn H. Calcitriol inhibits hedgehog signaling and induces vitamin d receptor signaling and differentiation in the patched mouse model of embryonal rhabdomyosarcoma. Sarcoma. 2012;2012:357040. Epub 2012 Feb 21.

[28] Rödig T, Hausdörfer T, Konietschke F, Dullin C, Hahn W, Hülsmann M. Efficacy of D-RaCe and ProTaper Universal Retreatment $\mathrm{NiTi}$ instruments and hand files in removing gutta-percha from curved root canals - a micro-computed tomography study. Int Endod J. 2012 Jun;45(6):580-9. doi: 10.1111/j.1365-2591.2012.02014.x. Epub 2012 Jan 20.

[29] Mathejczyk JE, Pauli J, Dullin C, Napp J, Tietze LF, Kessler H, Resch-Genger U, Alves F. Spectroscopically wellcharacterized RGD optical probe as a prerequisite for lifetime-gated tumor imaging. Mol Imaging. 2011 Dec;10(6):469-80. PubMed PMID: 22201538.

[30] Uhmann A, Niemann H, Lammering B, Henkel C, Fritsch A, Prüfer N, Heb I, Nitzki F, Rosenberger A, Dullin C, Schraepler A, Reifenberger PD, Schweyer S, Pietsch T, Strutz F, Schulz-Schaeffer W, Hahn H. Antitumoral effects of calcitriol in basal cell carcinomas involve inhibition of Hedgehog-signaling and induction of vitamin $D$ receptor-signaling and differentiation. Mol Cancer Ther. 2011 Aug 30.

[31] Fricke-Zech S, Gruber RM, Dullin C, Zapf A, Kramer FJ, Kubein-Meesenburg D, Hahn W. Measurement of the midpalatal suture width. Angle Orthod. 2011 Aug 3.

[32] Sausbier U*, Dullin C*, Missbach-Guentner J, Kabagema C, Flockerzie K, Kuscher GM, Stuehmer W, Neuhuber W, Ruth $\mathrm{P}$, Alves F, Sausbier M. Osteopenia due to enhanced cathepsin K release by BK channel ablation in osteoclasts. PLoS One. 2011;6(6):e21168. Epub 2011 Jun 14. (* equally contributed)

[33] Mathejczyk JE, Pauli J, Dullin C, Napp J, Tietze LF, Kessler H, Resch-Genger U, Alves F. Spectroscopically WellCharacterized RGD Optical Probe as a Prerequisite for Lifetime-Gated Tumor Imaging. Mol Imaging. 2011 Apr 27.

[34] Twarock S, Freudenberger T, Poscher E, Dai G, Jannasch K, Dullin C, Alves F, Prenzel K, Knoefel WT, Stoecklein NH, Savani RC, Homey B, Fischer JW. Inhibition of Oesophageal Squamous Cell Carcinoma Progression by in-vivo Targeting of Hyaluronan Synthesis. Mol Cancer. 2011 Mar 23;10(1):30.

[35] Hülper P, Schulz-Schaeffer W, Dullin C, Hoffmann P, Harper J, Kurtzberg L, Lonning S, Kugler W, Lakomek M, Erdlenbruch B. Tumor localization of an anti-TGF- $\beta$ antibody and its effects on gliomas. Int J Oncol. 2011 Jan;38(1):51-9.

[36] Komrakova M, Stuermer EK, Werner C, Wicke M, Kolios L, Sehmisch S, Tezval M, Daub F, Martens T, Witzenhausen P, Dullin C, Stuermer KM. Effect of human parathyroid hormone hPTH (1-34) applied at different regimes on fracture healing and muscle in ovariectomized and healthy rats. Bone. 2010 Sep;47(3):480-92.

[37] Stangl S, Gehrmann M, Dressel R, Alves F, Dullin C, Themelis G, Ntziachristos V, Staeblein E, Walch A, Winkelmann I, Multhoff G. In-vivo imaging of CT26 mouse tumours by using cmHsp70.1 monoclonal antibody. J Cell Mol Med. 2011 Apr;15(4):874-87.

[38] Grebe C, Klingebiel TM, Grau SP, Toischer K, Didié M, Jacobshagen C, Dullin C, Hasenfuss G, Seidler T. Enhanced expression of DYRK1A in cardiomyocytes inhibits acute NFAT activation but does not prevent hypertrophy in-vivo. Cardiovasc Res. 2011 Feb 20.

[39] Komrakova M, Stuermer EK, Werner C, Wicke M, Kolios L, Sehmisch S, Tezval M, Daub F, Martens T, Witzenhausen P, Dullin C, Stuermer KM. Effect of human parathyroid hormone hPTH (1-34) applied at different regimes on fracture healing and muscle in ovariectomized and healthy rats. Bone. 2010,47:480-92.

[40] Stuermer EK, Komrakova M, Werner C, Wicke M, Kolios L, Sehmisch S, Tezval M, Utesch C, Mangal O, Zimmer S, Dullin C, Stuermer KM. Musculoskeletal response to whole-body vibration during fracture healing in intact and ovariectomized rats. Calcif Tissue Int. 2010,87:168-80

[41] Hülper P, Dullin C, Kugler W, Lakomek M, Erdlenbruch B. Monitoring Proteins Using In-vivo Near-Infrared Time-Domain Optical Imaging after 2-O-Hexyldiglycerol-Mediated Transfer to the Brain. Mol Imaging Biol. 2010. [Epub ahead of print]

[42] Napp J, Dullin C, Müller F, Uhland K, Petri JB, van de Locht A, Steinmetzer T, Alves F. Time-domain in-vivo near infrared fluorescence imaging for evaluation of matriptase as a potential target for the development of novel, inhibitor-based tumor therapies. Int J Cancer. 2010,127:1958-74.

[43] Stangl S, Gehrmann M, Dressel R, Alves F, Dullin C, Themelis G, Ntziachristos V, Staeblein E, Walch A, Winkelmann I, Multhoff G. In-vivo imaging of CT26 mouse tumors by using cmHsp70.1 monoclonal antibody. J Cell Mol Med. 2010.

[44] Koenig S, Krause P, Hosseini AS, Dullin C, Rave-Fraenk M, Kimmina S, Entwistle AL, Hermann RM, Hess CF, Becker H, Christiansen $\mathrm{H}$. Noninvasive imaging of liver repopulation following hepatocyte transplantation. Cell Transplant. 2009,18:69-78.

[45] Hahn W, Fricke-Zech S, Fialka-Fricke J, Dullin C, Zapf A, Gruber R, Sennhenn-Kirchner S, Kubein-Meesenburg D, SadatKhonsari R. Imaging of the midpalatal suture in a porcine model: Flat-panel volume computed tomography compared with multislice computed tomography. Oral Surg Oral Med Oral Pathol Oral Radiol Endod. 2009,108:443-9. 
[46] Jannasch K, Dullin C, Heinlein C, Krepulat F, Wegwitz F, Deppert W, Alves F. Detection of different tumor growth kinetics in single transgenic mice with oncogene-induced mammary carcinomas by flat-panel volume computed tomography. Int $\mathrm{J}$ Cancer. 2009,125:62-70.

[47] Dullin C, Zientkowska M, Napp J, Missbach-Guentner J, Krell HW, Müller F, Grabbe E, Tietze LF, Alves F. Semiautomatic landmark-based two-dimensional-three-dimensional image fusion in living mice: correlation of near-infrared fluorescence imaging of Cy5.5-labelled antibodies with flat-panel volume computed tomography. Mol Imaging. 2009,8:2-14.

[48] Marten K, Dullin C, Machann W, Stefan Schmid J, Marco, Hermann KP, Engelke C. Comparison of flat-panel-detectorbased CT and multidetector-row CT in automated volumetry of pulmonary nodules using an anthropomorphic chest phantom. Br J Radiol. 2009,82:716-23.

[49] Alves F, Dullin C, Napp J, Missbach-Guentner J, Jannasch K, Mathejczyk J, Pardo LA, Stühmer W, Tietze LF. Concept of a selective tumour therapy and its evaluation by near-infrared fluorescence imaging and flat-panel volume computed tomography in mice. Eur J Radiol. 2009,70:286-93.

[50] Sehmisch S, Galal R, Kolios L, Tezval M, Dullin C, Zimmer S, Stuermer KM, Stuermer EK. Effects of low-magnitude, highfrequency mechanical stimulation in the rat osteopenia model. Osteoporos Int. 2009,20:1999-2008.

[51] Sehmisch S, Dullin C, Zaroban A, Tezval M, Rack T, Schmelz U, Seidlova-Wuttke D, Dunkelberg H, Wuttke W, Marten K, Stuermer KM, Stuermer EK. The use of flat panel volumetric computed tomography (fpVCT) in osteoporosis research. Acad Radiol. 2009,16:394-400.

[52] Ecke I, Petry F, Rosenberger A, Tauber S, Mönkemeyer S, Hess I, Dullin C, Kimmina S, Pirngruber J, Johnsen SA, Uhmann A, Nitzki F, Wojnowski L, Schulz-Schaeffer W, Witt O, Hahn H. Antitumor effects of a combined 5-aza2 'deoxycytidine and valproic acid treatment on rhabdomyosarcoma and medulloblastoma in Ptch mutant mice. Cancer Res. 2009,69:887-95

[53] Gruber R, Weich HA, Dullin C, Schliephake H. Ectopic bone formation after implantation of a slow release system of polylactic acid and rhBMP-2. Clin Oral Implants Res. 2009,20:24-30.

[54] Hahn W, Fricke-Zech S, Fricke J, Gruber RM, Dullin C, Zapf A, Hannig C, Kubein-Meesenburg D, Sadat-Khonsari R. Detection and size differentiation of simulated tooth root defects using flat-panel volume computerized tomography (fpVCT). Oral Surg Oral Med Oral Pathol Oral Radiol Endod. 2009,107:272-8.

[55] Hahn W, Fricke-Zech S, Fricke J, Gruber RM, Dullin C, Zapf A, Hannig C, Kubein-Meesenburg D, Sadat-Khonsari R. Detection and size differentiation of simulated tooth root defects using flat-panel volume computerized tomography (fpVCT). Oral Surg Oral Med Oral Pathol Oral Radiol Endod. 2009,107:272-8

[56] Missbach-Guentner J, Dullin C, Kimmina S, Zientkowska M, Domeyer-Missbach M, Malz C, Grabbe E, Stühmer W, Alves F. Morphologic changes of mammary carcinomas in mice over time as monitored by flat-panel detector volume computed tomography. Neoplasia. 2008,10:663-73.

[57] Majdani O, Bartling SH, Leinung M, Stöver T, Lenarz M, Dullin C, Lenarz T. A true minimally invasive approach for cochlear implantation: high accuracy in cranial base navigation through flat-panel-based volume computed tomography. 2008,29:120-3.

[58] Ecke I, Rosenberger A, Obenauer S, Dullin C, Aberger F, Kimmina S, Schweyer S, Hahn H. Cyclopamine treatment of full-blown Hh/Ptch-associated RMS partially inhibits Hh/Ptch signaling, but not tumor growth. Mol Carcinog. 2008,47:36172.

[59] Seidlová-Wuttke D, Schlumbohm C, Jarry H, Dullin C, Wuttke W. Orchidectomized (orx) marmoset (Callithrix jacchus) as a model to study the development of osteopenia/osteoporosis. Am J Primatol. 2008,70:294-300.

[60] Schliephake H, Weich HA, Dullin C, Gruber R, Frahse S. Mandibular bone repair by implantation of rhBMP-2 in a slow release carrier of polylactic acid--an experimental study in rats. Biomaterials. 2008,29:103-10.

[61] Heuser M, Hemmerlein B, Püsken M, Koskinas N, Dullin C, Gross AJ, Zöller GM, Obenauer S. Determination of renal carcinoma progression in small animals by means of flat-panel volumetric computer tomography] Urologe A. 2007,46:1710-4.

[62] Missbach-Guentner J, Dullin C, Zientkowska M, Domeyer-Missbach M, Kimmina S, Obenauer S, Kauer F, Stühmer W, Grabbe E, Vogel WF, Alves F. Flat-panel detector-based volume computed tomography: a novel 3D imaging technique to monitor osteolytic bone lesions in a mouse tumor metastasis model. Neoplasia. 2007,9:755-65.

[63] Majdani O, Bartling SH, Leinung M, Stöver T, Lenarz M, Dullin C, Lenarz T. [Image-guided minimal-invasive cochlear implantation--experiments on cadavers] Laryngorhinootologie. 2008,87:18-22.

[64] Bartling SH, Majdani O, Gupta R, Rodt T, Dullin C, Fitzgerald PF, Becker H. Large scan field, high spatial resolution flatpanel detector based volumetric CT of the whole human skull base and for maxillofacial imaging. Dentomaxillofac Radiol. 2007,36:317-27.

[65] Dullin C, Missbach-Guentner J, Vogel WF, Grabbe E, Alves F. Semi-automatic classification of skeletal morphology in genetically altered mice using flat-panel volume computed tomography. PLoS Genet. 2007,3:e118.

[66] Wessels JT, Busse AC, Mahrt J, Dullin C, Grabbe E, Mueller GA. In-vivo imaging in experimental preclinical tumor research--a review. Cytometry A. 2007,71:542-9.

[67] Ernstberger T, Heidrich G, Dullin C, Buchhorn G, Grabbe E. Preclinical evaluation by flat-panel detector-based volumetric CT versus MRI of intervertebral spacers implanted in a porcine model. Spine J. 2007,7:360-7.

[68] Obenauer S, Dullin C, Heuser M. Flat panel detector-based volumetric computed tomography (fpVCT): performance evaluation of volumetric methods by using different phantoms in comparison to 64-multislice computed tomography. Invest Radiol. 2007,42:291-6.

[69] Bartling SH, Leinung M, Graute J, Rodt T, Dullin C, Becker H, Lenarz T, Stover T, Majdani O. Increase of accuracy in intraoperative navigation through high-resolution flat-panel volume computed tomography: experimental comparison with multislice computed tomography-based navigation. Otol Neurotol. 2007,28:129-34. 
[70] Obenauer S, Dullin C, Alves F, Missbach-Guentner J, Grabbe E, Heuser M. Flat-panel-detector-based volumetric CT: performance evaluation of imaging for skeletal structures of small animals in comparison to multislice CT. Clin Imaging. 2007,31:18-22.

2006

[71] Bartling SH, Gupta R, Torkos A, Dullin C, Eckhardt G, Lenarz T, Becker H, Stöver T. Flat-panel volume computed tomography for cochlear implant electrode array examination in isolated temporal bone specimens. Otol Neurotol. 2006,27:491-8.

[72] Valencia R, Stuermer EK, Dullin C, Herrmann KP, Kluever I, Zaroban A, Sehmisch S, Funke M, Knollmann F. [Feasibility of flat-panel volumetric computed tomography (fpVCT) in experimental small animal imaging of osteoporosis - initial experience] Radiologe. 2006,46:893-9.

[73] Krneta J, Kroll J, Alves F, Prahst C, Sananbenesi F, Dullin C, Kimmina S, Phillips DJ, Augustin HG. Dissociation of angiogenesis and tumorigenesis in follistatin- and activin-expressing tumors. Cancer Res. 2006,66:5686-95.

[74] Hannig C, Krieger E, Dullin C, Merten HA, Attin T, Grabbe E, Heidrich G. Volumetry of human molars with flat panel-based volume CT in vitro. Clin Oral Investig. 2006,10:253-7.

2005

[75] Hannig C, Dullin C, Hülsmann M, Heidrich G. Three-dimensional, non-destructive visualization of vertical root fractures using flat panel volume detector computer tomography: an ex-vivo in vitro case report. Int Endod J. 2005,38:904-13.

[76] Heidrich G, Hassepass F, Dullin C, Attin T, Grabbe E, Hannig C. [Non-destructive, preclinical evaluation of root canal anatomy of human teeth with flat-panel detector volume CT (FD-VCT)] Rofo. 2005,177:1683-90.

2001

[77] Dullin C, Entwicklung einer Methode zur Segmentierung verschiedener Strukturen im Datenmaterial bildgebender Messverfahren in der Medizin (diploma thesis). Jena, Germany, Friedrich - Schiller - Universität, 2001 


\title{
Accuracy and precision of reconstruction of complex refractive index in near-field single-distance propagation-based phase-contrast tomography
}

\author{
Timur Gureyev, ${ }^{1}$ Sara Mohammadi, ${ }^{2,3}$ Yakov Nesterets, ${ }^{1}$ Christian Dullin, ${ }^{4}$ \\ and Giuliana Tromba ${ }^{2}$ \\ ${ }^{1}$ Commonwealth Scientific and Industrial Research Organisation, Australia \\ ${ }^{2}$ Elettra-Sincrotrone Trieste, Italy \\ ${ }^{3}$ The Abdus Salam ICTP, Strada Costiera 11, 34151 Trieste, Italy \\ ${ }^{4}$ University Hospital Goettingen, Germany
}

(Received 9 July 2013; accepted 19 September 2013; published online 11 October 2013)

\begin{abstract}
We investigate the quantitative accuracy and noise sensitivity of reconstruction of the 3D distribution of complex refractive index, $n(\mathbf{r})=1-\delta(\mathbf{r})+i \beta(\mathbf{r})$, in samples containing materials with different refractive indices using propagation-based phase-contrast computed tomography (PB-CT). Our present study is limited to the case of parallel-beam geometry with monochromatic synchrotron radiation, but can be readily extended to cone-beam $\mathrm{CT}$ and partially coherent polychromatic X-rays at least in the case of weakly absorbing samples. We demonstrate that, except for regions near the interfaces between distinct materials, the distribution of imaginary part of the refractive index, $\beta(\mathbf{r})$, can be accurately reconstructed from a single projection image per view angle using phase retrieval based on the so-called homogeneous version of the Transport of Intensity equation (TIE-Hom) in combination with conventional CT reconstruction. In contrast, the accuracy of reconstruction of $\delta(\mathbf{r})$ depends strongly on the choice of the "regularization" parameter in TIE-Hom. We demonstrate by means of an instructive example that for some multi-material samples, a direct application of the TIE-Hom method in PB-CT produces qualitatively incorrect results for $\delta(\mathbf{r})$, which can be rectified either by collecting additional projection images at each view angle, or by utilising suitable a priori information about the sample. As a separate observation, we also show that, in agreement with previous reports, it is possible to significantly improve signal-to-noise ratio by increasing the sample-to-detector distance in combination with TIE-Hom phase retrieval in PB-CT compared to conventional ("contact") CT, with the maximum achievable gain of the order of $0.3 \delta / \beta$. This can lead to improved image quality and/or reduction of the X-ray dose delivered to patients in medical imaging. (C) 2013 AIP Publishing LLC. [http://dx.doi.org/10.1063/1.4824491]
\end{abstract}

\section{INTRODUCTION}

After approximately 50 years of development, counting from the seminal paper by Bonse and Hart, ${ }^{1}$ phase-contrast $\mathrm{X}$-ray imaging is currently approaching a mature stage, where it can be translated into medical clinical practice. ${ }^{2,3}$ Amongst several X-ray phase-contrast imaging modalities that include crystal-based and grating-based interferometry, analyserbased imaging and others, ${ }^{3}$ the so-called in-line or propagation-based imaging (PBI) method is the simplest in its experimental implementation, as well as in its theoretical underpinnings. ${ }^{4-6}$ The method still has some well-established disadvantages, compared to alternative approaches, most notably the relatively stringent requirements to the spatial coherence of the incident X-ray beam. ${ }^{7}$ It has been recently demonstrated $^{8-11}$ that depending on the nature of the sample being imaged and the experimental layout (including the coherence properties of the source and the spatial resolution of the detector), a particular phase-contrast imaging mode may perform better than the rest, however, there is no single clear winner, in general. Therefore, in preparation to the introduction into the clinical practice and in parallel with the development of suitable new types of X-ray sources ${ }^{12-14}$ and detectors, ${ }^{15,16}$ it is important to conduct systematic investigations of the performance of each promising phasecontrast imaging method under a variety of realistic conditions. In particular, it is essential to carefully evaluate such critical parameters as spatial resolution, contrast, signal-tonoise ratio, and relevant figures-of-merits (which explicitly account for the importance of the X-ray dose delivered to the sample). While successful work of this type has been conducted recently, ${ }^{8-10,17,18}$ the need for further complementary tests still exists, e.g., in order to assess in detail the quantitative accuracy of the reconstruction of the complex refractive index or the explicit dependence of the signal-to-noise in the reconstructed images as a function of the object structure and composition, the imaging parameters and the processing algorithms in each of the relevant imaging methods.

In this work, we present the results of systematic tests of PBI in its three-dimensional form, i.e., phase-contrast propagation-based X-ray computed tomography (PB-CT). Given the requirement to the coherence of the incident $\mathrm{X}$-rays in PB-CT and the lack of readily accessible laboratory sources, which could deliver sufficient spatial coherence in combination with high photon flux, we have limited this study so far to the case of synchrotron radiation. Third-generation synchrotron sources can deliver X-ray beams with the required high coherence and high flux that allow one to conduct the 
relevant experiments (that typically involve several hundred of X-ray projection images each) within a reasonable time (a few hours, minutes, or even seconds). All the experiments considered in this paper have been carried out at SYRMEP beamline ${ }^{19}$ of Elettra synchrotron in Trieste, Italy. This beamline has been designed from the start with biomedical applications in mind, so it is well-suited for this type of imaging experiments and is also an ideal platform for pilot clinical applications. ${ }^{20} \mathrm{We}$ have performed quantitative analysis of the results of these experiments using a well-tested $\mathrm{X}$-ray image analysis software package, X-TRACT. ${ }^{21}$ This work resulted in quantitative evaluation of the accuracy of reconstruction of the complex refractive index in the multimaterial samples (where the materials have been chosen to approximate the X-ray absorption and refractive properties of biological tissues and contrast agents).

\section{MONOMORPHOUS OBJECTS}

While several theoretical methods for phase retrieval in PBI and quantitative PB-CT reconstruction have been reported, ${ }^{22-26}$ most of them require multiple $\mathrm{X}$-ray projection images to be acquired at each view angle (rotational position of the sample) in order to reconstruct the 3D distribution of the complex refractive index, $n(\mathbf{r})=1-\delta(\mathbf{r})+i \beta(\mathbf{r})$, inside the sample. At each view angle, suitable projection images can be collected at two or more different sampleto-detector distances or at different X-ray energies. ${ }^{27}$ This requirement can be easily understood as a pre-requisite for reconstruction of two independent real-valued 3D distributions, namely, those of $\delta(\mathbf{r})$ and $\beta(\mathbf{r})$, from the (real-valued) intensity measurements performed with a conventional $2 \mathrm{D}$ $\mathrm{X}$-ray area detector at different rotational positions of the sample. Known exceptions to this rule (where a single image per view angle is sufficient for an exact reconstruction) are represented by the following three cases. (1) Conventional (or "contact") CT that can be viewed as a limit case of PB$\mathrm{CT}$, in which the sample-to-detector distance is negligibly small. Here, X-ray refraction effects do not contribute to the registered images and, as a result, only the imaginary part $\beta(\mathbf{r})$ of the refractive index, which is responsible for differential absorption of X-rays in the sample, is reconstructed. ${ }^{28}$ (2) The opposite case is represented by the so-called purephase objects which exhibit negligible absorption at the $\mathrm{X}$-ray energies used in the experiment. Here, only the real part $\delta(\mathbf{r})$ of the refractive index contributes to the image contrast and thus can be reconstructed in a PB-CT experiment. ${ }^{24}$ (3) Finally, there is a class of samples characterized by a fixed proportionality relationship between the real and imaginary parts of the refractive index, $\delta(\mathbf{r}) / \beta(\mathbf{r})=\gamma$ $=$ constant $^{29-31}$ Obviously, this relationship reduces the number of unknown 3D distributions from two to just one (assuming that $\gamma$ is known a priori) and therefore a single projection per view angle is sufficient for the reconstruction of the 3D distribution of the complex refractive index $n(\mathbf{r})=1+(i-\gamma) \beta(\mathbf{r})$. Such objects are sometimes called "monomorphous," 32 they include, for example, "homogeneous" samples which consist predominantly of a single material, whose density may vary spatially. In fact, the above classes (1) and (2) can be viewed as special cases of class (3) with $\gamma=0$ and $\gamma=\infty$, respectively.

There are obvious practical advantages to using a method that requires just a single projection per view angle compared to those requiring multiple projections. Apart from the obvious reduction in the dose delivered to the sample (which can in fact be a human patient, in the case of medical applications), there are also substantial practical difficulties in applying PBI phase retrieval methods that require more than one image per view angle. In particular, due to the subtle, but complicated variation of the intensity and phase distribution in the incident $\mathrm{X}$-ray beam over time at most synchrotron beamlines, it can be difficult to properly apply the phase retrieval methods that are fundamentally based on the comparison of projection images collected at different points of time, which may be the case when using, e.g., different sample-to-detector distances. Indeed, these methods are very sensitive to low-order spatial frequencies in the images, so if the background illumination changes between different exposures even by a fraction of percent on average, the artefacts generated by these background variations after the phase retrieval tend to dominate the useful signal. ${ }^{33}$ For these and other reasons, we decided to concentrate in the present work on the application of the "monomorphous" variant of PBI phase retrieval. An obvious limitation that arises in such an approach is that of the assumption about the "monomorphicity" of the sample. Many real samples do not satisfy this assumption, although it has been demonstrated in Ref. 30 that for X-ray energies approximately between 60 and $500 \mathrm{keV}$ all biological samples consisting predominantly of chemical elements with $Z<10$, can be considered "monomorphous" for the purpose of PBI and PB-CT. Still, in general, the question needs to be addressed about the effect of the sample deviation from monomorphicity on the quantitative accuracy of phase retrieval and, ultimately, on the accuracy of reconstruction of the 3D distribution of the complex refractive index in the sample. Some publications have appeared recently, where authors have addressed this problem by different modifications of the monomorphous phase retrieval methods ${ }^{3-37}$ utilising additional a priori information about the sample. In our present analysis, we use an approach similar, in principle, to that in Ref. 34.

\section{HOMOGENEOUS TIE AND ITS USE IN PB-CT}

The most popular method currently in use for phase retrieval in PBI is based on solution of the so-called "homogeneous" Transport of Intensity Equation (TIE) proposed originally in Ref. 29. It simply inserts the "monomorphous" relationship $\delta(\mathbf{r}) / \beta(\mathbf{r})=\gamma$ into the finite-distance form ${ }^{5}$ of the original TIE, ${ }^{38}$ with the resultant equation looking as follows:

$$
I(x, y, R)=\left[1-\gamma R /(2 k) \nabla^{2}\right] I(x, y, 0),
$$

where $(x, y, z)=\mathbf{r}$ are the Cartesian spatial coordinates with $z$ axis coinciding with the optic axis (the direction of propagation of the incident $\mathrm{X}$-ray beam), $z=0$ being the location of the "object plane" and $z=R$ - the location of the detector 
(image) plane, $I(x, y, z)$ is the $\mathrm{X}$-ray intensity, $k=2 \pi / \lambda$ is the wavenumber, and $\lambda$ is the $\mathrm{X}$-ray wavelength. Equation (1) is known as "homogeneous TIE" (TIE-Hom). ${ }^{29}$ Although presented here for the case of parallel monochromatic X-ray beam, an equation similar to Eq. (1) can be derived for partially coherent polychromatic divergent X-ray beams, ${ }^{39,40}$ with the substitution of $R$ by $R^{\prime}=R_{1} R_{2} /\left(R_{1}+R_{2}\right)$ and $\gamma /(2 k)$ by $\alpha$, where $R_{1}$ and $R_{2}$ are the source-to-object and object-to-detector distances, respectively, and $\alpha$ is the position-independent proportionality coefficient between the (gradients of the) logarithm of time-averaged intensity and the generalized eikonal $\psi$ of the partially coherent beam, ${ }^{39}$ $\psi(x, y, R)=\alpha \ln I(x, y, R)$. In the case of tomographic imaging, we keep the sample fixed, together with the corresponding system of Cartesian coordinates, but rotate the optic axis and the coordinate system of the detector around the $y$ axis, so the relevant rotated coordinates are assigned subscript $\theta$ corresponding to the rotation angle $\theta^{\prime}=\theta-\pi / 2$ (Fig. 1). If in an experiment the intensity distribution $I\left(x_{\theta}, y, z_{\theta}=R\right)$ is measured in the detector plane, then Eq. (1) can be solved for the intensity distribution in the object plane ${ }^{29,30,41}$

$$
I\left(x_{\theta}, y, 0\right)=\left[1-\gamma R /(2 k) \nabla^{2}\right]^{-1} I\left(x_{\theta}, y, R\right) .
$$

At the next step, the linear integral of $\beta(\mathbf{r})$ can be obtained, assuming (as is usually done in CT) that the projection approximation is valid: ${ }^{42} \quad 2 k \int_{-\infty}^{0} \beta\left(x_{\theta}, y, z_{\theta}\right) d z_{\theta}$ $=-\ln \left[I\left(x_{\theta}, y, 0\right) / I_{i n}\right]$, where $I_{\text {in }}$ is the intensity of the (uniform) beam incident on the sample. Then, the final CT reconstruction formula in its usual Filtered Backprojection (FBP) version takes the following form: ${ }^{28}$

$$
\begin{aligned}
\beta(x, y, z)= & -\frac{1}{2 k} \int_{0}^{\pi} \int_{-\infty}^{\infty} \int_{-\infty}^{\infty} \exp \left\{-i 2 \pi\left[\xi_{\theta}(x \sin \theta+z \cos \theta)\right.\right. \\
& +\eta y]\} \mathbf{F}\left[\ln \left(I_{0} / I_{i n}\right)\right]\left(\xi_{\theta}, \eta\right)\left|\xi_{\theta}\right| d \xi_{\theta} d \eta d \theta
\end{aligned}
$$

where $I_{0}\left(x_{\theta}, y\right)=I\left(x_{\theta}, y, 0\right)$ and $\mathbf{F}$ denotes 2-dimensional Fourier transform with respect to coordinates $\left(x_{\theta}, y\right)$ in the object plane $z_{\theta}=0$, which is orthogonal to the rotated optic axis. After $\beta(\mathbf{r})$ is reconstructed, $\delta(\mathbf{r})=\gamma \beta(\mathbf{r})$ is easily obtained too. The most popular modification of the FBP formula, Eq. (3), for the case of cone-beam illumination with a small cone angle, is the well-known Feldkamp-Davis-Kress (FDK) algorithm. ${ }^{43}$ Regarding the issue of temporal coherence, we note that the CT reconstruction, Eq. (3), in principle, does not require incident $\mathrm{X}$-rays to be monochromatic. However, in the polychromatic case, the result can only be interpreted as an average over the X-ray spectrum, i.e., $\mu=\langle k \beta\rangle$, where $\mu(x, y, z)$ is the reconstructed 3D distribution of the X-ray attenuation coefficient and the angular brackets denote the spectral average. Well-known issues, such as beam hardening, have to be taken into account in this case, so the methods described in the present paper can be readily extended to polychromatic X-rays only in the case of weakly absorbing samples. ${ }^{39,44}$

If the sample is only weakly absorbing, then the following approximation can be applied: $\ln \left[1+\left(I\left(x_{\theta}, y, 0\right) / I_{\text {in }}-1\right)\right]$ $\cong I\left(x_{\theta}, y, 0\right) / I_{\text {in }}-1$. Inserting this approximation into Eq. (3), then taking Eq. (2) into account, we obtain

$$
\begin{aligned}
\beta(x, y, z)= & -\frac{1}{2 k} \int_{0}^{\pi} \int_{-\infty}^{\infty} \int_{-\infty}^{\infty} \exp \left\{-i 2 \pi\left[\xi_{\theta}(x \sin \theta+z \cos \theta)\right.\right. \\
& +\eta y]\} \mathbf{F}\left[\frac{I_{R}}{I_{i n}}-1\right]\left(\xi_{\theta}, \eta\right) \\
& \times \frac{\left|\xi_{\theta}\right|}{1+\pi \gamma \lambda R\left(\xi_{\theta}^{2}+\eta^{2}\right)} d \xi_{\theta} d \eta d \theta
\end{aligned}
$$

where $I_{R}\left(x_{\theta}, y\right)=I\left(x_{\theta}, y, R\right)$ and we have also used two wellknown properties of the Fourier transform: (i) $\mathbf{F}\left[\nabla^{2}\right]\left(\xi_{\theta}, \eta\right)$ $=-4 \pi^{2}\left(\xi_{\theta}^{2}+\eta^{2}\right)$; and (ii) $\mathbf{F}[1]\left(\xi_{\theta}, \eta\right)=\delta\left(\xi_{\theta}, \eta\right)$ (note that as the $\delta$-function is non-zero only at the origin of coordinates, it is invariant with respect to multiplication by any well-behaved function $f\left(\xi_{\theta}, \eta\right)$, such that $f(0,0)=1$ ). Equation (4) is known as the "modified Bronnikov algorithm." ${ }^{31,45}$ It provides a means for "single-step" PB-CT reconstruction of the $3 \mathrm{D}$ distribution of $\beta(\mathbf{r})$ in monomorphous objects from projection images $I\left(x_{\theta}, y, R\right)$, collected in PBI regime at the defocus distance $z_{\theta}=R$. However, as seen above, compared to the "two-step" reconstruction process represented by Eqs. (2) and (3) applied in sequence, the "single-step" formula, Eq. (4), relies on an additional assumption, namely that the object is weakly absorbing. When the latter condition is violated, the accuracy of reconstruction provided by Eq. (4) can be lower compared to that produced

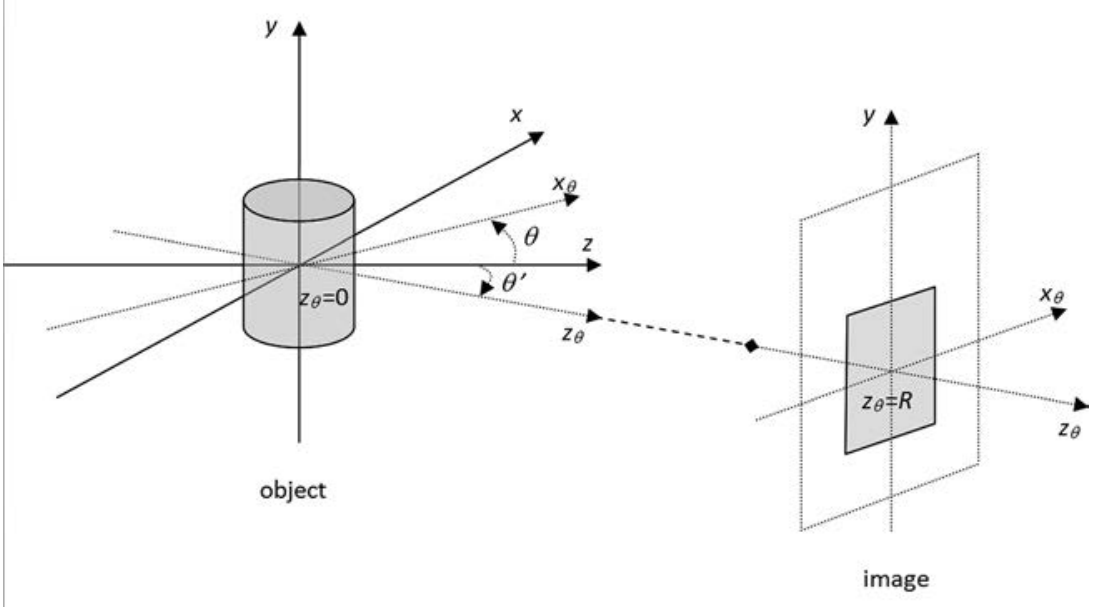

FIG. 1. PB-CT imaging geometry. 
by the combination of Eqs. (2) and (3). One possible way to circumvent this limitation is described in Ref. 46, but the reconstructed quantity in that case generally differs from both $\beta(\mathbf{r})$ and $\delta(\mathbf{r})$. Therefore, in the present work, we will use Eqs. (2) and (3) for PB-CT reconstruction. Note that an extension of Eq. (4) for the case of polychromatic conebeam CT has also been published. ${ }^{44}$

If a sample is truly monomorphous (e.g., if it consists of a single material), then the above procedures can lead to a unique and quantitatively accurate reconstruction of the complex refractive index $n(\mathbf{r})=1+(i-\gamma) \beta(\mathbf{r})$ inside the sample. If, however, the ratio $\delta(\mathbf{r}) / \beta(\mathbf{r})$ is different in different parts of the sample, this procedure inevitably produces some errors which depend on the degree of deviation of the actual distribution $\delta(\mathbf{r}) / \beta(\mathbf{r})$ from the chosen constant value $\gamma$. If the true distribution $\delta(\mathbf{r}) / \beta(\mathbf{r})$ is known a priori for a given sample, e.g., if the sample is known to consist of a small number $(M)$ of non-overlapping components, each with a constant a priori known value of $\gamma_{m}=\delta_{m} / \beta_{m}$, which may be different for different values of index $m=1,2, \ldots, M$, this information can be used to improve the accuracy of the method. One approach to effective utilization of such a priori information was proposed and successfully applied in Ref. 34. In the subsequent sections of this paper, we will use a method based on the same principle. However, first we will investigate the behaviour of the error, which the deviation of $\delta(\mathbf{r}) / \beta(\mathbf{r})$ from a constant value introduces into the reconstruction of $\delta(\mathbf{r})$ and $\beta(\mathbf{r})$ in samples that substantially violate the assumption of monomorphicity.

\section{MULTI-MATERIAL PHANTOM}

In order to investigate the accuracy of reconstruction of the complex refractive index in non-monomorphous samples, a phantom was made from a solid Polyoxymethylene (POM) cylinder with the diameter of $16.5 \mathrm{~mm}$ with 6 cylindrical holes with the diameter of $3 \mathrm{~mm}$ cored out of the POM matrix (Fig. 2) and filled with different substances, which are listed in Table I together with their densities and X-ray refractive indices at $E=31 \mathrm{keV}$ (the X-ray energy at which our PB-CT experiments were performed).

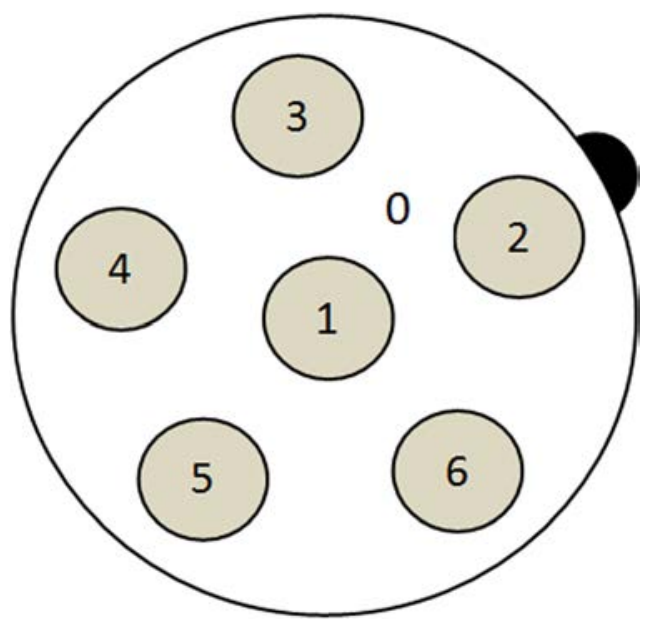

FIG. 2. Structure of the phantom used in the experiments (see Table I for materials corresponding to the indices).
TABLE I. Theoretical beta and delta values ${ }^{47}$ for materials in the phantom at $E=31 \mathrm{keV}$.

\begin{tabular}{lcccr}
\hline \hline Material & $\begin{array}{c}\text { Density } \\
\left(\mathrm{g} / \mathrm{cm}^{3}\right)\end{array}$ & $\beta \times 10^{10}$ & $\delta \times 10^{7}$ & $\delta / \beta$ \\
\hline 0. Polyoxymethylene $\left(\mathrm{CH}_{2} \mathrm{OH}\right)$ & 1.42 & 1.42 & 3.25 & 2293 \\
1. Water $\left(\mathrm{H}_{2} \mathrm{O}\right)$ & 1.00 & 1.15 & 2.40 & 2096 \\
2. Air & 0.0012 & 0.0013 & 0.0026 & 2000 \\
3. Ultravist $300\left(\mathrm{C}_{18} \mathrm{H}_{24} \mathrm{I}_{3} \mathrm{~N}_{3} \mathrm{O}_{8}\right)$ & 1.33 & 8.60 & 2.96 & 344 \\
4. Calcium chloride $1 \mathrm{M}\left(\mathrm{CaCl}_{2}\right)$ & 1.11 & 2.09 & 2.57 & 1231 \\
5. Magnesium chloride $1 \mathrm{M}\left(\mathrm{MgCl}_{2}\right)$ & 1.10 & 1.69 & 2.56 & 1512 \\
6.Glycerol $\left(\mathrm{C}_{3} \mathrm{H}_{8} \mathrm{O}_{3}\right)$ & 1.26 & 1.27 & 2.96 & 2330 \\
\hline \hline
\end{tabular}

We collected, processed and analysed CT data for the above phantom using X-rays with energy $E=31 \mathrm{keV}$ at two different sample-to-detector distances, $R=7 \mathrm{~cm}$ and $R=30 \mathrm{~cm}$. The scans were performed at the SYRMEP beamline $^{19,20}$ with the source-to-sample distance of $22.4 \mathrm{~m}$ (we included corrections for the geometrical magnification in our image processing). The detector used was a water cooled CCD camera (Photonic Science VHR, $4008 \times 2672$ full frame, pixel size $=4.5 \mu \mathrm{m}$ ) coupled to a Gadolinium Oxysulphide scintillator placed on a fiber optic taper. The CT scans were performed in $2 \times 2$ binning mode, therefore with $9 \mu \mathrm{m}$ effective pixel size. Each scan contained 1440 projections collected with $0.125^{\circ}$ angle step over $180^{\circ}$. Dark current images (without the X-ray beam) and flat field images (with X-ray beam on, but without the sample) were also collected and used for correction of the projection images of the sample in the usual manner. Figures 3(a) and 3(b) show the same slice at $y=$ constant from the conventional FBP reconstruction (using Eq. (3) with $I\left(x_{\theta}, y, R\right)$ in place of $\left.I\left(x_{\theta}, y, 0\right)\right)$ performed using projections collected at $R=7 \mathrm{~cm}$ and $R=30 \mathrm{~cm}$, respectively, as the input. ${ }^{48}$

\section{PB-CT RECONSTRUCTION OF $\beta$ USING TIE-Hom AND FBP}

The two images in Figs. 4(a) and 4(b) represent the same slice reconstructed from the CT projections collected at $R=30 \mathrm{~cm}$, but using TIE-Hom phase retrieval (i.e., using Eqs. (2) and (3) as given above) with $\gamma=2293$ (corresponding to Polyoxymethylene at $31 \mathrm{keV}$ ) and $\gamma=1048$ (corresponding to 0.5 of the $\gamma$ value for water), respectively.

One can see that, compared to the "raw" CT reconstruction in Fig. 3, the application of TIE-Hom phase retrieval: (i) noticeably improves signal-to-noise ratio in the images (see also Fig. 5); (ii) removes Fresnel fringes well (particularly with $\gamma=2293$ ); (iii) introduces some image blurring, although this blurring is not significant (as can be seen also in Fig. 5); this blurring is also lower in the case of $\gamma=1048$. Note that the blurring can become significant if different types of samples, e.g., small biological objects with high "density" of edges and interfaces, are imaged.

We then estimated the average reconstructed $\beta$ values inside each material in the phantom for different CT reconstructions, with the result presented in Table II. The uncertainties for each value given in Table II (and in relevant places in other tables below) have been calculated as the 

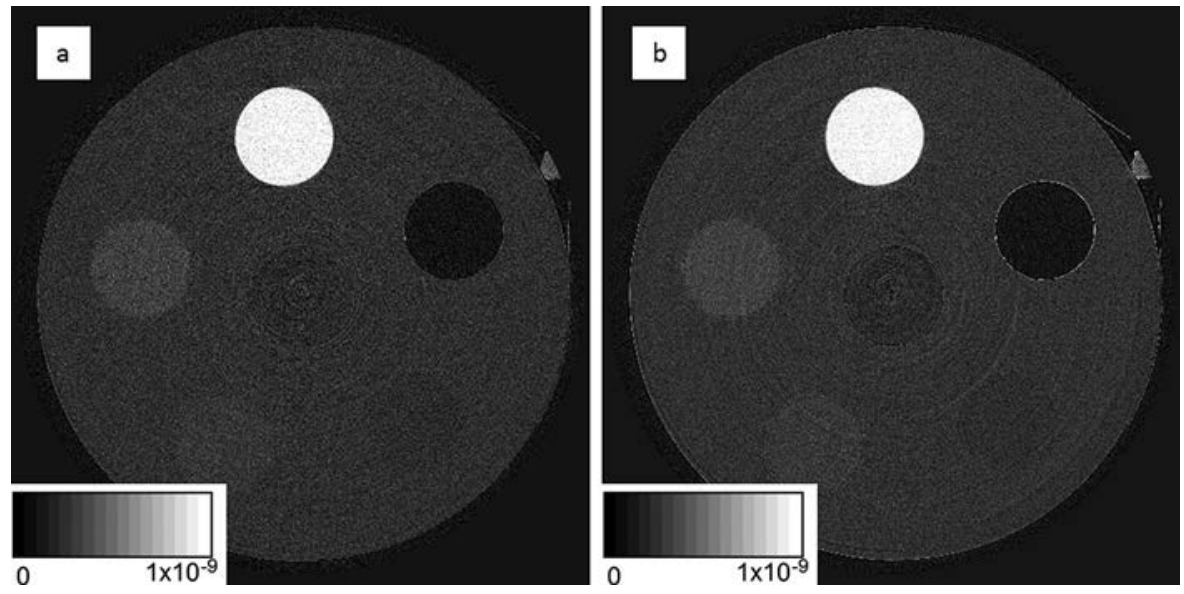

FIG. 3. FBP reconstruction (without phase retrieval) from projections collected at (a) $R=7 \mathrm{~cm}$, and (b) $R=30 \mathrm{~cm}$.
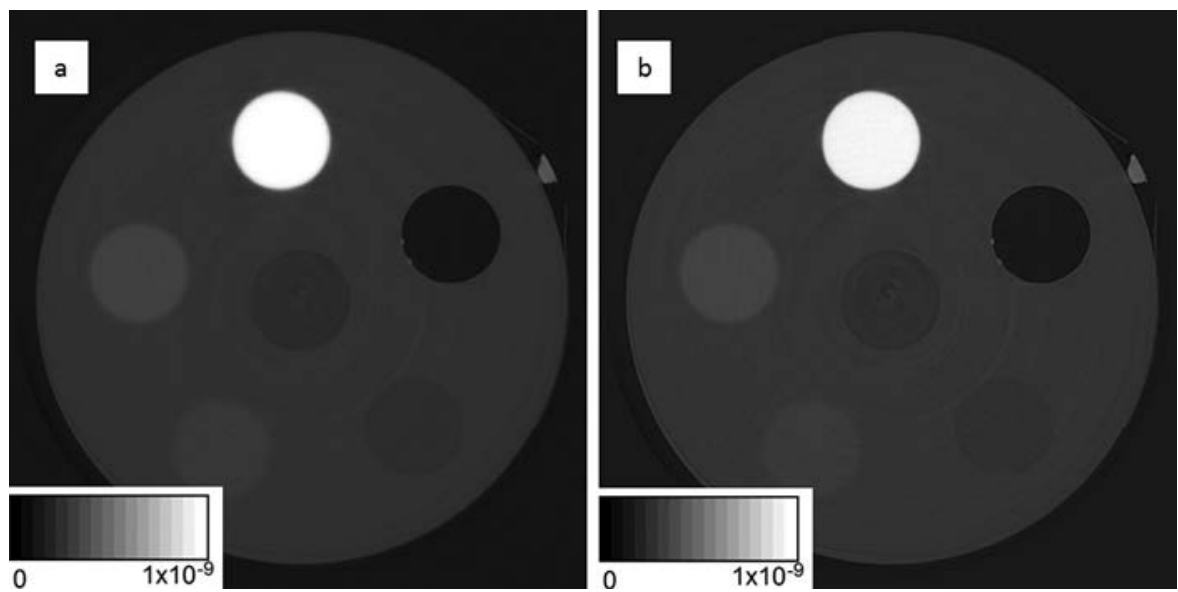

FIG. 4. FBP reconstruction from projections collected at $R=30 \mathrm{~cm}$ using TIE-Hom phase retrieval with (a) $\gamma=2293$, and (b) $\gamma=1048$.

standard deviation of the distribution of values inside the respective region (cylinder) in the reconstructed CT image (all values have been rounded to the nearest second digit after the decimal point).

Comparing the reconstructed values in Table II with the theoretical values in Table I, one can see that in all cases, the values of $\beta$ have been reconstructed with approximately the same accuracy (within $10 \%$ of the theoretical values), but much higher precision (lower standard deviation) has been achieved using the TIE-Hom phase retrieval, compared to the results obtained without phase retrieval (i.e., applying FBP reconstruction to "raw" CT projections). We have considered and checked possible sources of what appears to be a systematic $\sim 10 \%$ underestimation of the reconstructed values of $\beta$, such as the potential presence of higher-order harmonics in the incident X-ray beam, difference between the assumed and actual composition or density of the materials in the phantom, and detector performance, but have not found a definite source of this discrepancy. The analysis showed that about half of this difference $(\sim 5 \%)$ may be due to the imperfect flat-field correction due to the variation of the incident illumination during the scan. Another $5 \%$ or so are most likely to be due to some extraneous X-ray scattering contributing to the images, which led to the lower than expected (by about 5\%) apparent X-ray absorption as measured in the experimental projection images. Note that
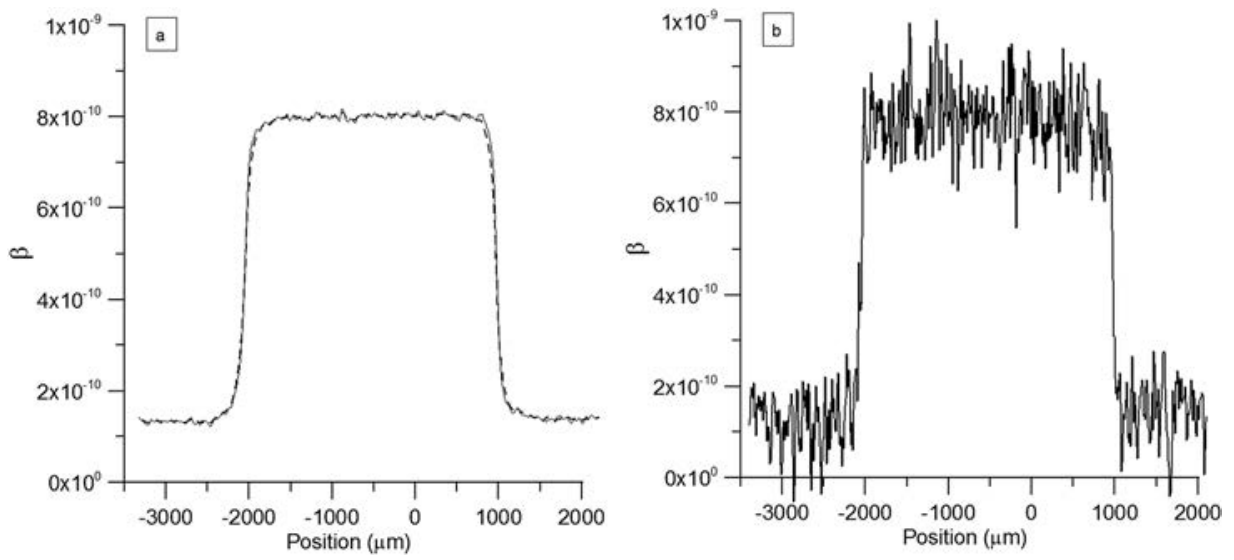

FIG. 5. Cross-sections of $\beta$ values in the Ultravist-filled cylinder in (a) Fig. 4(a) (dashed line) and Fig. 4(b) (solid line), and (b) Fig. 3(b). 
TABLE II. Values of $\beta \times 10^{10}$ for materials in the phantom reconstructed from projections at $E=31 \mathrm{keV}$ and $R=7 \mathrm{~cm}$ or $30 \mathrm{~cm}$. The values in brackets have been obtained by applying a correction factor equal to the ratio of the theoretical and reconstructed $\beta$ values for water.

\begin{tabular}{|c|c|c|c|c|}
\hline Material & $\begin{array}{l}\text { No phase retrieval } \\
\qquad(\mathrm{R}=7 \mathrm{~cm})\end{array}$ & $\begin{array}{c}\text { No phase retrieval } \\
\quad(\mathrm{R}=30 \mathrm{~cm})\end{array}$ & $\begin{array}{l}\text { TIE-Hom with } \gamma=2293 \\
\quad(\mathrm{R}=30 \mathrm{~cm})\end{array}$ & $\begin{array}{c}\text { TIE-Hom with } \gamma=1048 \\
(\mathrm{R}=30 \mathrm{~cm})\end{array}$ \\
\hline Polyoxymethylene & $\begin{array}{c}1.31 \pm 0.72 \\
(1.39 \pm 0.77)\end{array}$ & $\begin{array}{c}1.31 \pm 0.59 \\
(1.46 \pm 0.66)\end{array}$ & $\begin{array}{c}1.31 \pm 0.03 \\
(1.42 \pm 0.03)\end{array}$ & $\begin{array}{c}1.31 \pm 0.05 \\
(1.42 \pm 0.05)\end{array}$ \\
\hline Water & $\begin{array}{c}1.08 \pm 0.88 \\
(1.15 \pm 0.94)\end{array}$ & $\begin{array}{c}1.03 \pm 0.79 \\
(1.15 \pm 0.88)\end{array}$ & $\begin{array}{l}1.06 \pm 0.06 \\
(1.15 \pm 0.06)\end{array}$ & $\begin{array}{c}1.06 \pm 0.09 \\
(1.15 \pm 0.10)\end{array}$ \\
\hline Air & $\begin{array}{r}-0.00 \pm 0.67 \\
(0.00 \pm 0.71)\end{array}$ & $\begin{array}{l}-0.02 \pm 0.56 \\
(-0.02 \pm 0.63)\end{array}$ & $\begin{array}{c}-0.02 \pm 0.03 \\
(-0.02 \pm 0.03)\end{array}$ & $\begin{array}{l}-0.02 \pm 0.05 \\
(-0.02 \pm 0.05)\end{array}$ \\
\hline Ultravist 300 & $\begin{array}{c}8.03 \pm 0.90 \\
(8.55 \pm 0.96)\end{array}$ & $\begin{array}{c}8.01 \pm 0.76 \\
(8.94 \pm 0.85)\end{array}$ & $\begin{array}{l}8.02 \pm 0.04 \\
(8.69 \pm 0.04)\end{array}$ & $\begin{array}{c}8.02 \pm 0.07 \\
(8.70 \pm 0.07)\end{array}$ \\
\hline Calcium chloride & $\begin{array}{c}1.99 \pm 0.73 \\
(2.12 \pm 0.78)\end{array}$ & $\begin{array}{c}1.98 \pm 0.62 \\
(2.21 \pm 0.69)\end{array}$ & $\begin{array}{l}1.99 \pm 0.03 \\
(2.16 \pm 0.03)\end{array}$ & $\begin{array}{c}1.99 \pm 0.06 \\
(2.16 \pm 0.06)\end{array}$ \\
\hline Magnesium chloride & $\begin{array}{c}1.60 \pm 0.70 \\
(1.70 \pm 0.75)\end{array}$ & $\begin{array}{c}1.58 \pm 0.58 \\
(1.76 \pm 0.65)\end{array}$ & $\begin{array}{l}1.58 \pm 0.03 \\
(1.71 \pm 0.03)\end{array}$ & $\begin{array}{c}1.58 \pm 0.05 \\
(1.71 \pm 0.05)\end{array}$ \\
\hline Glycerol & $\begin{array}{l}1.18 \pm 0.68 \\
(1.26 \pm 0.77)\end{array}$ & $\begin{array}{c}1.14 \pm 0.58 \\
(1.28 \pm 0.65)\end{array}$ & $\begin{array}{c}1.14 \pm 0.03 \\
(1.24 \pm 0.03)\end{array}$ & $\begin{array}{c}1.14 \pm 0.05 \\
(1.24 \pm 0.05)\end{array}$ \\
\hline
\end{tabular}

although the sources of the observed difference between the theoretical and experimental values of $\beta$ may be worth further investigation in subsequent studies at SYRMEP beamline, it is important to note that depending on the published source, where the theoretical values of $\beta$ are obtained from, they can also differ by up to $5 \%$ (see, e.g., the NIST X-ray Form Factor, Attenuation and Scattering Tables at http://www.nist.gov/pml/data/ffast/index.cfm, Sergey Stepanov's X-ray Server at Argonne National Laboratory, http://sergey.gmca.aps.anl.gov, Henke tables at Berkeley Lab, http://henke.lbl.gov/optical_constants, or the data based on Ref. 47 at our website, http://www.ts-imaging.net/ Services/Simple/ICUtilAbsorb.aspx).

We then further quantified the precision of the reconstruction by calculating the signal-to-noise ratio (SNR) in the usual fashion

$$
\mathrm{SNR}=\frac{\left|\left\langle\beta_{\text {feature }}\right\rangle-\left\langle\beta_{\text {background }}\right\rangle\right|}{\left(\sigma_{\text {feature }}^{2}+\sigma_{\text {background }}^{2}\right)^{\frac{1}{2}}},
$$

where "features" in the phantom correspond to the small cylinders filled with various substances and the "background" corresponds to the enclosing POM cylinder, angular brackets denote the average values, and $\sigma^{2}$ stands for the variance of the reconstructed $\beta$ values inside the corresponding cylinders.

Table III clearly shows that, consistent with the visual impression from Figs. 3-5, the SNR of the reconstructed $\beta$ values in PB-CT images obtained with the help of TIE-Hom phase retrieval is considerably higher when compared to that in the CT images obtained without the phase retrieval, which is consistent with previous reports. ${ }^{34-37,49}$

The SNR numbers in Table III are also consistent with the theoretical formulae obtained in Ref. 50

$$
\frac{\mathrm{SNR}_{\mathrm{PB}-\mathrm{CT}}}{\mathrm{SNR}_{\text {contact-CT }}} \cong \frac{c_{1} \gamma}{N_{\min }^{F}}\left[\ln \left(\frac{c_{2} \gamma}{N_{\text {min }}^{F}}\right)\right]^{-1 / 2},
$$

where $\mathrm{SNR}_{\mathrm{PB}-\mathrm{CT}}$ and $\mathrm{SNR}_{\text {contact-CT }}$ denote, respectively, the signal-to-noise ratio in CT images obtained in PB-CT with the use of TIE-Hom phase retrieval and in conventional (contact) CT images, $N_{\text {min }}^{F}=h^{2} /(\lambda R)$ is the "minimal Fresnel number" associated with the CT projections, $h$ is the detector pixel size, $c_{1}$ and $c_{2}$ are constants which depend on the ratio of the width of detector's point-spread function (PSF) to its pixel $\operatorname{size}^{50}$ (Equation (6) is actually a simplified expression obtained under the assumption $\left(\gamma / N_{\min }^{F}\right) \gg 1$ from a more general equation found in Ref. 50). In a typical case of the detector PSF being 2 to 3 pixels wide, $c_{1} \cong 0.079$ and $c_{2} \cong 0.016$. In our experiment, we had: $\lambda=0.04 \mathrm{~nm}$, $h=9 \mu \mathrm{m}, R=30 \mathrm{~cm}$, and hence $N_{\min }^{F}=6.75$. Therefore, for the two values of $\gamma$ that we used for the TIE-Hom reconstruction, we obtain from Eq. (6), the theoretical gain factors of 20.5 and 12.7 for $\gamma=2293$ and $\gamma=1048$, respectively. On the other hand, calculating the ratio $\mathrm{SNR}_{\mathrm{PB}-\mathrm{CT}} / \mathrm{SNR}_{\text {contact-CT }}$ directly from the reconstructed experimental images for different materials in the phantom (see Table IV), we obtain the average values of 18.45 and 10.92 (or 19.48 and 11.37, if we exclude water from the estimations) for the same two values of $\gamma$, which agree reasonably well with the above estimation obtained from Eq. (6).

TABLE III. SNR for materials in the phantom reconstructed from projections at $E=31 \mathrm{keV}$.

\begin{tabular}{lcccc}
\hline \hline Material & $\begin{array}{c}\text { No phase } \\
\text { retrieval } \\
(\mathrm{R}=7 \mathrm{~cm})\end{array}$ & $\begin{array}{c}\text { No phase } \\
\text { retrieval } \\
(\mathrm{R}=30 \mathrm{~cm})\end{array}$ & $\begin{array}{c}\text { TIE-Hom } \\
\text { with } \\
\gamma=2293\end{array}$ & $\begin{array}{c}\text { TIE-Hom } \\
\text { with } \\
\gamma=1048\end{array}$ \\
\hline Polyoxymethylene & N/A & N/A & N/A & N/A \\
Water & 0.2 & 0.3 & 3.7 & 2.4 \\
Air & 1.3 & 1.6 & 31.4 & 18.8 \\
Ultravist 300 & 5.8 & 7.1 & 134.8 & 78.0 \\
Calcium chloride & 0.7 & 0.8 & 16.0 & 8.7 \\
Magnesium chloride & 0.3 & 0.3 & 6.4 & 3.8 \\
Glycerol & 0.1 & 0.2 & 4.0 & 2.4 \\
\hline \hline
\end{tabular}


TABLE IV. SNR $\mathrm{PB}_{\mathrm{PCT}} / \mathrm{SNR}_{\text {contact-CT }}$ ratio for materials in the phantom reconstructed from projections at $E=31 \mathrm{keV}$ and $R=30 \mathrm{~cm}$.

\begin{tabular}{lcr}
\hline \hline Material & $\gamma=2293$ & $\gamma=1048$ \\
\hline Polyoxymethylene & N/A & N/A \\
Water & 13.3 & 8.7 \\
Air & 19.1 & 11.5 \\
Ultravist 300 & 19.4 & 11.2 \\
Calcium chloride & 20.6 & 11.2 \\
Magnesium chloride & 19.3 & 11.6 \\
Glycerol & 19.1 & 11.4 \\
\hline \hline
\end{tabular}

Note that according to Eq. (6), it should be possible to increase the SNR in the phase-retrieved PB-CT images even further by choosing a larger value for $\gamma$ in TIE-Hom phase retrieval. However, this would lead to increased blurring of boundaries and interfaces in the image. Furthermore, one could argue that the SNR in conventional CT images can be increased simply by spatial filtering, instead of the TIE-Hom phase retrieval (after all, mathematically, TIE-Hom phase retrieval represents a form of Lorentzian low-pass filtering). However, any low-pass spatial filtering of either projections or reconstructed CT images will, in general, lead to loss of spatial resolution. In contrast to that, the combination of free-space propagation followed by phase retrieval does not result in a loss of spatial resolution in the phase-retrieved images, as long as the validity conditions of the relevant phase retrieval method are satisfied. Indeed, the spatial filtering associated with TIE-Hom phase retrieval can be viewed as precise compensation of the Fresnel fringes appearing in the images due to free-space propagation. ${ }^{51}$ Regarding the spatial blurring in the CT images obtained with the help of TIE-Hom in the present case, we note that the blurring was absent at the boundaries of those cylinders, whose $\delta / \beta$ ratio was equal or close to $\gamma$ used in the TIE-Hom based phase retrieval. Thus, in the examples above, the edge of the POM cylinder did not display any additional blurring after the application of TIE-Hom with $\gamma=2293$, while the most blurred edge was that of the Ultravist-filled cylinder shown in Fig. 5 (as this cylinder had the lowest $\gamma$ ratio, see the last column in Table I). The situation would be different if we use TIE-Hom with $\gamma=344$ (the value that corresponds to Ultravist): in that case, the boundaries of the Ultravist-filled cylinder would not be blurred, but the other boundaries in the phantom would retain some "remnants" of the Fresnel diffraction fringes, i.e., the phase-contrast effects at most of the boundaries would be "under-compensated." 34,35

Thus, there is no "silver bullet": it is impossible to properly compensate for the effects of Fresnel diffraction at boundaries of materials with different $\delta / \beta$ ratios using the TIE-Hom method with a constant $\gamma$. More generally, a tradeoff always exists in PB-CT imaging between the SNR and the spatial resolution in the reconstructed images (compare with Refs. 17 and 18). Unfortunately, the optimum imaging conditions may depend on the (a priori unknown) internal geometry and composition of the sample. Furthermore, the optimum imaging conditions can be different for different parts of the same sample. Still, some rough "rule of thumb" type estimate for the optimal imaging conditions (likely to maximize the SNR in the phase-retrieved PB-CT images) can be deduced from Eq. (6). One can see that the ratio $\mathrm{SNR}_{\mathrm{PB}-\mathrm{CT}} / \mathrm{SNR}_{\text {contact-CT }}$ increases, when $N_{\min }^{F}$ decreases, i.e., when one or more of the following happens: the propagation distance increases or the detector pixel size decreases. The dependence on the wavelength (energy) is more complicated due to the fact that both $N_{\min }^{F}$ and $\delta / \beta$ ratio depend on the $\mathrm{X}$-ray energy. As a result, the ratio $\mathrm{SNR}_{\mathrm{PB}-\mathrm{CT}} / \mathrm{SNR}_{\text {contact-CT }}$ decreases both at low and high energies, reaching its maximum at a particular energy which depends on the material composition of the sample (in particular, for breast tissue, this maximum is achieved at approximately $20 \mathrm{keV}$ ). Note however that Eq. (6) is based on the TIE theory, which is valid only when $N_{\min }^{F}>1 .^{38}$ If has been shown previously ${ }^{18}$ that in the region $N_{\min }^{F}<1$, the phase contrast in PBI ceases to increase linearly with $1 / N_{\text {min }}^{F}$, and instead asymptotically approaches a constant maximum value. A similar result has been obtained in Ref. 50 for the behaviour of the ratio $\mathrm{SNR}_{\mathrm{PB}-\mathrm{CT}} / \mathrm{SNR}_{\text {contact-CT }}$ at small values of $N_{\min }^{F}$ in PB-CT imaging. Therefore, we can infer that $\mathrm{SNR}_{\mathrm{PB}-\mathrm{CT}} / \mathrm{SNR}_{\text {contact-CT }}$ reaches its maximum approximately when $N_{\min }^{F} \sim 1$, and the maximum "gain" $\mathrm{SNR}_{\mathrm{PB}-\mathrm{CT}} / \mathrm{SNR}_{\text {contact-CT }}$ is approximately equal to $0.3 \delta / \beta$. This estimation has been obtained in Ref. 50 semi-empirically as a result of numerical simulations and analysis of the behaviour of the more general expressions for both cases, $N_{\text {min }}^{F}>1$ and $N_{\text {min }}^{F}<1$.

\section{3D RECONSTRUCTION OF $\boldsymbol{\delta}$}

As mentioned in Sec. I, Beltran et al. ${ }^{34,35}$ have proposed a PB-CT method utilising a priori known information about the varying $\delta / \beta$ ratio across different regions of the sample. This approach allows one to overcome the limitations of the conventional TIE-Hom method, provided that regions in the sample with different $\gamma$ values are suitably separated. Here, we apply a different, but closely related, procedure for reconstruction of the 3D distribution of $\delta(\mathbf{r})$ in a multi-material phantom. We have calculated the distribution of $\delta(\mathbf{r})$ in our phantom by segmenting the reconstructed distribution of $\beta$ obtained using TIE-Hom with $\gamma=2293$ followed by FBP, from projections collected at $R=30 \mathrm{~cm}$, and multiplying different segmented areas of the reconstructed $\beta$ distribution by the corresponding local values of $\delta / \beta$ shown in the last column of Table I. Table $\mathrm{V}$ below presents the analysis of the distribution of $\delta(\mathbf{r})$ obtained by this method.

One can see that (i) the reconstructed values of $\delta$ are generally about $10 \%$ lower when compared to the theoretical values, which is a direct consequence of the same relationship between the reconstructed and theoretical values of $\beta$ in Table II; (ii) the SNR values of the reconstructed $\delta$ values are of the same order as those for $\beta$ in Table III, except for Ultravist, where the SNR for $\delta$ is about 30 times lower than that for $\beta$. The latter deviation can be explained by the fact that the difference between the X-ray absorption of Ultravist and that of the "background material" (Polyoxymethylene) is much larger than the difference between the refractive properties of the same materials. 
TABLE V. Values of $\delta \times 10^{7}$ obtained from the distribution of $\beta(\mathbf{r})$ reconstructed using TIE-Hom with $\gamma=2293$ followed by FBP, from projections collected at $R_{2}=30 \mathrm{~cm}$, subsequent segmentation and multiplication by suitable $\delta / \beta$ in each cylinder. The values in brackets have been obtained by applying a correction factor equal to the ratio of the theoretical and reconstructed $\delta$ values for water. SNR was calculated as for $\beta$ above.

\begin{tabular}{lccc}
\hline \hline Material & Theoretical & Reconstructed & SNR \\
\hline Polyoxymethylene & 3.25 & $3.02 \pm 0.07$ & N/A \\
& & $(3.28 \pm 0.08)$ & \\
Water & 2.40 & $2.21 \pm 0.12$ & 4.16 \\
& & $(2.40 \pm 0.13)$ & \\
Air & 0.0026 & $-0.04 \pm 0.07$ & 20.95 \\
& & $(-0.04 \pm 0.08)$ & \\
Ultravist 300 & 2.96 & $2.64 \pm 0.02$ & 5.22 \\
& & $(2.87 \pm 0.02)$ & \\
Calcium chloride & 2.57 & $2.45 \pm 0.04$ & 5.05 \\
& & $(2.66 \pm 0.04)$ & \\
Magnesium chloride & 2.56 & $2.39 \pm 0.05$ & 5.23 \\
& & $(2.60 \pm 0.05)$ & \\
Glycerol & 2.96 & $2.66 \pm 0.08$ & 2.39 \\
& & $(2.89 \pm 0.09)$ & \\
\hline \hline
\end{tabular}

We have seen above (Table II) that the use of TIE-Hom in combination with FBP reconstruction in $\mathrm{PB}-\mathrm{CT}$ led to quantitatively accurate reconstruction of the $3 \mathrm{D}$ distribution of the imaginary part $\beta(\mathbf{r})$ of the complex refractive index in a multi-material sample, regardless of the chosen constant value of $\gamma$ used at the TIE-Hom phase retrieval step. The only obvious consequence of the variation of $\delta(\mathbf{r}) / \beta(\mathbf{r})$ in the sample, in this case, is the under- or over-compensation of Fresnel fringes near the interfaces. In stark contrast, the reconstruction of $3 \mathrm{D}$ distribution of the real part $\delta(\mathbf{r})$ of the complex refractive index in a multi-material sample by using TIE-Hom with a constant value of $\gamma$, in combination with FBP, can be qualitatively inaccurate as illustrated by Fig. 6, and particularly Fig. 7. The latter figure shows line profiles of the reconstructed $\delta(\mathbf{r})$ values in Figs. 6(a) and 6(b) taken across the cylinders filled with water and $\mathrm{CaCl}_{2}$ solution. One can see that the two cylinders produced two "dips" in Fig. 7(a), while in Fig. 7(b), the water-filled cylinder still produced a "dip," but the $\mathrm{CaCl}_{2}$-filled cylinder produced a "peak." The correct qualitative behaviour is, of course, that in Fig. 7(a), as can be seen from the theoretical values of $\delta$ given in the first column of Table $\mathrm{V}$. The qualitatively wrong behaviour of the distribution of $\delta(\mathbf{r})$ obtained by TIE-Hom with constant $\gamma$ is caused here by the fact that $\beta$ (water) $<\beta$ (POM), but $\beta\left(\mathrm{CaCl}_{2}\right)>\beta(\mathrm{POM})$, while both $\delta$ (water $)<\delta(\mathrm{POM})$ and $\delta\left(\mathrm{CaCl}_{2}\right)<\delta(\mathrm{POM})$ (see Table I). In other words, the relative ratio $[\delta($ water $)-\delta(\mathrm{POM})] /$ $[\beta$ (water) $-\beta(\mathrm{POM})]$ is positive, while the ratio $\left[\delta\left(\mathrm{CaCl}_{2}\right)\right.$ $\delta(\mathrm{POM})] /\left[\beta\left(\mathrm{CaCl}_{2}\right)-\beta(\mathrm{POM})\right]$ is negative. This phenomenon (change of sign of the relative delta-to-beta ratio across the sample) is impossible to take into account in the TIE-Hom phase retrieval, which assumes a constant $\delta / \beta$ ratio across
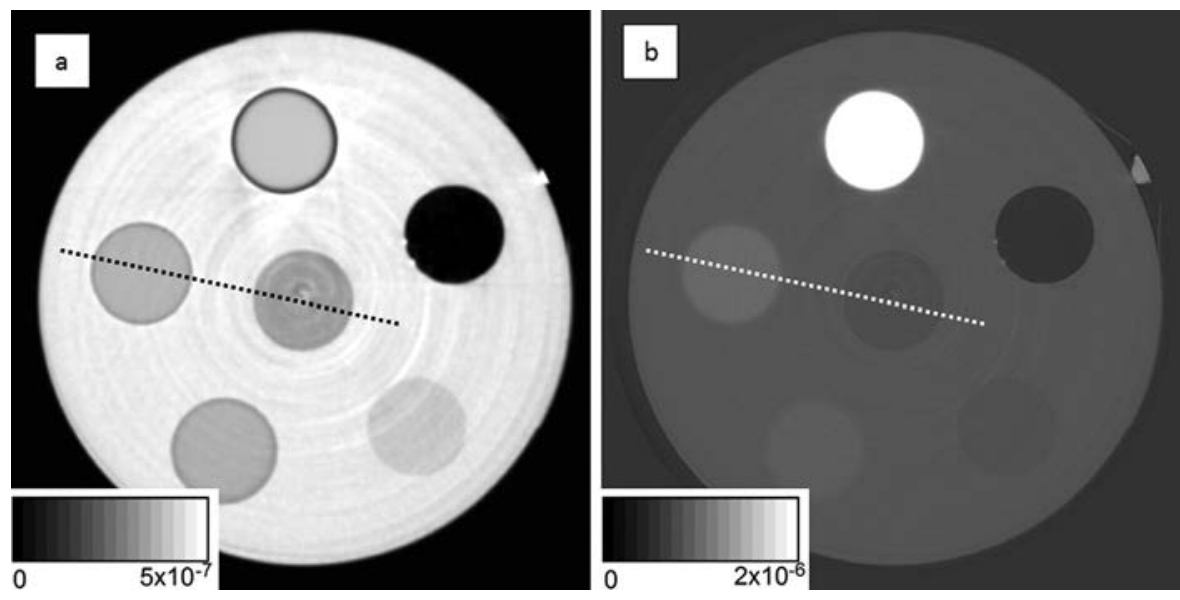

FIG. 6. The reconstructed distribution of $\delta$ obtained by (a) segmenting the reconstructed $\beta$ distribution from Fig. 4(a) and multiplying values in each cylinder by the corresponding value of $\gamma_{i}$ from the last column of Table I, and (b) multiplying the reconstructed $\beta$ distribution from Fig. 4(a) by a constant value $\gamma=2293$.
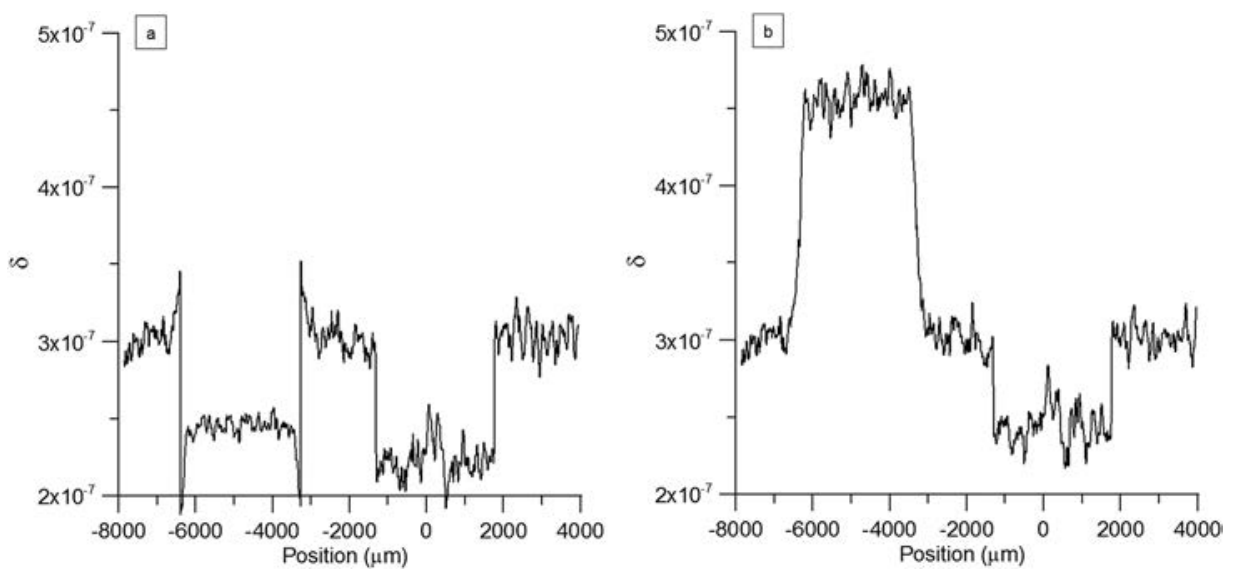

FIG. 7. Sections of the reconstructed distribution of $\delta(\mathbf{r})$ across the cylinders filled with water and $\mathrm{CaCl}_{2}$ solution along the line shown in Figs. 6(a) and $6(b)$. 
the whole sample, leading to qualitative inconsistencies in the reconstruction of $\delta$ as shown in Fig. 7. Note that in the method proposed by Beltran et al. ${ }^{34}$ a negative relative $\delta / \beta$ ratio can cause instabilities in the phase reconstruction (see Eq. (9) in Ref. 34). On the other hand, the main shortcoming of the method used for 3D reconstruction of $\delta(\mathbf{r})$ in the present work can be seen in the form of the dark "halo" around the Ultravist-filled cylinder in Fig. 6(a). This defect is caused by the blurring of the edge of the Ultravist-filled cylinder in the 3D reconstruction of $\beta(\mathbf{r})$ as seen in Figs. 4(a) and 5(a).

\section{CONCLUSIONS}

We have presented quantitative analysis of the reconstruction of complex refractive index in a sample containing materials with substantially different X-ray absorption and refraction properties, using variants of TIE-Hom phase retrieval method in combination with conventional CT reconstruction. We have demonstrated that the 3D distribution of the imaginary part, $\beta(\mathbf{r})$, of the complex refractive index can, in general, be reconstructed with high accuracy by this method regardless of the choice of the regularization parameter $\gamma$ in TIE-Hom. The only noticeable effect of the variation of $\delta(\mathbf{r}) / \beta(\mathbf{r})$ ratio across the sample is the incomplete removal of Fresnel diffraction fringes or the blurring of the interfaces, in the areas of the sample, where the actual local $\delta / \beta$ ratio differs from the chosen value of $\gamma$. Additionally, when compared to the CT reconstruction from "contact" projections (collected at small sampleto-detector distances), the use of large sample-to-detector distances in combination with TIE-Hom phase retrieval, leads to significant increases in the precision of reconstruction of the 3D distribution of $\beta(\mathbf{r})$ across the sample. In other words, in agreement with previously reported results, the SNR in the distribution of $\beta(\mathbf{r})$ reconstructed this way, is considerably larger when compared to "contact" CT or PB-CT without phase retrieval. On the other hand, the same method (i.e., the TIE-Hom phase retrieval followed by conventional $\mathrm{CT}$ reconstruction) generally cannot guarantee even a qualitative accuracy in the reconstruction of the real part, $\delta(\mathbf{r})$, of the complex refractive index. Indeed, if a constant $\delta / \beta$ ratio is assumed, as required in the conventional TIE-Hom method, an actual variation of $\delta(\mathbf{r}) / \beta(\mathbf{r})$ ratio across the sample can cause the reconstructed $\delta(\mathbf{r})$ distribution to qualitatively differ from the real one. In other words, for two regions, $A$ and $B$, in a sample, with $\delta_{\text {true }}(A)>\delta_{\text {true }}(B)$, the reconstructed values of $\delta$ can satisfy the opposite relationship, $\delta_{\text {reconst }}(A)$ $<\delta_{\text {reconst }}(B)$. Such errors can be avoided if additional a priori information about the sample is available, e.g., in the form of knowledge about the actual distribution of $\delta(\mathbf{r}) / \beta(\mathbf{r})$ in the sample. In that case, for some classes of samples, quantitatively accurate reconstruction of $\delta(\mathbf{r})$ can also be obtained with a similar accuracy and precision achievable for $\beta(\mathbf{r})$. We presented one example of such "modified TIE-Hom plus FBP" reconstruction, which is similar, in principle, to that in Ref. 34 , but may produce better results in certain cases.
Overall, the results presented in this paper point to potential benefits that the use of larger sample-to-detector distances in combination with TIE-Hom phase retrieval can deliver. The demonstrated significant increases in the SNR in the reconstructed 3D images, which is achieved primarily due to the contribution from differences in the refractive properties across the sample in addition to the differential absorption, can lead to substantial decreases in the dose delivered to the sample and/or to improvements in the quality of the reconstructed images. The increase in the sampleto-detector distance also reduces the contribution of X-ray scatter to the projection images, which is likely to further improve the SNR in the final reconstructed images. These favourable properties point to exciting opportunities for the use of this type of methods in clinical radiography and other application areas. ${ }^{2,3,52,53}$

${ }^{1}$ U. Bonse and M. Hart, Appl. Phys. Lett. 6(8), 155-156 (1965).

${ }^{2}$ A. Olivo and I. Robinson, "Taking X-ray phase contrast imaging into mainstream applications," Philos. Trans. R. Soc. London, Ser. A (unpublished), available at http://royalsociety.org/events/2013/x-ray-phase-contrast/.

${ }^{3}$ A. Bravin, P. Coan, and P. Suortti, Phys. Med. Biol. 58(1), R1-R35 (2013).

${ }^{4}$ A. Snigirev, I. Snigireva, V. Kohn, S. Kuznetsov, and I. Schelokov, Rev. Sci. Instrum. 66(12), 5486-5492 (1995).

${ }^{5}$ S. W. Wilkins, T. E. Gureyev, D. Gao, A. Pogany, and A. W. Stevenson, Nature 384(6607), 335-338 (1996).

${ }^{6}$ P. Cloetens, R. Barrett, J. Baruchel, J. P. Guigay, and M. Schlenker, J. Phys. D: Appl. Phys. 29(1), 133-146 (1996).

${ }^{7}$ K. A. Nugent, Adv. Phys. 59(1), 1-99 (2010).

${ }^{8}$ M. Langer, P. Cloetens, J.-P. Guigay, and F. Peyrin, Med. Phys. 35(10), 4556-4566 (2008).

${ }^{9}$ P. C. Diemoz, A. Bravin, and P. Coan, Opt. Express 20(3), 2789-2805 (2012).

${ }^{10}$ P. C. Diemoz, A. Bravin, M. Langer, and P. Coan, Opt. Express 20(25), 27670-27690 (2012).

${ }^{11}$ A. Burvall, U. Lundstrom, P. A. Takman, D. H. Larsson, and H. M. Hertz, Opt. Express 19(11), 10359-10376 (2011).

${ }^{12}$ D. H. Larsson, P. A. Takman, U. Lundstrom, A. Burvall, and H. M. Hertz, Rev. Sci. Instrum. 82(12), 123701 (2011).

${ }^{13}$ S. Fourmaux, S. Corde, K. T. Phuoc, P. Lassonde, G. Lebrun, S. Payeur, F. Martin, S. Sebban, V. Malka, A. Rousse, and J. C. Kieffer, Opt. Lett. 36(13), 2426-2428 (2011).

${ }^{14}$ M. Endrizzi, T. E. Gureyev, P. Delogu, P. Oliva, B. Golosio, M. Carpinelli, I. Pogorelsky, V. Yakimenko, and U. Bottigli, Opt. Express 19(3), 2748-2753 (2011).

${ }^{15}$ F. Krejci, J. Jakubek, and M. Kroupa, Nucl. Instrum. Methods Phys. Res. A 633, S181-S184 (2011).

${ }^{16}$ H. Jiang, Q. Zhao, L. E. Antonuk, Y. El-Mohri, and T. Gupta, Phys. Med. Biol. 58(3), 703-714 (2013).

${ }^{17}$ Y. I. Nesterets, S. W. Wilkins, T. E. Gureyev, A. Pogany, and A. W. Stevenson, Rev. Sci. Instrum. 76(9), 093706 (2005).

${ }^{18}$ T. E. Gureyev, Y. I. Nesterets, A. W. Stevenson, P. R. Miller, A. Pogany, and S. W. Wilkins, Opt. Express 16(5), 3223-3241 (2008).

${ }^{19}$ A. Abrami, F. Arfelli, R. C. Barroso, A. Bergamaschi, F. Billè, P. Bregant, F. Brizzi, K. Casarin, E. Castelli, V. Chenda, L. D. Palma, D. Dreossi, C. Fava, R. Longo, L. Mancini, R. H. Menk, F. Montanari, A. Olivo, S. Pani, A. Pillon, E. Quai, S. R. Kaiser, L. Rigon, T. Rokvic, M. Tonutti, G. Tromba, A. Vascotto, C. Venanzi, F. Zanconati, A. Zanetti, and F. Zanini, Nucl. Instrum. Methods Phys. Res. A 548(1-2), 221-227 (2005).

${ }^{20}$ E. Castelli, M. Tonutti, F. Arfelli, R. Longo, E. Quaia, L. Rigon, D. Sanabor, F. Zanconati, D. Dreossi, A. Abrami, E. Quai, P. Bregant, K. Casarin, V. Chenda, R. H. Menk, T. Rokvic, A. Vascotto, G. Tromba, and M. A. Cova, Radiology 259(3), 684-694 (2011).

${ }^{21}$ T. E. Gureyev, Y. Nesterets, D. Ternovski, D. Thompson, S. W. Wilkins, A. W. Stevenson, A. Sakellariou, and J. A. Taylor, Proc. SPIE 8141, 81410B (2011).

${ }^{22}$ K. A. Nugent, T. E. Gureyev, D. F. Cookson, D. Paganin, and Z. Barnea, Phys. Rev. Lett. 77(14), 2961-2964 (1996). 
${ }^{23}$ P. Cloetens, W. Ludwig, J. Baruchel, D. V. Dyck, J. V. Landuyt, J. P. Guigay, and M. Schlenker, Appl. Phys. Lett. 75(19), 2912-2914 (1999).

${ }^{24}$ A. Bronnikov, Opt. Commun. 171, 239-244 (1999).

${ }^{25}$ M. A. Anastasio, D. Shi, Y. Huang, and G. Gbur, J. Opt. Soc. Am. A 22(12), 2651-2661 (2005).

${ }^{26}$ T. E. Gureyev, Y. I. Nesterets, K. M. Pavlov, and S. W. Wilkins, J. Opt. Soc. Am. A Opt. Image Sci. Vis 24(8), 2230-2241 (2007).

${ }^{27}$ T. E. Gureyev, A. Pogany, D. M. Paganin, and S. W. Wilkins, Opt. Commun. 231(1-6), 53-70 (2004).

${ }^{28} \mathrm{~F}$. Natterer, The Mathematics of Computerized Tomography (B. G. Teubner, Stuttgart, 1986).

${ }^{29}$ D. Paganin, S. C. Mayo, T. E. Gureyev, P. R. Miller, and S. W. Wilkins, J. Microsc. 206(Pt 1), 33-40 (2002).

${ }^{30}$ X. Wu, H. Liu, and A. Yan, Opt. Lett. 30(4), 379-381 (2005).

${ }^{31}$ T. E. Gureyev, D. M. Paganin, G. R. Myers, Y. I. Nesterets, and S. W. Wilkins, Appl. Phys. Lett. 89(3), 034102 (2006).

${ }^{32}$ L. Turner, B. Dhal, J. Hayes, A. Mancuso, K. Nugent, D. Paterson, R. Scholten, C. Tran, and A. Peele, Opt. Express 12(13), 2960-2965 (2004).

${ }^{33}$ T. E. Gureyev, A. W. Stevenson, D. M. Paganin, T. Weitkamp, A. Snigirev, I. Snigireva, and S. W. Wilkins, J. Synchrotron Radiat. 9, 148-153 (2002).

${ }^{34}$ M. A. Beltran, D. M. Paganin, K. Uesugi, and M. J. Kitchen, Opt. Express 18(7), 6423-6436 (2010).

${ }^{35}$ M. A. Beltran, D. M. Paganin, K. K. Siu, A. Fouras, S. B. Hooper, D. H. Reser, and M. J. Kitchen, Phys. Med. Biol. 56(23), 7353-7369 (2011).

${ }^{36} \mathrm{M}$. Langer, P. Cloetens, and F. Peyrin, IEEE Trans. Image Process. 19(9), 2428-2436 (2010).

${ }^{37}$ M. Langer, P. Cloetens, A. Pacureanu, and F. Peyrin, Opt. Lett. 37(11), 2151-2153 (2012).

${ }^{38}$ M. R. Teague, J. Opt. Soc. Am. 73(11), 1434-1441 (1983).

${ }^{39}$ T. Gureyev, D. Paganin, A. Stevenson, S. Mayo, and S. Wilkins, Phys. Rev. Lett. 93(6), 068103 (2004).

${ }^{40}$ T. E. Gureyev, Y. I. Nesterets, D. M. Paganin, A. Pogany, and S. W. Wilkins, Opt. Commun. 259(2), 569-580 (2006).
${ }^{41}$ T. Weitkamp, D. Haas, D. Wegrzynek, and A. Rack, J. Synchrotron Radiat. 18, 617-629 (2011).

${ }^{42}$ T. E. Gureyev, T. J. Davis, A. Pogany, S. C. Mayo, and S. W. Wilkins, Appl. Opt. 43(12), 2418-2430 (2004).

${ }^{43}$ L. A. Feldkamp, L. C. Davis, and J. W. Kress, J. Opt. Soc. Am. A 1(6), 612-619 (1984)

${ }^{44}$ G. Myers, S. Mayo, T. Gureyev, D. Paganin, and S. Wilkins, Phys. Rev. A 76(4), 045804 (2007).

${ }^{45}$ A. Groso, M. Stampanoni, R. Abela, P. Schneider, S. Linga, and R. Müller, Appl. Phys. Lett. 88(21), 214104 (2006).

${ }^{46}$ T. W. Baillie, T. E. Gureyev, J. A. Schmalz, and K. M. Pavlov, Opt. Express 20(15), 16913-16925 (2012).

${ }^{47}$ S. Brennan and P. L. Cowan, Rev. Sci. Instrum. 63(1), 850-853 (1992); A. W. Stevenson, Acta Cryst. A 49(1), 174-183 (1993); see http://www.tsimaging.net/Services/Simple/Default.aspx for an on-line calculator of the complex refractive index (accessed 4 September 2013).

${ }^{48} \mathrm{P}$. Cloetens, M. Pateyron-Salomé, J. Y. Buffière, G. Peix, J. Baruchel, F. Peyrin, and M. Schlenker, J. Appl. Phys. 81(9), 5878-5886 (1997).

${ }^{49}$ M. N. Boone, Y. D. Witte, M. Dierick, A. Almeida, and L. V. Hoorebeke, Microsc. Microanal. 18(2), 399-405 (2012)

${ }^{50}$ Y. I. Nesterets and T. E. Gureyev, "Noise propagation in X-ray phasecontrast imaging and computed tomography," J. Phys. D: Appl. Phys. (submitted).

${ }^{51}$ T. E. Gureyev, A. W. Stevenson, Y. I. Nesterets, and S. W. Wilkins, Opt. Commun. 240(1-3), 81-88 (2004).

${ }^{52}$ A. W. Stevenson, T. E. Gureyev, D. Paganin, S. W. Wilkins, T. Weitkamp, A. Snigirev, C. Rau, I. Snigireva, H. S. Youn, I. P. Dolbnya, W. Yun, B. Lai, R. F. Garrett, D. J. Cookson, K. Hyodo, and M. Ando, Nucl. Instrum. Methods Phys. Res. B 199, 427-435 (2003).

${ }^{53}$ T. E. Gureyev, S. C. Mayo, D. E. Myers, Y. Nesterets, D. M. Paganin, A. Pogany, A. W. Stevenson, and S. W. Wilkins, J. Appl. Phys. 105(10), 102005 (2009). 
Journal of

Synchrotron

Radiation

ISSN 1600-5775

Received 4 December 2013

Accepted 25 April 2014

\section{Quantitative evaluation of a single-distance phase-retrieval method applied on in-line phase-contrast images of a mouse lung}

\author{
Sara Mohammadi, ${ }^{\text {a,b* }}$ Emanuel Larsson, ${ }^{\mathrm{b}, \mathrm{c}, \mathrm{d}}$ Frauke Alves, ${ }^{\mathrm{e}}$ Simeone Dal Monego, ${ }^{\mathrm{f}}$ \\ Stefania Biffi, ${ }^{g}$ Chiara Garrovo, ${ }^{g}$ Andrea Lorenzon, ${ }^{f}$ Giuliana Trombab ${ }^{b}$ and \\ Christian Dullin ${ }^{\mathrm{h}}$ *
}

${ }^{\mathbf{a}}$ The Abdus Salam International Centre for Theoretical Physics, Trieste, Italy, ${ }^{\mathbf{b}}$ Synchrotron Light Source 'Elettra' Trieste, Strada Statale 14, km 163.5 in AREA Science Park, Basovizza 34149, Italy, 'Department of Physics, Chemistry and Biology, Linköping University, SE-581 83, Sweden, ${ }^{\mathbf{d} D e p a r t m e n t}$ of Architecture and Engineering, University of Trieste, Trieste, Italy, ${ }^{\mathbf{e}}$ Department of Hematology/Oncology, University Hospital Goettingen, Robert Koch Strasse 40, Goettingen, Lower Saxony 37075, Germany, ${ }^{\mathbf{f}}$ Cluster in Biomedicine s.c.r.l., AREA Science Park, Strada Statale 14, km 163.5, Basovizza, 34149 Trieste, Italy, '̊nstitute for Maternal and Child Health, IRCCS Burlo Garofolo, via dell'Istria 65/1, 34137 Trieste, Italy, and hepartment of Diagnostic and Interventional Radiology, University Hospital Goettingen, Robert Koch Strasse 40, Goettingen, Lower Saxony 37075, Germany. *E-mail: sara.mohammadi@elettra.trieste.it, christian.dullin@med.uni-goettingen.de

Propagation-based X-ray phase-contrast computed tomography (PBI) has already proven its potential in a great variety of soft-tissue-related applications including lung imaging. However, the strong edge enhancement, caused by the phase effects, often hampers image segmentation and therefore the quantitative analysis of data sets. Here, the benefits of applying single-distance phase retrieval prior to the three-dimensional reconstruction $(\mathrm{PhR})$ are discussed and quantified compared with three-dimensional reconstructions of conventional PBI data sets in terms of contrast-to-noise ratio (CNR) and preservation of image features. The PhR data sets show more than a tenfold higher CNR and only minor blurring of the edges when compared with PBI in a predominately absorption-based set-up. Accordingly, phase retrieval increases the sensitivity and provides more functionality in computed tomography imaging.

Keywords: computed tomography; phase-contrast imaging; phase retrieval; lung imaging.

\section{Introduction}

Within the aim of unravelling the patho-mechanism of lung disease and the testing of novel treatments, there is still a strong need for improvement of lung imaging techniques and their application in preclinical disease models. Owing to the very nature of the lung with its air-tissue interfaces, lung imaging remains challenging for most imaging modalities (Kauczor \& Kreitner, 1999). Propagation-based phasecontrast computed tomography (PBI) has already been proven valuable in applications focusing on low-absorbing tissue ('soft tissue') (Kitchen et al., 2005; Sera et al., 2005). The obtained edge effects facilitate the delineation of the airways, but on the other hand hamper or prohibit further quantitative analysis relying on threshold-based segmentation of the data sets. To circumvent this problem, edge-suppression techniques or low-pass filters can be used to remove these effects. However, this also diminishes the quality of the image features, especially for edges. Here, we propose and validate the application of a single-distance phase-retrieval method (Paganin et al., 2002) for in-line phase-contrast computed tomography (CT) imaging of a mouse lung in situ filled with air at a physiological pressure. Several other phase-retrieval techniques utilizing multiple sample-to-detector distances (Mayo et al., 2012; Kostenko et al., 2013; Cloetens et al., 1999) have been utilized before but are not practical for several reasons: e.g. they need an advanced imaging set-up and are very sensitive to variations in the incident beam, an aspect which needs to be considered at synchrotron light sources. Additionally, the movement and differences in the total amount of optical energy between projections acquired at different distances causes slight shifts and results in further alterations and artefacts. More importantly, multiple measurements increase the exposure time and dose delivered to the biological sample. Therefore, a single-distance phaseretrieval algorithm based on the transfer of intensity equation (Paganin et al., 2002; Gureyev et al., 2009; Teague, 1983), which only requires one $\mathrm{CT}$ data set obtained at a single sample-to- 
detector distance, has many advantages. This algorithm reconstructs the three-dimensional distribution of the complex refractive index, $\tilde{n}(r)=1-\delta(r)+i \beta(r)$, inside the sample by using X-ray projection images acquired at different view angles (rotational position of the sample). This class of algorithms can strictly speaking only be applied for 'homogeneous' objects, which are either pure absorption, pure phase objects or objects characterized by a constant ratio of the real to the imaginary parts of the refractive index, i.e. $\delta(r) / \beta(r)=\gamma=\mathrm{a}$ constant (Paganin et al., 2002). Although biological samples do not satisfy this assumption, it has been demonstrated by $\mathrm{Wu}$ et al. (2005) that these kinds of algorithms can still be applied for biological samples which are predominately composed of materials with a low atomic number $(Z<10)$, referred to as 'soft tissue' within this article. Here we use an in situ mouse lung sample and show that, even in the presence of material with $Z>10$, such as bone, the image quality can be dramatically increased by single-distance phase retrieval and exceeds that of PBI.

\section{Methods}

\subsection{Sample preparation}

The chest imaged in this study was taken from a mouse sacrificed using a xylazine-tiletamine-zolazepam overdose. In order to reproduce conditions which resemble the in vivo situation, the lung was inflated in situ with air, under a constant pressure of $30 \mathrm{~cm}$ water column $(2.94 \mathrm{kPa})$, through a series of smaller tubings, down to a polyethylene cannula (PE50) fixed inside the trachea with a cotton wire. To block the air inside the lung the trachea was tied up. Following this procedure the sample was kept at room temperature for $2 \mathrm{~h}$, in order to avoid any 'rigor mortis'-based alterations. In the final step the sample was embedded in $1 \%$ agarose gel, inside a $30 \mathrm{ml}$ falcon tube (Fisher Scientific, USA) serving as a sample holder, thus avoiding air leakage, alterations and movement during the time course of the X-ray examination. The agarose gel was left to set for another $30 \mathrm{~min}$ at $277 \mathrm{~K}$, which allowed for a complete gelatinization of the gel. Following this procedure the sample was placed inside the SYRMEP beamline experimental hutch $30 \mathrm{~min}$ before imaging, thus allowing for temperature adaptation in order to suppress any alterations of the lung during the scanning process.

\subsection{Data acquisition}

The chest area of the sample was scanned at the SYRMEP beamline of the Elettra synchrotron light source (Trieste, Italy). The sample was scanned at three sample-to-detector distances of $7 \mathrm{~cm}, 30 \mathrm{~cm}$ and $100 \mathrm{~cm}$, with the following parameters: X-ray energy $=22 \mathrm{keV}$, with a spatial resolution of $9 \mu \mathrm{m}$; field of view of $18 \mathrm{~mm} \times 12 \mathrm{~mm} ; 1800$ projections over a full rotation of $360^{\circ}$.

\subsection{Phase retrieval}

Owing to phase contrast, the obtained projection images display a mix of absorption-based contrast and edge effects whose magnitudes depend on the sample-to-detector distance. In order to enable threshold-based segmentation and to fully exploit the potential of phase-contrast CT, it is necessary to calculate an image which is predominated by the real part of the complex refractive index and without the edge effects. Therefore, to reconstruct the complex refractive index $\tilde{n}(r)$ in the sample, a single-distance phase-retrieval algorithm, based on the transfer of intensity equation (TIE), is applied to the acquired data sets (Paganin et al., 2002). Furthermore, only one scan per sample is needed, thus enabling an overall scanning time of about $1.5 \mathrm{~h}$ in a $360^{\circ}$ mode and therefore reduces artefacts based on alterations of biological samples over time. We used a TIE phase-retrieval algorithm implemented in the $X$-Tract software package developed at CSIRO (Paganin et al., 2002).

For this algorithm a priori knowledge of the ratio $(\gamma)$ between $\beta$ and $\delta$ of the refractive index is needed. Here we used $\gamma=1950$ for lung tissue. This value is based on the standardization of lung tissue by the International Commission on Radiological Protection (ICRP) which is described by hydrogen, carbon and oxygen in the following ratios: H 10, C 0.83, O 5 (Berger, 1992). This soft-tissue equivalent was used in the online calculator for the refractive index (Center of X-ray Optics, Lawrence Berkeley National Laboratory, http://henke.lbl.gov/optical_constants/getdb2. html) to obtain $\gamma$.

To evaluate the benefit of single-distance phase retrieval over conventional PBI, slices were also reconstructed without prior application of a phase-retrieval algorithm.

\subsection{Post-processing and quantification}

All scans were reconstructed after application of the TIE phase-retrieval algorithm $(\mathrm{PhR})$ and without phase retrieval (PBI). For quantitative analysis the PBI and PhR data sets were registered to the PBI $7 \mathrm{~cm}$ data set using a two-dimensional cross-correlation evaluation in order to identify the corresponding slice and a Fourier-Mellin algorithm to detect in-plane scaling, rotation and translation (Zitová \& Flusser, 2003). The two-dimensional cross correlation used as a measure for similarity between two images was strongly influenced by the edge effects in the PBI data sets and prevented the Fourier-Mellin algorithm from converging. Therefore, the PBI data sets were filtered only for the registration process by using a normal mean filter (size $3 \times 3 \times 3$ voxels) to suppress the edge effects. Profiles at air-tissue interfaces were calculated to assess the edge quality in the images. Standard deviations and mean values were measured in different volumes of interest $\left(0.4 \mathrm{~mm}^{2}\right)$ for fat, air, soft tissue and bone in all data sets. The contrast-to-noise ratio (CNR) between two adjoined tissues was calculated based on equation (1) (Muhogora et al., 2008).

In order to assess the quality of the edges in the images, the edge-enhancement index [EEI; equation (2)] (Donnelly et al., 2006) was calculated. However, the highest and lowest value ( $P$ and $L)$ on a profile plot used in the proposed equation (2) by Donnelly et al. is difficult to define within the sometimes 
sparsely sampled line profile, especially in the presence of the edge effects of the PBI images. Thus, we introduced a measure based on a non-linear fit of a sigmoid function [equation (3)] on these profiles. In order to avoid the registration process from influencing the noise level determination, the noise level was measured in the original untransformed data.

$$
\mathrm{CNR}=\frac{\overline{g_{1}}-\overline{g_{2}}}{\left[(1 / 2)\left(\sigma_{1}^{2}+\sigma_{2}^{2}\right)\right]^{1 / 2}},
$$

where $\overline{g_{1}}$ and $\overline{g_{2}}$ are the mean intensity values of a given homogenous area (size $0.4 \mathrm{~mm}^{2}$ ) in tissue and in air, respectively, and $\sigma_{1}$ and $\sigma_{2}$ are the corresponding standard deviations;

$$
\mathrm{EEI}=\frac{P-L}{\left(\sigma_{1}^{2}+\sigma_{2}^{2}\right)^{1 / 2}},
$$

where $P$ and $L$ are the highest and lowest values on a profile plot of an air-tissue interface (length $0.1 \mathrm{~mm}$ ), and $\sigma_{1}$ and $\sigma_{2}$ are the standard deviations of the profile regions depicting pure air and pure tissue, respectively;

$$
\operatorname{sig}(x)=\frac{k_{1}}{1+\exp \left[-k_{2}\left(x+k_{3}\right)\right]}+k_{4},
$$

where the different constants $k_{i}$ are used to adjust the sigmoid function to the present line profile. The steepness of the edge is depicted by the constant $k_{2}$.

\section{Results}

\subsection{Overall performance of the used phase-retrieval algorithm}

With increasing sample-to-detector distance the filtered back-projection (FBP) reconstruction of the PBI data sets reveals higher magnitudes of phase effects (Fig. 1a). Phase retrieval is meant to calculate the $\delta$-distribution (real part) of the complex refractive index within the sample and should therefore be independent of the sample-to-detector distance. The $\mathrm{PhR}$ results (Fig. $1 b$ ) in general show the expected behaviour apart from a slight increased blurring, thus indicating the successful application of the used algorithm.

\subsection{Quantitative comparison of the reconstructed phase- retrieved data sets with the $\mathrm{PBI}$ data sets}

In general, quantitative comparison is hampered by the fact that these two image types represent different features of the studied object: absorption plus edge enhancement in the PBI data sets, and phase-shift-dominated contrast without edge effects in the PhR data sets. Both can be advantageous in terms of the application of different image-processing protocols. Therefore, the following analysis is mainly meant to show the feasibility of using these data for threshold-based image segmentation or for visual inspection of the images. Thus, both contrast but also the quality of the edges of image features need to be addressed. The contrast is a measure of the effectiveness to discriminate between adjacent tissues and is positively dependent on the difference in the tissues' respective grey values and negatively influenced by the noise level. In order to quantify the image contrast and account for the presence of noise, the contrast-to-noise ratio [equation (1)] was measured.
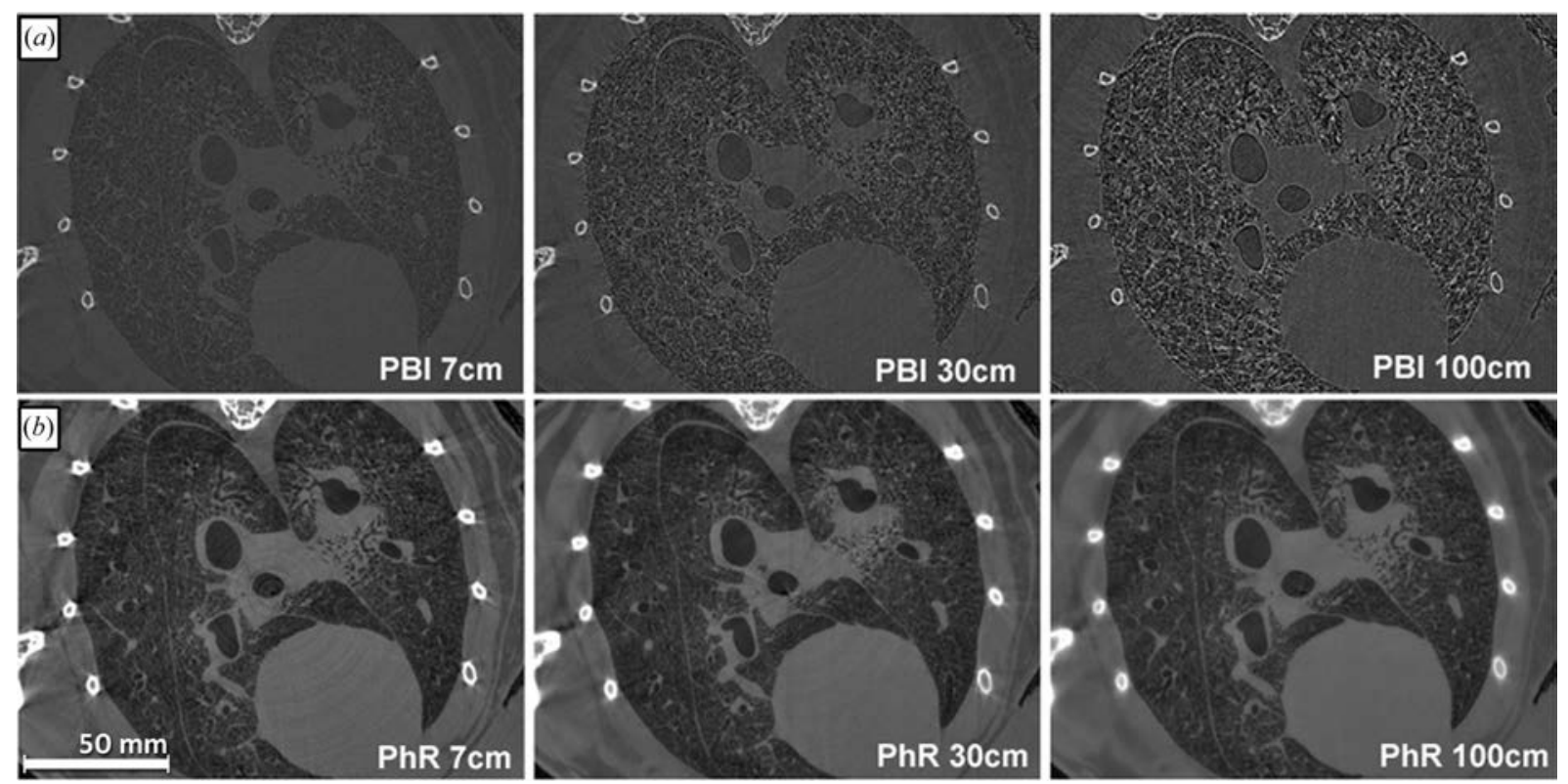

Figure 1

Comparison of the edge effects within the PBI data sets $(a)$ at 7,30 and $100 \mathrm{~cm}$ sample-to-detector distances. A clear increase of the edge effects can be seen with increasing distance. $(b)$ The respective results from the TIE phase-retrieval algorithm (PhR) with a $\delta$-to- $\beta$ ratio optimized for lung soft tissue, therefore bone structures appear blurred. Besides a slight increased blurring at $100 \mathrm{~cm}$ the images look alike and present a higher contrast than the PBI data sets. Note that only the central part of the data is shown for convenience. The full reconstruction represents the entire cross section of the sample. 
Table 1

Quantitative results of the image comparison.

\begin{tabular}{|c|c|c|c|c|c|c|}
\hline & \multicolumn{3}{|l|}{ PBI } & \multicolumn{3}{|l|}{$\mathrm{PhR}$} \\
\hline & $7 \mathrm{~cm}$ & $30 \mathrm{~cm}$ & $100 \mathrm{~cm}$ & $7 \mathrm{~cm}$ & $30 \mathrm{~cm}$ & $100 \mathrm{~cm}$ \\
\hline CNR air-soft-tissue & $1.55 \pm 0.23$ & $0.92 \pm 0.25$ & $0.73 \pm 0.25$ & $9.33 \pm 0.92$ & $17.54 \pm 1.77$ & $29.29 \pm 10.55$ \\
\hline EEI & $8.80 \pm 1.39$ & $12.45 \pm 0.80$ & $11.24 \pm 2.07$ & $25.41 \pm 2.29$ & $51.31 \pm 5.01$ & $63.03 \pm 2.02$ \\
\hline Steepness of fit & $57 \%$ & $100 \%$ & $15 \%$ & $8 \%$ & $7 \%$ & $5 \%$ \\
\hline
\end{tabular}

The same two-dimensional regions solely containing air or soft tissue (three different regions each, measured on six slices) with a size $0.4 \mathrm{~mm}^{2}$ were identified and their mean values and standard deviation were calculated in all data sets. These regions were selected away from tissue interfaces as to not be affected by the edges effects. The calculated CNR values show a high CNR between air and soft tissue within the lung of up to 29 for the phase-retrieved data set at $100 \mathrm{~cm}$ sample-to-detector distance (Table 1). Given the fact that the CNR increases with increasing sample-to-detector distance, this implies that imaging at higher distances may further enhance the results.

Additionally, five line profiles ( $0.2 \mathrm{~mm}$ length) at an airsoft-tissue interface were measured and the average of these line profiles was used to analyse the edge quality using equation (2) (EEI). As shown in Table 1, the EEI values for the PhR data sets are higher due to the strongly reduced noise of the profile. Therefore, EEI cannot reflect the true situation displayed in Fig. 2(a) compared with Fig. 2(b) which shows a much steeper and higher edge due to the edge effects in the $\mathrm{PBI}$ rather than in the $\mathrm{PhR}$ data sets.

Therefore, in order to quantify and compare the steepness of the edges and the influence of the edge effects we used a non-linear fitting regime for the measured profiles utilizing a sigmoid function [equation (3)] (Fig. 2, Table 1). Based on this equation the parameter $k_{2}$ reflects the steepness of the edge. In order to provide more intuitive values, the highest $k_{2}$ value (PBI $30 \mathrm{~cm}$ ) was set to $100 \%$ and all the other values were expressed as a ratio of this reference value (steepness-of-fit). In contrast to EEI, the steepness-of-fit parameter reflects the observed increase in blurring in the phase-retrieved data sets and shows a slight decrease from $8 \%$ for $7 \mathrm{~cm}$ to $5 \%$ for $100 \mathrm{~cm}$. This behaviour will hamper the use of very large sample-to-detector distances at least if a high spatial resolution in the range of the pixel size of the detection system is needed. Owing to the strong edge effects in the PBI data sets the edges appear steeper compared with the PhR data sets, ranging from $57 \%$ for $7 \mathrm{~cm}$ to $100 \%$ at $30 \mathrm{~cm}$. The breakdown in the edge steepness at $100 \mathrm{~cm}$ in the PBI data is caused by phase effects produced by the tissue texture, which carries more weight at greater sample-to-detector distances, and by the appearance of higher-order interference fringes, which cannot be properly sampled with the limited detector pixel size of $9 \mu \mathrm{m}$ (binning $2 \times 2$ used in this study). These effects create massive distortion of the measured edge profile which prevents the applicability of a fitting approach with a sigmoid function [equation (3)] and therefore diminishes the measured edge-steepness.

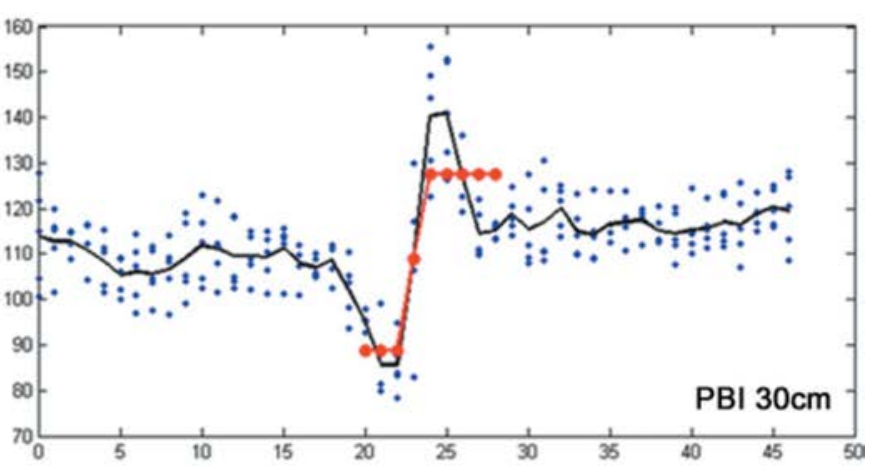

(a)

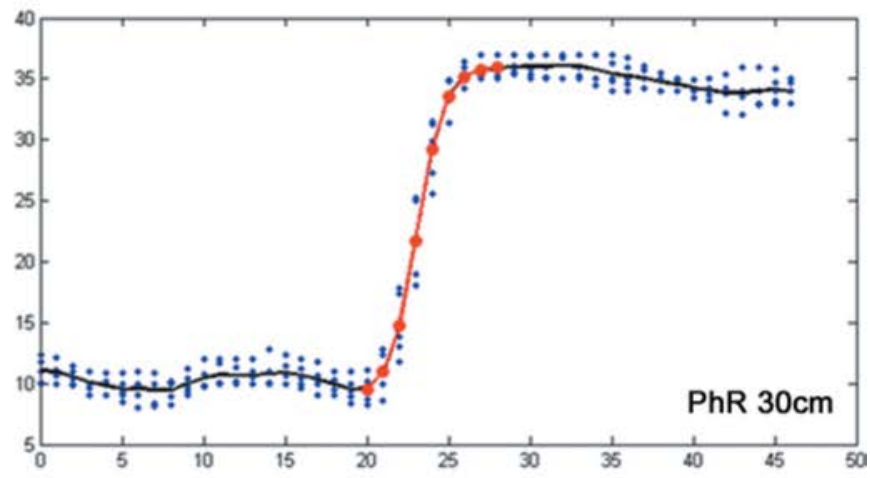

(b)

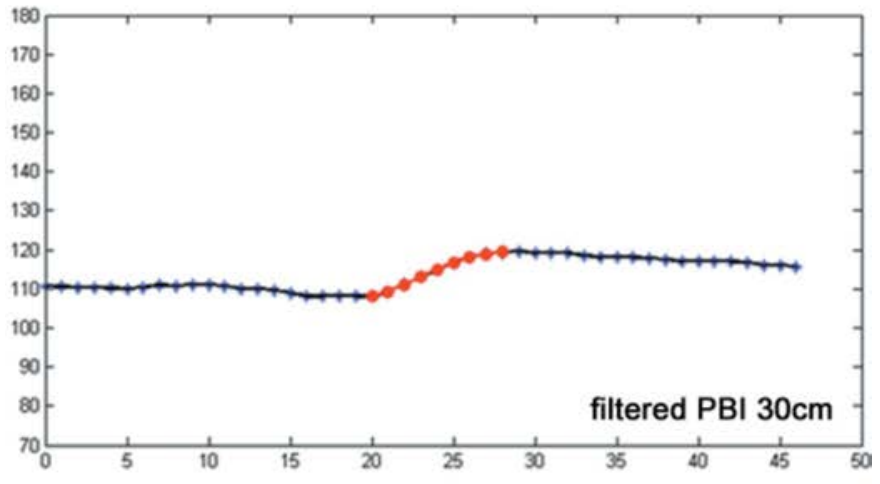

(c)

Figure 2

Comparison of the edge quality between PBI $(a), \mathrm{PhR}(b)$ and filtered PBI data sets $(c) .(a)-(c)$ show profile plots at the same location in PBI and $\mathrm{PhR}$ data sets obtained with a $30 \mathrm{~cm}$ sample-to-detector distance. In order to suppress the influence of noise, five individual profiles (blue dots) were measured and the average profile (black line) was used for evaluation. The red line resembles the fit of the sigmoid function [equation (3)]. A clearly steeper edge is apparent in $(a)$ due to the strong edge effects; $(b)$ shows a smoother edge but in combination with reduced noise; (c) presents the profile of the PBI data set after iterative use of a low-pass filter to reach the same edge steepness as in $(b)$. 


\subsection{Does phase retrieval do more than a low-pass filter applied to the raw data sets?}

As shown in Fig. 2(a), PBI at $30 \mathrm{~cm}$ is characterized by strong edge effects and therefore presents very steep edges compared with the phase-retrieval data set $\mathrm{PhR}$ of the same sample-to-detector distance (Fig. $2 b$ ). In order to prove that phase retrieval cannot be substituted by a simple low-pass filter to remove the edge effects, the PBI data set was gradually filtered using a Gaussian filter (kernel with 3 pixels) until the profile presented the same steepness as the PhR data set. Even in this ideal situation where the CNR is increased due to the suppressed noise, it only reaches about 5 as against 17.5 obtained with the PhR data set. This indicates that phase retrieval cannot be substituted by low-pass filtering.

\subsection{Single-distance phase-retrieval applied to in-line phase-contrast synchrotron-radiation-based CT data sets of an in situ mouse lung opens up for structural analysis of lung tissue}

Utilizing the TIE phase-retrieval algorithm we have reached a more than ten times higher CNR value in the images of an in situ mouse lung. Fig. 3 exemplifies the difference in the appearance of PBI and PhR data sets by showing the same slice at $30 \mathrm{~cm}$ cut in the middle. In Fig. 3(a), PBI depicts the clear delineation of the air to soft-tissue interface and the presence of strong edge effects. The blue line in the profile plot at position $\mathrm{P}$ shows the large variation of the grey values and the strong edge effect at the interface. In addition, the overall histogram of this slice allows no contrast-based separation of tissues, presenting only one Gaussian-shaped distribution [Fig. 3(b), blue histogram]. In contrast, the PhR data set in Fig. 3(b) shows no signs of edge effects. The profile plot (red) depicts a common stair-shaped function with low variation within the air and the soft-tissue plateau phase. The histogram clearly shows at least two components for air and soft tissue which enables threshold-based segmentation and therefore quantitative image analysis.

\section{Discussion}

Here we present the benefits of utilizing in-line phase-contrast $\mathrm{CT}$ for lung imaging in combination with single-distance phase retrieval as demonstrated on an in situ mouse lung sample. The application of in-line phase-contrast CT on lungs exploits the presence of the air-tissue interfaces and provides significantly better delineation of the airways than several other applications (Siu et al., 2008; Sera et al., 2005; Kitchen et al., 2005). On the other hand, the strong edge effects in the data sets hamper the segmentation of different components (air, soft tissue) and therefore prevent a quantitative analysis.

By utilizing single-distance phase retrieval, as demonstrated in this study, data sets can be generated which predominately show the distribution of the real part of the complex refractive index within the samples and do not display any edge effects. We verified the reliability of this approach by analysing the same lung sample at different sample-to-detector distances

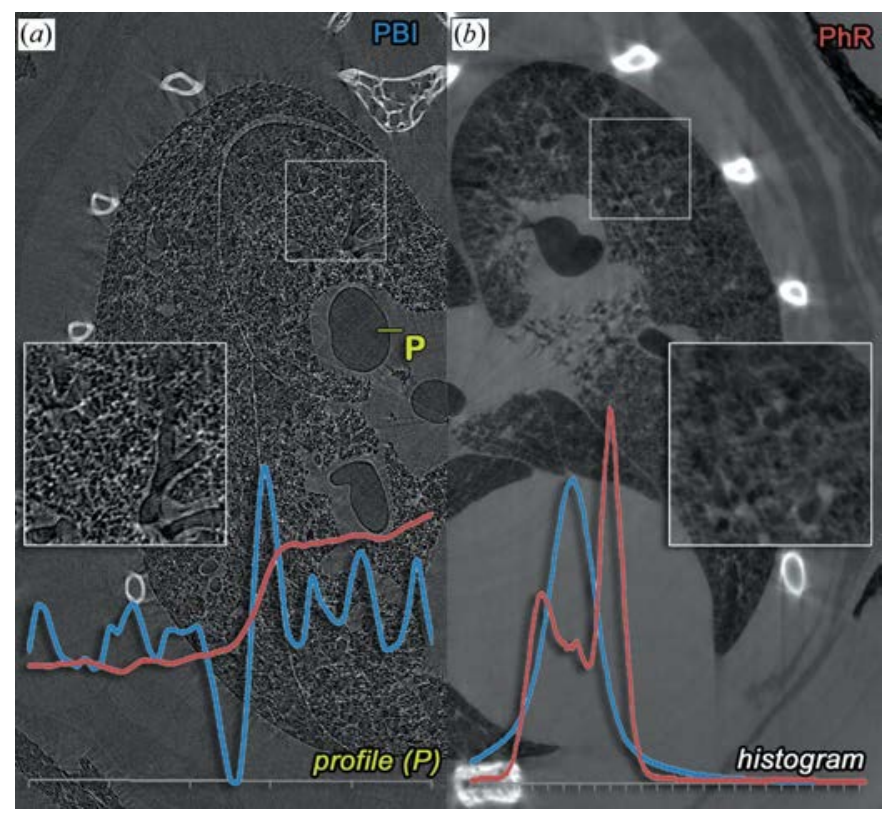

Figure 3

A direct comparison of the image features for PBI $(a)$ and the TIE phaseretrieved data set $(b)$ is shown, both obtained for the same sample at an object-to-detector distance of $30 \mathrm{~cm}$ (this figure depicts only the central part of the reconstruction for convenience). As indicated in the detailed views, PBI presents strong edge effects when compared with PhR. The overall grey value histograms (lower right corner) for $\mathrm{PBI}$ (blue) and $\mathrm{PhR}$ (red) show that, due to the phase effects overlaying the measured absorption, only one Gaussian-shaped peak can be seen. Therefore, no intensity-based segmentation between air and tissue can be performed. In contrast, after phase retrieval the histogram is clearly composed of two density intervals. The line profile at the position P in PBI (blue) and PhR (red) in the lower left-hand corner shows large variations and a strong edge effect for PBI, whereas in PhR the profile resembles the expected jump-function for a simple air-tissue interface. These drastic edge effects also cause negative values lower than the value for air, within the bronchi (dark contours). Therefore, the airways may appear filled, which is not the case. Note that the $\delta$-to- $\beta$ ratio for PhR was optimized for soft tissue and did not match the ratio of bone, which is why ribs and spine appear more blurred than in PBI.

and obtained the same results with every distance. Remarkably, the CNR of the generated data sets are more than ten times higher than with the classical absorption-based mode (short sample-to-detector distance). It has to be stated that the ten-fold gain in CNR is related to many different factors, including the overall set-up of the experiment, the characteristics of the chosen sample, the sample-to-detector distances, the resolution of the used detector system, characteristics of the incident X-ray beam, the used implementation of the reconstruction and the phase-retrieval software. Therefore, the calculated factor of ten does not represent a general rule when comparing phase-retrieved images with PBI images and may vary in other set-ups. Beltran et al. for instance reported a 9-200-fold increase in CNR (Beltran et al., 2011).

In addition, CNR is an image parameter, which can be easily increased by de-noising. This usually suppresses high spatial frequencies and therefore diminishes the quality of edges. Therefore, it should not be used for quantification of image quality without a measure of the preservation of image sharpness. We repeatedly applied mean filtering on the PBI 
images to reach the same CNR as measured by $\mathrm{PhR}$, but observed a dramatically lower sharpness of the edges than in $\mathrm{PhR}$. This demonstrates that single-distance phase retrieval cannot be substituted by filtering of PBI images.

Interestingly, in the normal PBI data sets we also observed a decrease in CNR with increasing sample-to-detector distance. This is in contrast to our previous findings from the analysis of a phantom filled with different substances and imaged with two sample-to-detector distances (Gureyev et al., 2013). We believe the loss in CNR is caused by the intrinsic small density variation within biological tissue, such as the lung. Even in areas solely composed of one tissue type, these variations cause additional phase effects which increase the image 'noise' and therefore diminish the CNR. This notion is supported by Donnelly et al. (2003), who quantitatively analysed the dependency of the observed phase effects of certain systemic parameters and found a strong impact of tissue texture and scattering on the detection of the phase effect fringes in biological samples. Our findings support these studies and underline the importance of evaluating novel imaging approaches in biological specimens.

Our data show that, even if biological samples do not fulfil the preconditions of a 'homogeneous' object (Gureyev et al., 2009) for single-distance phase-retrieval algorithms and the generated data sets therefore predominately reflect only the real part of the complex refractive index, the achieved image quality outperforms that of absorption-based CT and PBI (phase-contrast CT without phase retrieval). In addition, the same short imaging time can be maintained with this singledistance phase-retrieval approach, something that would be impossible with other algorithms requiring multiple sampleto-detector distances. However, as previously reported (Beltran et al., 2011), the application of this class of phaseretrieval algorithms requires a priori knowledge of the $\delta$-to- $\beta$ ratio of the refractive index of the analysed sample. In our study we accordingly chose the appropriate ratio for lung tissue $(\delta$-to- $\beta$ ratio $=1950)$. Therefore, the bone details, like the spine and rib cage, appear blurred in the reconstructions due to the fact that they are characterized by a $\delta$-to- $\beta$ ratio of about 250 (Center of X-ray Optics, Lawrence Berkeley National Laboratory, http://henke.lbl.gov/optical_constants/ getdb2.html), based on the composition of bone of $\mathrm{H} 0.06$, $\mathrm{C} 0.28, \mathrm{~N} \mathrm{0.3,} \mathrm{O} \mathrm{4.1,} \mathrm{P} \mathrm{7,} \mathrm{Ca} 15$ as found in the database of the National Institute of Standards and Technology (NIST). This underlines the fact that single-distance phase-retrieval algorithms cannot be used to calculate the $\delta$-value distribution of the refractive index in samples with a strong variance of $\delta$-to- $\beta$ ratios.

Another interesting result is that the analysed CNR in the phase-retrieved data rises with increasing sample-to-detector distances. This suggests that setting up imaging beamlines with greater sample-to-detector distances may improve the quality of such a lung imaging approach even further. The measured gain in CNR directly translates into an increased sensitivity, which will allow for precise three-dimensional analysis of morphological alterations within, for instance, mouse lung disease models. We therefore believe that the method presented here can be beneficial in a wide variety of similar preclinical studies.

SM acknowledges financial support from The Abdus Salam International Centre for Theoretical Physics (ICTP, Trieste), through the TRIL programme. All authors especially thank Dr T. Gureyev of CSIRO, Australia, for all the fruitful discussions and for providing access to the $X$-Tract software package used for phase retrieval in this study. The experiment was conducted within the European project 'Public Private Partnership for Asthma Imaging and Genomics' (P3AGI, http://www.p3agi.eu) funded by the European Commission through an FP7 IAPP Marie Curie Action (GA 230739). Besides the co-authors, every member of the SYRMEP team contributed to the success of the experiment, in particular Diego Dreossi and Nicola Sodini. Animal handling and preparation was performed at CBM in Trieste, Italy. The authors also thank Dr Andrea Markus (UMG, Göttingen, Germany) for proof-reading and spell-checking.

\section{References}

Beltran, M. A., Paganin, D. M., Siu, K. K. W., Fouras, A., Hooper, S. B., Reser, D. H. \& Kitchen, M. J. (2011). Phys. Med. Biol. 56, 7353-7369.

Berger, M. J. (1992). ESTAR, PSTAR, and ASTAR: Computer Programs for Calculating Stopping-Power and Range Tables for Electrons, Protons, and Helium Ions.NIST, Gaithersburg, MD, USA.

Cloetens, P., Ludwig, W., Baruchel, J., Van Dyck, D., Van Landuyt, J., Guigay, J. P. \& Schlenker, M. (1999). Appl. Phys. Lett. 75, 2912 2914.

Donnelly, E. F., Lewis, K. G., Wolske, K. M., Pickens, D. R. \& Price, R. R. (2006). Phys. Med. Biol. 51, 21-30.

Donnelly, E. F., Price, R. R. \& Pickens, D. R. (2003). Med. Phys. 30, 2888.

Gureyev, T. E., Mayo, S. C., Myers, D. E., Nesterets, Ya., Paganin, D. M., Pogany, A., Stevenson, A. W. \& Wilkins, S. W. (2009). J. Appl. Phys. 105, 102005.

Gureyev, T., Mohammadi, S., Nesterets, Y., Dullin, C. \& Tromba, G. (2013). J. Appl. Phys. 114, 144906.

Kauczor, H.-U. \& Kreitner, K.-F. (1999). Eur. Radiol. 9, 1755-1764.

Kitchen, M. J., Lewis, R. A., Yagi, N., Uesugi, K., Paganin, D., Hooper, S. B., Adams, G., Jureczek, S., Singh, J., Christensen, C. R., Hufton, A. P., Hall, C. J., Cheung, K. C. \& Pavlov, K. M. (2005). Br. J. Radiol. 78, 1018-1027.

Kostenko, A., Batenburg, K. J., Suhonen, H., Offerman, S. E. \& van Vliet, L. J. (2013). Opt. Express, 21, 710-723.

Mayo, S. C., Stevenson, A. W. \& Wilkins, S. W. (2012). Materials, 5, 937-965.

Muhogora, W. E., Devetti, A., Padovani, R., Msaki, P. \& Bonutti, F. (2008). Radiat. Protect. Dosim. 129, 231-236.

Paganin, D., Mayo, S. C., Gureyev, T. E., Miller, P. R. \& Wilkins, S. W. (2002). J. Microsc. 206, 33-40.

Teague, M. R. (1983). J. Opt. Soc. Am. 73, 1434-1441.

Sera, T., Uesugi, K. \& Yagi, N. (2005). Med. Phys. 32, 2787.

Siu, K. K. W., Morgan, K. S., Paganin, D. M., Boucher, R., Uesugi, K., Yagi, N. Parsons, D. W. (2008). Eur. J. Radiol. 68, S22-S26.

Wu, X., Hong, Liu, X. \& Yan, A. (2005). Opt. Lett. 30, 379-381.

Zitová, B. \& Flusser, J. (2003). Image Vis. Comput. 21, 977-1000. 


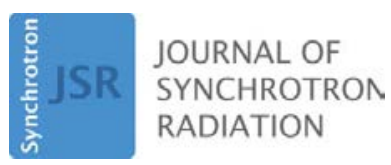

ISSN 1600-5775

Received 21 September 2014

Accepted 26 March 2015

Edited by A. Momose, Tohoku University, Japan

Keywords: phase-contrast CT; single-distance phase retrieval; lung imaging; asthma mouse models.

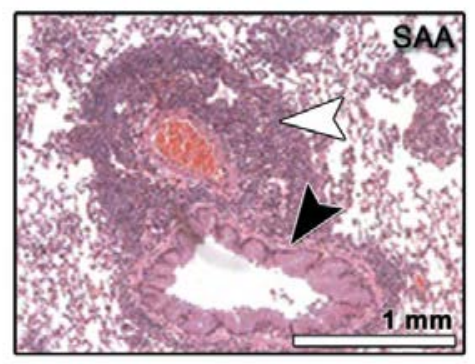

OPEN $\odot$ ACCESS

\section{Phase-contrast computed tomography for quantification of structural changes in lungs of asthma mouse models of different severity}

\author{
Christian Dullin, ${ }^{\text {a* Emanuel Larsson, },{ }^{\mathrm{b}, \mathrm{c}, \mathrm{d}} \text { Giuliana Tromba, }}{ }^{\mathrm{b}}$ Andrea M. Markus ${ }^{\mathrm{e}}$ \\ and Frauke Alves ${ }^{\mathrm{a}, \mathrm{e}, \mathrm{f}}$
}

\begin{abstract}
anstitute of Diagnostic and Interventional Radiology, University Medical Center Goettingen, Robert Koch Strasse 40, Goettingen, Lower Saxony 37075, Germany, 'b Elettra-Sincrotrone Trieste, Strada Statale 14, km 163,5 in AREA Science Park, Basovizza (Trieste) 34149, Italy, 'Department of Architecture and Engineering, University of Trieste, Trieste, Italy, ${ }^{\mathbf{d} D e p a r t m e n t}$ of Physics, Chemistry and Biology, Linkoeping University, SE-581 83 Linkoeping, Sweden, ${ }^{\mathbf{e} D e p a r t m e n t}$ of Haematology and Medical Oncology, University Medical Center Goettingen, Robert Koch Strasse 40, Goettingen, Lower Saxony 37075, Germany, and 'Department of Molecular Biology of Neuronal Signals, Max Planck Institut for Experimental Medicine, Hermann-Rein-Strasse 3, Goettingen, Lower Saxony 37075, Germany.

*Correspondence e-mail: christian.dullin@med.uni-goettingen.de
\end{abstract}

Lung imaging in mouse disease models is crucial for the assessment of the severity of airway disease but remains challenging due to the small size and the high porosity of the organ. Synchrotron inline free-propagation phase-contrast computed tomography (CT) with its intrinsic high soft-tissue contrast provides the necessary sensitivity and spatial resolution to analyse the mouse lung structure in great detail. Here, this technique has been applied in combination with single-distance phase retrieval to quantify alterations of the lung structure in experimental asthma mouse models of different severity. In order to mimic an in vivo situation as close as possible, the lungs were inflated with air at a constant physiological pressure. Entire mice were embedded in agarose gel and imaged using inline free-propagation phase-contrast CT at the SYRMEP beamline (Synchrotron Light Source, 'Elettra', Trieste, Italy). The quantification of the obtained phase-contrast CT data sets revealed an increasing lung soft-tissue content in mice correlating with the degree of the severity of experimental allergic airways disease. In this way, it was possible to successfully discriminate between healthy controls and mice with either mild or severe allergic airway disease. It is believed that this approach may have the potential to evaluate the efficacy of novel therapeutic strategies that target airway remodelling processes in asthma.

\section{Introduction}

Mouse lung disease models are widely used in preclinical asthma research (Bates et al., 2009). Despite certain limitations (Epstein, 2004), they are the method of choice to gain insight into the pathomechanism of this complex multifactorial disorder and to evaluate novel therapeutic concepts (Markus et al., 2014). However, the smallness of the lung, its high porosity and the minor alterations in the lung structure caused by asthma render classical imaging strategies like medical ultrasound, MRI and computed tomography (CT) extremely challenging. Synchrotron-radiation-based CT has been proven very effective in lung imaging (Lewis, 1997) and can provide spatial resolutions down to the sub-micrometre level. Studies conducted at the European Synchrotron Facility by Bayat et al. (2008) showed that the $K$-edge absorption technique using xenon as contrast agent is very effective for in vivo imaging of lung functionality. However, the used pixel 
size of $350 \mu \mathrm{m} \times 350 \mu \mathrm{m}$ did not allow an accurate evaluation of alterations in pulmonary morphology. Moreover, phasesensitive techniques such as free-propagation inline phasecontrast CT and grating interferometry imaging with their improved soft-tissue contrast (Beltran et al., 2011) have already been successfully applied for lung imaging by various groups such as Yagi, Kitchen, Hooper and Lewis (Kitchen et al., 2004; Hooper et al., 2007; Lewis et al., 2005; Yagi et al., 1999) to name but a few. Here, we used synchrotron radiation inline phase-contrast CT (XPCT) in combination with singledistance phase-retrieval algorithms for anatomical imaging of mouse lungs, as it provides a detailed high-contrast depiction of biological soft tissue while requiring only one tomographic acquisition. This approach has already been proven beneficial in lung imaging by analysing the phase shift of the X-ray incident beam within the sample (Parsons et al., 2008; Kitchen et al., 2005; Yong et al., 2009). Moreover, in a pilot study we demonstrated that with XPCT the contrast-to-noise ratio (CNR) in lung imaging can be increased by at least a factor of ten when compared with classical absorption-based CT (Mohammadi et al., 2014). In order to test the capability of XPCT to distinguish between minor and major lung alterations we chose two experimental allergic airway disease mouse models of different severity, one that resembles mild acute allergic asthma (Dullin et al., 2015) expressing only minor morphological changes and one that mimics severe asthma with dominant alterations of the lung structure (Nabe et al., 2005). Both models were previously reported by us and others (Markus et al., 2014; Dullin et al., 2015; Biffi et al., 2013; Bosnjak et al., 2014) and exhibit reproducible numbers of eosinophils in bronchoalveolar lavage as well as a consistent amount of cell infiltration in histology.

We show that XPCT can discriminate between the two airway disease models and that this technique provides the necessary sensitivity for quantitative analysis of structural differences in the lungs by comparing parameters like softtissue content and changes in the lung tissue composition, parameters that correlate with typical hallmarks of asthma and thereby with the severity of the disease.

\section{Methods}

Female BALB/c mice (4-6 weeks old) were purchased from Harlan Laboratories and maintained with ad libitum food and water. Two experimental allergic airway disease models of different severity were generated to mimic 'mild' acute allergic asthma (MAA) (Markus et al., 2014; Biffi et al., 2013; Dullin et al., 2015) and 'severe' acute allergic asthma (SAA) (Nabe et al., 2005). For induction of MAA, mice were sensitized twice intraperitoneally (i.p.) with $10 \mu \mathrm{g}$ ovalbumin (OVA) in $200 \mu \mathrm{l}$ phosphate-buffered saline (PBS) on days 0 and 21. For SAA, mice were sensitized on days 0 and 14 i.p. with a mixture of $50 \mu \mathrm{g}$ OVA and $0.5 \%$ of aluminium hydroxide adjuvant (Invivogen, San Diego, USA) in a volume of $200 \mu \mathrm{l}$ PBS, as well as intranasally (i.n.) with $50 \mu \mathrm{g}$ OVA in $25 \mu \mathrm{l}$ PBS. In order to provoke an acute allergic reaction, mice were treated i.n. either with a solution of $100 \mu \mathrm{g}$ OVA/50 $\mu \mathrm{l} \mathrm{PBS} /$ mouse
(MAA, at days 28 and 29) or with a solution of $250 \mu \mathrm{g}$ OVA/ $50 \mu \mathrm{l} \mathrm{PBS} /$ mouse (SAA, at days 28, 29, 30, and 33). The control group $(\mathrm{CN})$ was composed of mice which received PBS only, following the schedule of the MAA model. Each group contained four mice. All animal in vivo procedures were performed at the University Medical Center Goettingen, Germany, in compliance with the guidelines of the European (86/609/EEC) and the German ethical laws and were approved by the administration of Lower Saxony, Germany.

Mice were sacrificed two days after the last challenging step, i.e. on day 31 (MAA, CN) and on day 35 (SAA). In order to mimic the in vivo properties of the lung as close as possible, samples were prepared for the phase-contrast CT analysis as described before (Dullin et al., 2015) by performing a tracheotomy on the sacrificed mice, followed by inflation of the lungs with air at a constant pressure of $30 \mathrm{~cm}$ water column. Finally, tracheas were tied up and the whole mice were embedded in $1 \%$ agarose gel in $30 \mathrm{ml}$ falcon tubes.

The samples were imaged at the SYRMEP beamline (Synchrotron Light Source 'Elettra', Trieste, Italy) with the following parameters: X-ray energy $E=22 \mathrm{keV}, 1800$ projections in a $360^{\circ}$ acquisition mode using an exposure time per projection of $2.4 \mathrm{~s}$. For detection, we utilized a water-cooled CCD camera (Photonic Science, model VHR) with a $4008 \times$ 2672 full frame in $2 \times 2$ binning mode (resulting in a pixel size of $9 \mu \mathrm{m} \times 9 \mu \mathrm{m}$ ), coupled to a gadolinium oxysulfide scintillator placed on a fibre optic taper. The agarose embedded mice were mounted in an upright position and their central lung part $(\sim 4 \mathrm{~mm}$ in height $)$ was imaged. A sample-todetector distance of $30 \mathrm{~cm}$ was chosen to allow for inline phase-contrast measurements. In order to decouple the phase shift from the absorption effect, a single-distance phaseretrieval algorithm [TIE_Hom (Gureyev et al., 2009; Paganin et al., 2002), $X$-tract software package, CIRS, Australia] was applied to the projection images (Mohammadi et al., 2014) before reconstruction using a classical filtered back-projection algorithm (FBP). This resulted in three-dimensional (3D) data sets, predominately representing the real part of the complex refractive index, demonstrating a high CNR as well as a good edge quality (Mohammadi et al., 2014).

CT imaging results were correlated to histology. For this purpose, lung samples were obtained from a further set of OVA-induced asthmatic and control mice following the same asthma induction protocols. Excised lungs were fixed in $10 \%$ buffered formalin and embedded in paraffin. $3 \mu \mathrm{m}$-thick paraffin lung sections containing main stem bronchi were obtained and hematoxylin-eosin (H\&E) stained for $2 \mathrm{~min}$. Finally, these stained sections were dehydrated using an ascending alcohol series and xylol followed by mounting with DePex (Serva, Heidelberg, Germany). An Axioskop 2 (Carl Zeiss Microscopy $\mathrm{GmbH}$, Jena, Germany) microscope in combination with a Leica DC 100 camera (Leica, Switzerland) was used for visualization of the stained sections.

For quantification of differences in the water content of the lung tissue from asthmatic and healthy mice an additional cohort of age-matched mice was used (MAA, $N=6$; SAA, $N=5 ; \mathrm{CN}, N=6$ ), that were immunized and challenged 
following the same protocol as the mice analysed by CT and histology. Lungs from these mice were excised at the same time point on day 31 (MAA, CN) and on day 35 (SAA). One half of the lung was fixed in $10 \%$ buffered formalin for later histological verification of the presence or absence of asthma. The other half was weighed directly after explantation (wet) and after a $24 \mathrm{~h}$ drying process (dry) using a vacuum concentrator (SpeedVac ${ }^{\mathrm{TM}}$, ThermoSientific).

\section{Results and discussion}

By applying inline phase-contrast CT in combination with single-distance phase retrieval we found that the differences in severity of the disease in these two experimental allergic airway models in comparison with healthy controls are reflected in an increase in the soft-tissue content of the lung. Fig. 1 shows representative images of the soft-tissue-air interface of lungs from one mouse of each group (CN, MAA and SAA) rendered in $3 \mathrm{D}$ using the same parameters. The observed increase in the soft-tissue content correlates with the severity of the analysed models. In order to quantify these alterations in the lung structure, eight non-overlapping volumes-of-interests (VOIs) of $2 \mathrm{~mm} \times 2 \mathrm{~mm} \times 2 \mathrm{~mm}$ were placed uniformly in the peripheral region of the lung in order to depict comparable alveolar structures and ensure reproducibility of the measurement in different samples. The grey value histogram of such a VOI shows two dominant peaks representing air and soft tissue. The mean value between these two peaks was chosen as a threshold to discriminate air from soft tissue and the threshold was kept constant for all VOIs and samples. The volume ratio (Vol.Ratio) of the soft-tissue component was calculated by dividing the total volume of all voxels within the soft-tissue threshold range within the VOI by the VOI volume. The values of the eight VOIs per sample were averaged and the results are shown in Fig. 2(a). The three groups (CN, MAA and SAA) are clearly distinguishable by a statistically significant (one-way ANOVA test, $p$-value $<0.01$; Fig. $2 a$ ) increase in the soft-tissue Vol.Ratio in correlation with the severity of the analysed models $(0.2$ in $\mathrm{CN}, 0.33$ in MAA and 0.55 in SAA).

The success of our imaging approach to discriminate between diseased and healthy mice is vastly dependent on our preparation scheme (Dullin et al., 2015) which is designed to minimize alterations in the samples during the scanning procedure. Inflating the lung with air at a constant pressure results in an increased total lung volume (soft-tissue and air, our own previous observation) and is most likely caused by reduced elasticity of the lung tissue in asthmatic mice, already described by Gelb et al. (2002). Therefore, the increased softtissue volume ratio, which was found to be significantly higher in the diseased mice, still underestimates the effect in vivo. These ex vivo findings must not be confused with the so-called 'air-trapping' effect, a symptom often seen in asthmatic patients and used as a clinical parameter in CT-based asthma diagnosis. 'Air trapping' actually increases the air content in certain lung areas at the expiration phase due to a reduced ability to expel air (Busacker et al., 2009).

Another important aspect that has to be considered is that asthma is accompanied by an increased mucus production. The capabilities of our imaging strategy are limited in terms of discriminating mucus from soft tissue. Therefore, the analysed total amount in the tissue content of the asthmatic lungs may be somewhat smaller if mucus is present. However, the measurement of the air content of the asthmatic lungs is unaffected by this limitation and suggests that the analysed Vol.Ratio of non-air components within the lung represents a good parameter for the characterization of the severity of the allergic reaction in each lung sample.

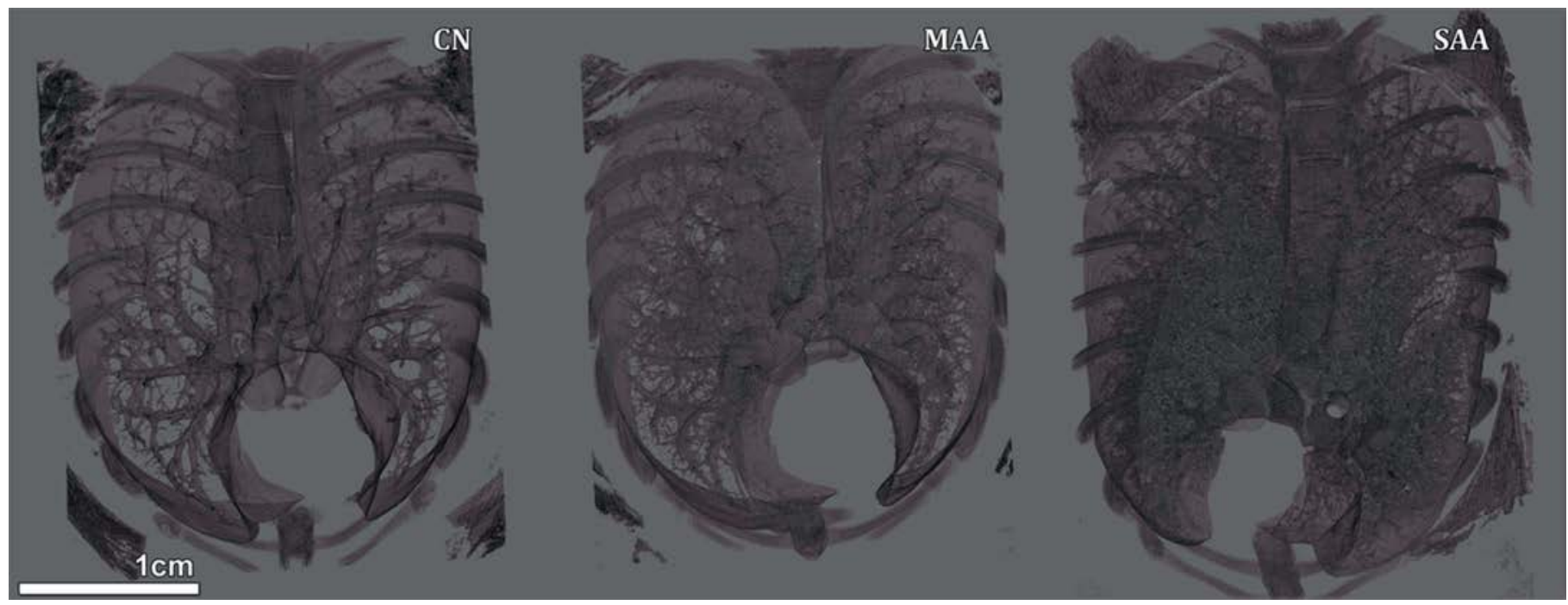

Figure 1

Volume rendering representations of the obtained XPCT results for lungs from a healthy control mouse (CN), a mouse from the mild (MAA) and a mouse from the severe (SAA) experimental allergic airway model. Increased soft-tissue content is clearly visible within lungs in correlation with increasing severity of asthma. 

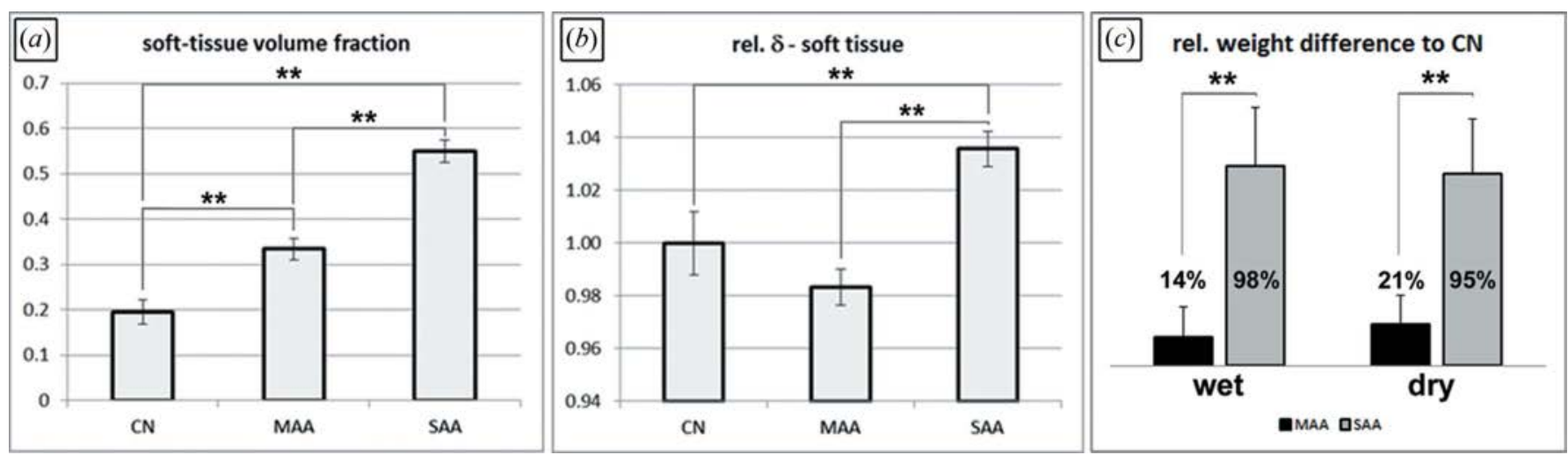

Figure 2

(a) The measured soft-tissue volume fractions obtained in eight VOIs per mouse are shown. Note that the same trend of increasing soft-tissue content seen in Fig. 1 can be found here. (b) The relative mean $\delta$-values of the lung soft tissue (normalized to the control group CN) are demonstrated. An increase of the relative $\delta$-value is found in lungs of mice with severe asthma (SAA). In contrast, lungs of mice with mild asthma (MAA) demonstrate slightly reduced relative $\delta$-values. (c) Relative difference of the lung weight of MAA $(N=6)$ and SAA $(N=5)$ compared with CN $(N=6)($ additional cohort of mice) directly after explantation (wet) and after being dried for $24 \mathrm{~h}$ (dry). Lungs of SAA mice weighed almost twice as much as lungs of CN with a small reduction in dry weight, indicating that the difference is equally related to more cells and higher water content. The relative weight difference of MAA increases from $14 \%$ to $21 \%$ from the wet to dry state, indicating that this effect is much smaller than in SAA and is slightly more related to an increase in the number of cells within the lung than to a higher water content. The error bars represent the standard deviation of the respective values within the different groups. ** indicates a $p$-value of a one-way ANOVA test of less than 0.01 and therefore a significant difference.

In order to validate the observed increase in soft-tissue content in asthmatic lungs, sections of lungs explanted four days after the last challenge were histologically analyzed. Fig. 3 shows representative results for one sample of each group. In accordance with other reports, we found an increased thickness of the bronchial walls, which is associated with the severity of the allergic inflammatory reaction in mice in these asthmatic mouse models (Dullin et al., 2015). In addition, areas with a high cell density as shown in Fig. 3 can only be found in SAA samples, confirming the strongly increased soft-tissue Vol.Ratio depicted by phase-contrast CT in SAA.

The application of a phase-retrieval algorithm results in $3 \mathrm{D}$ data sets which predominately express the real part ( $\delta$-value) of the refractive index within the samples (Gureyev et al., 2013). Since the $\delta$-value is characteristic of a certain element, the mean grey value of the segmented soft tissue contains information about the chemical tissue composition. Note that, whereas the imaginary part of the refractive index can quite reliably be estimated by the used phase-retrieval algorithm (TIE-Hom), the $\delta$-value depends on the chosen $\delta$-to- $\beta$ ratio as indicated by Gureyev et al. (2013). Therefore, there is no direct relation of the calculated grey values to the $\delta$-value. However, since the same $\delta$-to- $\beta$ ratio was used for all samples, a similar grey level can be expected in all groups if the lung soft tissue has the same composition. Here, in order to facilitate the comparison between the three groups, the individual average grey values for the eight VOIs analysed per sample were normalized to the mean value of the $\mathrm{CN}$ group as shown in Fig. 2(b).

A slightly $(2 \%)$ reduced mean $\delta$-value of lung soft tissue was found in MAA and an (4\%) increased mean $\delta$-value in SAA mice [one-way ANOVA test, $p$-value $<0.01$, Fig. $2(b)$ ], pointing to differences in the airway remodelling mechanism
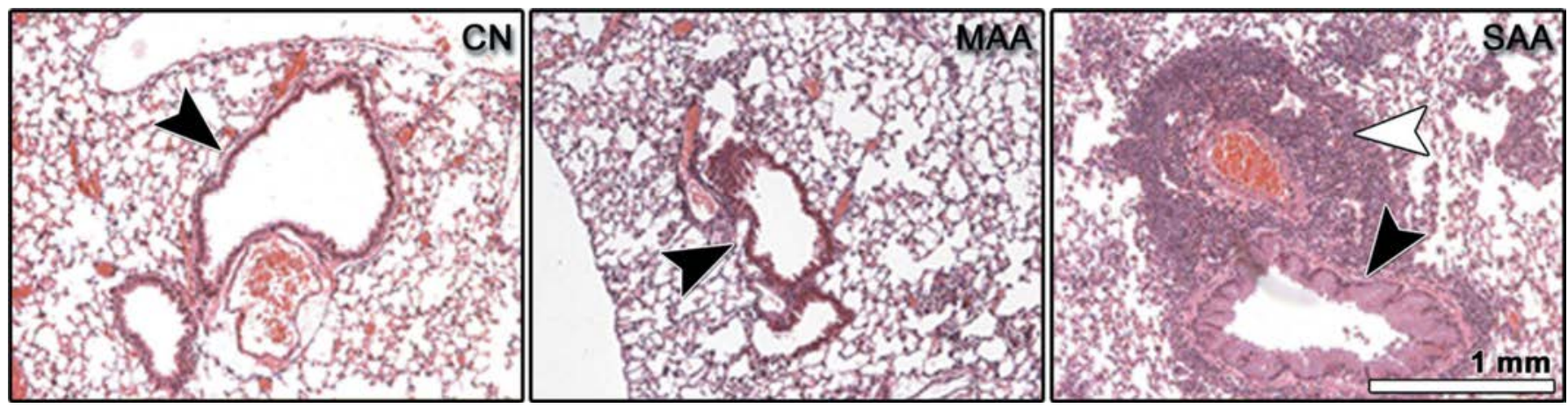

Figure 3

H\&E stained histological sections of a control lung $(\mathrm{CN})$, of a lung from a mouse taken from the mild (MAA) and from the severe (SAA) experimental allergic lung disease model, all sacrificed four days after the last OVA challenge. Lung sections of mice from MAA and SAA show airway wall thickening (bronchial wall indicated by black arrow heads) and lungs of mice with SAA contain areas of high cell density (white arrow head) which are absent in CN and MAA, all parameters known to be typical hallmarks of asthma. 
in these two experimental allergic airway disease models. In order to further analyse these findings, lungs of an additional cohort of mice were explanted and the wet and dry weight of each left lung lobe was measured for each group (CN, MAA, SAA). In Fig. 2(c) the results of this experiment are displayed as the relative difference to the average left lung lobe weight of $\mathrm{CN}$ (wet and dry, respectively). The wet weight reflects both the weight of the water and the cells in the lung, whereas the dry weight reflects the cells only. MAA shows a $14 \%$ and SAA a $98 \%$ larger wet weight than $\mathrm{CN}$, which changes to $21 \%$ and $95 \%$, respectively, after the drying process. This indicates that the $14 \%$ higher wet weight of the lungs from MAA is rather related to the presence of more cells than to a higher water content, since the difference increased when the lungs were dried. In contrast, in SAA the increased weight of $98 \%$ must be related to both the presence of more cells and increased water content, as the relative weight difference to controls changes only marginally. These results indeed suggest that the two allergic airway disease models lead to different tissue compositions of the lung.

To compare these results with the obtained phase-contrast CT $\delta$-values (Fig. $2 b$ ), the soft-tissue volume fraction also has to be considered. MAA shows an about $65 \%$ and SAA an about $175 \%$ increased soft-tissue volume fraction compared with $\mathrm{CN}$. Therefore, the ratio between the increased amount of cells and increased soft-tissue volume fraction is 0.32 in MAA $(21 \% / 65 \%)$ and 0.54 in SAA $(95 \% / 175 \%)$ indicating that the cells in MAA are more loosely packed than in SAA. This effect may cause the different trends in the observed average $\delta$-values in MAA and SAA. The packaging of cells may be related to the extracellular matrix (ECM) and it is known that in asthma an increased percentage of areas of collagen can be found in the ECM (Weitoft et al., 2014), which most likely causes the swelling and reduction in lung tissue elasticity. Therefore, our results indicate a difference in the ECM components between mild and severe asthma, a theory which needs to be studied in more detail.

In summary, we show that synchrotron inline phase-contrast CT with a pixel size of about $9 \mu \mathrm{m} \times 9 \mu \mathrm{m}$ in combination with a single-distance phase-retrieval algorithm provides the necessary image quality for discrimination and quantification of variable alterations in the lung structure. Our results show that, based on quantification of structural alterations within the lung, even mice from a weak allergic airway disease model can be significantly discriminated from controls and stronger allergic airway disease and highlights the robustness and versatility of the presented approach. Inline phase-contrast CT in combination with single-distance phase retrieval therefore represents a valuable tool for the characterization of morphological changes in allergic airway disease mouse models and may aid the analysis of the efficacy of novel therapeutic approaches.

\section{Acknowledgements}

This work was supported by the German Research Foundation (Deutsche Forschungsgemeinschaft, DFG) [DU 1403/1-1] and by the COST MP1207 action. The authors thank Sarah Greco and Bärbel Heidrich for their excellent work of setting up the asthma models used in the study. In addition, we thank the whole team of the SYRMEP beamline which has contributed to this work in many different ways; we especially thank Nicola Sodini for his technical assistance and essential support for the success of the study. Also, we are very grateful to Tim Gureyev for his help using the $X$-tract software, used to perform the phase retrieval, and for some very useful discussions.

\section{References}

Bates, J. H., Rincon, M. \& Irvin, C. G. (2009). Mol. Physiol. 297, L401-L410.

Bayat, S., Porra, L., Suhonen, H., Janosi, T., Strengell, S., Habre, W., Petak, F., Hantos, Z., Suortti, P. \& Sovijärvi, A. (2008). Eur. J. Radiol. 68, S78-S83.

Beltran, M. A., Paganin, D. M., Siu, K. K. W., Fouras, A., Hooper, S. B., Reser, D. H. \& Kitchen, M. J. (2011). Phys. Med. Biol. 56, 7353 7369.

Biffi, S., Dal Monego, S., Dullin, C., Garrovo, C., Bosnjak, B., Licha, K., Welker, P., Epstein, M. M. \& Alves, F. (2013). PLoS One, 8, e57150.

Bosnjak, B., Tilp, C., Tomsic, C., Dekan, G., Pieper, M. P., Erb, K. J. \& Epstein, M. M. (2014). Pulm. Pharmacol. Ther. 27, 44-51.

Busacker, A., Newell, J. D. Jr, Keefe, T., Hoffman, E. A., Cook Granroth, J., Castro, M., Fain, S. \& Wenzel, S. (2009). CHEST J. 135, 48.

Dullin, C., dal Monego, S., Larsson, E., Mohammadi, S., Krenkel, M., Garrovo, C., Biffi, S., Lorenzon, A., Markus, A., Napp, J., Salditt, T., Accardo, A., Alves, F. \& Tromba, G. (2015). J. Synchrotron Rad. 22, 143-155.

Epstein, M. M. (2004). Int Arch Allergy Immunol. 133, 84100.

Gelb, A. F., Licuanan, J., Shinar, C. M. \& Zamel, N. (2002). Chest, 121, $715-721$.

Gureyev, T. E., Mayo, S. C., Myers, D. E., Nesterets, Y., Paganin, D. M., Pogany, A., Stevenson, A. W. \& Wilkins, S. W. (2009). J. Appl. Phys. 105, 102005.

Gureyev, T., Mohammadi, S., Nesterets, Y., Dullin, C. \& Tromba, G. (2013). J. Appl. Phys. 114, 144906.

Hooper, S. B., Kitchen, M. J., Wallace, M. J., Yagi, N., Uesugi, K., Morgan, M. J., Hall, C., Siu, K. K. W., Williams, I. M., Siew, M., Irvine, S. C., Pavlov, K. \& Lewis, R. A. (2007). FASEB J. 21, 3329 3337.

Kitchen, M. J., Lewis, R. A., Yagi, N., Uesugi, K., Paganin, D., Hooper, S. B., Adams, G., Jureczek, S., Singh, J., Christensen, C. R., Hufton, A. P., Hall, C. J., Cheung, K. C. \& Pavlov, K. M. (2005). Br. J. Radiol. 78, 1018-1027.

Kitchen, M. J., Paganin, D., Lewis, R. A., Yagi, N., Uesugi, K. \& Mudie, S. T. (2004). Phys. Med. Biol. 49, 4335-4348.

Lewis, R. (1997). Phys. Med. Biol. 42, 1213-1243.

Lewis, R. A., Yagi, N., Kitchen, M. J., Morgan, M. J., Paganin, D., Siu, K. K. W., Pavlov, K., Williams, I., Uesugi, K., Wallace, M. J., Hall, C. J., Whitley, J. \& Hooper, S. B. (2005). Phys. Med. Biol. 50, 50315040.

Markus, M. A., Dullin, C., Mitkovski, M., Prieschl-Grassauer, E., Epstein, M. M. \& Alves, F. (2014). PLoS One, 9, e90017.

Mohammadi, S., Larsson, E., Alves, F., Dal Monego, S., Biffi, S., Garrovo, C., Lorenzon, A., Tromba, G. \& Dullin, C. (2014). J. Synchrotron Rad. 21, 784-789. 


\section{short communications}

Nabe, T., Zindl, C. L., Jung, Y. W., Stephens, R., Sakamoto, A., Kohno, S., Atkinson, T. P. \& Chaplin, D. D. (2005). Eur. J. Pharmacol. 521, 144-155.

Paganin, D., Mayo, S., Gureyev, T. E., Miller, P. R. \& Wilkins, S. W. (2002). J. Microsc. 206, 33-40.

Parsons, D. W., Morgan, K., Donnelley, M., Fouras, A., Crosbie, J., Williams, I., Boucher, R. C., Uesugi, K., Yagi, N. \& Siu, K. K. W. (2008). J. Anat. 213, 217-227.
Weitoft, M., Andersson, C., Andersson-Sjöland, A., Tufvesson, E., Bjermer, L., Erjefält, J. \& Westergren-Thorsson, G. (2014). Respir. Res. 15, 67.

Yagi, N., Suzuki, Y., Umetani, K., Kohmura, Y. \& Yamasaki, K. (1999). Med. Phys. 26, 2190.

Yong, H. S., Kang, E.-Y., Kim, Y. K., Woo, O. H., Shin, B. K., Oh, C. H., Je, J. H., Han, H. \& Seo, J. S. (2009). Yonsei Med. J. 50, 422426. 
Journal of

Synchrotron

Radiation

ISSN 1600-5775

Received 20 June 2014

Accepted 2 October 2014

\section{Functionalized synchrotron in-line phase-contrast computed tomography: a novel approach for simultaneous quantification of structural alterations and localization of barium-labelled alveolar macrophages within mouse lung samples}

\author{
Christian Dullin, ${ }^{\mathrm{a} *} \neq$ Simeone dal Monego, ${ }^{\mathrm{b}} \neq$ Emanuel Larsson,,${ }^{\mathrm{c}, \mathrm{d}, \mathrm{e}_{\ddagger}}$ \\ Sara Mohammadi, ${ }^{c}$ Martin Krenkel, ${ }^{\mathrm{f}}$ Chiara Garrovo, ${ }^{\mathrm{g}}$ Stefania Biffi, ${ }^{\mathrm{g}}$ \\ Andrea Lorenzon, ${ }^{b}$ Andrea Markus, ${ }^{\text {h Joanna Napp, }}{ }^{\text {h,i,a }}$ Tim Salditt, ${ }^{f}$ \\ Agostino Accardo, ${ }^{d}$ Frauke Alves ${ }^{h, i, a}$ and Giuliana Tromba ${ }^{{ }_{9}}$ \\ anstitute for Diagnostic and Interventional Radiology, University Medical Center Göttingen, \\ Robert Koch Strasse 40, 37075 Göttingen, Germany, ${ }^{\mathbf{b}}$ Cluster in Biomedicine, AREA Science Park \\ Basovizza, Trieste, Italy, ${ }^{\mathbf{C}}$ Elettra Sincrotrone Trieste, Strada Statale 14, km 163.5 in AREA Science \\ Park, 34149 Basovizza (Trieste), Italy, ${ }^{\mathbf{d}}$ Department of Architecture and Engineering, University of \\ Trieste, Trieste, Italy, ${ }^{\mathbf{e}}$ Department of Physics, Chemistry and Biology, Linköping University, SE-581 \\ 83 Linkoeping, Sweden, 'Institute for X-ray Physics, University of Göttingen, Göttingen, Germany, \\ ${ }^{\mathrm{g}}$ Institute for Maternal and Child Health, IRCCS Burlo Garofolo, Trieste, Italy, 'hepartment of \\ Haematology and Medical Oncology, University Medical Center Göttingen, Robert Koch Strasse \\ 40, 37075 Göttingen, Germany, and 'Molecular Biology of Neuronal Signals, Max Planck Institute \\ for Experimental Medicine, Hermann-Rein-Strasse 3, 37075 Göttingen, Germany. \\ *E-mail: christian.dullin@med.uni-goettingen.de
}

Functionalized computed tomography (CT) in combination with labelled cells is virtually non-existent due to the limited sensitivity of X-ray-absorption-based imaging, but would be highly desirable to realise cell tracking studies in entire organisms. In this study we applied in-line free propagation X-ray phasecontrast CT (XPCT) in an allergic asthma mouse model to assess structural changes as well as the biodistribution of barium-labelled macrophages in lung tissue. Alveolar macrophages that were barium-sulfate-loaded and fluorescentlabelled were instilled intratracheally into asthmatic and control mice. Mice were sacrificed after $24 \mathrm{~h}$, lungs were kept in situ, inflated with air and scanned utilizing XPCT at the SYRMEP beamline (Elettra Synchrotron Light Source, Italy). Single-distance phase retrieval was used to generate data sets with ten times greater contrast-to-noise ratio than absorption-based CT (in our setup), thus allowing to depict and quantify structural hallmarks of asthmatic lungs such as reduced air volume, obstruction of airways and increased soft-tissue content. Furthermore, we found a higher concentration as well as a specific accumulation of the barium-labelled macrophages in asthmatic lung tissue. It is believe that XPCT will be beneficial in preclinical asthma research for both the assessment of therapeutic response as well as the analysis of the role of the recruitment of macrophages to inflammatory sites.

Keywords: phase-contrast CT; single-distance phase retrieval; functional CT imaging.

\section{Introduction}

Lung imaging, especially of the mouse, remains extremely challenging due to the small size and high porosity nature of the organ, which creates various problems like scattering for $\mathrm{X}$-ray and optical imaging techniques, susceptibility artefacts

$\ddagger$ These authors contributed equally to this work.

- These authors contributed equally to this work. in magnetic resonance imaging (MRI) and shadowing of medical ultrasound waves at the rib cage. Together with preclinical airway disease models, novel imaging technologies are becoming increasingly important for monitoring disease progression and the efficacy of treatment within the lung. Recent studies have either focused on functional aspects, utilizing near-infrared fluorescence (NIRF) imaging (Napp et al., 2010; Markus et al., 2014) or on the depiction of morpho- 
logical alterations, such as airway wall thickening using ex vivo micro-CT (Cortez-Retamozo et al., 2008; Sera et al., 2007). For tracking of ex vivo labelled immune cells in vivo a wide range of imaging modalities, for example MRI (Ahrens \& Bulte, 2013) and optical imaging (Bousso \& Moreau, 2012), have been reported in recent years. Radionuclide labelling of cells is the oldest technique for tracking immune cells, especially in whole body distribution studies in humans (Thakur, 1977). The application of CT in combination with novel contrast agents for cell tracking has just begun to be explored and is hampered by the poor sensitivity of CT combined with the low toleration of high concentrations of contrast media loaded into cells (Cormode et al., 2014). In order to visualize the biodistribution of labelled cells following injection, as well as to assess morphological alterations in great detail during inflammation and airway remodelling within the lung, the combination of high spatial resolution with increased sensitivity is highly desirable. An imaging technique that meets these requirements is the here-used in-line free-propagation $\mathrm{X}$-ray phase contrast CT (XPCT).

Since the very first application of X-rays for medical purposes by Konrad Roentgen in 1895, the imaging principle for detecting tissue-dependent variations by X-ray absorption has remained unchanged. Especially in applications related to visualization of soft-tissue as in mammography or in lung imaging, these differences in X-ray absorption are very weak, resulting in poor contrast. This contrast could be raised by lowering the X-ray photon energy; however, this would also increase the radiation dose deposition in the samples. Additionally, an energy level high enough to achieve sufficient penetration of the sample needs to be maintained. Due to these factors the absorption contrast in radiographs is limited, especially in clinical practice. In addition to absorption, a phase shift of the incident X-ray wavefront occurs within the sample. Despite being about 100 times stronger in low-density materials (Takeda et al., 1995), this effect has basically not been exploited in clinical routine to date, due to the fact that at least partial coherent X-rays are required, which can only be generated with micro-focus X-ray tubes, or with higher intensities, at synchrotron light sources (Nugent, 2010). This limitation notwithstanding, X-ray phase-contrast imaging has great potential, as it combines strong edge enhancement in radiographs with the fact that the advantage of phase contrast over conventional absorption contrast improves with increasing photon energy. Therefore, medical phase-contrast $\mathrm{X}$-ray imaging could potentially be performed at higher energies than in the actual absorption-based regime, which would reduce the dose deposition within the patient, especially in soft-tissue applications like mammography. There are already some clinical mammography systems on the market demonstrating a gain in image contrast due to phase effects (Tanaka et al., 2005). Besides the here-used in-line freepropagation XPCT, there are other phase-sensitive techniques such as grating interferometry (Pfeiffer et al., 2006). Grating interferometry would allow phase-contrast imaging even when conventional X-ray tubes are used. Notwithstanding that the clinical application of this technique is at the moment hampered by technical problems such as the magnitude of the applied radiation dose, grating interferometry also showed very promising results in lung imaging as demonstrated by Schleede et al. (2012).

Since absorption-based CT imaging in its nature shows low sensitivity, functional imaging approaches similar to those used in SPECT, PET or optical imaging (Nahrendorf et al., 2008) are virtually nonexistent. The potential of phase-sensitive techniques for lung imaging has been explored since the late 1990s at some synchrotron beamlines (Yagi et al., 1999; Kitchen et al., 2004; Lewis et al., 2005; Hooper et al., 2007, 2009). However, the increased sensitivity of XPCT in less dense materials might be well suited to realise CT functional imaging addressing both soft-tissue alterations and the distribution of heavy-ion-based contrast agents such as barium sulfate. Contrary to classical absorption-based CT, in XPCT edge enhancement caused by phase effects at tissue interfaces is superimposed on the contrast caused by tissue-specific differences in X-ray absorption. These interactions of X-rays with matter are described by the complex refractive index $\tilde{n}=1-\delta+i \beta$, where $\delta$ determines the phase shift and $\beta$ the absorption. In order to analyse the phase shift separately, the $\delta$ part of the signal needs to be decoupled by a phase-retrieval $(\mathrm{PhR})$ algorithm. It has already been demonstrated that the application of $\mathrm{PhR}$ is highly beneficial in the analysis of biological samples (Keyriläinen et al., 2010; Zhang et al., 2011; Yong et al., 2009; Gureyev et al., 2013) showing an up to 200fold improvement in the contrast-to-noise ratio (Beltran et al., 2011). A large variety of $\mathrm{PhR}$ algorithms have been reported so far, that can be loosely divided into multi- and singledistance techniques (Nugent, 2007).

Commonly used multi-distance $\mathrm{PhR}$ approaches are either based on the fact that the absorption part of the detected radiograph is constant whereas the impact of the phase effects varies with different sample-to-detector distances, or require a priori knowledge of the refractive indexes within the sample (Cloetens et al., 1999a; Beltran et al., 2010). Imaging with several sample-to-detector distances is disadvantageous because it crucially depends on a perfect alignment of the different scans and it is affected by variations in the X-ray beam, which often occur at synchrotron light sources. In contrast to that, single-distance $\mathrm{PhR}$ algorithms minimize the scanning time and are therefore especially suitable for the analysis of unfixed biological samples such as in situ mouse lungs imaged in this study, but they can strictly only be applied on objects expressing a constant $\delta$-to- $\beta$ ratio (Paganin et al., 2002). However, the applicability of this type of algorithm for soft-tissue samples was shown by Wu et al. (2005). Moreover, single-distance $\mathrm{PhR}$ followed by standard filtered backprojection reconstruction (FBP) was applied to generate three-dimensional (3D) data sets of mouse lungs predominately presenting the distribution of the $\delta$ part of the refractive index (Mohammadi et al., 2014).

The aim of this study was to develop a functional XPCT imaging approach (fXPCT) by exploiting the capability of alveolar macrophages $(M \Phi)$ to migrate to inflammatory sites within the lung using an ovalbumin induced experimental 
allergic airways disease model (Markus et al., 2014). In order to visualize the biodistribution of cells following intratracheal application, $\mathrm{M} \Phi$ of the immortalized alveolar macrophage cell line MH-S (Mbawuike \& Herscowitz, 1989) were ex vivo labelled by adding a contrast agent suitable for X-ray-based imaging directly to the cell culture media. A barium sulfate suspension was used, that is commonly applied in the clinic to mark the gastrointestinal tract in CT (Golder et al., 1991). Thereby, macrophages were intracellularly labelled and used as a specific probe instead of an in situ labelling approach by systemic administration of X-ray contrast agents. $M \Phi$ can engulf large particles by phagocytosis and can therefore easily be loaded with contrast agents and drugs in vitro (Trivedi et al., 2006), converting them into potential carriers for both diagnostic and therapeutic agents. Since they migrate to inflammatory sites they have already been exploited for delivery of various anti-inflammatory compounds (Bang et al., 2011; Moreira \& Hogaboam, 2011; Yang et al., 2012). Moreover, MФ were recently identified as one of the main effector cells in asthma (Bang et al., 2011; Moreira \& Hogaboam, 2011; Yang et al., 2012). Mizue et al. (2005) showed that, in the absence of the macrophage migration inhibitory factor (MIF), asthma could not be induced in MIF-deficient mice, and Chen et al. (2010a) demonstrated in an asthma mouse model that airway remodelling was successfully inhibited by a MIF antagonist.

With the unique capabilities of XPCT in combination with phase retrieval and the use of $M \Phi$ loaded with barium sulfate particles, we were able to simultaneously depict and quantify structural features and to illustrate in 3D the different accumulation sites of labelled $\mathrm{M} \Phi$ within asthmatic and control lungs. We believe that, by providing new quantitative functional and anatomical parameters and by using bariumlabelled immune cells in cell trafficking studies, this novel fXPCT approach may help to preclinically investigate complex and multi-factorial processes of inflammatory diseases.

\section{Material and methods}

\subsection{Preparation and labelling of macrophages}

The immortalized mouse alveolar $\mathrm{M} \Phi$ cell line MH-S (purchased from American Type Culture Collection, ATCC, USA) was maintained in RPMI medium, supplemented with $10 \%$ FCS and $0.05 \mathrm{mM}$ 2-mercaptoethanol (Mbawuike \& Herscowitz, 1989) in a humidified atmosphere at $5 \% \mathrm{CO}_{2}$ and $310 \mathrm{~K}$. For CT imaging, cells were loaded with a barium sulfate suspension, the clinically used contrast agent Micropaque CT (Guerbet, France) by co-incubating $1 \times 10^{6}$ cells ml $^{-1}$ for $24 \mathrm{~h}$ with $3.5 \mu \mathrm{l}$ Micropaque $\mathrm{CT} / \mathrm{ml}$ cell media $(175 \mu \mathrm{g}$ barium sulfate/ml media), followed by two washing steps with phosphate buffer (PBS). Subsequently, for stable fluorescent labelling of the cell membrane, the $\mathrm{M} \Phi$ were incubated for 30 min with $5 \mu \mathrm{ml}^{-1}$ of the lipophilic dialkylcarbocyanine dye Vibrant DiD (DiD; Molecular Probes, Eugene, OR, USA; excitation maximum: $644 \mathrm{~nm}$; emission maximum: $665 \mathrm{~nm}$ ), followed by two washing steps with PBS. To test the loading efficacy of barium sulfate particles into $M \Phi$, a vial containing
$1 \times 10^{5}$ barium sulfate $\mathrm{M} \Phi$ resuspended in $100 \mu \mathrm{l}$ PBS was scanned with a normal bench-top microCT (eXplore locus SP, GE HealthCare, USA) (Verdelis et al., 2011). The morphology of the barium-labelled $M \Phi$ was assessed by light microscopy and the effects of the uptake of barium sulfate particles on the metabolic activity of $\mathrm{M} \Phi$ were investigated with a watersoluble tetrazolium (WST-1) cell proliferation assay (Madison, WI, USA) (Mosmann, 1983).

\subsection{Mouse model of allergic asthma}

Female BALB/c mice (4-6 weeks old) were purchased from Harlan Laboratories and maintained with 'ad libitum' food and water. For generation of the experimental allergic airways disease model, mice were sensitized intraperitoneally (i.p.) at day 0 and 21 with $10 \mu \mathrm{g}$ ovalbumin (OVA), dissolved in $200 \mu \mathrm{l}$ PBS. At day 28 and 29, mice were treated intranasally (i.n.) with a solution of $100 \mu \mathrm{g}$ OVA/50 $\mu \mathrm{l}$ PBS to induce an acute allergic reaction (Biffi et al., 2013). Healthy age and gendermatched BALB/c mice, immunized and challenged with PBS only, were used as controls. To verify the success of OVA immunization, blood samples were taken $72 \mathrm{~h}$ after the last challenge from the facial vein of the living mice and levels of OVA-specific immunoglobulin within the sera were analysed as previously described (Biffi et al., 2013).

Animal in vivo procedures were performed at the CBM Animal Facility, Trieste, Italy, in compliance with the guidelines of the European (86/609/EEC), the Italian (DL116/92) and at the University Medical Center Göttingen, Germany, in accordance with the German ethical laws (33.9-42502-04-10/ 0134) and were approved by the Italian Ministry of Health as well as by the animal ethics administration of Lower Saxony, Germany.

\subsection{Application of macrophages and experimental setup}

Previous differential cell counts from bronchoalveolar lavages (BAL) and in vivo fluorescence measurement experiments showed that OVA-induced asthmatic mice display the strongest signs of inflammation between $48 \mathrm{~h}$ and $72 \mathrm{~h}$ after the last antigen challenge (Biffi et al., 2013). We therefore instilled $6 \times 10^{6}$ barium- and DiD-labelled M $\Phi$ resuspended in $30 \mu \mathrm{l}$ PBS intratracheally (i.t.) $72 \mathrm{~h}$ after the last OVA challenge into the lungs of asthmatic and control mice under xylazine-tiletamine-zolazepam anaesthesia.

\subsection{In vivo optical imaging}

Optical imaging was performed by two-dimensional fluorescence reflectance imaging (FRI) using the Optix MX2 system (ART; Montreal, Canada) as previously described (Markus et al., 2014). For in vivo scans, mice were anaesthetized by inhalation with isoflurane ( $2 \%$ isoflurane in 21 oxygen per min). Before imaging, mice were shaved and chemically depilated over thorax and abdomen to decrease scattering from the fur. Mice were scanned before, directly after and $24 \mathrm{~h}$ after $M \Phi$ instillation. All data were acquired using a $670 \mathrm{~nm}$ excitation laser diode in combination with a $700 \mathrm{Lp}$ emission filter and a $1.0 \mathrm{~mm}$ raster. The fluorescence 
intensity was analysed using the OptiView-2-02-00 software (ART). The average intensity of the lung area was measured and its relative increase compared with the pre-scan was computed.

\subsection{Preparation of biological samples for ex vivo $\mathrm{CT}$ analysis}

Mice were sacrificed $24 \mathrm{~h}$ after instillation of bariumsulfate-loaded and fluorescent-labelled M $\Phi$ (Fig. 1) using a xylazine-tiletamine-zolazepam overdose. In order to ensure comparability between different samples, all lungs were inflated in situ with air, under a constant pressure of $30 \mathrm{~cm}$ water column $(2.94 \mathrm{kPa})$ through a series of smaller tubes, which ended in a PE50 polyethylene cannula fixed inside the trachea with a cotton thread. Tracheas were tied up and all samples were kept at room temperature for $2 \mathrm{~h}$, in order to avoid any alterations caused by rigor mortis. To avoid air leakage, alterations and movements during the X-ray examination, samples were then embedded in a $1 \%$ agarose gel inside $30 \mathrm{ml}$ tubes (Fisher Scientific, USA) and kept for $30 \mathrm{~min}$ at $277 \mathrm{~K}$. Samples were moved to the synchrotron beamline $30 \mathrm{~min}$ before scanning to allow for temperature adaptation. Four lung samples were prepared for fXPCT analysis: one mouse with OVA-induced asthma and two healthy controls, all injected with $\mathrm{M} \Phi$ (AA, CN1 and CN2), as well as one healthy mouse without application of $\mathrm{M} \Phi$ which served as a negative control (Blk).

\subsection{Synchrotron-radiation-based $\mathrm{fXPCT}$ and phase retrieval}

All data sets were acquired at the SYRMEP beamline at the synchrotron light source Elettra (Trieste, Italy) (Brun et al., 2010; Dreossi et al., 2008), which is especially designed for medical applications and analysis of biological samples. The beamline was operated at $22 \mathrm{keV}$ at a sample-to-detector distance of $30 \mathrm{~cm}$. The central area of the lung of each sample was scanned by performing two overlapping $360^{\circ}$ scans with 1800 projections each and a $2 \times 2$ binning of the detector

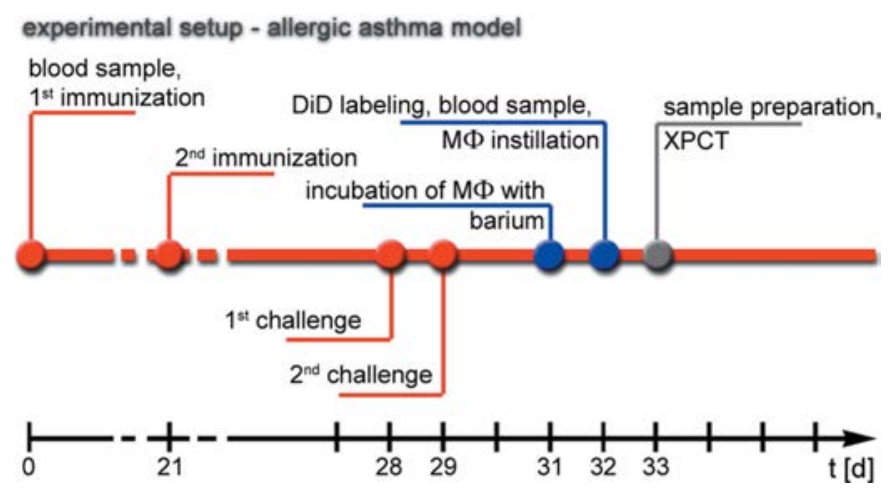

Figure 1

Experimental setup. An OVA allergen-induced experimental allergic airways disease model was used, consisting of two immunization steps (i.p. injection of $10 \mu \mathrm{g}$ OVA) on days 0 and 21 and two challenging steps (i.n. application of $100 \mu \mathrm{g}$ OVA) on days 28 and 29. Prior to their instillation, $\mathrm{M} \Phi$ were labelled with Micropaque and DiD for 24 and $4 \mathrm{~h}$, respectively. On day 32, $6 \times 10^{6}$ barium- and DiD-labelled M $\Phi$ were instilled i.t. into asthmatic and control mice. Mice were euthanized on day 33 and prepared for fXPCT analysis. elements, thus resulting in a spatial resolution of $9 \mu \mathrm{m}$. In order to decouple phase and absorption information in the acquired projection images, we applied a single-distance inline phase-retrieval algorithm based on the Born equation implemented by Chen et al., which only requires one data set, obtained at a single sample-to-detector distance (Mohammadi et al., 2014; Chen et al., 2010b, 2012; Taylor, 1981). In order to apply this class of algorithms, a priori knowledge of the $\delta$-to- $\beta$ ratio within the sample is needed. Within this study we used a $\delta$-to- $\beta$ ratio of 1950 for standardized lung tissue with a hydrogen, carbon and oxygen ratio: H10 C0.83 O5 (International Commission on Radiological Protection) (Mohammadi et al., 2014).

\subsection{Quantification of morphological alterations within the lung and distribution of barium-loaded macrophages}

For a quantitative comparison of the lung samples it is crucial to identify parameters that characterize the alterations in the lung structure without being affected by local inhomogeneities in the manifestation of the asthmatic reaction within the lung. To this end we applied an analysis scheme that was adapted from strategies used for the characterization of trabecular bone structure and other porous materials. Briefly, the data sets were reoriented and resampled to ensure that the analysis is independent of the orientation of the lungs during the scan. Due to the high contrast-to-noise ratio in the phaseretrieved reconstructed data sets, non-overlapping greyscale ranges were assigned to air, lung soft-tissue, bone of the rib cage and to barium used as label for the i.t. instilled MФ. These image segments were then further analysed and quantified in terms of air, soft-tissue and barium content as well as narrowing of the airways.

\subsection{Histology}

Lung samples for histology were obtained from a further set of OVA-induced asthmatic and control mice following the schedule and conditions as described in Fig. 1 (in order to be comparable with the fXPCT analysis). The excised lungs were fixed in $10 \%$ buffered formalin and embedded in paraffin, and $3 \mu \mathrm{m}$-thick paraffin lung sections containing main stem bronchi were obtained. A periodic acid-Schiff (PAS) staining was performed to assess bronchial wall thickness and mucus production (Fullmer, 1960). Sections were deparaffinised, rehydrated and stained with periodic acid for $5 \mathrm{~min}$, followed by Schiffs reagent (Merck, Darmstadt, Germany) for $15 \mathrm{~min}$ and hematoxylin for $2 \mathrm{~min}$. Slices were washed for $3 \mathrm{~min}$ between each of the staining steps. The samples were dehydrated using an ascending alcohol series and Xylol and finally mounted with DePex (Serva, Heidelberg, Germany). An Axioskop 2 (Carl Zeiss Microscopy GmbH, Jena, Germany) microscope in combination with a Leica DC 100 camera (Leica, Switzerland) was used for analysis of the stained sections. 


\subsection{Fluorescence microscopy}

In order to verify the location of the injected DiD and barium-labelled $M \Phi$, fluorescence microscopy of lung tissue sections of asthmatic and control mice was performed, utilizing an Axiovert 200M inverted microscope (Carl Zeiss Microscopy) equipped with a xenon lamp and a high-sensitivity ORCA-AG digital camera (Hamamatsu, Japan), capable of NIRF detection. For this purpose, lungs of asthmatic mice $(n=2)$ and a control mouse, explanted $24 \mathrm{~h}$ after M $\Phi$ i.t. instillation, were filled with optimal-cutting-temperature (OCT) embedding material (Tissue-Tek; Sakura Finetek, Torrance, CA, USA) and were cryofrozen. Lung cryosections $(5 \mu \mathrm{m})$ were obtained, fixed with acetone $(10 \mathrm{~min}$ at $253 \mathrm{~K})$ and washed with tris(hydroxymethyl)-aminomethane (Tris) buffer ( $\mathrm{pH}$ 7.5). Immunostaining was performed as follows. Autofluorescence was blocked with $0.1 \mathrm{M}$ glycin [for $10 \mathrm{~min}$ at room temperature (RT)], endogenous biotin and avidin were blocked with Avidin Biotin Blocking Solution and unspecific binding sites for $20 \mathrm{~min}$ at RT with SEA BLOCK blocking buffer (both Thermo Scientific) following the manufacturer's protocol. Slices were then incubated overnight at $277 \mathrm{~K}$ with rat monoclonal anti-CD68 antibody (FA-11; Abcam, Cambridge, UK; $3.33 \mu \mathrm{g} \mathrm{ml}^{-1}$ ) diluted in Antibody Diluent with Background Reducing Components (DAKO, Glostrup, Denmark), followed by two incubation steps of $1 \mathrm{~h}$ at RT with biotinylated goat-anti-rat antibody (BioLegend, San Diego, CA, USA; 1:200), and with Streptavidin-AlexaFluor 555 (Molecular Probes, Life Technologies, Carlsbad, CA, USA; 1:400). Finally, slices were mounted with Mowiol (Calibiochem, Merck, Darmstadt, Germany) supplemented with 4',6-diamidino-2-phenylindole (DAPI) for staining of the nuclei and left overnight at $277 \mathrm{~K}$. Two washing steps with Tris buffer were performed between each step. DiD fluorescence was acquired using a band-pass (BP) $640 \pm 15 \mathrm{~nm}$ excitation filter, a $660 \mathrm{~nm}$ dichroic mirror and a BP $690 \pm 25 \mathrm{~nm}$ emission filter. The AlexaFluor 555 signals were recorded using a BP $546 \pm 6 \mathrm{~nm}$ excitation filter, a $580 \mathrm{~nm}$ dichroic mirror and a $590 \mathrm{~nm}$ long pass emission filter. DAPI fluorescence was acquired using a BP $365 \pm 12.5 \mathrm{~nm}$ excitation filter, a $395 \mathrm{~nm}$ dichroic mirror and a BP $445 \pm 25 \mathrm{~nm}$ emission filter. In the produced images the DAPI channel was set to blue, AlexaFluor 555 to green and DiD to red. Image generation and processing were performed using the software AxioVision Rel.4.6 (Carl Zeiss Microscopy GmbH, Jena, Germany) and ImageJ (National Institutes of Health, Bethesda, MD, USA) (Collins, 2007).

\subsection{High-resolution synchrotron-radiation-based X-ray phase-contrast microCT (HR microCT)}

Lung sections of a third set of OVA-induced asthmatic mice $(n=2)$ and a control mouse following the schedule and conditions as described in Fig. 1 were also studied using a CT set-up with higher resolution. For this aim the procedure for sample preparation was the following: the trachea was cannulated, then filled and fixed with $4 \%$ paraformaldehyde (PFA), and subsequently single lobes of the lung were embedded in a $5 \%$ agarose-gel. Finally, $500 \mu \mathrm{m}$-thick slices were cut using a Vibratome (Leica VT 1000S; Leica, Switzerland). The slices were placed with a droplet of PBS between two round pieces of polypropylene foil, secured within aluminium rings. These were glued together to create a closed chamber for the slices (Olendrowitz et al., 2012). These slices were then imaged with a divergent X-ray beam of $17.5 \mathrm{keV}$ at the beamline ID22 $\mathrm{Ni}$ at the European Synchrotron Radiation Facility (ESRF) in Grenoble. The beam was focused to less than $100 \mathrm{~nm} \times 100 \mathrm{~nm}$ by Kirkpatrick-Baez (KB) mirrors and free propagation phase-contrast images recorded with a FReLoN camera (Analog and Transient Electronic ESRF group) coupled to a scintillator (Weitkamp et al., 1999). The sample was placed at the maximum possible defocus distance to achieve the highest field of view. Using a ten-fold objective lens behind the scintillator and the magnification of the KB beam an effective pixel size of $430 \mathrm{~nm}$ was achieved. Tomographic scans with a series of 1500 images over a full rotation of $360^{\circ}$ were recorded for several specimens of asthma and control mice. Phase retrieval was performed using the single-distance Holo-tomo reconstruction algorithm (Cloetens et al., 1999) implemented at the beamline. Before $3 \mathrm{D}$ reconstruction, each projection image was corrected by an image of the empty beam and aligned to the other projections before phase retrieval was performed.

\subsection{Analysis and statistics}

The 3D rendering of the data sets was performed with VGStudio Max 2.2 (Volume Graphics, Heidelberg, Germany). For mask generation, IDL 7.0 (Research Systems; Boulder, $\mathrm{CO}$, USA) and ImageJ (National Institutes of Health; Bethesda, MD, USA) were used (Abràmoff et al., 2004). Pore3D, a proprietary software library developed by the SYRMEP group, was applied to analyse the 3D barium distribution as well as to quantify the air and tissue content of the lung within volumes of interest (VOIs) (Brun et al., 2010). Statistical analysis was performed using MINITAB (Minitab Ltd; Coventry, UK) and utilizing a one-way ANOVA test with Tukey $90 \%$ simultaneous confidence intervals for the computed parameters (Ryan et al., 2005; Dunn, 1961).

\section{Results}

\subsection{XPCT in combination with single-distance phase retrieval is a valuable tool for lung imaging}

In order to evaluate the usefulness of phase retrieval in our experiment, we performed two scans of a mouse lung sample in XPCT at sample-to-detector distances of 7 and $30 \mathrm{~cm}$. At the distance of $7 \mathrm{~cm}$ only minor edge effects were present and therefore this essentially resembles the absorption-based setup in classical CT, despite the fact that the scan was performed with a monochromatic X-ray source. In contrast, scanning the same sample at a sample-to-detector distance of $30 \mathrm{~cm}$ provides sufficient phase contrast. Phase retrieval was applied to the projection images of the second scan before $3 \mathrm{D}$ reconstruction with FBP was performed. This procedure 
matches the same scheme used in this study. As a basis for a quantitative comparison, we calculated the contrast-to-noise ratio (CNR) using the following equation:

$$
\mathrm{CNR}=\frac{\overline{g_{1}}-\overline{g_{2}}}{\left[\left(\sigma_{1}^{2}+\sigma_{2}^{2}\right) / 2\right]^{1 / 2}},
$$

where $\overline{g_{1}}$ and $\overline{g_{2}}$ denote the mean grey value of two adjoining tissues and $\sigma_{1}^{2}$ and $\sigma_{2}^{2}$ reflect their noise level, measured as squared standard deviation in a region of interest (ROI) (Mohammadi et al., 2014). In each sample and on five reconstructed slices homogeneously distributed over the whole lung, eight circular two-dimensional ROIs (size $\sim 0.4 \mathrm{~mm}^{2}$ ) solely containing either air or lung soft-tissue were defined and analysed. We determined a CNR between air and lungtissue of about 20.0 in the phase-retrieved data set at $30 \mathrm{~cm}$ and 1.9 at $7 \mathrm{~cm}$ without PhR. In addition, only minor blurring of the phase-retrieved images was found (data not shown). These results demonstrate that the combination of XPCT with single-distance phase retrieval is able to increase the softtissue contrast in our samples and setup by a factor of 9.8 when compared with absorption-based monochromatic X-ray imaging. This directly translates into an increased sensitivity that is beneficial for the combined functional and structural CT imaging approach to visualize mouse lung tissue.

\subsection{Macrophages as specific contrast agent for functional CT}

The barium sulfate uptake efficacy of $M \Phi$ was assessed with a bench-top microCT. Imaging of a vial, containing $1 \times 10^{5}$ $\mathrm{M} \Phi$ loaded with barium sulfate particles and resuspended in $100 \mu \mathrm{l}$ PBS, showed a $10 \%$ increase of the X-ray absorption when compared with a vial containing $1 \times 10^{5}$ unlabelled $\mathrm{M} \Phi$ as control (data not shown). The WST-1 assay revealed no influences of the phagocytized barium sulfate particles on the metabolic activity of the M $\Phi$ (data not shown). Furthermore, no evidence of morphologic alterations was observed in barium-sulfate-loaded $\mathrm{M} \Phi$ by light microscopy (data not shown). In conclusion, the approach to load $M \Phi$ with barium provides sufficient contrast for $\mathrm{CT}$ imaging and shows no signs of acute cell toxicity.

\subsection{In vivo optical imaging demonstrates successful intra- tracheal instillation of $M \Phi$}

In vivo NIRF imaging was performed in order to confirm the successful i.t. instillation of the $M \Phi$. For this purpose the $M \Phi$ were additionally stained with the NIR cell membrane label DiD. Fig. 2 shows the in vivo optical imaging results for the same asthmatic mouse analysed later by fXPCT. A strong increase in fluorescence intensity was observed over the lung area directly and $24 \mathrm{~h}$ after i.t. instillation of DiD- and bariumlabelled M $\Phi$ when compared with the pre-scan. These results verify the presence of the labelled $M \Phi$ in the lung area of the mouse $24 \mathrm{~h}$ after i.t. administration.

\subsection{Processing and quantification of FXPCT data sets}

All lungs were kept in situ and scanned at the SYRMEP beamline using a setup that allows for fXPCT. In order to cover the main central area of the lung, two slightly overlapping scans per sample were performed. The original projection data sets were then processed by the single-distance in-line phase-retrieval algorithm (Chen et al., 2010) to create projections predominately showing the $\delta$ part of the complex refractive index. These data sets were later on reconstructed with FBP. In order to analyse the data quantitatively, the following steps were performed: stitching of the two overlapping scans and reorientation of the data sets to allow for comparison between different samples; masking of the lung to restrict the analysis to the lung area; segmentation of the three different components (air, lung soft-tissue and barium); 3D quantification of structural alterations and depiction of the barium concentration and distribution.

\section{Figure 2}
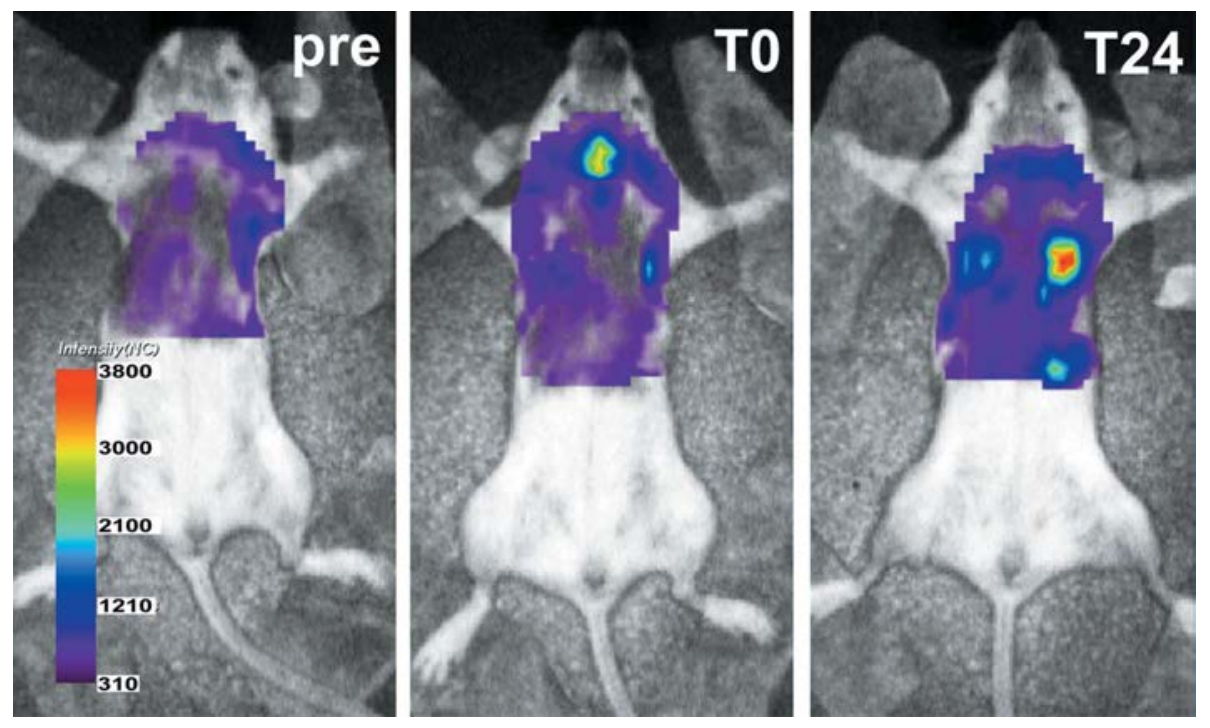

In vivo optical imaging results. Fluorescence intensity maps of the same asthmatic mouse used for fXPCT (Fig. 3) before (pre), directly after (T0) and $24 \mathrm{~h}$ after (T24) i.t. instillation of $6 \times 10^{6}$ barium-sulfate-filled and DiD-labelled M $\Phi$ are shown. After $24 \mathrm{~h}$, strong fluorescence signals expressed in normalized counts (NC) are visible over the lung area. 


\subsubsection{Post-processing steps and descriptive comparison of} the obtained XXPCT lung data sets. The automatic quantification of anatomical alterations is based on the splitting of the data sets into rectangular VOIs. Thus, to ensure that all data sets are present in the same orientation, the reconstructed data of the two scanning steps were registered, combined and re-orientated. In consideration of the memory limitations in the post-processing algorithms, data sets were re-sampled down to an isotropic resolution of $14.4 \mu \mathrm{m}$. A volumerendering representation of the final data sets is shown in Fig. 3(a) and VOIs in Figs. 3(b) and 3(c). To visualize the various tissues as well as the i.t. instilled barium-sulfate-loaded $\mathrm{M} \Phi$, pseudo-colours were assigned to different greyscale ranges. As a result, lung soft-tissue is displayed in red, bones in grey and highly dense areas related to the barium-containing $\mathrm{M} \Phi$ appear yellow. In order to maintain the 3D visibility of the inner lung structure the air was set to transparent. Given that barium causes a greater phase shift than lung soft-tissue and the healthy blank (Blk) contains no barium, the upper limit of the grey value range representing the lung soft-tissue in Blk was chosen as a valuable threshold to detect the bariumloaded MФ.
The segmented images clearly show an increased soft-tissue content in sample AA in comparison with the healthy controls [Figs. 3(a) and 3(b)]. Additionally, the VOI of AA (Fig. 3b) displays a reduced porosity, which illustrates the airway obstruction characteristic for asthma. The same effect of increased soft-tissue content and airway obstruction can to a certain extent also be seen in the planar slices in Figs. 4(a) and 4(b) (white arrow heads). Furthermore, Fig. 3 demonstrates that the barium-sulfate-loaded $M \Phi$ seem to be distributed in cluster-like structures throughout the asthmatic lung, which is also demonstrated in planar reconstructed slices in Figs. 4(c) and $4(d)$. High-contrast regions related to barium-sulfateloaded $M \Phi$ are also present in the lungs of the two healthy controls $\mathrm{CN} 1$ and $\mathrm{CN} 2$; however, at a much lower quantity (yellow spots, Fig. $3 b$ ). Note that $\mathrm{M} \Phi$ derived signals within the lung in AA appear to be surrounded by lung soft-tissue and therefore seem to be originating from areas around the bronchial walls and not from the lumen of the airways (Fig. 3b). The same location of $\mathrm{M} \Phi$ was also found in twodimensional slices as shown in Fig. 4(c). In the same slices areas can be found that are solely composed of soft tissue that is characterized by a lower contrast than the marked spots

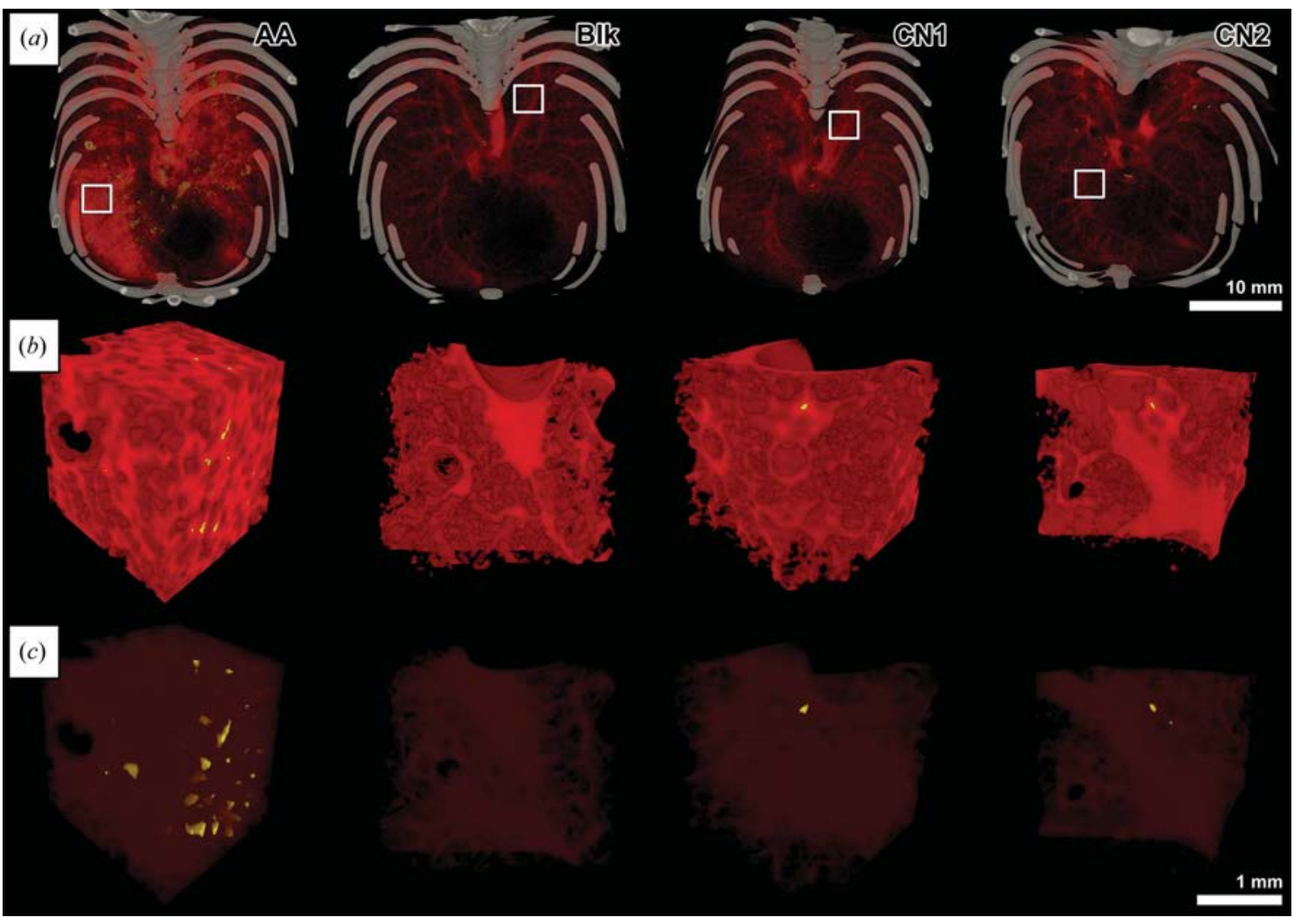

Figure 3

Volume rendering of the fXPCT results and visualization of the MФ localization. Volume renderings of four 3D reconstructions of phase-retrieved fXPCT data sets are shown. Each column represents one lung sample of an asthmatic mouse (AA), a healthy mouse without instilled M $\Phi$ as negative control (Blk), and two healthy controls $(\mathrm{CN} 1, \mathrm{CN} 2)$. Bone is displayed in grey, soft-tissue in red and barium in yellow (air is not depicted). ( $a$ ) Illustration of the entire field of view: more barium and an increased soft-tissue density is shown in AA. (b) VOIs (their origin is indicated by white squares in row 1 ). (c) The same VOIs are shown but with higher transparency to allow visualization of the sites where barium-filled $M \Phi$ cluster in more detail. Note that in the VOIs of the AA lung a higher barium content is visible compared with the results obtained in lungs of the negative control Blk and the healthy controls CN1 and CN2. Additionally, the VOIs of the AA sample show a higher soft-tissue content in comparison with the VOIs of all controls, reflecting a reduced pulmonary volume and increased soft-tissue content in the asthmatic lung tissue. 
assigned to barium. Therefore, it can be excluded that the detected spots are caused by partial-volume effects. Due to the fact that no other strong absorbing material is present in the lung, these high-dense spots most likely represent the instilled barium-labelled $M \Phi$. Therefore, the detected localization of the $M \Phi$ within the bronchial walls may indicate an active migration of the instilled $M \Phi$ from the airspace into the tissue.

3.4.2. Development of an image-processing scheme for automatic 3D quantification of morphological alterations. Since lung tissue has a tree-like structure of airways with different capillarity, the depiction of the VOIs are of crucial importance for a VOI-based analysis scheme. On the other hand, the instilled barium-labelled $M \Phi$ produce a weak signal and might have an inhomogeneous distribution in such a way that they could be easily missed in an approach focused on the entire lung. In order to perform a quantitative comparison, entire lungs were therefore subdivided into three sets of $2 \mathrm{~mm}$-thick bands in the horizontal, vertical and frontal direction. In the so-generated 32 VOIs per sample, two types of parameters were analysed: (i) volume ratio (Vol. Ratio), which defines the volume fraction of a tissue or material of interest compared with the total volume of a VOI, and (ii) structural thickness (St. Th.) of either the airways or the surrounding tissue. Structural thickness is calculated by analysing the maximal size of spheres which can be inscribed in the structure as proposed by Hildebrand \& Ruegsegger (1997) and implemented in the software Pore3D (Brun et al., 2010). These two parameters are directly related to airway wall thickening and airway obstruction and therefore reflect structural changes characteristic for asthmatic lung tissue.

3.4.3. Quantification of morphological changes in the lung. In addition to visual inspection of the rendered fXPCT data sets, quantification of structural changes was a further aim of the study. For this purpose the average and standard variation of the parameters Vol. Ratio and St. Th. were calculated in each sample and in each of the horizontal, vertical and transvertical sets of VOIs, respectively (Table 1). We found an approximately $17 \%$ reduced air content as well as a $61 \%$ increase in the soft-tissue Vol. Ratio in the AA sample compared with all controls (Table 1). In addition, airway obstruction as a hallmark of asthma is clearly reflected in the results of the St. Th. measurements showing a $32 \%$ St. Th. reduction of the mean airway thickness in AA compared with all healthy animals and, vice versa, a $48 \%$ higher St. Th. in the lung softtissue (Table 1).

In order to prove whether the results obtained in the individual VOIs of each data set are significantly different in between the samples, we used a oneway ANOVA test with Tukey $90 \%$ simultaneous confidence intervals to test the difference of the mean values of the analysed parameters (Vol. Ratio of air and soft-tissue and St. Th. of airways and tissue) (Ryan et al., 2005). The comparison of AA and Blk revealed reliable differences for all analysed parameters. Even the minor differences of these parameters between Blk, CN1 and $\mathrm{CN} 2$ were found to be statistically significant, demonstrating the sensitivity of our fXPCT approach. Therefore, based on the chosen parameters (air and soft-tissue volume ratio and mean airway and soft-tissue thickness), the asthmatic and the three control lung samples can be successfully distinguished, indicating that this parameter set can be used to preclinically monitor structural changes in asthmatic lungs. 
Table 1

Mean values \pm standard deviation of the considered parameters.

The pooled standard deviations for the Vol. Ratio (\%) parameter were \pm 0.04 for barium, \pm 4.95 for alveoli and 4.94 for tissue. For the St. Th. $(\mu \mathrm{m})$ parameter the pooled standard deviation were \pm 68 for alveoli and \pm 11 for soft tissue.

\begin{tabular}{lllll}
\hline & Sample & Barium & Air & Soft tissue \\
\hline Vol. Ratio (\%) & AA & $0.10 \pm 0.07$ & $64.62 \pm 6.78$ & $35.28 \pm 6.75$ \\
& Blk & $0.00 \pm 0.00$ & $78.32 \pm 3.43$ & $21.68 \pm 3.43$ \\
& CN1 & $0.00 \pm 0.00$ & $74.28 \pm 4.75$ & $25.72 \pm 4.75$ \\
& CN2 & $0.00 \pm 0.00$ & $81.85 \pm 4.21$ & $18.15 \pm 4.21$ \\
St. Th. $(\mu \mathrm{m})$ & AA & & $188 \pm 39$ & $83 \pm 15$ \\
& Blk & & $254 \pm 53$ & $54 \pm 9$ \\
& CN1 & & $256 \pm 83$ & $60 \pm 9$ \\
& CN2 & & $315 \pm 87$ & $53 \pm 7$ \\
\hline
\end{tabular}

In addition, the histogram of the $\delta$ distribution within the samples, which was normalized to the total amount of analysed voxels for each mouse, showed a more dominant interval in AA (grey values 45-55, data not shown). This result suggests that during the course of asthma not only structural but also changes in the composition of the lung tissue occur, which may be the result of the presence of oedema, an increased mucus production or alterations in the collagen fibres within the lung.

3.4.4. Quantitative analysis of the barium concentration within the lungs. As shown in Fig. 3 (in yellow), barium-filled $\mathrm{M} \Phi$ can be detected in AA and to a lower extent in $\mathrm{CN} 1$ and $\mathrm{CN} 2$. Following the same scheme used to quantify the softtissue content, but applied to the barium segment of the data set, we found a Vol. Ratio of about $0.1 \%$ for barium in the AA lung (Table 1). The barium concentration in the two healthy controls $\mathrm{CN} 1$ and $\mathrm{CN} 2$ was below 1\%o and therefore appear as 0 in Table 1 . The Blk mouse that did not receive bariumfilled $\mathrm{M} \Phi(\mathrm{Blk})$ was used to set up the threshold and consequently shows no barium content within the whole lung. The Tukey intervals for barium content (data not shown) are all positive and therefore confirm that the Vol. Ratio of the barium content is significantly larger in the AA lung sample than in the Blk and $\mathrm{CN}$ mice.

In summary, bronchial wall thickening and airway obstruction in asthmatic mouse lung tissue can be visualized in fXPCT data sets [Figs. 3, 4(a) and 4(b)]. Instilled MФ appear within clusters around the bronchial walls [Figs. 3, 4(c) and $4(d)$ ]. With our approach we can also quantify these alterations using automatically generated 3D morphologic parameters. These parameters, i.e. Vol. Ratio of air, barium and lung soft-tissue and St. Th. of air and lung soft-tissue, showed significant differences between AA and healthy controls in accordance with the known pathological features in lungs of an asthma mouse model.

\subsection{Fluorescence microscopy and HR microCT confirm the accumulation of $M \Phi$ in asthmatic lung tissue}

In order to confirm the accumulation and to further analyse the location of the instilled barium-filled and DiD-labelled M $\Phi$ within the lung observed by fXPCT, we performed fluorescence microscopy and HR microCT on lung sections.
Since the preparation process and the analysis at the SYRMEP beamline resulted in the deterioration of the samples and excluded histological analysis, fluorescence microscopy and HR microCT were performed with lungs of a different cohort of mice, but strictly following the same protocol for asthma induction and instillation of $\mathrm{M} \Phi$ (Fig. 1), including the use of the same batch of OVA.

Representative results of the fluorescence microscopy performed on cryosections of a lung of an asthmatic mouse (AA) and a control lung (CN) are shown in Figs. 5(a) and 5(b). Instilled barium- and DiD-labelled $M \Phi$ are shown in red, DAPI stained nuclei appear blue and an anti-CD68 antibody (Ab) which binds to both the applied and endogenous $M \Phi$ is shown in green. As a result, the instilled barium- and DiDlabelled MФ appear yellow in the merged image. We observed DiD-labelled $\mathrm{M} \Phi$ in the small alveoli [Figs. 5(a) and 5(b), white arrows] in both the $\mathrm{CN}$ and the AA lung samples. As already seen in the fXPCT analysis, more instilled M $\Phi$ are present in the AA lung. Moreover, only in the asthmatic tissue were we able to detect an accumulation of instilled $\mathrm{M} \Phi$ within lung areas of high cellular density [Fig. 5(a), detailed view]. These clusters of cells were found throughout the lung and in close proximity to the bronchi. Additionally, a higher number of endogenous $\mathrm{M} \Phi$ (green) was found in the asthmatic lung [Fig. 5(a), green staining].

HR microCT examination clearly verified the presence of the barium-filled $\mathrm{M} \Phi$ inside the lung (dark spots), as shown in Figs. 5(c) and 5(d), for two lung sections from an asthmatic and a control mouse, respectively. In both asthmatic and healthy lungs, barium-filled $\mathrm{M} \Phi$ were detected throughout the lung tissues. However, in contrast to the healthy sample, the asthmatic sample additionally displayed areas around the bronchi with an increased accumulation of M $\Phi$ (white rectangle). Furthermore, thickening of the bronchial walls was observed in the asthmatic tissue [Fig. 5(c), white arrow head] compared with the healthy control [Fig. $5(d)$ ], reflecting the presence of the disease.

Figs. 5(e) and 5(f) show PAS-stained histological slices from lungs of an OVA-induced asthma mouse (AA) and a healthy control $(\mathrm{CN})$. Red dots indicate that mucus production is solely present in the asthmatic lung (Fig. 5e). Furthermore, airway wall thickening can be observed (black arrow head) in the AA slide only. These findings represent typical characteristics of asthma and confirm the presence of an acute asthma reaction in these mice. In addition, ELISA analysis of the sera revealed increased $\mathrm{IgG} 1$ titre for all asthma mice (data not shown), verifying a successful immunization.

\section{Discussion}

This study presents a novel functional in-line free propagation X-ray phase-contrast CT imaging approach (fXPCT) that enables the depiction of both structural features of lung tissue and the accumulation of barium-labelled M $\Phi$ in the lungs of mice after intratracheal instillation. We show that fXPCT can be applied for CT-based immune cell tracking studies. Furthermore, this method allows the quantification of struc- 
tural changes in whole asthmatic lungs in situ by measuring parameters like the volume ratios of air, soft-tissue and the mean airway and soft-tissue thickness, thereby assessing hallmarks of asthma such as bronchial wall thickening and airway obstruction.

By applying a single-distance phaseretrieval algorithm (Paganin et al., 2002) to decouple phase from absorption information, we raised the CNR of lowabsorbing unstained lung soft-tissue by a factor of ten when compared with classical FBP (Mohammadi et al., 2014; Chen et al., 2010). It has to be emphasized that this factor is strongly related to the experimental setup used, including the sample-to-detector distance, the type of samples, the pixel size of the camera system, the quality of the X-ray beam, the phase-retrieval and reconstruction algorithms, and does therefore not represent a general rule for comparing phase-retrieved with non-phase-retrieved CT reconstructions (Donnelly et al., 2003). In a different setup even a factor of up to 200 was reported by Beltran et al. (2011). Another way to improve the detectability of contrast agents would be $K$ edge imaging (Cormode et al., 2010), in spite of the increase in sensitivity in $K$-edge imaging in combination with a non-energy-resolved detector requiring two scans with at least one using a photon energy higher than optimal for soft-tissue contrast.

Single-distance phase retrieval uses, as the name implies, only one projection to calculate the $\delta$-distribution of the refractive index. Therefore, it simplifies the experimental setup and minimizes the acquisition time. This is of particular advantage for unfixed biological samples as used in this study, as it reduces the influence of alterations that occur within the samples over
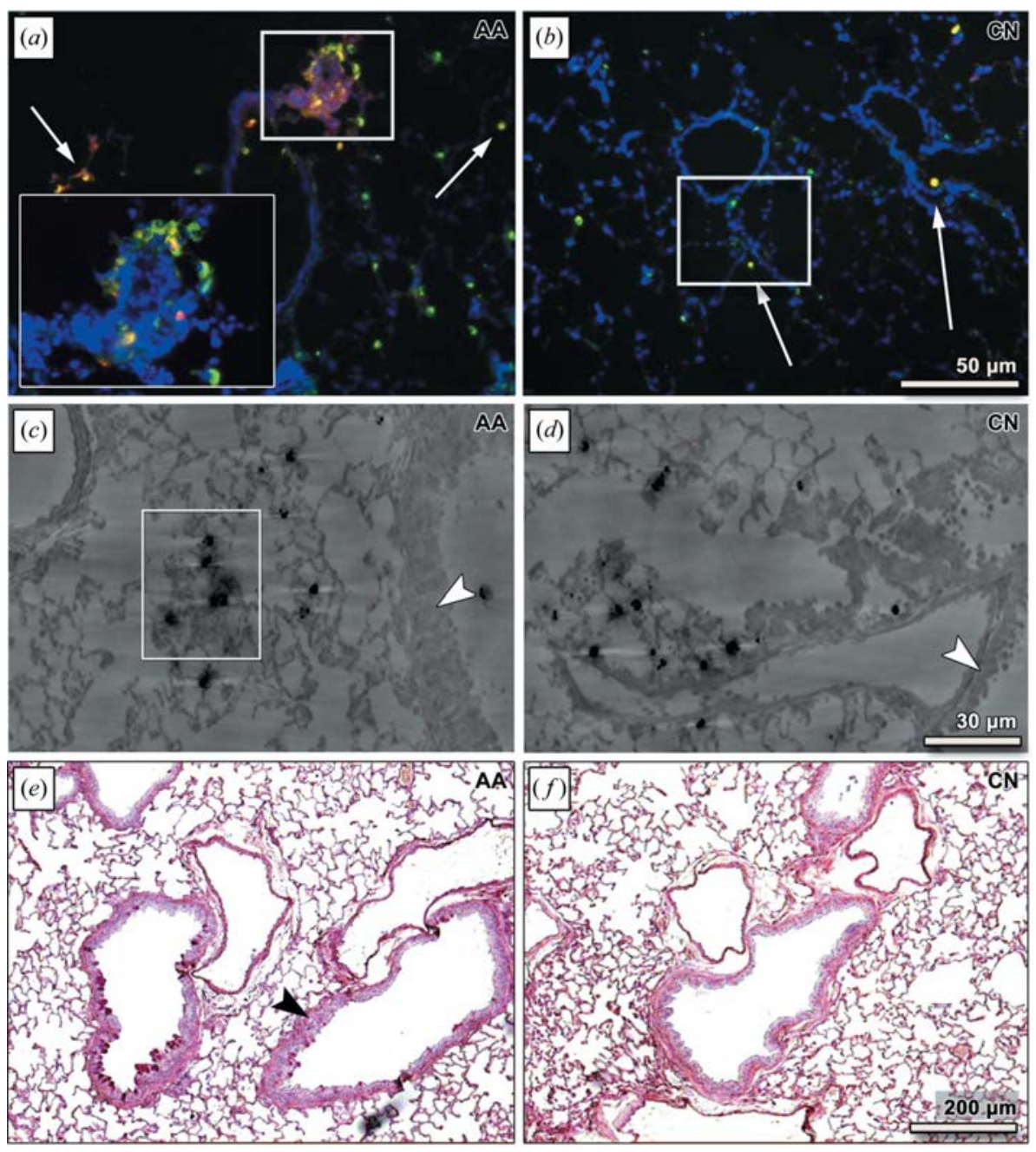

\section{Figure 5}

Validation of the fXPCT results by fluorescence microscopy, HR microCT and histology. $(a, b)$ Fluorescence microscopy of lung sections of $(a)$ an asthmatic (AA) and $(b)$ a control mouse $(\mathrm{CN})$. Nuclei were stained with DAPI (blue) and $M \Phi$ were immuno-stained with an anti-CD68 antibody (green). DiD-labelled $M \Phi$ are shown in red, whereas instilled $M \Phi$ appear yellow due to the double staining with CD68 and DiD. Endogenous M $\Phi$ are shown in green and can be seen in alveoli of both AA and CN lungs, but seem to form clusters in AA ( $a$ and $b$ ). Instilled DiD-labelled M $\Phi$ are also visible in both AA and $\mathrm{CN}$ lung samples (white arrows). In contrast to the $\mathrm{CN}$, the AA sample shows high cellular density areas (white rectangle and detailed view), where several CD68 positive cells and clusters of instilled $\mathrm{M} \Phi$ can be observed. Unlike the AA sample, the $\mathrm{CN}$ does not show these areas, which may explain the increased soft-tissue ratio and the airway obstruction found in the fXPCT results. $(c, d)$ Representative cross sections of $\mathrm{HR}$ microCT for AA and $\mathrm{CN}$ vibratome lung section. An increased wall thickness (white arrow head) for AA $(c)$ and the location of the labelled $\mathrm{M} \Phi$ (black dots) can be clearly depicted. M $\Phi$ can be seen in both $\mathrm{CN}$ and AA, $(c)$ shows an area with higher cell density and accumulation of $M \Phi$ (white rectangle). ( $e$ and $f$ ) PAS-stained lung sections of an asthmatic (AA) and a control mouse (CN). Increased wall thickness in asthmatic lung tissue (black arrow head) is depicted. Red dots are exclusively visible in asthmatic lungs and depict mucus production.

time. However, single-distance phase retrieval is in principle based on the assumption that the studied sample is a 'homogeneous' object, meaning it only contains one known $\delta$-to- $\beta$ ratio (Chen et al., 2011; Gureyev et al., 2009$)$. Since this is not the case for our biological samples, a $\delta$-to- $\beta$ ratio optimized for lung tissue was used. Lung is only composed of low- $Z$ materials $(Z<10)$ for which Wu et al. (2005) showed already that single-distance phase-retrieval algorithms can be applied. Nevertheless, the generated reconstructions are not valid for the analysis of dense struc- tures like bony components (Beltran et al., 2011). If the skeleton is of interest, an additional phase-retrieval step in combination with a different $\delta$-to- $\beta$ ratio should be applied as shown by Beltran et al. (2011). Here we consider a low concentration of barium-sulfate-loaded $\mathrm{M} \Phi$ (less than $0.2 \%$ ) only a minor disturbance of the assumed 'homogeneity' within the lung although barium has a $Z$ value of 56. The calculated ten-fold increase in CNR is based on the comparison with a scan that uses the same setup, but with a 
minimized sample-to-detector distance, generating projection images with predominately absorption-based contrast. Therefore, the reconstructed absorption-based data set was acquired with quasi-monochromatic X-rays known to generate higher-quality data than conventional microCTs with the same spatial resolution (Sera et al., 2005). Thus, the gain in contrast with single-distance phase retrieval is even more prominent when compared with conventional benchtop microCT scans. This increase in contrast directly leads to an increased sensitivity, which in our case did not only enable the depiction but also the quantification of parameters of structural changes in the entire asthmatic lung in 3D, namely the volume ratios of air, soft-tissue and the mean airway thickness, and was the prerequisite for the detection of the labelled $M \Phi$.

In contrast to other studies that analyse mouse lung structure at a micrometre scale (Yong et al., 2009; Sera et al., 2005; Kitchen et al., 2005), we kept the lung in situ by filling the airways with air at a constant pressure. We believe that in this way the shape of the lung, which is critically dependent on the pressure and the boundaries given by the ribcage, can be compared with an in vivo situation. In previous studies using the same asthma mouse model and sample preparation scheme, we found that the overall lung volume (soft-tissue and air content) was greater in asthmatic than in the healthy mice. That means that the asthmatic lungs can be more inflated at the same pressure pointing to a change in the elasticity of the lung tissue. This finding may be reflected in the altered $\delta$-value distribution of the soft-tissue in the asthmatic sample and supports the understanding that in asthma, in addition to inflammation, changes in the composition of lung tissue occur, that lead to a loss in elastic recoil. This loss of elasticity was already observed in patients (Gelb \& Zamel, 2002; Gelb et al., 2002) and confirmed our previous studies.

With this study we present a novel set of parameters [volume ratio (Vol. Ratio) and structural thickness (St. Th.) of airways and soft-tissue] and show their potential use in describing differences between asthmatic and healthy lung tissue. By applying fXPCT we determined a $17 \%$ reduced air content, a $32 \%$ reduced mean airway thickness and a $61 \%$ increase in the soft-tissue content in the asthmatic lung tissue compared with controls, demonstrating that in contrast to histology or conventional CT our approach can precisely measure structural alterations and illustrate them in 3D. The inflammatory response in asthma eventually leads to bronchial obstruction, caused by structural abnormalities such as hypertrophy of airway smooth muscle, sub-epithelial fibrosis, goblet cell hyperplasia, and proliferation of airway blood vessels and nerves. Since routinely most of these morphological changes of airway remodelling are visualized histologically in lung sections in both humans and mouse models (Al Heialy et al., 2011; Blacquière et al., 2010; Leong \& Huston, 2001; Epstein, 2004), the quantification of these changes has been difficult to date. While there are limited approaches by conventional CT and MRI, these techniques are restricted by a low resolution. In particular, minor alterations of the lung tissue in asthma cannot be visualized with the limited spatial resolution of clinical CT scanners. Therefore, up to now the diagnosis of asthma in CT is based on lung densitometry measuring air trapping caused by disturbed ventilation of the lung (Washko et al., 2012). Our approach provides novel parameters which may used in both preclinical asthma models and clinical practice to classify and monitor asthma of different severity and/or to access the influence of an asthma therapy on airway remodelling.

In addition to anatomical information, the increased sensitivity of our fXPCT technique in comparison with conventional microCT allows for the depiction of the distribution of i.t. instilled barium-sulfate-loaded immortalized alveolar macrophages as 'physiological' contrast agent within the 3D lung structure. This, to our knowledge, represents the first approach of using barium-sulfate-loaded macrophages as contrast agents for CT-based cell tracking studies in an experimental allergic airways disease model. So far the use of synchrotron-radiation-based CT for cell tracking has only been reported in a few applications such as imaging small clusters of tumour cells ex vivo loaded with gold nanoparticles (Astolfo et al., 2013). The visualization of pancreatic islet cells encapsulated in barium microcapsules is another example for CT-based cell tracking (Arifin et al., 2012). We found a higher barium content in the asthmatic lung sample, which is most likely due to a more clustered distribution of the instilled macrophages in cell-dense areas around the bronchi, a finding which needs further investigation by high-resolution tomography. This result is supported by our validation experiments with fluorescence microscopy and $\mathrm{HR}$ microCT, all techniques that share a higher spatial resolution than the used fXPCT approach. In our experiment in asthmatic lung tissue, $24 \mathrm{~h}$ after instillation, $M \Phi$ were predominately found in areas with an increased cell density. While the implications of these findings for the pathomechanism of asthma and other lung disease models require further investigation, our results demonstrate the benefit of our imaging technique for 3D localization of cells in specific tissue regions. As the M $\Phi$ used in this study are derived from an immortalized cell line (Mbawuike \& Herscowitz, 1989), no conclusions towards a similar behaviour of endogenous $\mathrm{M} \Phi$ can be made.

A current drawback of our method for future clinical application is the lack of clinical CT systems that provide phase contrast. This limitation may soon be overcome by the implementation of novel technologies such as liquid-metal jet anode systems, miniaturized synchrotrons or grating-interferometer-based phase-contrast imaging utilizing classical X-ray sources (Pfeiffer et al., 2006; Tompkins et al., 1998; van Heekeren et al., 2011). All of these techniques perform well under laboratory conditions but struggle with different technical problems, which limit their use in the clinic so far. In particular, the high radiation dose for grating-based phasecontrast devices hamper their clinical application at the moment (Raupach \& Flohr, 2011). However, our quantification scheme for analysis of lung structure alterations can be directly translated to any other CT or anatomical lung imaging technique, providing a spatial resolution high enough to resolve the lung substructure. 
Our approach presents a proof-of-principle study for fXPCT imaging that specifically visualizes and quantifies morphological differences and airway remodelling of the mouse lung and will support the preclinical validation of newly developed targeted diagnostics and drug delivery strategies for lung diseases. Furthermore, our novel imaging approach provides a solid system for cell tracking studies of immune cells to investigate the role that macrophages might play in the development and progression of lung diseases such as asthma.

This study was conducted within the European project Public Private Partnership for Asthma Imaging and Genomics (P3AGI, http://www.p3agi.eu) funded by the European Commission through FP7-IAPP Marie Curie Action (GA 230739) and was further funded by the Deutsche Forschungsgemeinschaft (DFG, DU 1403/1-1) and the EXTREMA COST action MP1207. Histology and optimization of the macrophage cultivation and labelling approach as well as preliminary microCT analysis was performed at the University Medical Center, Göttingen, Germany, with excellent technical assistance from Sarah Greco, Bärbel Heidrich and Rosi Streich. The beamline experiments were performed at the SYRMEP beamline of the Elettra Synchrotron (Trieste, Italy), all of whose members contributed to the success of these experiments, in particular Dr Diego Dreossi and Dr Nicola Sodini. Cell preparation and labelling, optimization of the sample preparation process and the in vivo animal procedures for the beamline experiments were performed at CBM and in the CBM Animal Facility, Trieste, Italy, situated in near proximity to the synchrotron. This unique situation was vital for this study, to ensure the usage of freshly prepared biological samples. The authors also gratefully acknowledge Dr Tim Gureyev (CSIRO, Australia) for fruitful discussions on the use of the phase-retrieval algorithms. In addition, the authors thank Stephen Kilfeather (Aeirtec; Newcastle, UK) for intense discussions and explanations of the pathomechanism of asthma.

\section{References}

Abràmoff, M. D., Magalhães, P. J. \& Ram, S. J. (2004). Biophoton. Int. 11, 36-42.

Ahrens, E. T. \& Bulte, J. W. M. (2013). Nat. Rev. Immunol. 13, 755763.

Al Heialy, S., Mcgovern, T. K. \& Martin, J. G. (2011). Respirology, 16, 589-597.

Arifin, D. R., Manek, S., Call, E., Arepally, A. \& Bulte, J. W. M. (2012). Biomaterials, 33, 4681-4689.

Astolfo, A., Schültke, E., Menk, R. H., Kirch, R. D., Juurlink, B. H., Hall, C., Harsan, L.-A., Stebel, M., Barbetta, D. \& Tromba, G. (2013). Nanomed. Nanotechnol. Biol. Med. 9, 284-292.

Bang, B. R., Chun, E., Shim, E. J., Lee, H. S., Lee, S. Y., Cho, S. H., Min, K. U., Kim, Y. Y. \& Park, H. W. (2011). Exp. Mol. Med. 43, 275-280.

Beltran, M. A., Paganin, D. M., Siu, K. K. W., Fouras, A., Hooper, S. B., Reser, D. H. \& Kitchen, M. J. (2011). Phys. Med. Biol. 56, 7353-7369.

Beltran, M. A., Paganin, D. M., Uesugi, K. \& Kitchen, M. J. (2010). Opt. Express, 18, 6423.
Biffi, S., Dal Monego, S., Dullin, C., Garrovo, C., Bosnjak, B., Licha, K., Welker, P., Epstein, M. M. \& Alves, F. (2013). PloS One, 8, e57150.

Blacquière, M. J., Hylkema, M. N., Postma, D. S., Geerlings, M., Timens, W. \& Melgert, B. N. (2010). Int. Arch. Allergy Immunol. 153, 173-181.

Bousso, P. \& Moreau, H. D. (2012). Nat. Rev. Immunol. 12, 858864.

Brun, F., Mancini, L., Kasae, P., Favretto, S., Dreossi, D. \& Tromba, G. (2010). Nucl. Instrum. Methods Phys. Res. A, 615, 326-332.

Chen, P. F., Luo, Y. L., Wang, W., Wang, J. X., Lai, W. Y., Hu, S. M., Cheng, K. F. \& Al-Abed, Y. (2010a). Mol. Med. 16, 400-408.

Chen, R.-C., Dreossi, D., Mancini, L., Menk, R., Rigon, L., Xiao, T.-Q. \& Longo, R. (2012). J. Synchrotron Rad. 19, 836-845.

Chen, R. C., Xie, H. L., Rigon, L., Longo, R., Castelli, E. \& Xiao, T. Q. (2011). Opt. Lett. 36, 1719-1721.

Chen, R., Xie, H., Rigon, L., Du, G., Castelli, E. \& Xiao, T. (2010b). Tsinghua Sci. Technol. 15, 102-107.

Cloetens, P., Ludwig, W., Baruchel, J., Van Dyck, D., Van Landuyt, J., Guigay, J. P. \& Schlenker, M. (1999b). Appl. Phys. Lett. 75, 2912.

Cloetens, P., Ludwig, W., Baruchel, J., Van Dyck, D., Van Landuyt, J., Guigay, J. P. \& Schlenker, M. (1999a). Proc. SPIE, 3772, 279-290.

Collins, T. J. (2007). BioTechniques, 43, 25-30.

Cormode, D. P., Naha, P. C. \& Fayad, Z. A. (2014). Contrast Media Mol. Imaging, 9, 37-52.

Cormode, D. P., Roessl, E., Thran, A., Skajaa, T., Gordon, R. E., Schlomka, J.-P., Fuster, V., Fisher, E. A., Mulder, W. J. M., Proksa, R. \& Fayad, Z. A. (2010). Radiology, 256, 774-782.

Cortez-Retamozo, V., Swirski, F. K., Waterman, P., Yuan, H., Figueiredo, J. L., Newton, A. P., Upadhyay, R., Vinegoni, C., Kohler, R., Blois, J., Smith, A., Nahrendorf, M., Josephson, L., Weissleder, R. \& Pittet, M. J. (2008). J. Clin. Invest. 118, 4058-4066.

Donnelly, E. F., Price, R. R. \& Pickens, D. R. (2003). Med. Phys. 30, 2888.

Dreossi, D., Abrami, A., Arfelli, F., Bregant, P., Casarin, K., Chenda, V., Cova, M. A., Longo, R., Menk, R., Quai, E., Quaia, E., Rigon, L., Rokvic, T., Sanabor, D., Tonutti, M., Tromba, G., Vascotto, A., Zanconati, F. \& Castelli, E. (2008). Eur. J. Radiol. 68, S58-S62.

Dunn, O. J. (1961). J. Am. Stat. Assoc. 56, 52-64.

Epstein, M. M. (2004). Int. Arch. Allergy Immunol. 133, 84-100.

Fullmer, H. M. (1960). J. Histochem. Cytochem. 8, 113-121.

Gelb, A. F., Licuanan, J., Shinar, C. M. \& Zamel, N. (2002). Chest, 121, 715-721.

Gelb, A. F. \& Zamel, N. (2002). Curr. Opin. Pulm. Med. 8, 50-53.

Golder, W., Viermetz, M. \& Gmeinwieser, J. (1991). Fortschr. Röntgenstr, 155, 562-567.

Gureyev, T. E., Mayo, S. C., Myers, D. E., Nesterets, Y., Paganin, D., Pogany, A., Stevenson, A. W. \& Wilkins, S. W. (2009). J. Appl. Phys. $\mathbf{1 0 5}, 102005$.

Gureyev, T., Mohammadi, S., Nesterets, Y., Dullin, C. \& Tromba, G. (2013). J. Appl. Phys. 114, 144906.

Heekeren, J. van, Kostenko, A., Hanashima, T., Yamada, H., Stallinga, S., Offerman, S. E. \& van Vliet, L. J. (2011). Med. Phys. 38, 5136 .

Hildebrand, T. \& Ruegsegger, P. (1997). J. Microsc. 185, 67-75.

Hooper, S. B., Kitchen, M. J., Siew, M. L. L., Lewis, R. A., Fouras, A. B., te Pas, A., Siu, K. K. W., Yagi, N., Uesugi, K. \& Wallace, M. J. (2009). Clin. Exp. Pharmacol. Physiol. 36, 117-125.

Hooper, S. B., Kitchen, M. J., Wallace, M. J., Yagi, N., Uesugi, K., Morgan, M. J., Hall, C., Siu, K. K. W., Williams, I. M., Siew, M., Irvine, S. C., Pavlov, K. \& Lewis, R. A. (2007). FASEB J. 21, 33293337.

Keyriläinen, J., Bravin, A., Fernández, M., Tenhunen, M., Virkkunen, P. \& Suortti, P. (2010). Acta Radiol. 51, 866-884.

Kitchen, M. J., Lewis, R. A., Yagi, N., Uesugi, K., Paganin, D., Hooper, S. B., Adams, G., Jureczek, S., Singh, J., Christensen, C. R., Hufton, A. P., Hall, C. J., Cheung, K. C. \& Pavlov, K. M. (2005). Br. J. Radiol. 78, 1018-1027. 
Kitchen, M. J., Paganin, D., Lewis, R. A., Yagi, N., Uesugi, K. \& Mudie, S. T. (2004). Phys. Med. Biol. 49, 4335-4348.

Leong, K. P. \& Huston, D. P. (2001). Ann. Allergy Asthma Immunol. 87, 96-110.

Lewis, R. A., Yagi, N., Kitchen, M. J., Morgan, M. J., Paganin, D., Siu, K. K. W., Pavlov, K., Williams, I., Uesugi, K., Wallace, M. J., Hall, C. J., Whitley, J. \& Hooper, S. B. (2005). Phys. Med. Biol. 50, 50315040.

Markus, M. A., Dullin, C., Mitkovski, M., Prieschl-Grassauer, E., Epstein, M. M. \& Alves, F. (2014). PLoS ONE, 9, e90017.

Mbawuike, I. N. \& Herscowitz, H. B. (1989). J. Leukoc. Biol. 46, 119127.

Mizue, Y., Ghani, S., Leng, L., McDonald, C., Kong, P., Baugh, J., Lane, S. J., Craft, J., Nishihira, J., Donnelly, S. C., Zhu, Z. \& Bucala, R. (2005). Proc. Natl Acad. Sci. 102, 14410-14415.

Mohammadi, S., Larsson, E., Alves, F., Dal Monego, S., Biffi, S., Garrovo, C., Lorenzon, A., Tromba, G. \& Dullin, C. (2014). J. Synchrotron Rad. 21, 784-789.

Moreira, A. P. \& Hogaboam, C. M. (2011). J. Interferon Cytokine Res. 31, 485-491.

Mosmann, T. (1983). J. Immunol. Methods, 65, 55-63.

Nahrendorf, M., Zhang, H., Hembrador, S., Panizzi, P., Sosnovik, D. E., Aikawa, E., Libby, P., Swirski, F. K. \& Weissleder, R. (2008). Circulation, 117, 379-387.

Napp, J., Dullin, C., Müller, F., Uhland, K., Petri, J. B., van de Locht, A., Steinmetzer, T. \& Alves, F. (2010). Int. J. Cancer, 127, 19581974.

Nugent, K. A. (2007). J. Opt. Soc. Am. A, 24, 536-547.

Nugent, K. A. (2010). Adv. Phys. 59, 1-99.

Olendrowitz, C., Bartels, M., Krenkel, M., Beerlink, A., Mokso, R., Sprung, M. \& Salditt, T. (2012). Phys. Med. Biol. 57, 5309-5323.

Paganin, D., Mayo, S. C., Gureyev, T. E., Miller, P. R. \& Wilkins, S. W. (2002). J. Microsc. 206, 33-40,

Pfeiffer, F. Weitkamp, T. Bunk, O. \& David, C. (2006). Nat. Phys. 2 , 258-261.

Raupach, R. \& Flohr, T. G. (2011). Phys. Med. Biol. 56, 22192244.

Ryan, B. F., Joiner, B. L. \& Cryer, J. D. (2005). Minitab Handbook (updated for release 14). New York: Duxbury Press.
Schleede, S., Meinel, F. G., Bech, M., Herzen, J., Achterhold, K., Potdevin, G., Malecki, A., Adam-Neumair, S., Thieme, S. F., Bamberg, F., Nikolaou, K., Bohla, A., Yildirim, A. O., Loewen, R., Gifford, M., Ruth, R., Eickelberg, O., Reiser, M. \& Pfeiffer, F. (2012). Proc. Natl Acad. Sci. 109, 17880-17885.

Sera, T., Uesugi, K., Himeno, R. \& Yagi, N. (2007). Respir. Physiol. Neurobiol. 156, 304-311.

Sera, T., Uesugi, K. \& Yagi, N. (2005). Med. Phys. 32, 2787.

Takeda, T., Momose, A., Itai, Y., Wu, J. \& Hirano, K. (1995). Acad. Radiol. 2, 799-803.

Tanaka, T., Honda, C., Matsuo, S., Noma, K., Oohara, H., Nitta, N., Ota, S., Tsuchiya, K., Sakashita, Y., Yamada, A., Yamasaki, M., Furukawa, A., Takahashi, M. \& Murata, K. (2005). Invest. Radiol. 40, 385-396.

Taylor, L. (1981). IEEE Trans. Antennas Propag. 29, 386-391.

Thakur, M. L. (1977). Int. J. Appl. Radiat. Isot. 28, 183-201.

Tompkins, R. J., Mercer, I. P., Fettweis, M., Barnett, C. J., Klug, D. R., Porter, L. G., Clark, I., Jackson, S., Matousek, P., Parker, A. W. \& Towrie, M. (1998). Rev. Sci. Instrum. 69, 3113.

Trivedi, R. A., Mallawarachi, C., U-King-Im, J. M., Graves, M. J., Horsley, J., Goddard, M. J., Brown, A., Wang, L., Kirkpatrick, P. J., Brown, J. \& Gillard, J. H. (2006). Arterioscler. Thromb. Vasc. Biol. 26, 1601-1606.

Verdelis, K., Lukashova, L., Atti, E., Mayer-Kuckuk, P., Peterson, M. G. E., Tetradis, S., Boskey, A. L. \& van der Meulen, M. C. H. (2011). Bone, 49, 580-587.

Washko, G. R., Parraga, G. \& Coxson, H. O. (2012). Respirology, 17, 432-444.

Weitkamp, T., Raven, C. \& Snigirev, A. A. (1999). Proc. SPIE, 3772, 311-317.

Wu, X., Liu, H. \& Yan, A. (2005). Opt. Lett. 30, 379-381.

Yagi, N., Suzuki, Y., Umetani, K., Kohmura, Y. \& Yamasaki, K. (1999). Med. Phys. 26, 2190.

Yang, M., Kumar, R. K., Hansbro, P. M. \& Foster, P. S. (2012). J. Leukoc. Biol. 91, 557-569.

Yong, H. S., Kang, E. Y., Kim, Y. K., Woo, O. H., Shin, B. K., Oh, C. H., Je, J. H., Han, H. \& Seo, J. S. (2009). Yonsei Med. J. 30, 422 426.

Zhang, L., Li, D. \& Luo, S. (2011). PLoS ONE, 6, e17400. 


\section{SCIENTIFIC REP}
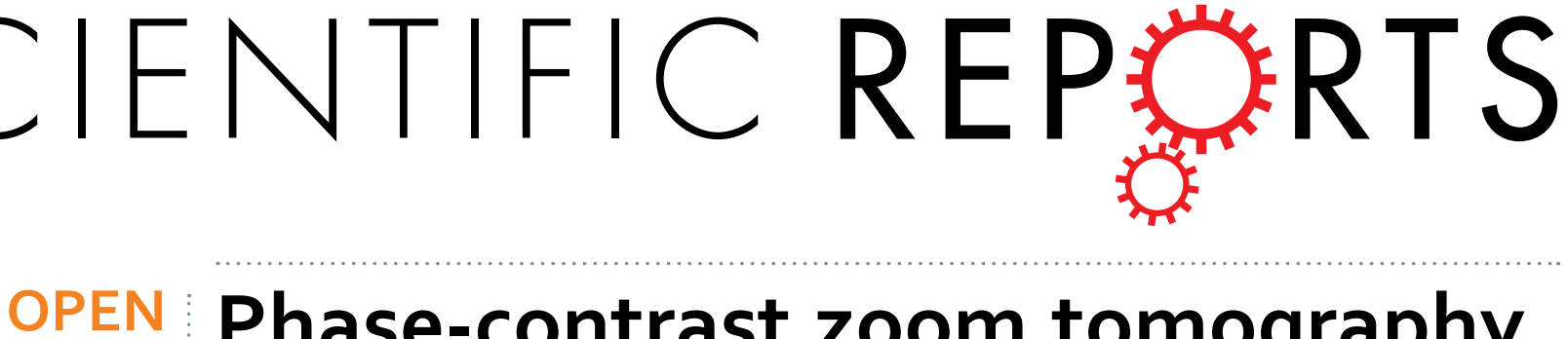

Phase-contrast zoom tomography reveals precise locations of macrophages in mouse lungs

Received: 27 October 2014

Accepted: 24 March 2015

Published: 12 May 2015

\author{
Martin Krenkel ${ }^{1}$, Andrea Markus², Matthias Bartels ${ }^{1}$, Christian Dullin ${ }^{3}$, Frauke Alves ${ }^{2}, 3,4$ \& \\ Tim Salditt ${ }^{1}$
}

We have performed $x$-ray phase-contrast tomography on mouse lung tissue. Using a divergent $x$-ray beam generated by nanoscale focusing, we used zoom tomography to produce three-dimensional reconstructions with selectable magnification, resolution, and field of view. Thus, macroscopic tissue samples extending over several $\mathrm{mm}$ can be studied in sub-cellular-level structural detail. The zoom capability and, in particular, the high dose efficiency are enabled by the near-perfect exit wavefront of an optimized $x$-ray waveguide channel. In combination with suitable phase-retrieval algorithms, challenging radiation-sensitive and low-contrast samples can be reconstructed with minimal artefacts. The dose efficiency of the method is demonstrated by the reconstruction of living macrophages both with and without phagocytized contrast agents. We also used zoom tomography to visualize barium-labelled macrophages in the context of morphological structures in asthmatic and healthy mouse lung tissue one day after intratracheal application. The three-dimensional reconstructions showed that the macrophages predominantly localized to the alveoli, but they were also found in bronchial walls, indicating that these cells might be able to migrate from the lumen of the bronchi through the epithelium.

Lung tissue, with its intricate three-dimensional (3D) system comprising the bronchial tree, alveoli, and blood vessels, is an excellent example of how the 3D structures of tissues enable their physiological function. Conversely, structural alterations are associated with different pathological states. To investigate these relationships quantitatively, the $3 \mathrm{D}$ structure of the tissue must be assessed from the cellular to the organ scale. Furthermore, cell-tracking studies are of high interest for the location of cells in relation to anatomical structures. The conventional approach of sectioning histology followed by optical microscopy or electron microscopy is associated with several major deficits and restrictions. Apart from possible slicing or staining artefacts, it is extremely tedious and time-consuming to record an entire organ or large field of view (FOV), making it almost impossible to cover the complete 3D tissue architecture of many different specimens, even at moderate resolution.

In the use of 3D biomedical imaging to fill this gap, $\mathrm{x}$-rays are the first choice due to the required penetration depth and resolution power. X-ray tomography is a powerful technique for imaging high-density ('hard') structures in tissues and bodies that can otherwise only be visualized in a destructive manner. However, the advantage of transparency for hard x-rays is also a considerable drawback for the examination of most non-absorbing ('soft') tissues, resulting in a lack of contrast for low-density tissue. For micron- and nanometre-scale structures, low absorption coefficients become even more restrictive because measurable absorption levels build up only over longer path lengths. A closer

${ }^{1}$ Institute for X-ray Physics, University of Göttingen, 37077 Göttingen, Germany. ${ }^{2}$ Department of Haematology and Medical Oncology, University Medical Center Göttingen, 37075 Göttingen, Germany. ${ }^{3}$ Department of Diagnostic and Interventional Radiology, University Medical Center Göttingen, 37075 Göttingen, Germany. ${ }^{4}$ Department of Molecular Biology of Neuronal Signals, Max-Planck-Institute of Experimental Medicine, 37077 Göttingen, Germany. Correspondence and requests for materials should be addressed to T.S. (email: tsaldit@gwdg.de) or M.K. (email: mkrenke@gwdg.de) 
examination, however, of the optical constants involved in the $\mathrm{x}$-ray index of refraction $n=1-\delta+\mathrm{i} \beta$ shows that, for the elements and photon energies relevant for the tomography of tissues, the refractive decrement $\delta$ is up to three orders of magnitude larger than the absorption component $\beta$. Even if only relative changes, e.g., between water and protein, are relevant for the imaging, this opens up a huge potential to increase the contrast and resolution for soft tissues ${ }^{1-3}$. This alteration can be achieved if interaction via $\delta$ is exploited, i.e., if the phase shift $k \delta z$ of an $\mathrm{x}$-ray wave with wave number $k=2 \pi / \lambda$ propagating through matter over a distance $z$ can be visualized. See ${ }^{4,5}$ for a review.

To this end, several phase-contrast techniques have been developed in recent decades ${ }^{6,7}$. The most important phase-contrast principles are Zernike phase-contrast in zone plate $\mathrm{X}$-ray microscopes ${ }^{8,9}$, grating interferometry ${ }^{10-12}$, scanning diffraction microscopy ${ }^{13,14}$, and phase-contrast formation based on the free propagation of the $\mathrm{x}$-ray beam behind the sample $\mathrm{e}^{15-17}$. Each technique has its advantages and drawbacks, and each can be applied to a certain range of length scales and in certain types of applications ${ }^{18,19}$. For zone plate-based Zernike phase-contrast, resolutions down to ten nanometres have been achieved in $2 \mathrm{D}^{18}$, but a resolution in this range cannot be achieved in tomography of thick specimens ${ }^{9}$. Furthermore, the low efficiencies of the optical elements behind the sample (zone plate, phase annulus) lead to the need for an increased radiation dose, and the calculation of quantitative phase-contrast values is typically based on idealized assumptions for the optical elements. Grating or analyser-based phase-contrast are the methods of choice for macroscopic field of view, with maximum-resolution values of approximately $4 \mu \mathrm{m}^{20}$. Coherent lensless imaging methods are more dose efficient because no optical element is needed behind the sample for image formation. Scanning transmission X-ray microscopy (STXM) with ptychographic phase reconstruction ${ }^{21}$ and coherent diffractive imaging ${ }^{22,23}$ have both reached a resolution of approximately $10 \mathrm{~nm}$ in $2 \mathrm{D}$ for strongly diffracting test structures and sub-50 $\mathrm{nm}$ resolution for single cells. However, a major drawback of these methods is the limited FOV resulting from the small beam size and the scanning overhead. On the organelle ${ }^{23}$ and single-cell levels ${ }^{24,25}$, high-resolution 3D reconstructions have been achieved, and at even finer scales, the FOV can be enlarged in tomographic ptychography ${ }^{26}$. However, this increased resolution comes at the cost of long measurement times in the order of $10 \mathrm{~h}$, which limits the usefulness of this method for objects in the range of several hundred $\mu \mathrm{m}$.

Propagation-based phase-contrast enables a lensless full-field imaging approach compatible with a wide range of sample sizes, resolution values, photon energies and source characteristics. The imaging scheme is conceptually simple: extending the standard radiographic exposure by a free propagation distance between object and detector and enhanced (spatial) coherence enables a defocused image based on self-interference (in-line hologram) to be recorded, which still bears recognizable similarities with the object. The main difficulty is the phase-retrieval step, which is typically based on $a$ priori information regarding the sample (e.g., a compact support ${ }^{27}$ ) or on idealized assumptions (e.g., weakly or non-absorbing objects ${ }^{28}$ ) often combined with intensity measurements at different Fresnel numbers (e.g., different defocus distances) ${ }^{29}$. In $\mathrm{x}$-ray waveguide-based (cone beam) holographic imaging, we have previously demonstrated resolutions below $30 \mathrm{~nm}^{30,31}$ and fields of view in the range of $100 \mu \mathrm{m}$ as well as tomographic 3D reconstructions with nanometre resolution of single cells ${ }^{32}$. Propagation-based phase-contrast tomography of larger tissue samples has also been reported ${ }^{33-36}$.

For the purposes of imaging tissues from several $\mathrm{mm}$ up to entire small animal organs, at a resolution in the range of $100 \mathrm{~nm}$ to several microns, $\mathrm{x}$-ray propagation imaging is the method of choice. However, $3 \mathrm{D}$ visualization of a whole organ such as the lung with nanoscale resolution would require handling unreasonable amounts of data. A suitable approach must therefore allow for 3D imaging of a large FOV on the millimetre scale with the possibility of zooming in to regions of interest (ROIs), yielding information at the nanometre scale to visualize sub-cellular features. Propagation-based phase-contrast in cone beam geometry enables tomography with an effective zoom function as controlled by the focus to object distance $z_{1}$ and the object to detector distance $z_{2}^{32,34,37,38}$, but this method has thus far not been applied to examine biological hydrated soft tissue.

In this work, we have used propagation-based phase-contrast with zoom tomography to measure the 3D density distribution of hydrated lung tissue samples in a large FOV and at high magnification with sub-cellular resolution. To achieve the enhanced nanoscale resolution and high dose efficiency, we have tuned the characteristics of the incoming wave-front (coherence, curvature, reduced aberrations) by mode filtering based on x-ray waveguides, which act as a secondary quasi point source for object illumination. Using this approach, we have then visualized the distribution of barium-labelled macrophages in healthy and asthmatic lung tissues. Although macrophages are known to be involved in allergic inflammation $^{39}$, the exact role of macrophages in asthma is still not well understood ${ }^{40-43}$. To investigate this role, the distribution of macrophages and their migration properties within the lung are important factors requiring $3 \mathrm{D}$ visualization with high resolution and contrast. Using these unprecedented phase-contrast tomography capabilities, we can detect barium-labelled murine alveolar macrophages of the cell line $\mathrm{MH}-\mathrm{S}^{44}$ in asthmatic and healthy lung tissue one day after intratracheal (i.t.) application. We show that macrophages localize predominately to alveoli and are able to penetrate the epithelial layer between the airway lumen and parenchyma. In addition, we demonstrate the dose efficiency and scalable resolution capability of this approach by providing $2 \mathrm{D}$ reconstructions of single living macrophages, with and without phagocytized contrast agents. 


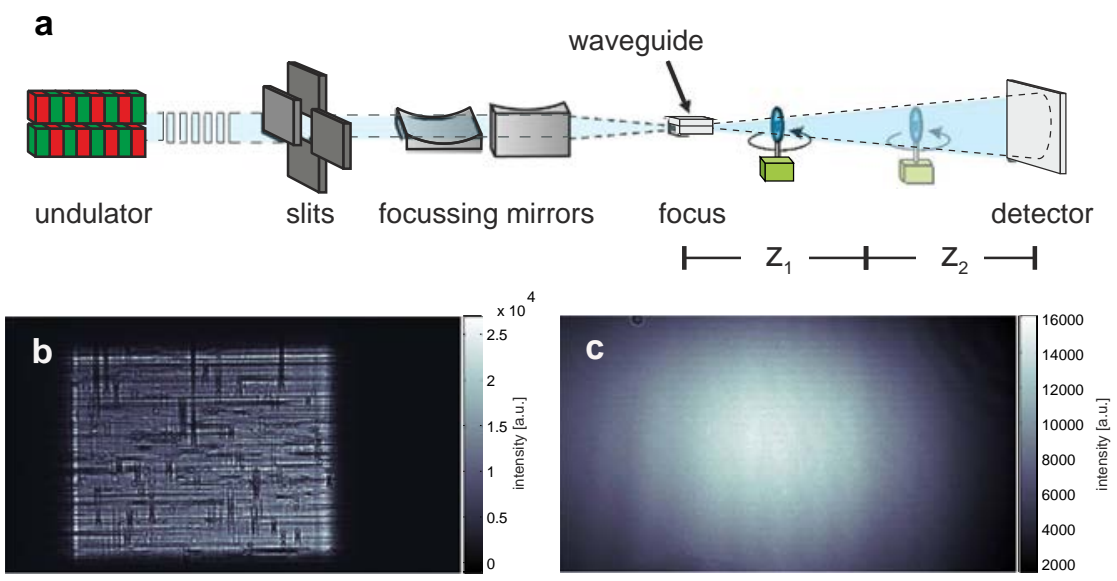

Figure 1. (a) Schematic of the imaging geometry: The $\mathrm{x}$-ray beam is generated in an undulator at a 3rdgeneration synchrotron storage ring. A secondary source is created by focusing the beam with KB mirrors, yielding a divergent beam behind the focus, which makes it easy to control the magnification by changing the defocus distance. The radiation is detected by a scintillation-based detector. (b) A typical KB farfield showing wavefront artefacts due to mirror figure errors. The use of an $\mathrm{x}$-ray waveguide for mode and coherence filtering and for further reduction of the source size down to $20 \mathrm{~nm}$ results in a significantly improved wavefront for the imaging experiment (see (c) for a typical farfield of the waveguide beam).

\section{Experimental Procedure}

Barium sulphate-labelled murine alveolar macrophages of the cell line $\mathrm{MH}-\mathrm{S}^{44}$ were instilled i.t. in asthmatic and control mice $24 \mathrm{~h}$ prior to euthanasia. Cell labelling was achieved by adding a suspension of barium sulphate $\left(\mathrm{BaSO}_{4}\right)$ directly to the media followed by co-incubation. Lung tissue slices were prepared and mounted in phosphate-buffered saline (PBS) between two thin foils immediately before the tomographic measurements. Two different synchrotron setups with divergent beams obtained with Kirkpatrick-Baez (KB) or waveguide (WG) illumination were used for the experiments, resulting in different illuminating beams. The setup is sketched in Fig. 1(a) with resulting farfield intensity distributions shown in Fig. 1(b) for the KB and in (c) for the WG setup, both obtained for comparison at the P10 beamline.

\section{Results}

Holographic imaging of asthmatic mouse lungs. Figure 2 shows three typical empty beam-corrected images of lung tissue samples with labelled macrophages obtained at the ID22 (Fig. 2(c)) and at the P10 beamline, showing a projection for a large FOV (Fig. 2(d)) and the same projection angle at a larger magnification (Fig. 2(e)). Depending on the Fresnel number $F$, different imaging regimes are covered, ranging from the direct contrast regime $(F=1.97)$ in Fig. 2(c) to a strong holographic regime $(F=0.075)$ in Fig. 2(e). The measured intensity in the direct contrast regime is explained by the 2D derivative (Laplacian) of the phase distribution behind the sample. Although nearfield phase-retrieval algorithms based on, e.g., the transport of intensity equation (TIE) ${ }^{45}$ would still be feasible in this imaging regime, we used a more general method based on the contrast transfer function (CTF), which has been described previously ${ }^{46}$. The TIE approximation of small propagation distances fails for larger image frequencies, and the CTF approach delivers results with higher resolution. As an example, 2D phase reconstructions are shown in Fig. 2(f) and (g) and Supplementary Figs. 1 to 3 with corresponding parameters shown in Supplementary Table 1. Phase retrieval was performed individually for each projection of a tomographic scan, and subsequent tomographic reconstruction yielded a 3D dataset.

Figure 3 depicts the results of a reconstructed $3 \mathrm{D}$ volume of a lung tissue slice, recorded with $\mathrm{KB}$ illumination in the direct contrast regime at ID22. The rendering in Fig. 3(a) shows three orthoslices: a semi-automatically segmented blood vessel (purple), automatically labelled soft tissue (half transparent yellow) in a ROI in the centre of the volume, and automatically labelled barium sulphate (green). Several bronchial tubes can be seen with a typical wall morphology showing single goblet cells protruding into the airways. A single planar orthoslice is shown in Fig. 3(b). The complete information contained in the datasets can be assessed from a video of the 3D rendering provided as Supplementary Video 1. With the high flux available at the ID22 beamline, a tomographic scan takes only approximately 15 minutes. A total of nine successful tomographic scans were recorded from five different mice: two asthmatic mice, two control mice and one blank specimen without instilled macrophages (see Supplementary Fig. 5). By visual inspection, the bronchial walls were found to be slightly thicker in asthmatic compared to control mice, but the location and distribution of macrophages did not seem to differ. In the reconstructed 

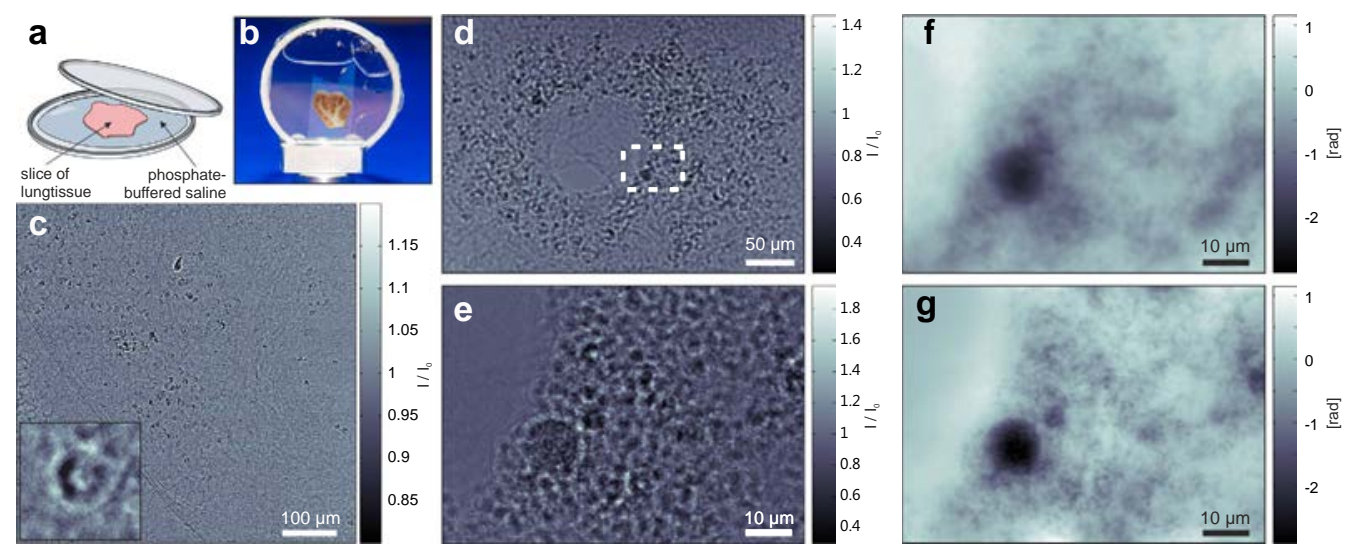

Figure 2. Sample preparation and measurements: (a) Sketch of sample mounting. The $500 \mu \mathrm{m}$ thick mouse lung tissue slices were placed in buffer solution on a thin foil and covered with a second foil. (b) Photograph of a final prepared sample mounted on a sample holder. (c,d,e) Phase-contrast projections of lung tissue samples corresponding to single projections out of the tomographic scans shown in Fig. 3 to 5: (c) A typical empty beam-corrected phase-contrast image recorded at the ID22 beamline showing direct contrast of the lung structure. The inset shows a detailed view scaled to have the same pixel size as (e). (d) A typical empty beam-corrected phase-contrast image recorded at the P10 beamline. Due to the smaller Fresnel numbers, the image is of holographic nature, showing less correspondence to the real spatial structure. (e) Phase-contrast image at higher geometric magnification (smaller defocus distance $z_{1}$ ) of the area marked by a dashed rectangle in (d). (f) Reconstructed phase distribution out of (d) showing the region inside the dashed rectangle. (g) Reconstructed phase out of (e) and three other distances showing the higher resolution of the zoomed dataset.

volume, the macrophages can be readily rendered based on automatic segmentation of the barium inside the cells. The $2 \mathrm{D}$ orthoslices show that barium- labelled macrophages are found predominantly within alveoli (see Fig. 3(b)). However, only a full 3D analysis can unambiguously distinguish between an adhering and an embedded location. Thus, we have rendered the epithelium together with the macrophages, based on automatic segmentation in a small ROI in the centre of the reconstruction volume, as shown in Fig. 3(a), as well as from a different viewing angle in (c). The positions of the labelled macrophages suggest that many macrophages are surrounded by soft tissue. To investigate this location in further detail, it is necessary to increase the resolution. Note that the present dataset was recorded at a voxel size of $430 \mathrm{~nm}$ and is rendered at $860 \mathrm{~nm}(2 \times$ binning $)$ in Fig. 3. To obtain high-resolution reconstructions, we used a setup based on waveguide optics, as described below.

Zoom tomography using the waveguide setup. Figure 4 shows the results obtained with the waveguide setup at the P10 beamline with the same colour coding as in Fig. 3. Barium within the instilled macrophages was automatically labelled and is rendered in green. A semi-automatically segmented blood vessel is shown in purple. A bronchial tube (not rendered) that branches into two tubes lies parallel to the blood vessel. To provide a detailed impression of the $3 \mathrm{D}$ volume, a video of the rendering is available as Supplementary Video 2. A small part of the bronchial wall, marked by the dashed line in the inset of Fig. 4(b), was manually segmented and is rendered in half-transparent yellow. The outline of a single barium- labelled macrophage inside this ROI was manually segmented, and it is shown in blue (Fig. 4(c)). By moving the sample closer to the effective source, from $z_{1}=190 \mathrm{~mm}$ to $z_{1}=40 \mathrm{~mm}$, we increased the magnification while keeping the central ROI in the reconstruction volume. A second tomographic scan at this zoom setting was performed using the CTF-based reconstruction algorithm based on a four-distance dataset. For each projection, phase retrieval was performed using the resampled and aligned images, followed by tomographic reconstruction, as described in detail in Supplementary Methods 1 and 2. A rendering of the resulting $3 \mathrm{D}$ volume is shown in Fig. 5(c), in which the same bronchial wall segmentation and cell outline as in Fig. 4(c) are shown together with automatically labelled barium in green. A detailed video of the rendering is available as Supplementary Video 3. The macrophages are clearly located inside the bronchial wall, indicating that macrophages are able to migrate through the bronchial epithelium.

Dose and resolution. The higher resolution of the zoomed tomograph in Fig. 5 immediately becomes apparent upon inspection of the barium inside the macrophages. Rather than a homogeneous density, we find individual barium clusters scattered within the cell. The macrophages can be identified by segmenting the envelope around the barium (see the cell rendered in blue), which is exemplified in the dashed 


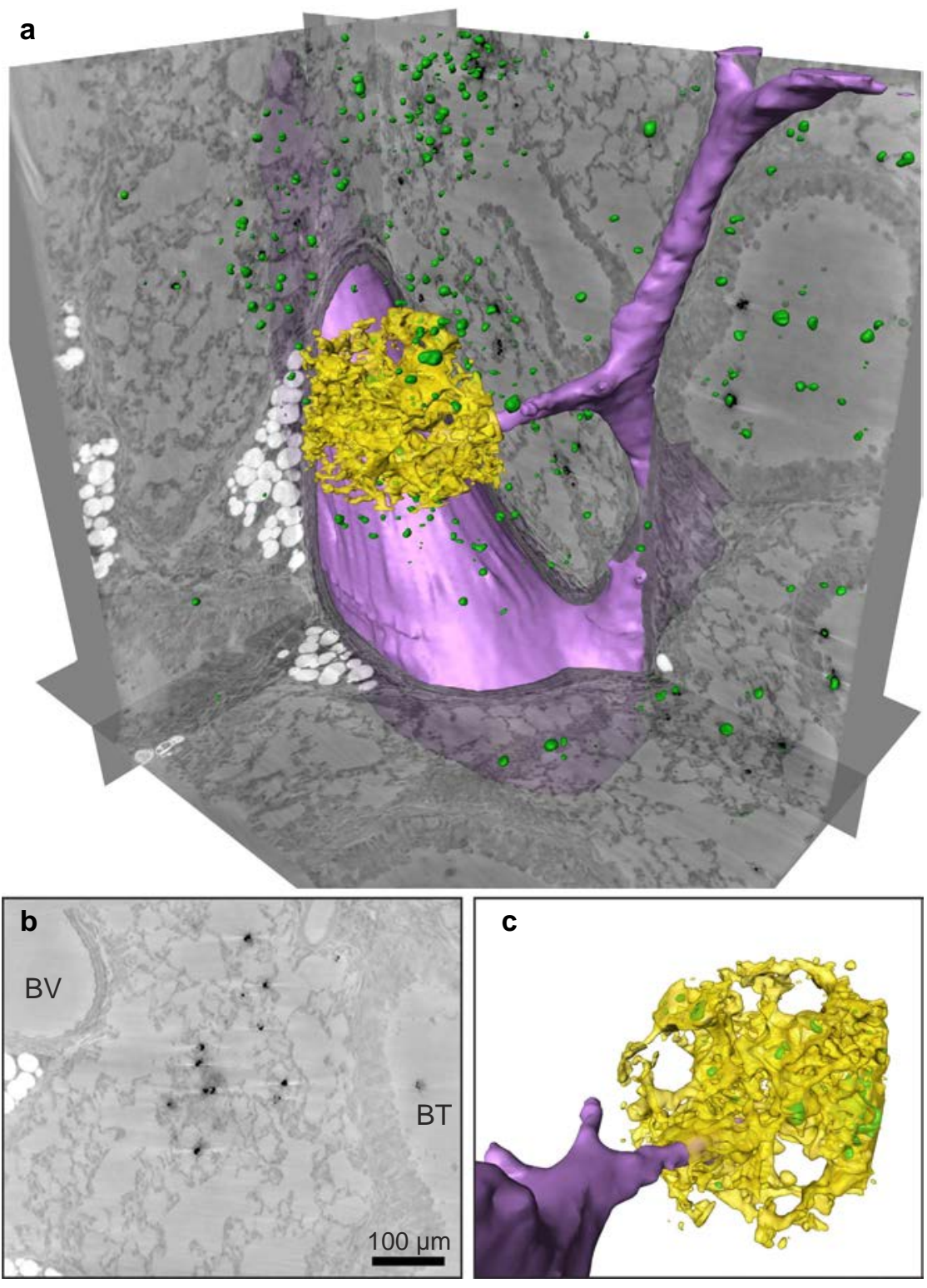

Figure 3. Results obtained in the KB setup: (a) 3D rendering of a reconstructed asthmatic lung tissue volume showing three orthoslices together with automatically labelled (density based) barium clusters (green) and alveolar walls in a small ROI (yellow). A part of a blood vessel was marked semi-automatically (purple). (b) Orthoslice perpendicular to the tomographic rotation axis through the reconstructed volume, as obtained from a single distance tomogram with voxel size $p=430 \mathrm{~nm}$. Darker values correspond to denser material. Barium sulphate particles (black) and fat (white) show a strong density contrast compared to soft tissue. A blood vessel (BV) and a bronchial tube (BT) can be identified based on their different wall morphologies. (c) Close-up of the ROI from a different viewing angle.

red rectangle in (c), as well as in isolated form in (d). The orthoslices in Fig. 5(a,b) correspond to a cut through the same macrophage. A Gaussian fit through a single small particle in this orthoslice reveals a feature size of $249 \mathrm{~nm}$ (see the inset in Fig. 5(b)). Fourier shell correlation indicates a resolution in the $3 \mathrm{D}$ dataset of approximately $170 \mathrm{~nm}$ using a half-bit criterion according to ${ }^{47}$ (see Supplementary Methods 3 for details). Thus, the resolution was increased compared to the results obtained in the KB setup at ID22, but it was significantly worse than the sub-30 $\mathrm{nm}$ resolution achieved using the same waveguide setup with two-dimensional test patterns ${ }^{30}$. This resolution loss can be attributed to small drifts that occur during the time of the tomographic scan, as the dose values (see Table 1) are comparable to those $\mathrm{in}^{30}$. We expect that the resolution might be increased by improving the mechanical stability or by using optimized alignment algorithms. 

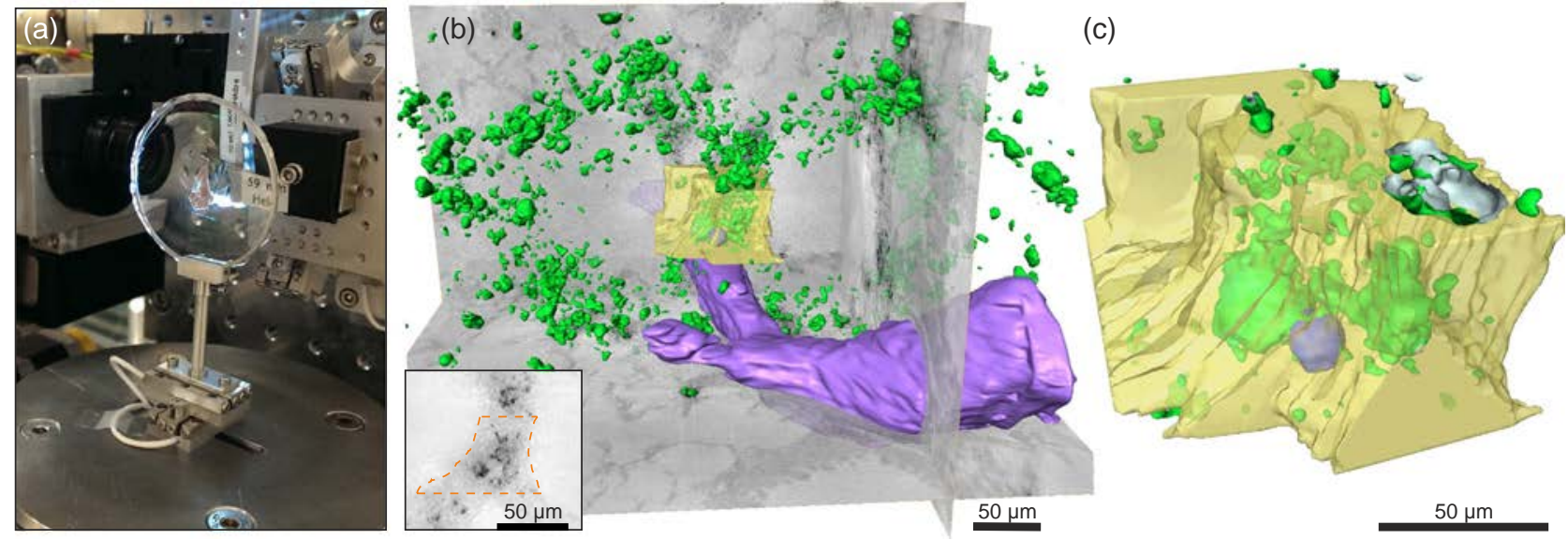

Figure 4. Large FOV results obtained with the waveguide setup: (a) photograph of the sample mounted to the sample holder. (b) 3D rendering of the reconstructed volume of a control lung showing 3 orthoslices together with automatically labelled $\mathrm{BaSO}_{4}$ particles (green), a semi-automatically rendered blood vessel (purple), a manually labelled bronchial wall inside a ROI (yellow), and the contours of a single macrophage in this ROI (blue). An orthoslice through the area used to segment the bronchial wall (dashed orange line) is shown in the inset. (c) Close-up the segmented ROI viewed from a slightly different angle. The same area is measured with a larger magnification, shown in Fig. 5.

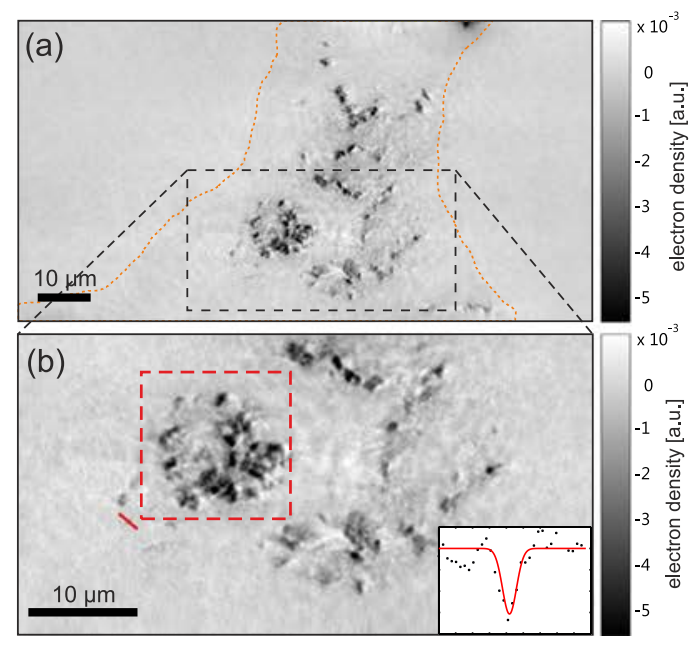

(c)

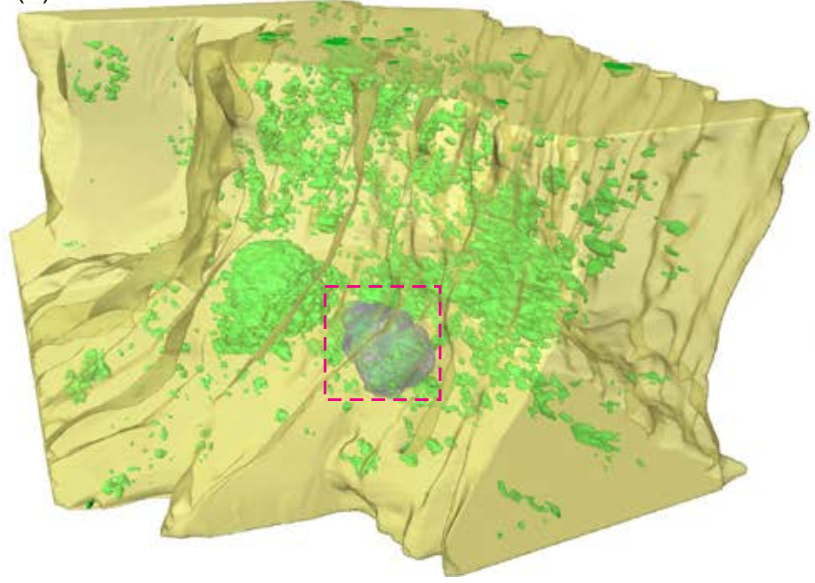

(d)
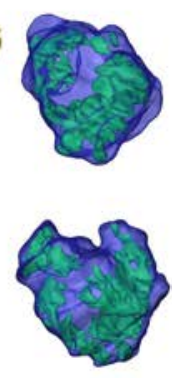

Figure 5. Zoomed-tomography results obtained with the waveguide setup: The sample shown in Fig. 4 was moved closer to the effective source, resulting in a higher geometric magnification. (a) Slice through the 3D volume showing the area used for segmentation of the bronchial wall (dashed orange line). (b) Close-up of the area of the dashed rectangle shown in (a). A profile through the solid line is plotted in the inset showing a feature size of $249 \mathrm{~nm}$ FWHM. The area marked by the red dashed rectangle shows the cell, which is rendered in blue. (c) $3 \mathrm{D}$ rendering of the data, showing automatically labelled aggregates of $\mathrm{BaSO}_{4}$ particles (green), the manually labelled bronchial wall (yellow), and a manually labelled cell outline (blue). (d) Close-up of the barium-labelled cell marked by pink dashed lines in (b) and (c) from two different viewing angles showing the internal barium distribution.

Fluence and dose values for the datasets shown are listed in Table 1. Zoomed tomography at the ID22 beamline was impeded by radiation damage resulting from the higher dose at short defocus distance. Comparing the WG (Fig. 4) with the KB setup (Fig. 3), the dose applied to the sample was 18 times lower, although it was measured at a slightly higher resolution. Note that in ptychographic coherent diffraction imaging, a dose of $2 \cdot 10^{6}$ Gy was required for 3D reconstruction at a similar resolution level ${ }^{14}$. Thus, the dose was 20 times higher than in the zoomed WG dataset shown here. 


\begin{tabular}{|l|c|c|c|c|c|}
\hline & $\begin{array}{c}\text { Fig. } \\
\text { 2(c)/Fig. 3 }\end{array}$ & $\begin{array}{c}\text { Fig. } \\
\text { 2(d,f)/Fig. 4 }\end{array}$ & $\begin{array}{c}\text { Fig. } \\
\text { 2(e,g)/Fig. 5 }\end{array}$ & Fig. 6(a,b) & Fig. 6(c,d) \\
\hline illumination / beamline & KB / ID22 & WG / P10 & WG / P10 & WG / P10 & WG / P10 \\
\hline number of distances & 1 & 1 & 4 & 3 & 5 \\
\hline number of projections & 1500 & 720 & 900 & - & - \\
\hline total exposure time & $150 \mathrm{~s}$ & $2160 \mathrm{~s}$ & $3600 \mathrm{~s}$ & $30 \mathrm{~s}$ & $5 \mathrm{~s}$ \\
\hline distance $z_{1}[\mathrm{~mm}]$ & 299 & 190 & 40 & 39 & 39 \\
\hline voxel size $[\mathrm{nm}]$ & 430 & 245 & 52 & 51 & 51 \\
\hline total fluence $\left[\mathrm{photons} / \mu \mathrm{m}^{2}\right]$ & $1.9 \cdot 10^{8}$ & $6.9 \cdot 10^{6}$ & $2.1 \cdot 10^{8}$ & $1.5 \cdot 10^{6}$ & $2.5 \cdot 10^{5}$ \\
\hline total dose $[\mathrm{Gy}]$ & $5.6 \cdot 10^{4}$ & $3.0 \cdot 10^{3}$ & $9.1 \cdot 10^{4}$ & 633 & 106 \\
\hline
\end{tabular}

Table 1. Experimental parameters and calculated dose. The distance $z_{1}$ indicates the first distance to which all images are resized in case of multiple distances. As Fig. 2 shows individual projections taken out of the full tomographic scan, the total exposure times must be divided by the number of projections.
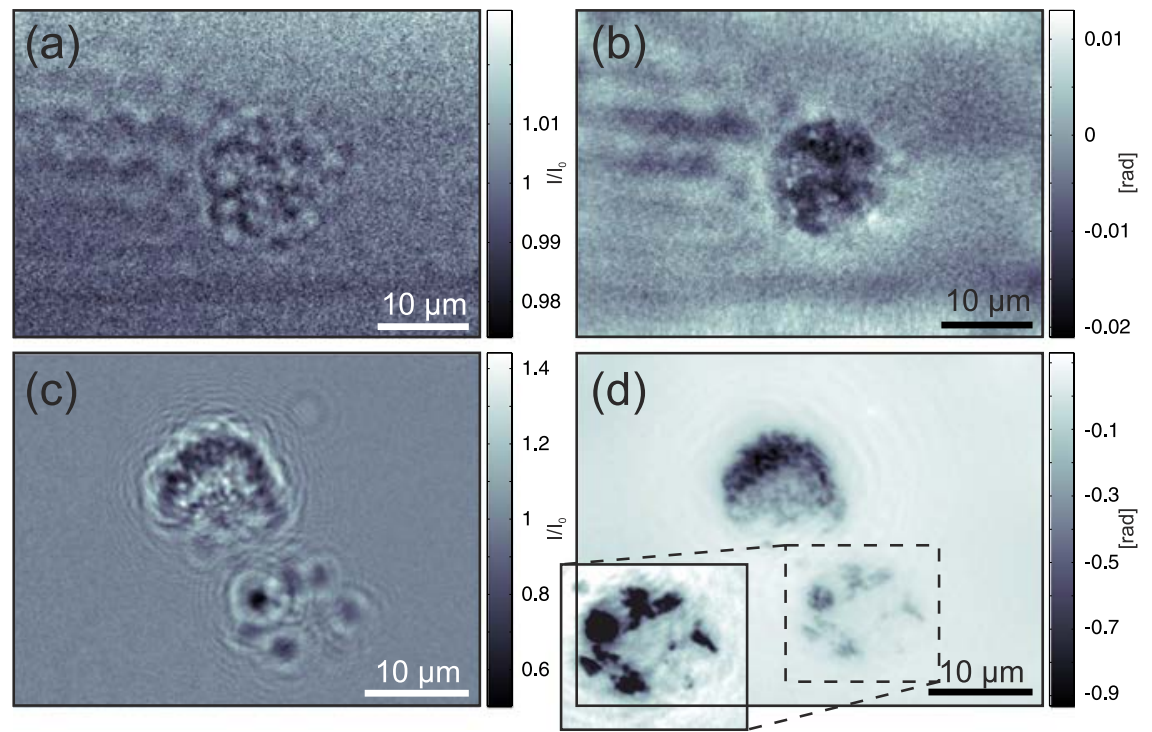

Figure 6. Living single-cell imaging at P10: Living macrophages were kept in medium in an ibidi flow chamber that was mounted onto a sample holder. The left column shows empty beam-corrected phasecontrast images of (a) an unlabelled macrophage and (c) two barium-labelled cells. The right column shows (b) the corresponding phase reconstruction of (a) using 3 distances, each $10 \times 1 \mathrm{~s}$, and (d) the phase reconstruction of (c) using 5 distances, each $1 \mathrm{~s}$. The inset shows the lower right cell with contrast adjusted to enhance the weak signal of the cell structure.

Imaging of living single cells. Motivated by the dose-efficient imaging results for lung tissue, we sought to demonstrate the ability of the method to visualize living macrophages. Apart from being a proof of concept, it was of interest to study in more detail how the barium-based contrast agent is distributed within the cell.

To this end, single macrophages were kept in microfluidic chambers (ibidi microscopy slide) and transported in a mobile incubator to the beamline. The chambers were mounted at $z_{1}=40 \mathrm{~mm}$ behind the effective source, with macrophages adhering to the windows, which are transparent for both $13.8 \mathrm{keV}$ $\mathrm{x}$-rays and visible light, facilitating alignment in the beam. Figure 6 shows holographic phase-contrast images and their corresponding phase reconstructions for a native unlabelled cell $(a, b)$ and for cells that were labelled with the contrast agent by co-incubation with a barium sulphate suspension (c,d). This result proves that single cells can be resolved without contrast agents and shows that the strong contrast in the 3D reconstructions shown in Fig. 3 to Fig. 5 results mainly from the contrast agent. The inset in Fig. 6(d) shows that at low barium concentrations, the cell structure can be observed, although the much stronger contrast of the barium affects the image impression. We also observed that barium sulphate particles are not homogeneously distributed in the cell but, rather form aggregates mainly at the 
periphery. This finding is in agreement with the 3D reconstructions. Depending on the resolution of the image, this clustering of barium sulphate particles can complicate the interpretation of the $3 \mathrm{D}$ data.

\section{Summary and Discussion}

In summary, we have demonstrated that holographic phase-contrast tomography can (i) reveal the 3D structure of hydrated mouse lung tissue slices down to the sub-cellular level and (ii) visualize barium-labelled macrophages within anatomical structures. Using a geometric zoom, imaging at a large field of view in the range of several $100 \mu \mathrm{m}$ and at high resolution down to $170 \mathrm{~nm}$ for selected ROIs was achieved for the same tissue sample without deterioration of its structure. To exploit this zoom capability for soft biological tissues, high dose efficiency is required. We have achieved this efficiency by using $\mathrm{x}$-ray waveguide optics that better fulfil the idealized assumptions on the illuminating wave-field regarding coherence, point-source geometry, and the nearly aberration-free wavefront.

Compared to ptychography, we have obtained reconstructions with a 20 times lower dose at similar resolution. We expect that with sophisticated alignment algorithms and/or faster accumulations, the resolution could be increased even further without increasing the dose. At the same time, the large overhead in data accumulation by scanning microscopy techniques (such as ptychography) are avoided in the present full-field imaging scheme. Naturally, this approach can still be combined with moderate scanning to further enlarge the FOV, which should make it possible to visualize the entire lung of a mouse in 3D. Compared to grating-based phase-contrast imaging, which can also easily cover large FOVs, propagation imaging offers significantly higher resolution down to the sub-cellular level and does not require any optical elements between the sample and the detector.

Persistent challenges in the phase-retrieval step have kept the advantages of propagation imaging from being fully exploited. In particular, in the holographic regime, conventional phase retrieval based on the TIE equation is no longer valid. As detailed in Supplementary Methods 1, we found that the approach based on the contrast transfer function (CTF) pioneered by Cloetens ${ }^{15}$ provides a rapid and robust means to achieve high-quality reconstructions (regarding contrast and resolution) even for low-contrast tissues if the following conditions are met: (i) data are recorded in the holographic regime to optimize contrast transfer for a broad range of spatial frequencies, (ii) the wavefront is sufficiently coherent and well controlled, and (iii) the image series with frames recorded at different magnifications and in different fields of view is well aligned. Further improvements, and in particular contrast values yielding quantitative electron density, can be achieved by extending the phase retrieval to iterative reconstruction algorithms.

Armed with the above imaging methodology, important biomedical questions can now be addressed, which are difficult to address with conventional histology. In particular, phase-contrast zoom tomography could become a novel tool for tracking cells in relation to anatomical structures. The present study on lung tissues in asthmatic and control mice has addressed the distribution of instilled barium-labelled macrophages within the lung. The 3D reconstructions obtained in this study show that one day after i.t. application of MH-S cells, these macrophages were located within the alveolar lumen and within bronchial walls. The low dose required also enabled the imaging of living single cells. Aggregated particles of precipitated barium sulphate were observed in the cytosol of the macrophages. Future experiments will include more sophisticated contrast agents that bind specifically to functional sites inside the macrophages at the organelle level.

\section{Methods}

Sample preparation. The immortalized mouse alveolar macrophage cell line MH-S (American Type Culture Collection, ATCC, USA) was maintained in RPMI medium supplemented with $10 \%$ FCS, $0.05 \mathrm{mM} 2$-mercaptoethanol and $2.06 \mathrm{mM}$ glutamine ${ }^{44}$ in a humidified atmosphere at $5 \% \mathrm{CO}_{2}$ and $37^{\circ} \mathrm{C}$. The cells $\left(1 \times 10^{6} \mathrm{cells} / \mathrm{ml}\right)$ were loaded with barium sulphate particles by co-incubating the cells for $4 \mathrm{~h}$ in standard media containing $3.5 \mu \mathrm{l} / \mathrm{ml}$ of the clinical contrast agent Micropaque CT $\left(7.5 \mathrm{~g} \mathrm{BaSO}_{4} / 150 \mathrm{ml}\right)$ (Guerbet, France). The uptake of $\mathrm{BaSO}_{4}$ differed randomly among cells, but the amount used ensured that most cells would take up enough particles for robust cell tracking. A conventional OVA-induced asthma model was used as previously described ${ }^{48}$. All experiments were carried out in strict accordance with the guidelines for the care and use of laboratory animals of the local ethics office of the University Medical Center Göttingen. This study was approved by the Committee on the Ethics of Animal Experiments of the Niedersächsisches Landesamt für Verbraucherschutz und Lebensmittelsicherheit (LAVES) (Permit Number: 33.12-42502-04-12/0834). All painful procedures were performed under anaesthesia, and all efforts were made to minimize suffering. A total of $1 \times 10^{6}$ barium-labelled macrophages in $30 \mu \mathrm{l}$ PBS were i.t. instilled into the lungs of asthmatic and control BALB/c mice $72 \mathrm{~h}$ after the last OVA challenge under Ketamine 10\% / Xylazine 2\% anaesthesia. Mice were euthanised $24 \mathrm{~h}$ after macrophage instillation using an isoflurane overdose. Lungs were dissected and fixed in $4 \%$ paraformaldehyde for $24 \mathrm{~h}$, and individual lung lobes were embedded in $5 \%$ agarose. Slices $500 \mu \mathrm{m}$ in width were cut using a Leica VT1000 S vibrating blade microtome (Leica, Wetzlar, Germany) and they were stored in $0.02 \%$ sodium azide/PBS solution at $4^{\circ} \mathrm{C}$. Shortly before the measurements at the beamline, the slices were placed between two thin polypropylene foils mounted on $500 \mu \mathrm{m}$ thick aluminium rings; see Fig. 2 . To prevent drying during measurement, the sample holder was sealed. The two rings formed a closed chamber $500 \mu \mathrm{m}$ thick. The samples were mounted vertically on sample holders, which fit the 
corresponding instrument. Photographs of the final mounted samples can be seen in Fig. 2(b) and Fig. 4(a). For imaging of living cells, microscopy slides (ibidi) were used as closed chambers to transport barium-labelled and unlabelled $\mathrm{MH}-\mathrm{S}$ cells in $\mathrm{CO}_{2}$ independent medium to the beamline, keeping the cells at $37^{\circ} \mathrm{C}$ for approximately 4 to $6 \mathrm{~h}$ in a mobile incubator.

KB-based imaging at ID22. The first part of the experiments was carried out at the ID22NI beamline at the European Synchrotron Radiation Facility (ESRF) in Grenoble, France ${ }^{49}$. The x-rays were generated in an undulator and not further monochromatized (pink beam), leading to a very high flux in the order of $10^{12}$ photons per second. The photon energy was set to $17.5 \mathrm{keV}$, with a relative bandwidth $\Delta E / E \approx 0.015$. The beam was focused by two elliptically bent Kirkpatrick-Baez (KB) mirrors to a spot size of less than $100 \mathrm{~nm}$ in both directions, resulting in a divergent beam (8.1 vertical and 8.2 horizontal mrad divergence) that allows tunable magnification by changing the defocus distance $z_{1}$ (see Table 1 ). The effective source size theoretically determines the maximal resolution possible with this setup. No additional optical element was placed in or behind the focus. A fully motorized sample tower positioned the air-bearing rotation stage. A scintillation-based detector was placed at $z_{1}+z_{2}=0.526 \mathrm{~m}$ behind the focal plane. The detector was a high-resolution $\mathrm{x}$-ray microscope based on a thin single-crystal scintillator viewed with a 10 -fold objective lens. The images were captured with a fast read-out low-noise CCD camera (Frelon, $\mathrm{ESRF})^{50}$ resulting in a detector pixel size of $p=0.756 \mu \mathrm{m}$. The propagation can be fully described by a single parameter, the Fresnel-number $F=a /\left(\lambda z_{\text {eff }}\right)$, with the wavelength $\lambda$, the effective propagation distance $z_{\text {eff }}=z_{1} z_{2} /\left(z_{1}+z_{2}\right)$ and a typical feature size $a$. In the present work, we defined a typical feature size of ten pixels $a=10 \cdot p$.

Waveguide-based imaging at the $\mathbf{P}_{\mathbf{1 0}}$. To further increase the resolution, the second part of the experiment was carried out at the GINIX instrument installed at the P10 beamline at DESY in Hamburg, Germany. The x-rays were generated in an undulator, and the energy was set to $13.8 \mathrm{keV}$. The $\mathrm{x}$-rays were monochromatized by a $\mathrm{Si}(111)$ double-slit monochromator, resulting in a relative bandwidth $\Delta E / E=10^{-4}$. The $\mathrm{x}$-rays were focused by $\mathrm{KB}$ mirrors to approximately $100(v) \times 300(h) \mathrm{nm}^{2}$. For high-resolution imaging, the effective source size was further reduced by precisely aligning an $\mathrm{x}$-ray waveguide (WG) system consisting of crossed planar waveguides with an optimized two-component $(\mathrm{Mo} / \mathrm{Ge})$ cladding $^{51}$ in the $\mathrm{KB}$ focus. By multi-modal interference, the waveguide beam propagated through the $59 \mathrm{~nm}$ thick carbon guiding layer over a total length of $0.76 \mathrm{~mm}$, resulting in a reduced beam size in the exit plane of $16 \mathrm{~nm}$ FWHM, as determined by iterative inversion of the measured farfield data $^{31,52}$, confirming the finite difference simulations carried out for optical design. The sample was positioned in the defocus of the beam at a distance $z_{1}$ behind the waveguide exit on a fully motorized sample tower, including an air-bearing rotation. A high-resolution SCMOS x-ray camera (Photonic Science) was used in which a $15 \mu \mathrm{m}$ thick Gadox scintillation screen was directly coupled via a 1:1 fibre-optic plate to the sCMOS chip, resulting in a detector pixel size of $6.54 \mu \mathrm{m}$. The detector was placed at $z_{1}+z_{2}=5.07 \mathrm{~m}$ behind the effective source created by the WG. Figure 1 shows a typical farfield image of the pure KB beam without the waveguide (b) compared to a farfield image obtained after aligning the waveguide in the focus (c). As the figure shows, the divergence increased, reflecting the smaller source size, and the illumination exhibited fewer high-frequency artefacts. Due to the waveguide geometry and absorption in the cladding of the waveguide, the total flux was reduced by approximately two orders of magnitude.

\section{References}

1. Pfeiffer, F., Weitkamp, T., Bunk, O. \& David, C. Phase retrieval and differential phase-contrast imaging with low-brilliance X-ray sources. Nat. Phys. 2, 258-261; DOI: 10.1038/nphys265 (2006).

2. Schulz, G. et al. High-resolution tomographic imaging of a human cerebellum: comparison of absorption and grating-based phase-contrast. J. R. Soc. Interface 7, 1665-1676 (2010).

3. Kitchen, M. J. et al. Phase contrast x-ray imaging of mice and rabbit lungs: a comparative study. Brit. J. Radiol. 78, 1018-1027; DOI: $10.1259 /$ bjr/13024611 (2005).

4. Bravin, A., Coan, P. \& Suortti, P. X-ray phase-contrast imaging: from pre-clinical applications towards clinics. Phys. Med. Biol. 58, R1 (2013).

5. Larabell, C. A. \& Nugent, K. A. Imaging cellular architecture with X-rays. Curr. Opin. Struct. Biol. 20, 623-631(2010).

6. Nugent, K. A. Coherent methods in the X-ray sciences. Adv. Phys. 59, 1-99 (2010).

7. Paganin, D. M. Coherent X-ray Optics (New York, Oxford University Press, 2006).

8. Schmahl, G., Rudolph, D., Niemann, B. \& Christ, O. Zone-plate X-ray microscopy. Q. Rev. Biophys. 13, 297-315 (1980).

9. Chien, C. et al. Imaging cells and sub-cellular structures with ultrahigh resolution full-field x-ray microscopy. Biotechnol. Adv. 31, 375-386 (2013)

10. Pfeiffer, F., Kottler, C., Bunk, O. \& David, C. Hard X-Ray Phase Tomography with Low-Brilliance Sources. Phys. Rev. Lett. 98, 108105; DOI: 10.1103/PhysRevLett.98.108105 (2007)

11. David, C., Nohammer, B., Solak, H. H. \& Ziegler, E. Differential x-ray phase contrast imaging using a shearing interferometer. Appl. Phys. Lett. 81, 3287-3289; DOI: 10.1063/1.1516611 (2002).

12. Momose, A., Takeda, T., Itai, Y. \& Hirano, K. Phase-contrast X-ray computed tomography for observing biological soft tissues. Nat. Med. 2, 473-475; DOI: 10.1038/nm0496-473 (1996).

13. Thibault, P. Algorithmic methods in diffraction microscopy. Ph.D. thesis, (University Microfilms International, Ann Arbor, Mich. (2008). 
14. Dierolf, M. et al. Ptychographic X-ray computed tomography at the nanoscale. Nature 467, 436-439; DOI: 10.1038/nature09419 (2010).

15. Cloetens, P. et al. Holotomography: Quantitative phase tomography with micrometer resolution using hard synchrotron radiation x rays. Appl. Phys. Lett. 75, 2912-2914 (1999).

16. Nugent, K. A., Gureyev, T. E., Cookson, D. F., Paganin, D. \& Barnea, Z. Quantitative Phase Imaging Using Hard X Rays. Phys. Rev. Lett. 77, 2961-2964 (1996).

17. Wilkins, S. W., Gureyev, T. E., Gao, D., Pogany, A. \& Stevenson, A. W. Phase-contrast imaging using polychromatic hard X-rays. Nature 384, 335-338; DOI: 10.1038/384335a0 (1996).

18. Liu, H. et al. Effects of missing low-frequency information on ptychographic and plane-wave coherent diffraction imaging. Appl. Opt. 52, 2416-2427 (2013).

19. Zanette, I. et al. Holotomography versus x-ray grating interferometry: A comparative study. Appl. Phys. Lett. 103, (2013).

20. Pinzer, B. et al. Imaging brain amyloid deposition using grating-based differential phase contrast tomography. NeuroImage 61, 1336-1346 (2012).

21. Schropp, A. Experimental Coherent X-Ray Diffractive Imaging : Capabilities and Limitations of the Technique. Ph.D. thesis, Universität Hamburg (2008).

22. Williams, G. J. et al. Fresnel Coherent Diffractive Imaging. Phys. Rev. Lett. 97, 025506 (2006).

23. Nishino, Y., Takahashi, Y., Imamoto, N., Ishikawa, T. \& Maeshima, K. Three-Dimensional Visualization of a Human Chromosome Using Coherent X-Ray Diffraction. Phys. Rev. Lett. 102, 018101; DOI: 10.1103/PhysRevLett.102.018101 (2009).

24. Jiang, H. et al. Quantitative 3D imaging of whole, unstained cells by using X-ray diffraction microscopy. PNAS 107, 11234-11239 (2010).

25. Nelson, J. et al. High-resolution x-ray diffraction microscopy of specifically labeled yeast cells. PNAS 107, 7235-7239 (2010).

26. Guizar-Sicairos, M. et al. Phase tomography from x-ray coherent diffractive imaging projections. Opt. Express 19, 21345-21357 (2011).

27. Giewekemeyer, K. et al. X-ray propagation microscopy of biological cells using waveguides as a quasipoint source. Phys. Rev. A 83, 023804; DOI: 10.1103/PhysRevA.83.023804 (2011).

28. Turner, L. et al. X-ray phase imaging: Demonstration of extended conditions for homogeneous objects. Opt. Express 12, 2960 2965 (2004).

29. Allen, L. \& Oxley, M. Phase retrieval from series of images obtained by defocus variation . Opt. Commun. 199, 65-75 (2001).

30. Krenkel, M., Bartels, M. \& Salditt, T. Transport of intensity phase reconstruction to solve the twin image problem in holographic x-ray imaging. Opt. Express 21, 2220-2235 (2013).

31. Bartels, M., Krenkel, M., Haber, J., Wilke, R. N. \& Salditt, T. X-ray holographic imaging of hydrated biological cells in solution. Phys. Rev. Lett. 114, 048103 (2015).

32. Bartels, M. et al. Low-dose three-dimensional hard x-ray imaging of bacterial cells. Opt. Nanoscopy 1, 1-10 (2012).

33. Cloetens, P., Mache, R., Schlenker, M. \& Lerbs-Mache, S. Quantitative phase tomography of Arabidopsis seeds reveals intercellular void network. PNAS 103, 14626-14630 (2006).

34. Olendrowitz, C. et al. Phase-contrast x-ray imaging and tomography of the nematode Caenorhabditis elegans. Phys. Med. Biol. 57, 5309 (2012).

35. Bartels, M., Hernandez, V. H., Krenkel, M., Moser, T. \& Salditt, T. Phase contrast tomography of the mouse cochlea at microfocus x-ray sources. Appl. Phys. Lett. 103, 083703 (2013).

36. Moosmann, J. et al. High-resolution $\mathrm{x}$-ray phase-contrast tomography from single-distance radiographs applied to developmental stages of xenopus laevis. J. Phys.: Conf. Ser. 425, 192003 (2013).

37. Mokso, R., Cloetens, P., Maire, E., Ludwig, W. \& Buffiere, J. Nanoscale zoom tomography with hard $\mathrm{x}$ rays using Kirkpatrick-Baez optics. Appl. Phys. Lett. 90, 144104 (2007).

38. Bleuet, P. et al. A hard x-ray nanoprobe for scanning and projection nanotomography. Rev. Sci. Instrum. 80, (2009).

39. Moreira, A. P. \& Hogaboam, C. M. Macrophages in allergic asthma: Fine-tuning their pro- and anti-inflammatory actions for disease resolution. J. Interferon Cytokine Res. 31, 485-491 (2011).

40. Bang, B.-R. et al. Alveolar macrophages modulate allergic inflammation in a murine model of asthma. Exp. Mol. Med. 43, 275-280; DOI: 10.3858/emm.2011.43.5.028 (2011).

41. Yang, M., Kumar, R. K., Hansbro, P. M. \& Foster, P. S. Emerging roles of pulmonary macrophages in driving the development of severe asthma. J. Leukocyte Biol. 91, 557-569 (2012).

42. Balhara, J. \& Gounni, A. S. The alveolar macrophages in asthma: a double-edged sword. Mucosal Immunol. 5, 605-609; DOI: 10.1038/mi.2012.74 (2012).

43. Dullin, C. et al. Functionalized synchrotron inline phase contrast ct: a novel approach for simultaneous quantification of structural alterations and localization of barium labelled alveolar macrophages within mouse lung samples. J. Synchrotron Rad. 22, 143-155; DOI: 10.1107/S1600577514021730 (2015).

44. Mbawuike, I. N. \& Herscowitz, H. B. MH-S, a murine alveolar macrophage cell line: morphological, cytochemical, and functional characteristics. J. Leukoc. Biol. 46, 119-127 (1989).

45. Groso, A., Abela, R. \& Stampanoni, M. Implementation of a fast method for high resolution phase contrast tomography. Opt. Express 14, 8103-8110 (2006).

46. Zabler, S., Cloetens, P., Guigay, J.-P., Baruchel, J. \& Schlenker, M. Optimization of phase contrast imaging using hard x rays. Rev. Sci. Instrum. 76, 073705 (2005).

47. van Heel, M. \& Schatz, M. Fourier shell correlation threshold criteria. J. Struct. Biol. 151, 250-262 (2005).

48. Markus, M. A. et al. Non-invasive optical imaging of eosinophilia during the course of an experimental allergic airways disease model and in response to therapy. PLoS ONE 9, e90017; DOI: 10.1371/journal.pone.0090017 (2014).

49. Somogyi, A. et al. ID22: a multitechnique hard X-ray microprobe beamline at the European Synchrotron Radiation Facility. J. Synchrotron Rad. 12, 208-215; DOI: 10.1107/S0909049504030882 (2005).

50. Martinez-Criado, G. et al. Status of the hard x-ray microprobe beamline id22 of the european synchrotron radiation facility. J. Synchrotron Rad. 19, 10-18; DOI: 10.1107/S090904951104249X (2012).

51. Krüger, S. P. et al. Sub-15 nm beam confinement by twocrossed x-ray waveguides. Opt. Express 18, 13492-13501 (2010).

52. Bartels, M. Cone-beam $\mathrm{x}$-ray phase contrast tomography of biological samples Optimization of contrast, resolution and field of view. Ph.D. thesis, Universität Göttingen (2013).

\section{Acknowledgments}

We thank Michael Sprung and the P10 team for assistance in operating the P10 GINIX instrument, and we thank Peter Cloetens and his team for help with the experiments at the ID22Ni beamline. We also thank Bärbel Heidrich, Julia Scherber, and Sarah Greco for help with cell culture and animal work. Funding provided by the DFG collaborative research centre SFB 755 'Nanoscale Photonic Imaging' and 
the German Ministry of Education and Research (BMBF-Verbundforschung, Grant No. 05K10MGA) is gratefully acknowledged.

\section{Author Contributions}

F. A., T. S., C. D. and M. K. designed the experiments. A. M. prepared the samples. M. K., M. B. and T. S. performed the experiments. M. K. performed the analysis and prepared the figures. M. K. and T. S. wrote the main manuscript text. All authors reviewed the manuscript.

\section{Additional Information}

Supplementary information accompanies this paper at http://www.nature.com/srep

Competing financial interests: The authors declare no competing financial interests.

How to cite this article: Krenkel, M. et al. Phase-contrast zoom tomography reveals precise locations of macrophages in mouse lungs. Sci. Rep. 5, 9973; doi: 10.1038/srep09973 (2015).

(c) (i) This work is licensed under a Creative Commons Attribution 4.0 International License. The images or other third party material in this article are included in the article's Creative Commons license, unless indicated otherwise in the credit line; if the material is not included under the Creative Commons license, users will need to obtain permission from the license holder to reproduce the material. To view a copy of this license, visit http://creativecommons.org/licenses/by/4.0/ 


\title{
Dendritic Polyglycerolsulfate Near Infrared Fluorescent (NIRF) Dye Conjugate for Non-Invasively Monitoring of Inflammation in an Allergic Asthma Mouse Model
}

\author{
Stefania Biffi ${ }^{1,2 * 9}$, Simeone Dal Monego ${ }^{39}$, Christian Dullin $^{4}$, Chiara Garrovo $^{1,2}$, Berislav Bosnjak ${ }^{5}$, \\ Kai Licha ${ }^{6}$, Pia Welker ${ }^{6}$, Michelle M. Epstein ${ }^{5}$, Frauke Alves ${ }^{7}$
}

1 Cluster in Biomedicine (CBM scrl), Optical Imaging Laboratory, Trieste, Italy, $\mathbf{2}$ Institute for Maternal and Child Health, IRCCS Burlo Garofolo, Trieste, Italy, $\mathbf{3}$ Cluster in Biomedicine (CBM scrl), Bioinformatics, Trieste, Italy, 4 Department of Diagnostic Radiology, University Medical Center Göttingen, Göttingen, Germany, $\mathbf{5}$ Department of Dermatology, Division of Immunology, Allergy and Infectious Diseases, Experimental Allergy, Medical University of Vienna, Vienna, Austria, $\mathbf{6}$ Mivenion GmbH, Berlin, Germany, 7 Department of Hematology and Oncology, University Medical Center Göttingen, Germany

\begin{abstract}
Background: Non-invasive in vivo imaging strategies are of high demand for longitudinal monitoring of inflammation during disease progression. In this study we present an imaging approach using near infrared fluorescence (NIRF) imaging in combination with a polyanionic macromolecular conjugate as a dedicated probe, known to target L- and P-selectin and C3/C5 complement factors.

Methodology/Principal Findings: We investigated the suitability of dendritic polyglycerol sulfates (dPGS), conjugated with a hydrophilic version of the indocyanine green label with 6 sulfonate groups (6S-ICG) to monitor sites of inflammation using an experimental mouse model of allergic asthma. Accumulation of the NIRF-conjugated dPGS (dPGS-NIRF) in the inflamed lungs was analyzed in and ex vivo in comparison with the free NIRF dye using optical imaging. Commercially available smart probes activated by matrix metalloproteinase's (MMP) and cathepsins were used as a comparative control. The fluorescence intensity ratio between lung areas of asthmatic and healthy mice was four times higher for the dPGS in comparison to the free dye in vivo at four hrs post intravenous administration. No significant difference in fluorescence intensity between healthy and asthmatic mice was observed 24 hrs post injection for dPGS-NIRF. At this time point ex-vivo scans of asthmatic mice confirmed that the fluorescence within the lungs was reduced to approximately $30 \%$ of the intensity observed at 4 hrs post injection.
\end{abstract}

Conclusions/Significance: Compared with smart-probes resulting in a high fluorescence level at 24 hrs post injection optical imaging with dPGS-NIRF conjugates is characterized by fast uptake of the probe at inflammatory sites and represents a novel approach to monitor lung inflammation as demonstrated in mice with allergic asthma.

Citation: Biffi S, Dal Monego S, Dullin C, Garrovo C, Bosnjak B, et al. (2013) Dendritic Polyglycerolsulfate Near Infrared Fluorescent (NIRF) Dye Conjugate for NonInvasively Monitoring of Inflammation in an Allergic Asthma Mouse Model. PLoS ONE 8(2): e57150. doi:10.1371/journal.pone.0057150

Editor: Henrik Einwaechter, Klinikum rechts der Isar der TU München, Germany

Received January 27, 2012; Accepted January 21, 2013; Published February 21, 2013

Copyright: (c) 2013 Biffi et al. This is an open-access article distributed under the terms of the Creative Commons Attribution License, which permits unrestricted use, distribution, and reproduction in any medium, provided the original author and source are credited.

Funding: The funders (European Commission through an FP7- IAPP Marie Curie Action - (GA 230739)) had no role in study design, data collection and analysis, decision to publish, or preparation of the manuscript.

Competing Interests: Stefania Biffi, Simeone dal Monego and Chiara Garrovo are employees of Cluster in Biomedicine (CBM Scrl). CBM offers services in different research areas, spanning from genomics and bioinformatics, to optical imaging and advanced microscopy. CBM is also actively involved in research collaborations within the framework of european and national projects, as well as project management and grant writing support. Kai Licha and Pia Welker are employees of Mivenion $\mathrm{GmbH}$. Mivenion is a life-science company focusing on personalized medicine for patients with inflammatory and autoimmune diseases. A network of academia and industry partners is supporting Mivenion. This does not alter the authors' adherence to all the PLOS ONE policies on sharing data and materials. The authors from Academia (Goettingen and Vienna) have declared that no competing interests exist.

*E-mail: stefania.biffi@cbm.fvg.it

9 These authors contributed equally to this work.

\section{Introduction}

Currently, NIRF imaging is a common technology in preclinical studies that obtains functional information in vivo over time for assessment of antibody binding, protein expression, enzyme activities, cell tracking etc. [1-3]. Optical imaging provides relatively inexpensive and non-harmful methods and is preferred over other imaging methods used in preclinical research and drug development, such as PET and SPECT that are more complex to perform. However, the penetration depth of typically up to $4 \mathrm{~cm}$ in the near infrared range (NIR) limits its clinical application to endoscopic techniques and structures beneath the skin or fluorescence guided surgery [4]. Crucial for the success of in vivo NIRF imaging will be the development of dedicated NIRF probes for distinct targets of molecular events characterizing different diseases. So far, these probes, based on their mechanisms of targetdetection can be divided into four groups: passive probes to image areas with increased blood supply [5], target-specific fluorescent probes which are directed against molecular and/or diseasespecific markers [6], fluorescent labels to track injected fluores- 
cence stained cells [7], and application of smart probes activated by enzymes for the detection of molecular events [8].

NIRF imaging in lung disease models has remained challenging due to the high scattering nature of the lung and its comparable deep location. Recent application of novel non-invasive imaging technologies in mouse models of asthma has enabled functional and longitudinal in vivo monitoring of disease, validation of novel biomarkers, and direct tracking of immune cells within tissues. Novel methods for in vivo monitoring of lung inflammation in mice include the utilization of smart-probes activated by MMPs or cathepsin, enzymes known to be involved in lung inflammation [9-11].

Airway inflammation is a central component of asthma that consists of edema, cellular infiltration, particularly of eosinophils, neutrophils, activated $\mathrm{T}$ lymphocytes and mast cells, increased airway secretions, and deposition of excess collagen. Therefore mouse models of asthma present attractive tools for evaluating probes suitable for in vivo molecular imaging of lung inflammation $[12,13]$.

Using a model of allergen-induced lung inflammation, we applied fluorescence imaging in combination with near-infrared (NIR) fluorescently-labeled dendritic polyglycerol sulfates (dPGS), a class of compounds that selectively bind to mediators of inflammatory processes such as L- and P-selectin and C3/C5 complement factors $[14,15]$. The role of selectin-ligand interactions in allergic asthma is well established, making them an attractive target for visualization of inflammation [16-19]. For example, reduced airway hyperresponsiveness in asthma in LSelectin-deficient mice has been reported [19]. Furthermore, studies show that dPGS is transported into inflammatory cells e.g. in activated mononuclear cells [20,21]. Generally, dPGS consists of a highly branched (dendritic) polyglycerol core, which due to the large amount of hydroxyl end groups enables high functionalization. In our case, sulfate groups were generated from the hydroxyl groups, thereby creating the highly charged, polyanionic dPGS compound (Figure 1). dPGS acts via a multivalent binding mechanism mimicking naturally occurring selectin ligands [20], with a clearly demonstrated dependence of the binding affinity from molecular weight and degree of sulfation [15,21]. Sulfation of the hydroxyl groups in the polymer established a multivalent polyanionic entity with high affinity for L- and P-selectin [22]. Anti-inflammatory property of dPGS in much higher concentrations has been reported to occur as a result of a multivalent interaction enabled by the multitude of sulfate groups. For instance, binding of dPGS to L-selectin on leukocytes and Pselectin on inflamed vascular endothelium reduces leukocyte extravasation by shielding the adhesion molecule [22]. Additionally, inhibition of C5a generation inhibits leukocyte chemotaxis $[14,22]$.

The compound used herein has a core molecular weight of about $6000 \mathrm{Da}$, imparting high binding affinity of the respective polysulfate [21] and at the same time having a reasonable molecular weight range for sufficient distribution and excretion [20], as well as chemical derivatization in order to conjugate NIRF dyes to the polymeric entity. The aim of the present study was to assess the capacity of dPGS conjugated with a near infrared fluorescent (NIRF) dye related to indocyanine green (dPGS-NIRF) to detect inflammatory sites in lungs by NIRF optical imaging analysis in a mouse model of asthma and to compare dPGS-NIRF to the commercially available smart-probes MMPSense and ProSense.

\section{Results}

OVA-immunization and challenge-induced allergic inflammation and extensive mucus hypersecretion in the lungs, and elevated serum OVA-specific IgG1

Allergic asthma inflammation and mucus hypersecretion in mice was induced by two intraperitoneal injections and subsequent intranasal challenges with OVA. Figure 2 illustrates lung histology from $\mathrm{H} \& \mathrm{E}$ and PAS-stained lung sections of asthmatic and healthy control mice. H\&E staining revealed that no inflammatory infiltrates were present in lungs from healthy mice (Figure 2A). In contrast, immunized mice had dense inflammatory infiltrates containing predominantly eosinophils, as well as macrophages and lymphocytes surrounding blood vessels, and large and small airways (Figure 2B). The extent of allergic inflammation was evaluated by assessing the total surface area and location of leukocyte infiltration in lung sections (Figure 2C). Mice with allergic inflammation have histological scores of 5.2 \pm 0.4 (dPGSNIRF group) and $4.4 \pm 0.3$ (dye group) compared to healthy controls with $0.5 \pm 0.3$ (dPGS-NIRF group) to $0.8 \pm 0.3$ (dye group), demonstrating that diseased mice have lung inflammation affecting more than two thirds of the examined lung sections with infiltrates present in the hilum extending to the lung periphery.

To assess mucus hypersecretion, adjacent lungs sections were stained with PAS. As expected, only rare mucus producing cells were detected in the central airways of healthy control mice (Figure 2D), whereas numerous mucus producing cells were observed in asthmatic mice (Figure 2E). Histological evaluation revealed that asthmatic mice have histological scores for mucus overproduction of 2.8 \pm 0.4 (dPGS-NIRF group) and 2.5 \pm 0.5 (dye group) compared to healthy controls with $0.3 \pm 0.3$ (dPGS-NIRF group) to $0.2 \pm 0.3$ (dye group) (Figure $2 \mathrm{~F}$ ), indicating that mucus hypersecretion extended to the periphery of the diseased lungs.

We also tested serum OVA-specific Th2-isotype antibody titres. While no OVA-specific antibodies in sera were detected before immunization with OVA, high titres $(\geq 1: 7812500)$ of OVAspecific IgG1 were detected in all OVA-sensitizated and challenged mice (results not shown), further supporting presence of allergic immune responses in both investigated groups.

\section{Allergic asthma can be successfully visualized by combination of dPGS-NIRF probe and in vivo optical imaging}

To visualize allergic inflammation in vivo, we injected dPGSNIRF and the control dye i.v. into the tail vein at $72 \mathrm{hrs}$ after last OVA challenge, when we expected that allergic inflammation in the lung is at its peak. Asthmatic and healthy mice were imaged at 4 and $24 \mathrm{hrs}$ post dPGS-NIRF or unconjugated NIRF dye injection as control.

Figures 3 and 4 illustrate the distribution of the control dye and dPGS-NIRF, respectively, after $4 \mathrm{hrs}$ in the thoracic area of asthmatic in comparison to healthy mice. A slight increase of fluorescent signal was recorded after injection of control dye in asthmatic mice in comparison to healthy mice (Figure 3A). In order to localize the dPGS-NIRF probe within inflamed lung region we applied fluorescence microscopy in combination with immunofluorescence staining of macrophages by the use of an antibody against F4/80, a $160 \mathrm{kDa}$ cell surface glycoprotein that is widely expressed on mature tissue macrophages. As shown in Figure 3B a higher amount of macrophages was clearly detectable in lungs of asthmatic mice in comparison to healthy controls. The Control dye was not detected in lung sections of asthmatic mice using fluorescence microscopy (Figure 3B). In contrast, higher 
<smiles>CO[N+](=O)[O-]</smiles>

Figure 1. Chemical structure of dPGS-NIRF. The chemical structure indicates the linker structure and connection to the dye (approx. 3 dyes per polymer). Please note that the polymer is not depicted in original molecular weight, but is shown only as principle sketch.

doi:10.1371/journal.pone.0057150.g001
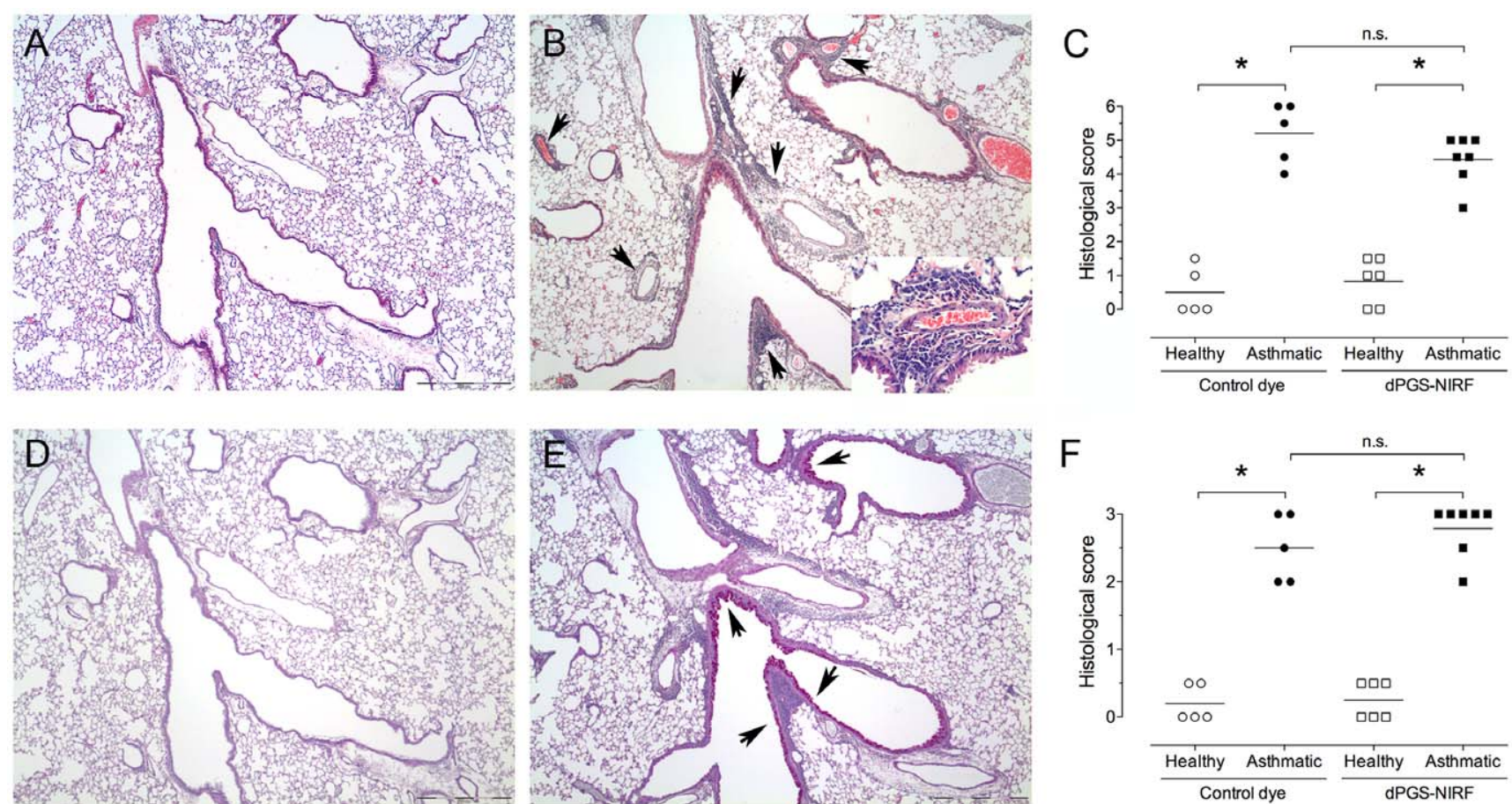

Figure 2. Allergic inflammation and mucus hypersecretion in the lungs of asthmatic, but not control mice. Lungs were harvested 76 hrs after the final ovalbumin (OVA) challenge meaning 4 hrs post i.v. probe injection. Representative H\&E (A and B) and PAS (D and E) stained photomicrographs of lungs from healthy $(A$ and $D$ ) or asthmatic mice ( $B$ and $E$ ) are shown (magnification $40 \times$, inset $400 \times$ ). (B) Arrows indicate inflammation, and in (E) arrows indicate mucus hypersecretion. Allergic inflammation (C) and mucus hypersecretion (F) scores in H\&E and PAS stained lung sections, respectively, of healthy (open symbols) or asthmatic mice (filled symbols). Each symbol represents individual mice ( $n=5-6$ for healthy groups and $n=5-7$ for asthmatic groups), and line represents group mean. One-way ANOVA followed by Tukey's multiple comparison test (*P<0.05) was used to compare differences between groups.

doi:10.1371/journal.pone.0057150.g002 
fluorescence intensity was detected in the thoracic region of asthmatic mice $4 \mathrm{hrs}$ post dPGS-NIRF probe injection (Figure 4A). Moreover, fluorescence microscopy of lung sections of asthmatic mice confirmed dPGS-NIRF probe localization in areas where F4/80 stained macrophages could be detected, which demonstrated that dPGS-NIRF accumulates especially in the inflamed region of lungs of the pathological model (Figure 4B).

Fluorescence signals obtained with in vivo imaging were quantified and intensity ratios were calculated as described in the Material and Methods. As depicted in Figure 5A, at 4 hrs post injection of control dye, we observed a slight increase in fluorescence signal in asthmatic mice when compared to healthy mice (increase in average $R I_{\text {Dye }}(4 \mathrm{~h}) \sim 11 \%$, p-value $=0.047$ ), most probably due to an increase in the vascular flow in the inflamed lungs. In contrast, dPGS-NIRF increased the fluorescence signal in the thorax of asthmatic mice dramatically, as seen by an average $R I_{d P G S}(4 \mathrm{~h}) \sim 44 \%$ with $\mathrm{p}$-value $=0.004$. Moreover, a direct comparison of the contrast (RI) between dPGS-NIRF and free dye in the asthmatic mice revealed a $30 \%$ higher $R I_{d P G S}(4 \mathrm{~h})$ than $R I_{\text {Dye }}(4 \mathrm{~h})$ (p-value $\left.=0.005\right)$ at this time point. At $24 \mathrm{hrs}$ post dPGS-NIRF injection, fluorescence signals over the lung areas of healthy and asthmatic mice were not longer distinguishable (average $R I_{d P G S}(24 \mathrm{~h}) \quad$ difference $\sim 8 \%, \quad \mathrm{p}$-value $=0.162$ ) (Figure 5B). In vitro analysis of serum binding of ICG as well as of 6S-ICG demonstrate that ICG completely binds to serum proteins (23), whereas less than $40 \%$ of $6 \mathrm{~S}-\mathrm{ICG}$ was bound to serum proteins (data not shown).

\section{Ex vivo optical imaging confirmed the in vivo results}

To confirm the in vivo imaging findings immediately after the last imaging, we imaged the lungs ex-vivo using an Optix MX2 system. Ex-vivo imaging avoids autofluorescence of other organs and absorption and scattering within the body and fur. This increases both specificity and sensitivity of probe detection. In accordance to the in vivo results, we found a significant difference between the fluorescence intensity within the lungs of asthmatic and healthy mice $4 \mathrm{hrs}$ post injection of the dPGS-NIRF conjugate (difference of $R I_{d P G S}^{e x-v i v o}(4 \mathrm{~h}) \sim 65 \%$, p-value $=0.009$ ), but not control dye (difference of $R I_{D y e}^{\text {ex-vivo }}(4 \mathrm{~h}) \sim 18 \%$, pvalue $=0.127)($ Figure $6 \mathrm{~A}$ and $6 \mathrm{~B})$. At $24 \mathrm{hrs}$ post administration of dPGS-NIRF, the observed fluorescence intensity over the lungs was reduced to about $30 \%$ of the intensity measured 4 hrs post injection. Moreover, the difference in fluorescence intensity between healthy and asthmatic mice dropped down to $\sim 10 \%$ and was not significant (difference of $\operatorname{RI}_{d P G S}^{\text {ex }- \text { vivo }}(24 \mathrm{~h}) \sim 10 \%$, pvalue $=0.323)($ Figure 6B).

\section{Comparison of dPGS-NIRF with commercially available smart-probes}

Commercially available smart-probes ProSense and MMPSense, activated by cathepsins and MMPs, respectively, were used for imaging lung inflammation [9]. Both smart-probes were injected at $72 \mathrm{hrs}$ after the last OVA challenge and imaged after $24 \mathrm{hrs}$, according to probe manufacturer's recommendations. The intensity difference from the thoracic region between healthy and asthmatic mice was $\sim 27 \%$ (p-value $=0.013$ ) after administration of ProSense and $\sim 83 \%$ after injection of MMPSense but with no statistical significance $(p$-value $=0.093)($ Table 1$)$.

\section{Discussion}

In this study, we present a novel approach for functional in-vivo imaging utilizing a dendritic polyglycerolsulfate conjugated to a NIRF dye related to ICG (dPGS-NIRF) in combination with optical imaging to monitor sites of inflammation in the lung by applying an experimental model of allergic asthma [12].

We successfully demonstrated that the applied dPGS-NIRF probe accumulates to inflammatory sites within the lung already $4 \mathrm{hrs}$ after probe administration. The results show a significant four times stronger contrast of the fluorescence intensity of the dPGS-NIRF probe compared to the free dye in lungs of asthmatic in comparison to healthy mice. At this time point fluorescence

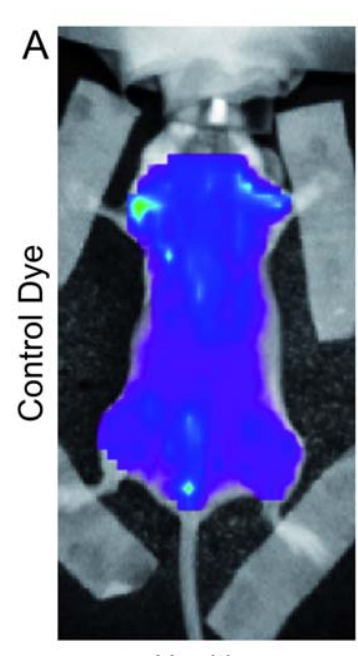

Healthy

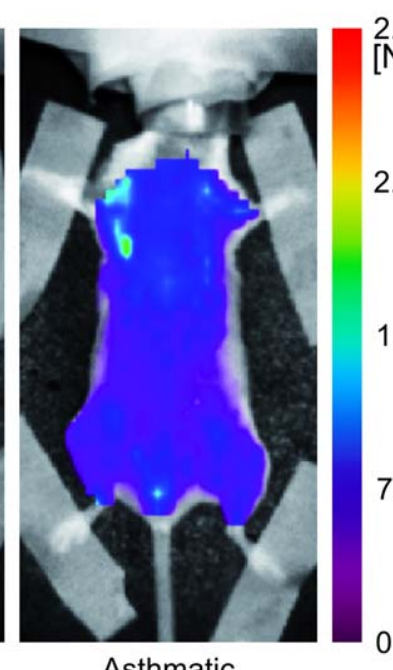

Asthmatic

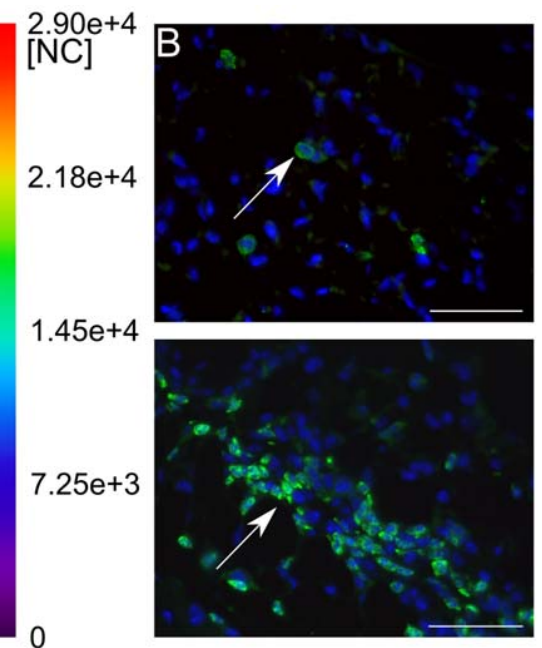

Figure 3. In vivo distribution of free dye (indocyanine green) 4 hours post probe injection and 76 hours post last OVA challenge. Panel A: whole body fluorescence intensity distribution of a representative healthy and asthmatic mouse displayed in normalized counts [NC]. Panel B: Fluorescence microscopy images of F4/80 stained macrophages and DAPI stained cell nuclei of lungs isolated from asthmatic and healthy mice injected with the NIRF labeled control dye and sacrificed 4 hrs post injection demonstrate no fluorescent control dye. F4/80 expression on macrophages are depicted in green, cell nucleus in blue, control dye was not detected (bar $=50 \mu \mathrm{m})$. In the healthy model few macrophages have been detected with respect to the asthmatic mouse, where cluster of cells are visible (see white arrows indicating macrophages). In both samples no unconjugated NIRF dye 6S-ICG has been visualized.

doi:10.1371/journal.pone.0057150.g003 


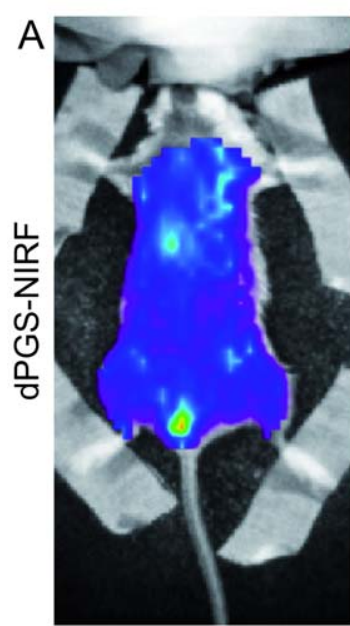

Healthy

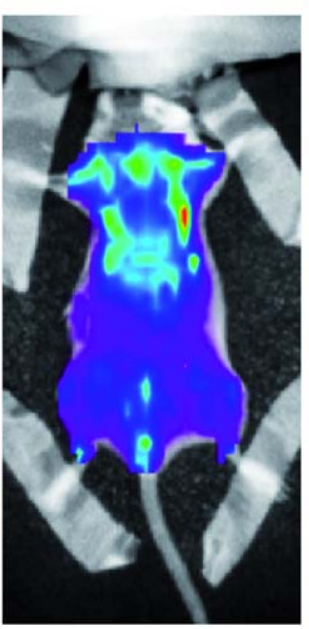

Asthmatic

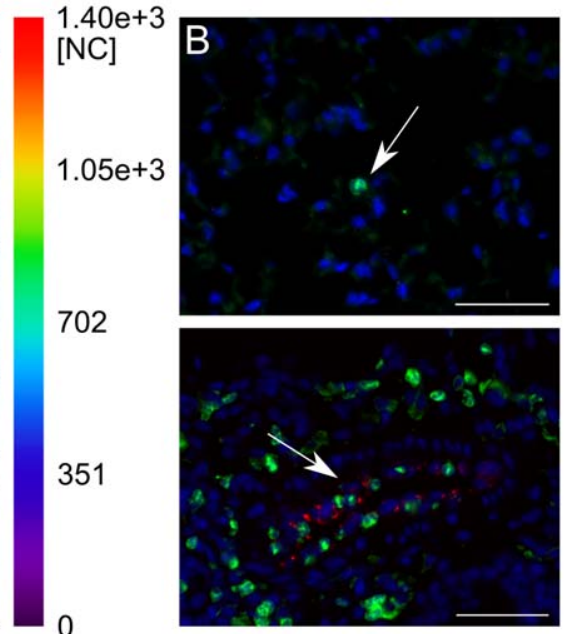

Figure 4. In vivo distribution of dPGS-NIRF 4 hours post probe injection and 76 post last OVA challenge. Panel A: whole body fluorescence intensity distribution in a representative healthy and asthmatic mouse displayed in normalized counts [NC]. Stronger fluorescence intensity over the lung area of the asthmatic mouse can be seen. Panel B Fluorescence microscopy images of F4/80 stained macrophages and DAPI stained cell nuclei of lungs isolated from asthmatic and healthy mice injected with dPGS-NIRF and sacrificed 4 hrs post injection. F4/80 expression on macrophages are depicted in green, cell nucleus in blue, dPGS-NIRF displayed in red (bar $=50 \mu \mathrm{m})$. In the healthy model, few macrophages and no probe localization have been detected. In the asthmatic mouse, cluster of macrophages are detectable (see white arrows) and the dPGS-NIRF probe was visualized in the same region of macrophages.

doi:10.1371/journal.pone.0057150.g004

microscopy confirmed the localization of the dPGS-NIRF probe within the inflamed lungs in areas where F4/80 stained macrophages could be detected and histology demonstrated the presence of inflammatory infiltrates in more than two thirds of the examined lung sections. Therefore, dPGS-NIRF is suitable to monitor inflamed lungs by NIRF imaging.

Furthermore, at $4 \mathrm{hrs}$ post injection, the calculated $R I_{e x}(4 h)$ was significantly lower in asthmatic lungs after administration of free dye. These results indicate that a specific target in the model appears to be involved. dPGS-NIRF exerts high-affinity binding to positively charged protein motifs e.g. P- and L-selectin as well as to C3/C5 complement factors [22]. The selectivity is demonstrated by the very low affinity for E-selectin compared to P and Lselectin, which dPGS bind to with nanomolar affinity in vitro $[20,22]$. Furthermore, dPGS accumulates in inflamed tissue by a not yet understood cellular uptake mechanism into macrophages and endothelial cells, but not into lymphocytes. This was shown for example by fluorescence microscopy of liver tissue specimens after dPGS-NIRF application that depicted accumulation in rat liver macrophages (Kupffer cells) and of A549 tumor cells as well

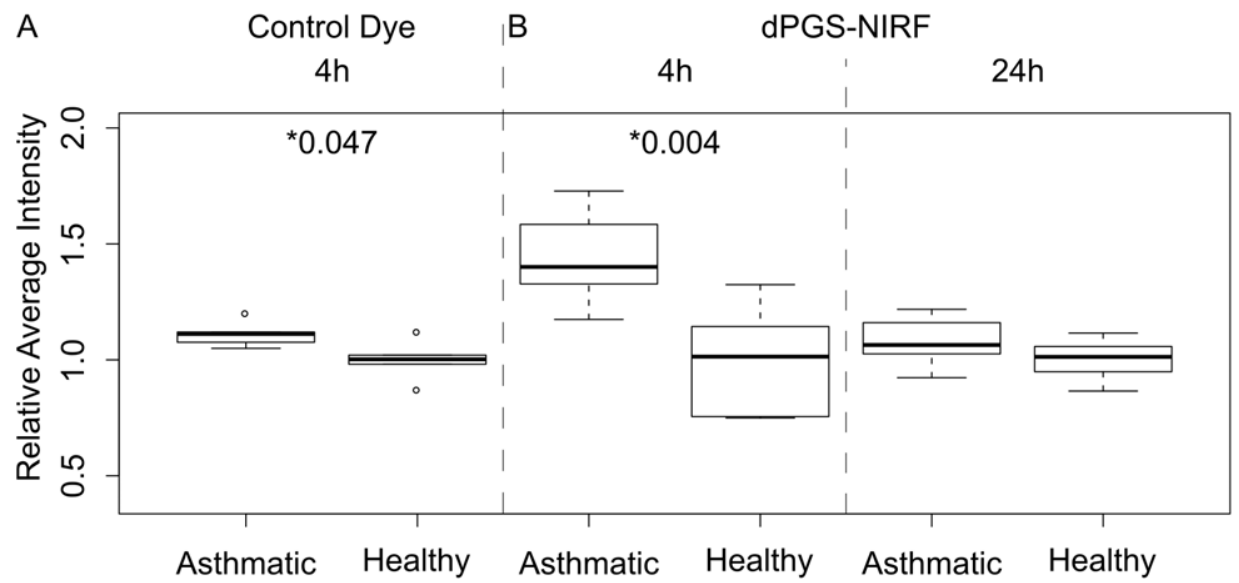

Figure 5. Quantification of in vivo imaging results of dPGS-NIRF and pure dye. Box plots of ratios of average fluorescence intensity over the lung area compared with the mean value of each control group respectively are reported for asthmatic and healthy mice. Mice treated with free dye $4 \mathrm{hrs}$ post injection showed a slight increase in fluorescence signal in asthmatic mice $(n=5)$ when compared to healthy mice $(n=5$; increase in average $\sim 11 \%$, $p$-value $=0.047$, panel A). Mice treated with dPGS-NIRF probe 4 hrs post injection (healthy $n=6$, asthmatic $n=6$ ) showed an increased fluorescence signal in the thorax in asthmatic mice (increase in average $\sim 44 \%$ with $p$-value $=0.004$, panel $B$ left side). At 24 hrs post injection fluorescence signals over the lung areas of healthy $(n=5)$ and asthmatic mice $(n=10)$ shown no difference (difference $\sim 8 \%, p$-value $=0.162$, panel $B$ right side). Both control dye and dPGS-NIRF probe were injected $72 \mathrm{hrs}$ after last aerosol challenge. Note, intensity ratios were used to compare probes with different brightness, therefore the box plots are depicted in the same scale. doi:10.1371/journal.pone.0057150.g005 


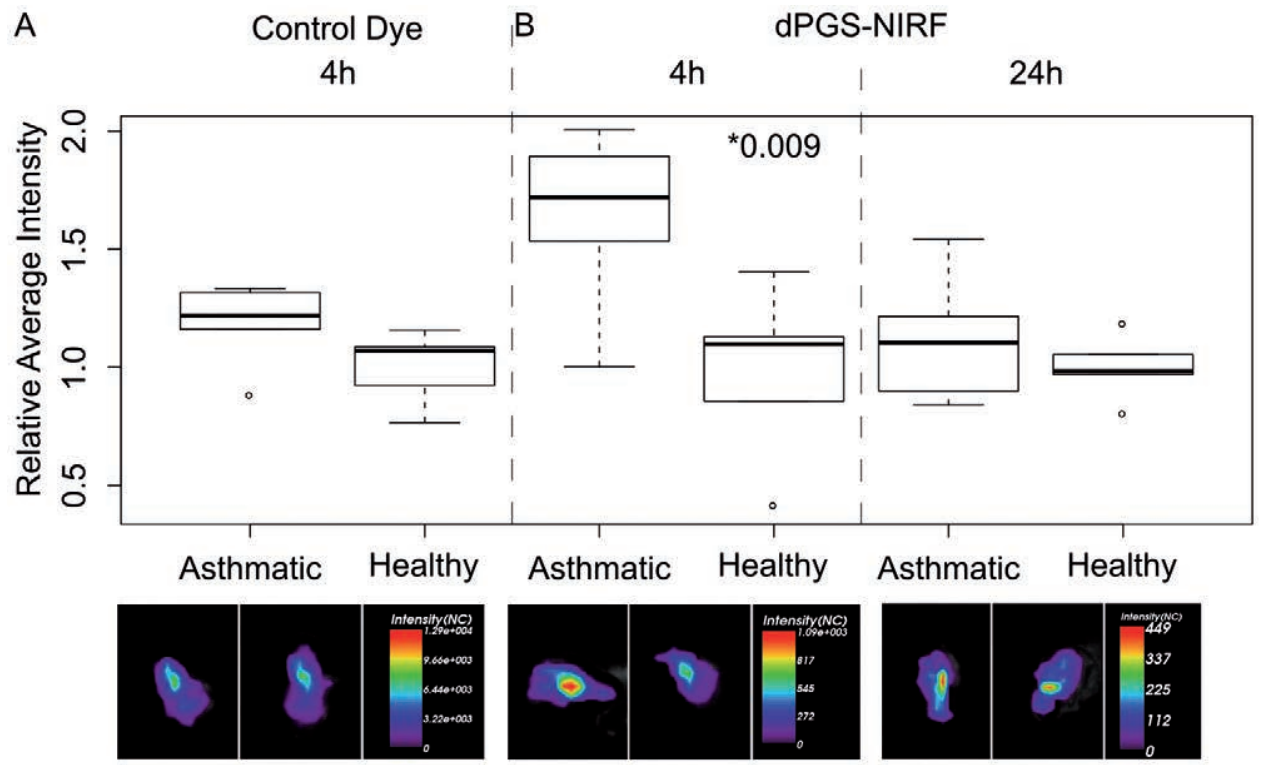

Figure 6. Ex vivo imaging results of dPGS-NIRF and pure dye. Box plots of ratios of average fluorescence intensity over the explanted lungs compared with the mean value of each control group respectively are reported for asthmatic and healthy mice treated with free dye 4 hrs post injection (panel A), and treated with dPGS-NIRF probe $4 \mathrm{hrs}$ (panel B left side) and 24 hrs (panel B right side) post injection. The corresponding fluorescence intensity images of representative lungs are given at the bottom of each box plot. A significant difference between the fluorescence intensity within the lungs of asthmatic $(n=5)$ and healthy mice $(n=5)$ was observed 4 hrs post injection of the dPGS-NIRF conjugate (difference of $\sim 65 \%$, $p$-value $=0.009$ ), but not of the control dye (healthy $n=5$, asthmatic $n=5$; difference of $\sim 18 \%$, $p$-value $=0.127$ ). Both control dye and dPGSNIRF probe were injected $72 \mathrm{hrs}$ after last aerosol challenge. Note, intensity ratios were used to compare probes with different brightness, therefore the box plots are depicted in the same scale.

doi:10.1371/journal.pone.0057150.g006

as of activated, LPS-stimulated mononuclear cells, both demonstrating accumulation of dPGS [22].

The underlying chemical structure of the polymer in published studies [22] is based on a polyglycerol core of $6000 \mathrm{Dal}$, whereas different dyes were attached to the polymer, such as a visible cyanine dye or a NIRF dye in a ratio of approx. 1 dye per polymer, yielding identical selectin-binding properties. The conjugate used herein employs the same polymer, but a more hydrophilic indocyanine dye with 6 sulfonate groups (6S-ICG) added to the fluorophore structure. Coupling to the dPGS could be achieved at a dye-to-polymer ratio of 3 without signs of aggregation known to be induced by more lipophilic indocyanine dyes in bioconjugates, as described in [20].

Previously, studies show also inflammation-specific imaging with dPGS-NIRF in an animal model of collagen induced rheumatoid arthritis using the preceding conjugate with a lipophilic indocyanine green label. Comparable to our study, the authors demonstrated a fast and selective uptake of the probe with a 3.5 fold higher fluorescence difference between healthy and diseased joints and a signal peak at $1 \mathrm{hr}$ after probe administration. Together with a rough estimation of a blood half-life of shorter than $1 \mathrm{hr}$ by employing the eye fluorescence as a provisional solution to monitor blood kinetics, they postulate targeting mechanisms not yet fully understood [20] whereby dPGS-NIRF binds to mediators of inflammation.

Interestingly, the high contrast between the fluorescence intensity of dPGS-NIRF in the asthmatic and healthy groups was not observed after $24 \mathrm{hrs}$. This might be explained in part by shedding of P- and L-selectins from the cell surface after binding of dPGS-NIRF [23]. Bound dPGS-NIRF probes will be removed from the cells resulting in the reduction of fluorescence intensity to background after 24 hrs.

The MMPSense and ProSense probes, which are activated in the presence of inflammation-associated enzymes such as cathepsin and MMP's that are present in the lungs during allergen challenge are successfully used by others, for example to detect lung inflammation and rapidly screen for new drug effects [9-11] as well as to visualize colon adenomas [8]. Similar to our study,

Table 1. Calculated average fluorescence intensity ratios $R I_{e x}(t)$ between healthy and asthmatic mice after injection of control dye, dPGS-NIRF, or two commercially available probes: ProSense and MMPSense.

\begin{tabular}{|c|c|c|c|c|c|}
\hline \multirow[b]{2}{*}{ measurement time (hours) } & \multirow{2}{*}{$\frac{\text { Control Dye }}{4}$} & \multicolumn{2}{|l|}{ dPGS-NIRF } & \multirow{2}{*}{$\begin{array}{l}\text { ProSense } \\
24\end{array}$} & \multirow{2}{*}{$\begin{array}{l}\text { MMPSense } \\
24\end{array}$} \\
\hline & & 4 & 24 & & \\
\hline in vivo & $1.11 \pm 0.06(0.047)$ & $1.45 \pm 0.20(0.004)$ & $1.08 \pm 0.10(0.162)$ & $1.27 \pm 0.02(0.013)$ & $1.83 \pm 0.50(0.093)$ \\
\hline ex vivo & $1.18 \pm 0.18(0.127)$ & $1.65 \pm 0.35(0.009)$ & $1.10 \pm 0.22(0.323)$ & n.d. & n.d. \\
\hline
\end{tabular}

Results are shown as mean calculated average fluorescence intensity ratios \pm standard deviation, while statistical significance between each pair of control and asthmatic mice is given by p-value for the Welch-T-Test in brackets. Legend: n.d. - not done.

doi:10.1371/journal.pone.0057150.t001 
Cortez-Retamozo et al. demonstrated fluorescence differences between asthmatic lungs and healthy controls by applying the same amount of MMPSense or ProSense however by using fiberoptic bronchoscopy and fluorescence molecular tomography (FMT) [9]. Others also reported that the in vivo profile of cysteine protease activation was depicted by FMT in a mouse model of acute airway inflammation by LPS-induction [10,11].

These smart probes exhibit slower kinetics due to their activation mechanism, demonstrating maximal fluorescence intensity within the lungs about $24 \mathrm{hrs}$ after probe injection [9]. The application of these enzymatically activated probes is often hampered by the fact that despite a dramatic increase of their fluorescence intensity over inflammatory areas, the activated probes do not remain at the site of interest for very long and instead produce a strong liver signal due to their excretion pathway.

In conclusion, we present a novel in vivo NIRF imaging probe for detection of inflammatory reactions within the lungs of mice, as demonstrated in mice with allergic inflammation, by utilizing a dendritic polyglycerolsulfate NIRF dye conjugate known to bind to selectins and complement factors. The greater fluorescence intensity of dPGS-NIRF in inflammation of mice with allergic asthma in combination with rapid kinetics makes dPGS-NIRF a powerful probe candidate to monitor inflammation processes and responses to therapy in experimental mouse models of lung disease.

\section{Materials and Methods}

\section{Mice}

Female BALB/c mice (4- to 6-weeks old) were purchased from Charles River and maintained with ad libitum food and water. All the experimental procedures were performed in compliance with the guidelines of European (86/609/EEG) and Italian (D.L.116/ 92) as well as German laws and were approved by the Italian Ministry of University and Research and the Administration of the University Animal Facility, Trieste, as well as by the administration of Lower Saxony, Germany.

\section{Synthesis of a dendritic polyglycerol sulfates NIR dye conjugate probe (dPGS-NIRF)}

dPGS was synthesized by anionic polymerization of glycidol and subsequent sulfation using SO3/pyridinum complex according to Türk and colleagues [14]. Conjugation of dPGS to an NIRF dye (based on indocyanine green chromophore; derivative with reactive group for conjugation) are described [20] elsewhere. Briefly, the polyglycerol intermediate was reacted with an aliphatic linker chain followed by the sulfation reaction. To this linker, a novel NIRF dye (6S-ICG propargyl; mivenion $\mathrm{GmbH}$ ) was conjugated followed by high-performance liquid chromatography (HPLC) purification yielding dPGS-NIRF with a mean dye-topolymer ratio of 3 and an average molecular weight of $19000 \mathrm{Da}$. The degree of sulfonation was $85 \%$ (elementary analysis) and the polydispersity index (PDI) within 1.6-1.8 (measured for the polyglycerol intermediate using GPC). The dye used herein is a hydrophilic version of the previously described indocyanine green label, with 4 additional sulfonate groups in the molecule resulting in a 6 -fold sulfonated entity of maximal hydrophilicity for this type of NIR fluorophore. The chemical structure is depicted in Figure 1. Absorption maxima in PBS were 710 and $795 \mathrm{~nm}$, fluorescence emission maximum $810 \mathrm{~nm}$. Unconjugated NIRF dye (6S-ICG molecular weight $\sim 1700 \mathrm{~g} / \mathrm{mol}$, free carboxylic acid instead of linkage to polymer) served as control probe in the in vivo experiments.

\section{Mouse Model of Acute Allergic Asthma}

Mice were sensitized intraperitoneally (i.p.) at day 0 and day 21 with $10 \mu \mathrm{g}$ ovalbumin (OVA) dissolved in $200 \mu \mathrm{l}$ PBS. At day 28 and day 29 mice were treated intranasal (i.n.) with a solution of $100 \mu \mathrm{g}$ OVA/50 $\mu \mathrm{l}$ PBS/mouse. Healthy age and gender matched $\mathrm{BALB} / \mathrm{c}$ mice served as controls. Histology of $\mathrm{H} \& \mathrm{E}$ stained lung sections was performed at $76 \mathrm{hrs}$ post last challenge

\section{Optical Imaging Scan}

48 mice were examined by optical imaging (Table 2). Mice were shaved over the lung area prior to the scanning procedure in order to reduce scattering of the signal from fur. Throughout all imaging sessions, mice were anesthetized with vaporized isoflurane at 1.8-2 volume $\%$ as described [2]. The anesthetized mice were placed inside an Optix MX2 acquisition system (Advanced Research Technologies, Montreal, Canada) and gently fixed on a heated block $\left(37^{\circ} \mathrm{C}\right)$ for the entire duration of data acquisition.

All in vivo analyses were preceded by native scans of the mice prior to NIRF probe injection to provide a base line for later analysis. At $72 \mathrm{hrs}$ after the last OVA challenge, mice were injected intravenously (i.v.) via the tail with $100 \mu \mathrm{l}$ of one of the following: dPGS-NIRF (2.6 nmol, polymer/dye $=1 / 3)$, free NIRF dye $(3.6 \mathrm{nmol}), 100 \mu \mathrm{l}(5 \mathrm{nmol})$ of either MMPSense (MMPSen$\mathrm{se}^{\circledR}$, Perkin Elmer) or ProSense (ProSense ${ }^{\circledR}$, Perkin Elmer), all dissolved in $0.9 \% \mathrm{NaCl}$. The amount of injected dPGS-NIRF and NIRF solutions was calculated based on the weaker fluorescence signal of dye in the conjugate than in the unconjugated control dye. Exact numbers of animals in each group are shown in Table 2.

\section{In vivo and ex vivo Optical Imaging}

Animals with acute asthma and wild type controls were scanned at 4 and 24 hrs post i.v. dPGS-NIRF or NIRF dye administration. For the MMPSense and ProSense, scans were performed $24 \mathrm{hrs}$ after probe administration. According to the supplier (PerkinElmer), this time point constitutes the peak activation of these probes [9]. All in vivo data was acquired by using the small-animal timedomain Optix MX2 preclinical NIRF-imager (Advanced Research Technologies, Montreal, CA), equipped with four pulsed laser diodes and a time correlated single photon counting detector [24]. This system works in reflection mode applying a raster acquisition scheme, measuring and analyzing fluorescence response to pulsed excitation for each excitation spot by creating fluorescence photon time of flight histograms. In all imaging experiments applying the dPGS-NIRF and control dye, a $785 \mathrm{~nm}$ pulsed laser diode with a repetition frequency of $80 \mathrm{MHz}$ was used whereas for the MMPSense and ProSense studies a $670 \mathrm{~nm}$ pulsed laser diode with a repetition frequency of $80 \mathrm{MHz}$ was applied. Fluorescence emission was accordingly collected with an $800 \mathrm{~nm}$ long pass filter for dPGS-NIRF and control dye and a $700 \mathrm{~nm}$ long pass filter for both MMPSense and ProSense to block the excitation light. Two-dimensional regions of interest (ROIs) were selected, and laser power, integration time (repetition time of the excitation per raster point), and scan step size were optimized according to the emitted signal. Prior to probe application, mice were scanned to obtain background images. These background signal intensities recorded with the baseline image for each animal before the injection of the probe was subtracted from each post injection image. At the end of the last imaging session, 4 and $24 \mathrm{hrs}$ after dPGS-NIRF/NIRF dye i.v. injection, animals were sacrificed and ex vivo optical imaging of the explanted lungs was performed. To calculate the total lung fluorescence intensity (Ilung) in each scan, fluorescence intensities were normalized with the laser power used for excitation and summed up in ROI's 
Table 2. Experimental design of optical imaging biodistribution study.

\begin{tabular}{|c|c|c|c|c|c|}
\hline \multirow{2}{*}{$\begin{array}{l}\text { Measurement time } \\
\text { (hours) }\end{array}$} & \multirow{2}{*}{$\begin{array}{l}\text { Control Dye } \\
4\end{array}$} & \multicolumn{2}{|c|}{ dPGS-NIRF } & \multirow{2}{*}{$\begin{array}{l}\text { ProSense } \\
24\end{array}$} & \multirow{2}{*}{$\begin{array}{l}\text { MMPSense } \\
24\end{array}$} \\
\hline & & 4 & 24 & & \\
\hline Healthy mice (number) & 5 & 6 & 5 & 2 & 2 \\
\hline Asthmatic mice (number) & 5 & 6 & 10 & 3 & 4 \\
\hline
\end{tabular}

encompassing the whole organ. Lungs were then preserved in formalin for histological analysis.

\section{Image processing}

Image analysis was done using OptiView (2.02.00), the proprietary software developed for the Optix device. All data sets of mice receiving dPGS-NIRF, free NIRF dye control as well MMPSense and ProSense were normalized for different excitation laser power and variations of the used integration time and therefore expressed in normalized counts [NC], an arbitrary unit. Average fluorescence intensity was calculated within a region of interest covering the whole lung for every sample $(\mathrm{x})$ and time point (t) as $\bar{I}(x, t)$ and subtracted by the base line intensity within the same region $\bar{I}_{b g}(x)$. To remove the influence of different brightness of all applied probes, ratios $R I_{e x}(x, t)$ between the average intensity of the sample (x) and the mean average intensity of the control group for each experiment $(\mathrm{ex})$ and time point $(\mathrm{t})$ were calculated and denominated as $R I_{e x}(x, t)$.

$$
R I_{e x}(x, t)=\frac{\bar{I}(x, t)-\bar{I}_{b g}(x)}{N_{c n t}^{-1} \sum \bar{I}(x, t)}
$$

$R I_{e x}(x, t)$ can be interpreted as contrast or probability to distinguish asthma mice from controls at certain time points and was therefore used for comparison of the different studies and statistical calculations.

\section{Histological analysis of lung inflammation}

Following ex vivo image analysis performed $76 \mathrm{hrs}$ after the last ovalbumin challenge, tissue samples were fixed in $10 \%$ buffered formalin and embedded in paraffin. To evaluate allergic lung inflammation, $3 \mu \mathrm{m}$ thick lung sections containing main stem bronchi were stained with hematoxylin and eosin (H\&E). A blinded grading of the slides was done to evaluate the intensity and extent of inflammation according to our semi-quantitative scoring system. For intensity of inflammation: 0 - no inflammatory infiltrates; 1 - inflammatory infiltrates in central airways; 2 inflammatory infiltrates extending to middle third of lung parenchyma; and 3 - inflammatory infiltrates extending to periphery of the lungs. For extent of inflammation: $0-$ no inflammatory infiltrates; 1 -inflammatory infiltrates present in one third of lung surface; 2 - inflammatory infiltrates spreading up to two thirds of lung surface; 3 - inflammatory infiltrates present in more than two thirds of lung surface. Data are presented as histological score calculated as the sum of intensity and extent of inflammation for each sample. For detection of mucus-containing cells in lung tissue, adjacent $3 \mu \mathrm{m}$ sections containing main stem bronchi from each lung specimen were stained with periodic acidSchiff (PAS) and counter stained with hematoxylin. Slides were examined blinded for the treatment and mucus overproduction was scored as: Grade 0 - no mucus producing cells in airways; Grade 1 - few mucus producing cells in central airways; Grade 2 mucus producing cells detected in middle airways; and Grade 3 mucus producing cells extending to respiratory bronchioles. In borderline cases, an intermediate grade was used ( $0.5 ; 1.5$ or 2.5), extending the scoring to a total of seven grades.

\section{Serum OVA-specific immunoglobulin}

For the measurement of OVA-specific immunoglobulin (Ig) G1, ELISA plates were coated with OVA at $10 \mu \mathrm{g} / \mathrm{ml}$ overnight at $4^{\circ} \mathrm{C}$. The plates were washed and blocked with $2 \%$ bovine serum albumin in PBS with $0.05 \%$ Tween 20 for $2 \mathrm{hrs}$ at RT. Then sera were titrated onto the plates and incubated for $24 \mathrm{hrs}$ at $4^{\circ} \mathrm{C}$ before washing. Plates were incubated for an additional $2 \mathrm{hrs}$ at $4^{\circ} \mathrm{C}$ with biotinylated anti-IgGl (Southern biotechnology associates Inc., Birmingham, AL, USA) detection mAb, followed by incubation with streptavidin horseradish peroxidase (Southern biotechnology) for $1 \mathrm{~h}$ at RT. Plates were washed and incubated with TMB substrate solution (100 $\mu \mathrm{l} /$ well, BD OptEIATM, Becton Dickinson Biosciences) for $10 \mathrm{~min}$ at $\mathrm{RT}$. The reaction was stopped with $100 \mu \mathrm{l}$ of $0.18 \mathrm{M} \mathrm{H} 2 \mathrm{SO} 4$ and the plates were measured at $450 \mathrm{~nm}$.

\section{In vitro analysis of serum binding}

The serum binding of $6 \mathrm{~S}-\mathrm{ICG}$ was determined in vitro by incubation with pooled human serum (PAA) with dye concentration of $5 \mu \mathrm{g} / \mathrm{ml}$ [25]. The sample was placed in a Centriprep micropartition unit NWML $30 \mathrm{kDa}$ (Milipore, Billerica, USA), and centrifuged at $5000 \mathrm{~g}$ for $20 \mathrm{~min}$. The protein-bound 6S-ICG and the free dye in the ultrafiltrate was quantified spectrophotometrically (Beckman Coulter, USA).

\section{Fluorescence microscopy}

Detection of injected dPGS-NIRF probe or unconjugated NIRF dye $6 \mathrm{~S}-\mathrm{ICG}$ in lungs of control and asthmatic mice was carried out by fluorescence microscopy. In order to correlate NIRF fluorescence signals from the probes to inflammatory sites, lungs were counterstained with at anti-mouse F4/80 antibody. detecting macrophages Two-micrometer-thick sections were cut from paraffin blocks, the slides were first processed for avidin/biotin and protein blocking steps using xylol and decreasing alcohol concentration for deparaffination and rehydration and later incubated with the primary antibody rat anti-mouse F4/80 (AbD Serotec, Oxford, UK), dilution factor $1: 100$ at $4{ }^{\circ} \mathrm{C}$ overnight. After the incubation with the primary antibody, the samples were incubated with secondary biotinylated antibody goat anti-rat (BioLegend, San Diego, USA), dilution factor 1:200 at RT for 1 hour, and then with streptavidin- Alexa 555 (Molecular Probes, Life Technologies Corporation, USA) dilution factor 1:400 at RT for 1 hour. DAPI was diluted in the mounting media and used as nuclear counterstaining. Fluorescence was analyzed with a Zeiss Axiovert $200 \mathrm{M}$ inverted microscope (Carl Zeiss, 
Germany) equipped with a xenon lamp and a high sensitivity ORCA-AG digital camera (Hamamatsu, Japan). Data were acquired with AxioVs40 software (Carl Zeiss). Filter settings were as followed: DAPI: Ex: BP 365/25 (+/-12.5); FT 395; Em: BP 445/50 (+/-25); Cy7: BP 708/75 (+/-37.5); FT 757; BP 809/81 (+/-40.5); Alexa555: BP 546/12 (土6); FT 580 and LP 590 filter. Subsequent analyses were performed using the java-based image processing program ImageJ.

\section{Statistical Analysis}

Statistical verification of the differences of $R I_{e x}(x, t)$ between asthmatic and control mice for each experiment and time point was done using an unpaired Welch Two Sample t-test implemented in the PAST statistic software [26]. A p-value of less than 0.05 was considered significant.

Histological scores between groups were compared using Oneway ANOVA followed by Tukey's multiple comparison test.

\section{References}

1. Biffi S, Garrovo C, Macor P, Tripodo G, Zorzet S, et al. (2008) In vivo biodistribution and lifetime analysis of cy5.5-conjugated rituximab in mice bearing lymphoid tumor xenograft using time-domain near-infrared optical imaging. Mol Imaging 7: 272-282.

2. Agostinis C, Biffi S, Garrovo C, Durigutto P, Lorenzon A, et al. (2011) In vivo distribution of $\beta 2$ glycoprotein I under various pathophysiological conditions. Blood 118(15):4231-8.

3. Napp J, Mathejczyk JE, Alves F (2011) Optical imaging in vivo with a focus on paediatric disease: technical progress, current preclinical and clinical applications and future perspectives. Pediatr Radiol 41: 161-175.

4. Hutteman M, Choi HS, Mieog JSD, van der Vorst JR, Ashitate Y, et al. (2011) Clinical translation of ex vivo sentinel lymph node mapping for colorectal cancer using invisible near-infrared fluorescence light. Ann. Surg. Oncol. 18: 10061014 .

5. Montet X, Figueiredo J-L, Alencar H, Ntziachristos V, Mahmood U, et al. (2007) Tomographic fluorescence imaging of tumor vascular volume in mice. Radiology 242: 751-758.

6. Dullin C, Zientkowska M, Napp J, Missbach-Guentner J, Krell H-W, et al. (2009) Semiautomatic landmark-based two-dimensional-three-dimensional image fusion in living mice: correlation of near-infrared fluorescence imaging of Cy5.5-labeled antibodies with flat-panel volume computed tomography. Mol Imaging 8: 2-14.

7. Ushiki T, Kizaka-Kondoh S, Ashihara E, Tanaka S, Masuko M, et al. (2010) Noninvasive tracking of donor cell homing by near-infrared fluorescence imaging shortly after bone marrow transplantation. PLoS ONE 5: e11114.

8. Clapper ML, Hensley HH, Chang W-CL, Devarajan K, Nguyen MT, et al. (2011) Detection of colorectal adenomas using a bioactivatable probe specific for matrix metalloproteinase activity. Neoplasia 13: 685-691.

9. Cortez-Retamozo V, Swirski FK, Waterman P, Yuan H, Figueiredo JL, et al. (2008) Real-time assessment of inflammation and treatment response in a mouse model of allergic airway inflammation. J Clin. Invest. 118: 4058-4066.

10. Ntziachristos V (2009) Optical imaging of molecular signatures in pulmonary inflammation. Proc Am Thorac Soc 6: 416-418.

11. Haller J, Hyde D, Deliolanis N, de Kleine R, Niedre M, et al. (2008) Visualization of pulmonary inflammation using noninvasive fluorescence molecular imaging. J Appl. Physiol. 104: 795-802.

12. Epstein MM (2004) Do mouse models of allergic asthma mimic clinical disease? Int. Arch. Allergy Immunol. 133: 84-100.
GraphPad Prism (v.5.00, GraphPad Software, San Diego, CA) was used for data analysis.

\section{Acknowledgments}

This work was carried out at the University Medical Center Goettingen (Germany) with excellent support of Rosi Streich, Sarah Greco and Andrea Markus. We also want to acknowledge to Joanna Napp who helped in the fluorescence acquisition of the images.

\section{Author Contributions}

Conceived and designed the experiments: FA SB SDM MME BB. Performed the experiments: SB SDM CD CG BB. Analyzed the data: SB SDM CD MME BB. Contributed reagents/materials/analysis tools: KL PW. Wrote the paper: FA MME SB CD SDM.

13. Jannasch K, Missbach-Guentner J, Alves F (2009) Using in vivo imaging for asthma. Drug Discovery Today: Disease Models 6: 129-135.

14. Türk H, Haag R, Alban S (2004) Dendritic polyglycerol sulfates as new heparin analogues and potent inhibitors of the complement system. Bioconjug. Chem. 15: $162-167$.

15. Weinhart M, Gröger D, Enders S, Dernedde J, Haag R (2011) Synthesis of dendritic polyglycerol anions and their efficiency toward L-selectin inhibition. Biomacromolecules 12: 2502-2511.

16. Banerjee ER (2011) Triple selectin knockout (ELP-/-) mice fail to develop OVA-induced acute asthma phenotype. J Inflamm (Lond) 8: 19

17. Tang ML, Fiscus LC (2001) Important roles for L-selectin and ICAM-1 in the development of allergic airway inflammation in asthma. Pulm Pharmacol Ther 14: $203-210$.

18. Keramidaris E, Merson TD, Steeber DA, Tedder TF, Tang ML (2001) Lselectin and intercellular adhesion molecule 1 mediate lymphocyte migration to the inflamed airway/lung during an allergic inflammatory response in an animal model of asthma. J Allergy Clin. Immunol. 107: 734-738.

19. Royce SG, Lee M, Tang MLK (2010) The contribution of L-selectin to airway hyperresponsiveness in chronic allergic airways disease. J Asthma Allergy 3: 917

20. Licha K, Welker P, Weinhart M, Wegner N, Kern S, et al. (2011) Fluorescence Imaging with Multifunctional Polyglycerol Sulfates: Novel Polymeric near-IR Probes Targeting Inflammation. Bioconjug. Chem. 22: 2453-2460.

21. Reichert S, Welker P, Calderón M, Khandare J, Mangoldt D, et al. (2011) Sizedependant cellular uptake of dendritic polyglycerol. Small 7: 820-829.

22. Dernedde J, Rausch A, Weinhart M, Enders S, Tauber R, et al. (2010) Dendritic polyglycerol sulfates as multivalent inhibitors of inflammation. Proc. Natl. Acad. Sci. U.S.A. 107: 19679-19684.

23. Hafezi-Moghadam A, Thomas KL, Prorock AJ, Huo Y, Ley K (2001) L-selectin shedding regulates leukocyte recruitment. J Exp. Med. 193: 863-872.

24. Ma G, Gallant P, McIntosh L (2007) Sensitivity characterization of a timedomain fluorescence imager: eXplore Optix. Appl Opt 46: 1650-1657.

25. Gootz TD, Subashi TA, Lindner DL (1988) Simple spectrophotometric assay for measuring protein binding of penem antibiotics to human serum. Antimicrobial Agents and Chemotherapy 32: 159-163.

26. Hammer Ø, Harper D, Ryan P (2001) PAST: Paleontological Statistics Software Package for Education and Data Analysis. Palaeontological Association 22. 


\title{
Non-Invasive Optical Imaging of Eosinophilia during the Course of an Experimental Allergic Airways Disease Model and in Response to Therapy
}

\author{
M. Andrea Markus ${ }^{1 *}$, Christian Dullin ${ }^{2}$, Miso Mitkovski $^{3}$, Eva Prieschl-Grassauer ${ }^{4}$, Michelle M. Epstein $^{5}$, \\ Frauke Alves ${ }^{1,6}$
}

1 Department of Haematology and Oncology, University Medical Center Göttingen, Göttingen, Germany, 2 Department of Diagnostic and Interventional Radiology, University Medical Center Göttingen, Göttingen, Germany, 3 Light Microscopy Facility, Max-Planck-Institute of Experimental Medicine, Göttingen, Germany, 4 Marinomed Biotechnologie $\mathrm{GmbH}$, Vienna, Austria, 5 Department of Dermatology, Division of Immunology, Allergy and Infectious Diseases, Experimental Allergy, Medical University of Vienna, Vienna, Austria, 6 Department of Molecular Biology of Neuronal Signals, Max-Planck-Institute of Experimental Medicine, Göttingen, Germany

\begin{abstract}
Background: Molecular imaging of lung diseases, including asthma, is limited and either invasive or non-specific. Central to the inflammatory process in asthma is the recruitment of eosinophils to the airways, which release proteases and proinflammatory factors and contribute to airway remodeling. The aim of this study was to establish a new approach to non-invasively assess lung eosinophilia during the course of experimental asthma by combining non-invasive near-infrared fluorescence (NIRF) imaging with the specific detection of Siglec-F, a lectin found predominantly on eosinophils.

Methodology/Principal Findings: An ovalbumin (OVA)-based model was used to induce asthma-like experimental allergic airway disease (EAAD) in BALB/c mice. By means of a NIRF imager, we demonstrate that $48 \mathrm{~h}-72 \mathrm{~h}$ after intravenous (i.v.) application of a NIRF-labeled anti-Siglec-F antibody, mice with EAAD exhibited up to 2 times higher fluorescence intensities compared to lungs of control mice. Furthermore, average lung intensities of dexamethasone-treated as well as beta-escintreated mice were 1.8 and 2 times lower than those of untreated, EAAD mice, respectively and correlated with the reduction of cell infiltration in the lung. Average fluorescence intensities measured in explanted lungs confirmed the in vivo findings of significantly higher values in inflamed lungs as compared to controls. Fluorescence microscopy of lung cryosections localized the i.v. applied NIRF-labeled anti-Siglec-F antibody predominantly to eosinophils in the peribronchial areas of EAAD lungs as opposed to control lungs.
\end{abstract}

Conclusion/Significance: We show that monitoring the occurrence of eosinophils, a prominent feature of allergic asthma, by means of a NIRF-labeled antibody directed against Siglec-F is a novel and powerful non-invasive optical imaging approach to assess EAAD and therapeutic response in mice over time.

Citation: Markus MA, Dullin C, Mitkovski M, Prieschl-Grassauer E, Epstein MM, et al. (2014) Non-Invasive Optical Imaging of Eosinophilia during the Course of an Experimental Allergic Airways Disease Model and in Response to Therapy. PLoS ONE 9(2): e90017. doi:10.1371/journal.pone.0090017

Editor: Phillip A. Stumbles, Murdoch University, Australia

Received November 14, 2013; Accepted January 30, 2014; Published February 25, 2014

Copyright: (C) 2014 Markus et al. This is an open-access article distributed under the terms of the Creative Commons Attribution License, which permits unrestricted use, distribution, and reproduction in any medium, provided the original author and source are credited.

Funding: The presented data is part of the P3AGI project (public private partnership for asthma imaging and genomics) funded by the European Commission through an FP7- IAPP Marie Curie Action (GA 230739). The funders had no role in study design, data collection and analysis, decision to publish, or preparation of the manuscript.

Competing Interests: The author Eva Prieschl-Grassauer has read the journal's policy and has the following conflicts: She works for a commercial company, Marinomed Biotechnologie GmbH. All other authors have declared that no competing interests exist. This does not alter their adherence to PLOS ONE policies on sharing data and materials.

*E-mail: andrea.markus@med.uni-goettingen.de

\section{Introduction}

Allergic asthma is a chronic inflammatory disease of the lungs, which is characterized by a variable degree of bronchial obstruction, airway hyperresponsiveness (AHR) and increased mucus production. With over 300 million people affected and this number growing steadily, asthma is still a major health issue. While mild to moderate asthma is relatively well controlled by glucocorticoid therapy [1], 5-10\% of asthmatics are difficult to treat with current therapies and warrant a continuing search for new drugs [2]. Similar to other complex and heterogeneous diseases, our understanding of asthma is slowed by the fact that both genetic as well as environmental factors contribute to its origin and progression, and by the variety of cellular and molecular pathways involved [3]. As a result, animal models, especially in mice, have been vital in improving our knowledge of asthma and the development and validation of novel treatments [4]. Many of the characteristic features of human atopic asthma can be seen in mouse models. For example, following allergen challenge, profound eosinophilic infiltration of lung tissue and airways, an increase of lymphocytes, neutrophils, and monocytes in the lungs, activation of alveolar macrophages and thickening of the airway epithelium with a marked goblet cell hyperplasia are all characteristics found in both humans and mice [5].

Until recently, preclinical animal studies, including the assessment of mouse EAAD, relied heavily on invasive or terminal 
procedures such as bronchoalveolar lavage (BAL) and histology of excised tissue. Latest improvements of imaging techniques such as PET, SPECT, MRI, CT and OCT have advanced non-invasive research on pulmonary diseases [6]. However, these techniques mainly facilitate the anatomical or structural assessment of the diseased lung and/or make use of radioactive agents. Optical imaging poses a great advantage, offering a rapid, cheap and easy methodology, which enables the detection of specific targets in a live animal over time [7]. Presently, near infrared fluorescent (NIRF) probes revealed several benefits over other fluorescent dyes because they minimize autofluorescence and penetrate deeper into the tissue [8]. Importantly, NIRF imaging lacks radioactivity and is therefore considered an alternative to nuclear imaging, the current gold standard for clinical functional imaging.

However, molecular imaging of lung diseases and in particular allergic asthma using fluorescence imaging (FI) is limited [6] and unspecific $[9,10]$. Only proteinases such as matrix metalloproteinases (MMPs) and cathepsins [9,10] as well as selectins [11] have so far been targeted with smart probes. However, such optical sensors may detect inflammation unrelated to eosinophilia. We took a new, more specific, approach to detect the allergic inflammatory process underlying asthma by targeting Siglec-F, a member of the family of Siglecs (sialic acid-binding, Ig-like lectins), which are single-pass transmembrane cell surface proteins found predominantly on leucocytes [12]. Siglec-F is a functional paralog of the human Siglec-8, both proteins preferentially recognising a sulphated glycan ligand closely related to sialyl Lewis $\mathrm{X}$, a common ligand for the selectin family of adhesion molecules [12]. Most siglec proteins undergo endocytosis, an activity tied to their roles in cell signaling and innate immunity. Both, the human as well as the mouse protein, are specifically upregulated on eosinophils during allergic inflammation, and therefore, represent specific markers for detection of allergic reactions, involving eosinophils. Induction of allergic lung inflammation in mice causes up-regulation of Siglec-F on blood and bone marrow eosinophils as well as quantitative up-regulation of endogenous Siglec-F ligands in the lung tissue and airways [13]. A weaker expression was also reported on macrophages [13,14]. The recruitment of eosinophils to the airways occurs at the late-phase of allergic inflammation and their release of proteases and proinflammatory factors is thought to eventually lead to airway remodeling [15]. Eosinophilia is, therefore, an excellent marker for monitoring allergic inflammation. It was recently shown that anti-Siglec-F alone or in combination with anti-CD45 can be used for the quantitative detection of eosinophils in mouse bone marrow and spleen and that the antigen profile $\mathrm{CD} 45(+) \operatorname{SiglecF}(+) \mathrm{CD} 11 \mathrm{c}(-)$ was the most effective at detecting eosinophils in the lung and correlated with direct morphometric counts under all conditions evaluated [16].

We show here, that 2D fluorescence reflectance imaging (FRI) in combination with a NIRF-labeled antibody to Siglec-F, is an ideal technique to specifically monitor allergic lung inflammation in vivo and to evaluate the effect of therapeutic drugs in preclinical studies. We observed significantly higher fluorescence signal intensities over the lungs in mice with EAAD than in controls. Moreover, we non-invasively demonstrate decreased Siglec-F fluorescence signals over the lung in response to two different asthma therapies, the commonly used glucocorticoid dexamethasone, as well as beta-escin, a new anti-inflammatory drug derived from Chinese horse chestnut seeds.

\section{Materials and Methods}

\section{Materials}

Monoclonal rat anti-mouse-Siglec-F antibody and a rat IgG2a isotype control were purchased from BD Biosciences (Heidelberg, Germany). Siglec-F antibody was custom-labeled by Squarix Biotechnology (Marl, Germany) with either Alexa Fluor 750 (dye to protein ratio 2.8) or Alexa Fluor 680 (dye to protein ratio 4.5) (Life Technologies GmbH, Darmstadt, Germany). These NIRFlabeled anti-Siglec-F antibodies are designated anti-SiglecF-750 and anti-SiglecF-680, respectively. IgG2a isotype control antibody was labeled with Alexa Fluor 750 (dye to protein ratio 3.1).

\section{Animals}

Pathogen-free female $\mathrm{BALB} / \mathrm{c}$ mice, $6-8$ weeks of age were purchased from Charles River Laboratories Inc. (Wilmington, MA). All animals were housed in a controlled environment with a regular 12-hour dark:light cycle, at $22^{\circ} \mathrm{C}$ and were fed laboratory chow (SAFE, Augy, France) and tap water ad libitum. Seven days before the imaging experiments, the food was switched to chlorophyll-free chow (Scientific Animal Food \& Engineering, Augy, France) to reduce autofluorescence from the stomach and gut of the animals.

\section{Induction of EAAD and Treatment Schedule}

As shown in Figure 1, BALB/c mice were immunized via intraperitoneal (i.p.) injection on days 0 and 21 with $10 \mu \mathrm{g}$ OVA (Sigma-Aldrich) in a volume of $0.2 \mathrm{ml}$ phosphate-buffered saline (PBS) per mouse. On days 28 and 29 post-immunization, mice were challenged intranasally (i.n.) by pipetting $25 \mu \mathrm{l}$ of $100 \mu \mathrm{g}$ OVA in PBS, into each nostril. Control mice received PBS only. Anti-Siglec-F-NIRF-labeled antibodies (12 $\mu \mathrm{g}$ in $150 \mu \mathrm{l}$ PBS) were given either 3 or 4 days post challenge by tail vein injection and mice were repeatedly scanned over a given period of time by

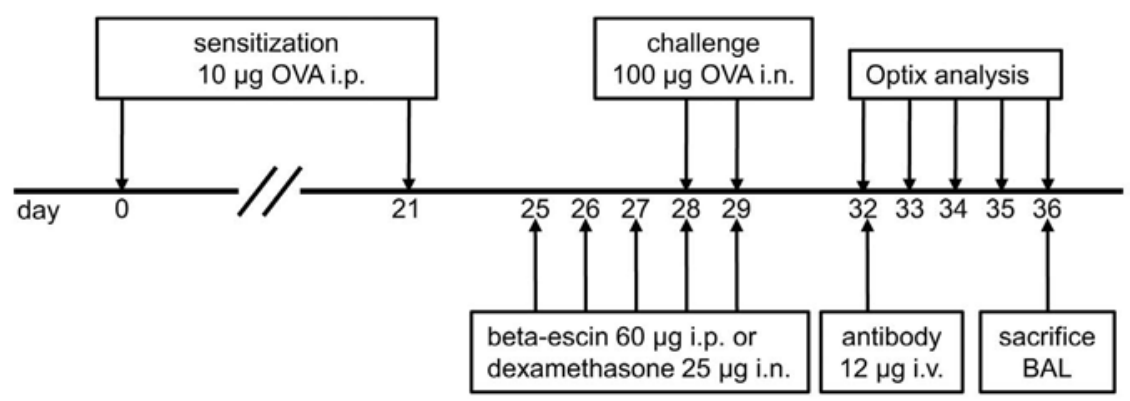

Figure 1. Induction of EAAD and treatment schedule. Schematic depiction of the experimental protocol used for the induction of EAAD, the application of treatments and the optical imaging performed. doi:10.1371/journal.pone.0090017.g001 
A

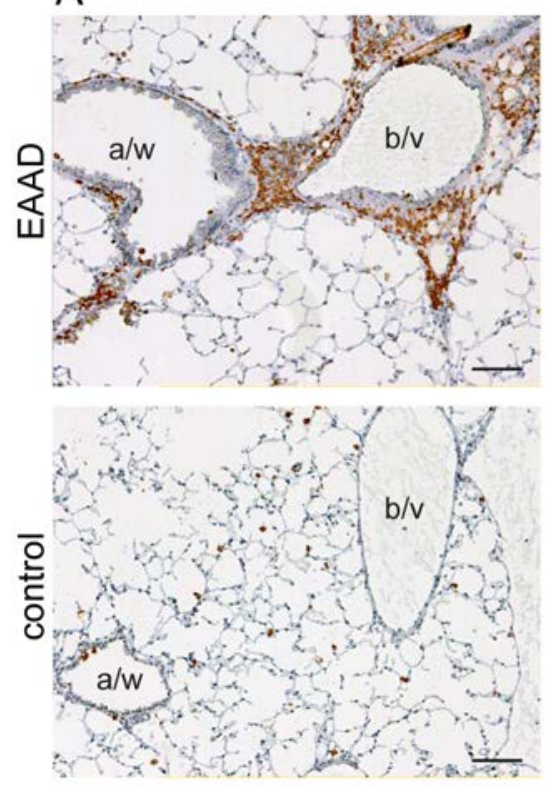

B

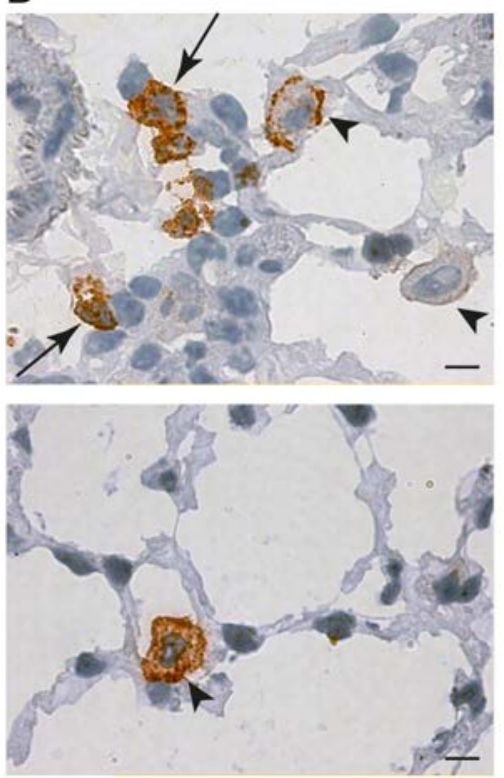

C

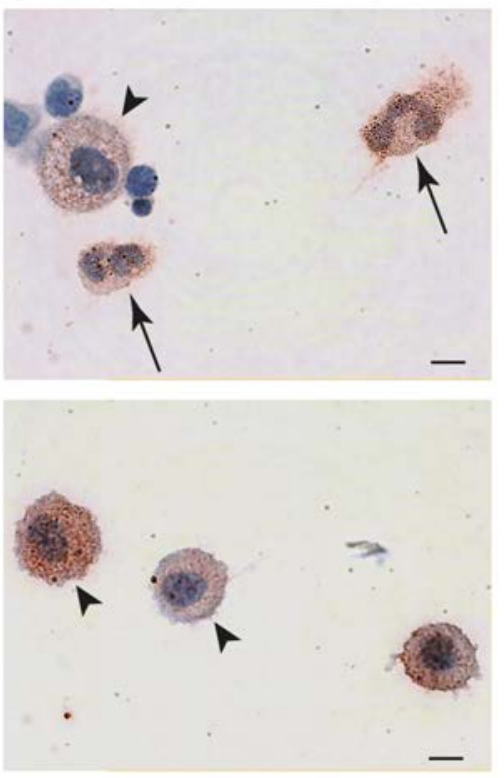

D
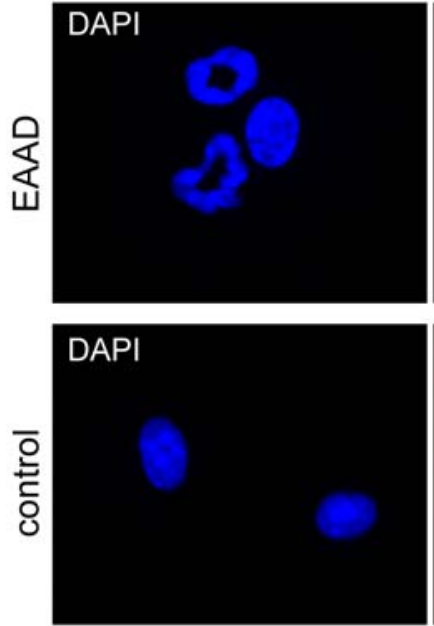

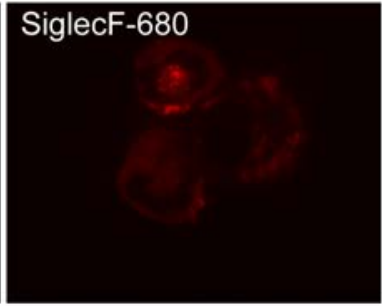

SiglecF-680

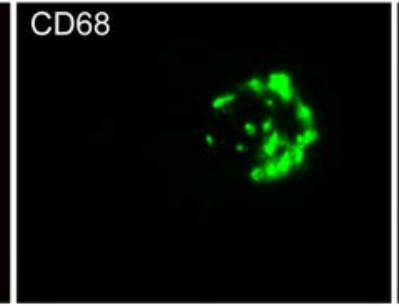

CD68
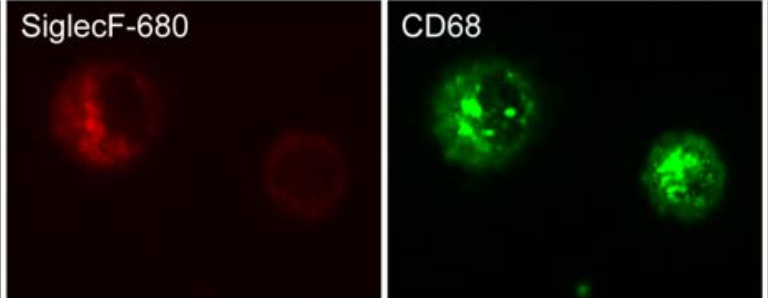

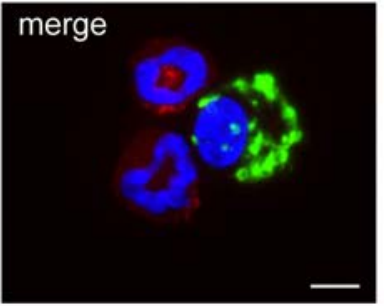

merge

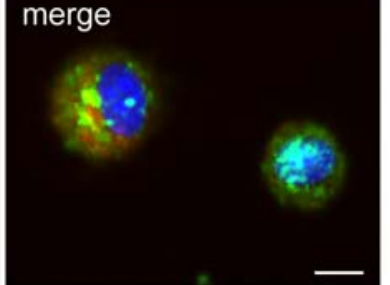

Figure 2. Expression pattern of Siglec-F. Immunohistochemistry and immunofluorescence Siglec- $F$ staining of lung sections and BAL cytospins of mice with EAAD ( $A-D$, upper panels) and controls ( $A-D$, lower panels). (A)- (B) represent sections of cryofrozen lungs stained with anti-Siglec- $F$ antibody. (C) Representative images of cytospins from BAL stained with anti-Siglec-F antibody and (D) of cytospins from BAL fluid co-stained with anti-SiglecF-680 and anti-CD68. In EAAD lungs, Siglec- $F$ is highly expressed in eosinophils surrounding the blood vessels $(b / v)$ and airways $(a / w)(A$, upper panel), while control lungs are almost free of Siglec-F staining (A, lower panel), indicating the lack of immune cell infiltration. Higher magnification of EAAD lung sections demonstrates Siglec-F staining on eosinophils (arrows, bilobed nucleus) and macrophages (arrow heads) (B, upper panel). In cytospins, eosinophils (bilobed nucleus) from EAAD animals ( $C$ and $D$ upper panel, arrows) demonstrate strong positive Siglec- $F$ staining, whereas macrophages from both, EAAD and control animals (C, arrow heads and D, positive CD68 staining) show a variety of Siglec- $F$ expression levels. Scale bars in A: $2.5 \mathrm{~mm}$; in B-D: $5 \mu \mathrm{m}$. doi:10.1371/journal.pone.0090017.g002

optical imaging. All intravenous (i.v.) injections and scanning procedures were performed under $2 \%$ isoflurane, $21 / \mathrm{min}$ oxygen anesthesia for a maximum time of $20 \mathrm{~min}$. The mice were sacrificed with an overdose of isoflurane after the last scan.

For treatment response studies, mice received either $25 \mu \mathrm{g}$ dexamethasone (Sigma-Aldrich, Hamburg, Germany) in $50 \mu \mathrm{l}$ PBS i.n. or $60 \mu \mathrm{g}$ beta-escin in $200 \mu \mathrm{l}$ PBS (Marinomed, Vienna, Austria) i.p. once a day from day 25 to 29. All i.n. procedures were performed under mild $2 \%$ isoflurane, $21 /$ min oxygen anesthesia of the mice.

\section{In vivo Optical Imaging}

To decrease autofluorescence, BALB/c mice were shaved and chemically depilated (Isana depilation crème, Rossmann) to remove the fur from thorax and abdomen. Optical imaging was performed by FRI using the Optix MX2 System (ART, Montreal, Canada), which comprises an interface for inhalation anesthetics and four pulsed lasers $(635,670,730$ and $785 \mathrm{~nm})$. During in vivo scans, mice were anaesthetized by inhalation with $2 \%$ isoflurane, 21/min oxygen for 15-20 min per scan. For detailed description of 


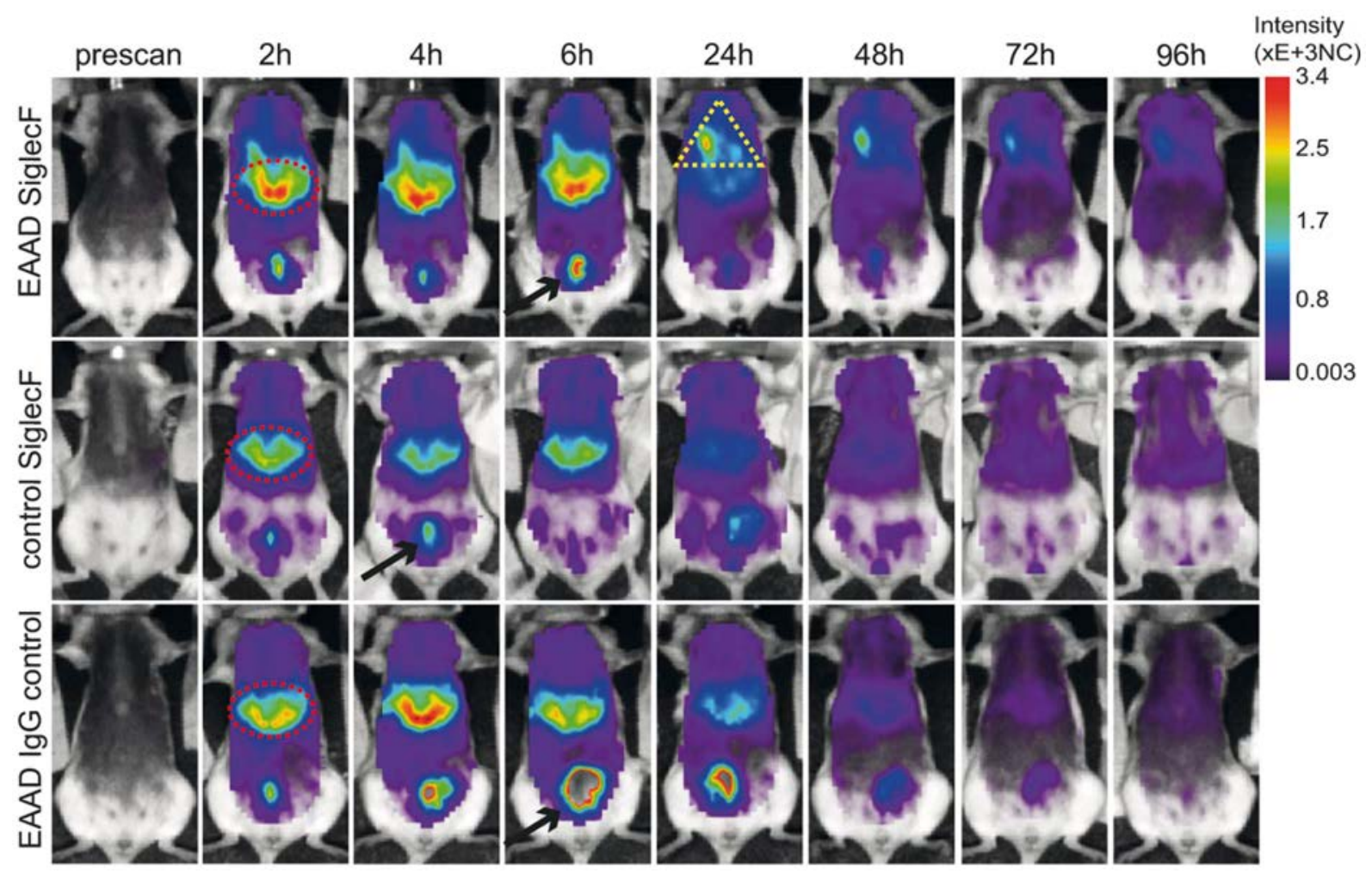

Figure 3. Time course of NIRF-labeled anti-Siglec-F distribution in the body. In vivo representative full body scans of EAAD (upper panel, $\mathrm{n}=8$ ) and control (middle panel, $\mathrm{n}=6$ ) mice injected with $12 \mu \mathrm{g}$ of anti-SiglecF-750, as well as EAAD mice injected with $12 \mu \mathrm{g}$ of Alexa 750-labeled anti-IgG2a isotype control antibody (lower panel, $n=5$ ) at the given time points. Fluorescence intensity distribution is displayed in normalized counts (NC). Excess anti-SiglecF-750 antibody accumulates within the liver (red elipse) and is excreted via the bladder (black arrows) within the first few hours after antibody administration. $24 \mathrm{~h}-48 \mathrm{~h}$ after anti-SiglecF-750 injection, EAAD mice, in contrast to all control animals, accumulate the Siglec-Fantibody in their lungs (yellow triangle). doi:10.1371/journal.pone.0090017.g003

the working principle of the Optix MX2 please refer to Dullin et al. [17].

OVA-challenged and control mice were prescanned to measure the autofluorescence signals of the animals. Three to four days post challenge, the animals were injected intravenously (i.v.) with either $12 \mu \mathrm{g}$ of anti-SiglecF-750 antibody $(\mathrm{n}=8$ for EAAD; $\mathrm{n}=6$ for controls), anti-SiglecF-680 antibody ( $\mathrm{n}=5$ for EAAD; $\mathrm{n}=4$ for controls) or 750-labeled rat IgG2a isotype control $(\mathrm{n}=5)$ in $150 \mu \mathrm{l}$ PBS and scanned at given time points. Alexa Fluor 750 fluorescence was measured using an excitation of $730 \mathrm{~nm}$ in combination with a $770 \mathrm{~nm}$ long-pass emission filter. Alexa Fluor 680 fluorescence was measured using an excitation of $670 \mathrm{~nm}$ in combination with a $700 \mathrm{~nm}$ long-pass emission filter. Scans were performed with a $1.5 \mathrm{~mm}$ (whole body scans) or $1.0 \mathrm{~mm}$ (lung scans) raster, a photon collection time (integration time) of $0.3-1 \mathrm{~s}$ per scan point and varying laser power. Intensity data and lifetime were analyzed with the OptiView-2-02-00 software (ART).

Fluorescence intensity data are displayed in normalized counts (NC), where the measured fluorescence intensity (counts) was normalized for varying laser power and integration times, allowing comparison of measurements with different settings. Data were quantified as average fluorescence intensity over a certain area of interest and subsequently corrected for autofluorescence by subtracting the average fluorescence intensity from the same region of interest in the respective prescans, as well as corrected for the dye to protein ratio of the different conjugates.

\section{Bronchoalveolar Lavage (BAL)}

Following imaging (72 h after antibody injection), mice were sacrificed with an overdose of isoflurane. BAL was performed by washing the airways gently three times with $500 \mu \mathrm{l}$ of $2 \%$ FCS/ PBS after exposing and cannulating the trachea. Volumes were pooled and then washed once in the same buffer. Recovered cells were counted in a haemocytometer and $3 \times 10^{4}$ cells were used for cytospins followed by Giemsa staining (Sigma Aldrich, Munich, Germany) for differential cell counting. Where indicated, cytospins were immunostained and counterstained with DAPI $(4 \mu \mathrm{g} / \mathrm{ml})$ for visualization of nuclei.

\section{Immunohistochemistry and Immunofluorescence}

Explanted lungs were cannulated and filled with $600 \mu \mathrm{l}$ of Tissue Tek OCT compound (Sakura Finetek Germany GmbH, Staufen, Germany) and immediately frozen in liquid nitrogen at $80^{\circ} \mathrm{C}$. Frozen lung sections of $5 \mu \mathrm{m}$ from untreated EAAD and control mice were cut on a Jung Frigocut 2800E cryostat microtome (Leica Microsystems, Wetzlar, Germany) and stained with monoclonal rat-anti-mouse-Siglec-F (BD Biosciences, Heidelberg, Germany) at $10 \mu \mathrm{g} / \mathrm{ml}$ in antibody-diluent (DAKO) and $4^{\circ} \mathrm{C}$, overnight (o.n.). Subsequently, sections were incubated with an anti-rat-biotinylated secondary antibody for $1 \mathrm{~h}$ at RT (BioLegend, Fell, Germany), followed by detection with avidinhorseradish-peroxidase (eBioscience, Frankfurt, Germany) for $1 \mathrm{~h}$ at RT. The sections were then counterstained with haematoxylin/ eosin (HE) and analysed by transmitted light microscopy with an Axioskop 2 microscope (Leica Microsystems, Wetzlar, Germany). 

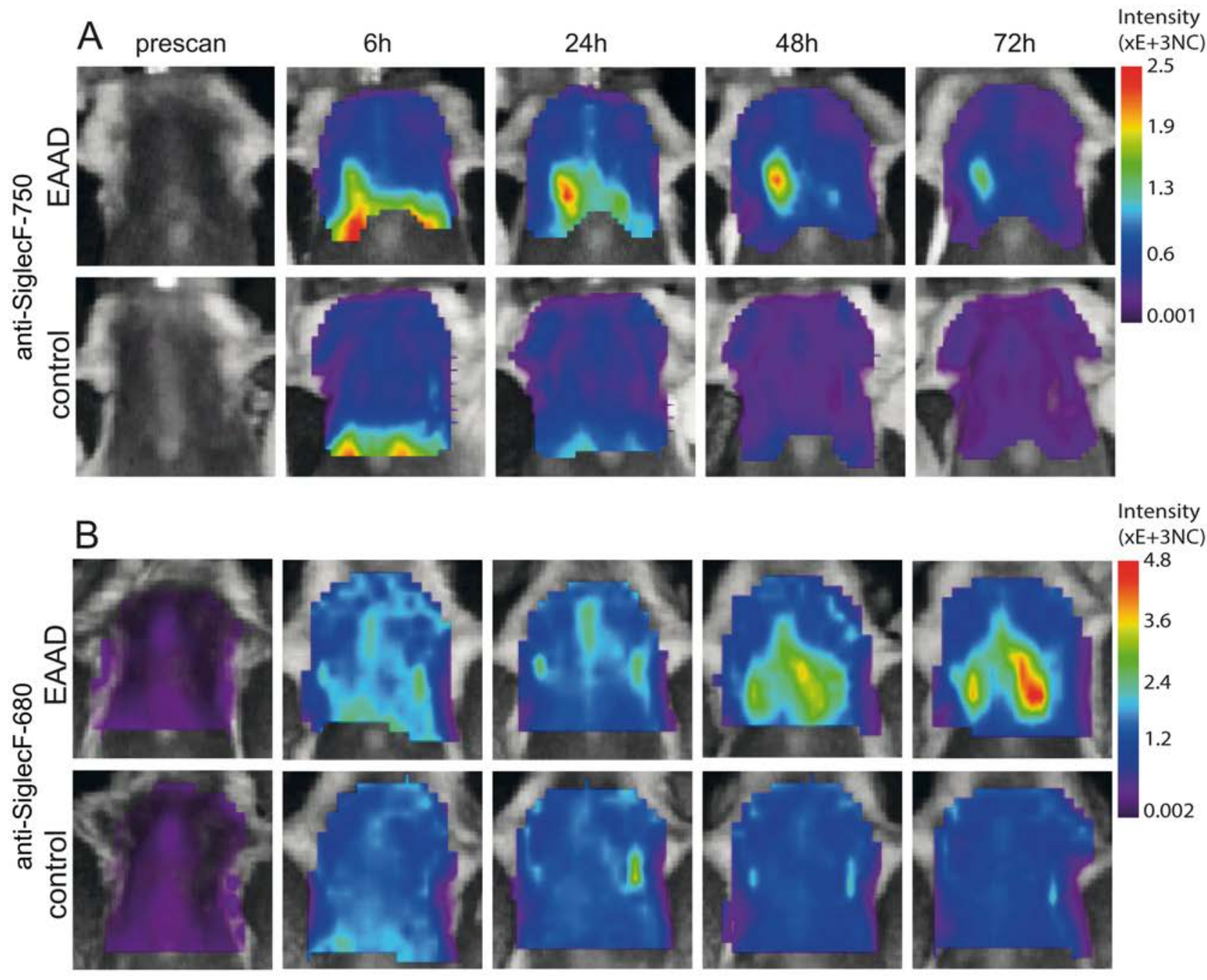

Intensity
$(x E+3 N C)$

C

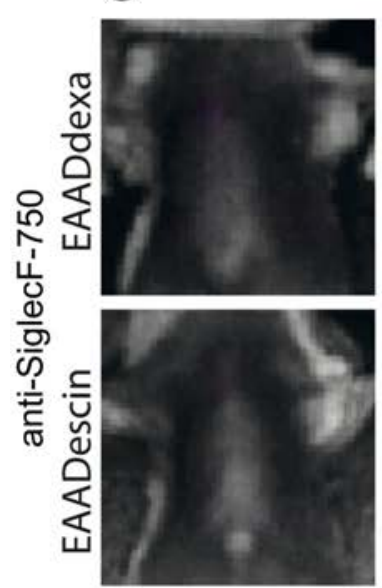

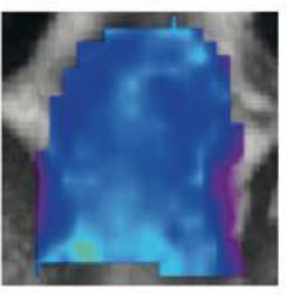
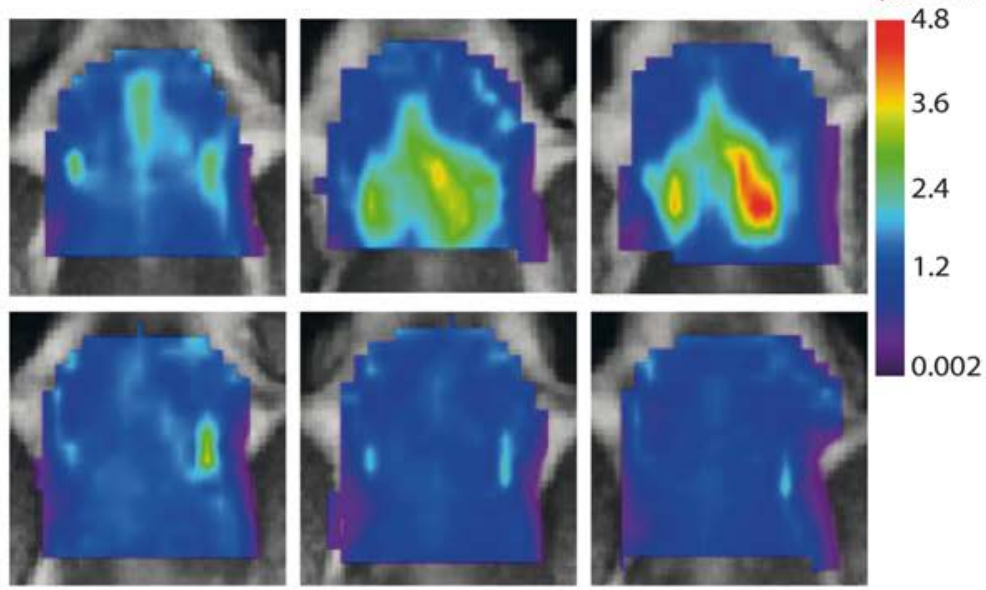

Intensity

$(x E+3 N C)$
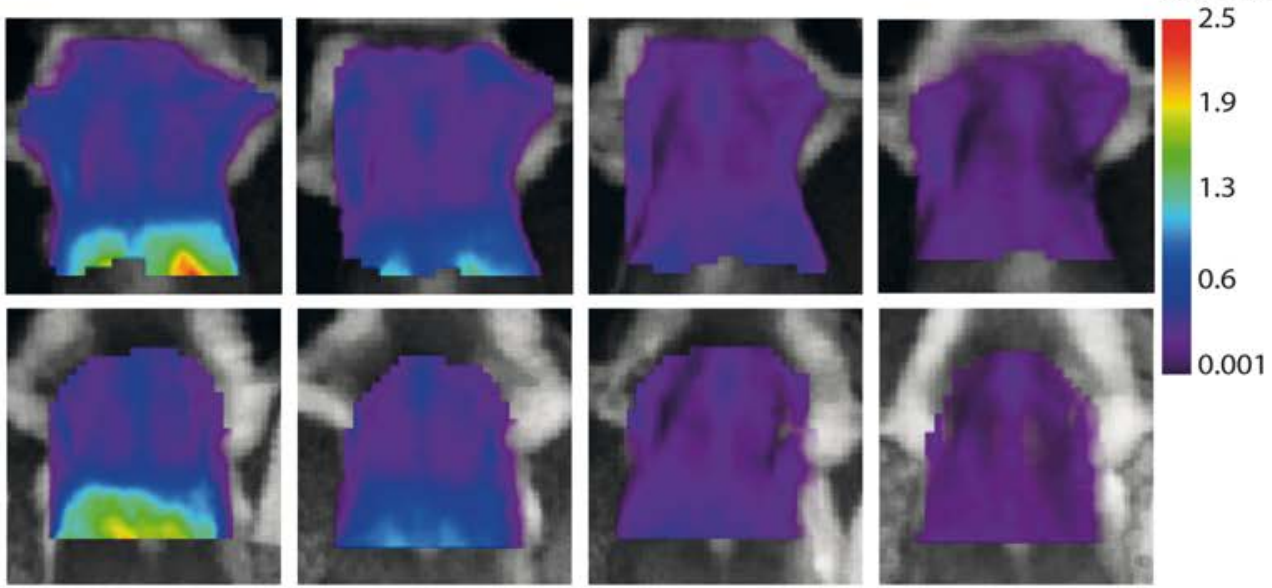

Figure 4. Time course of NIRFlabeled anti-Siglec-F distribution in the lung. In vivo lung scans of EAAD, control as well as dexamethasone and beta-escin treated animals before (prescan) and at $6 \mathrm{~h}, 24 \mathrm{~h}, 48 \mathrm{~h}$ and $72 \mathrm{~h}$ after antibody administration. Fluorescence intensity distribution is displayed in normalized counts (NC). In contrast to control mice (A, lower panel, $n=6)$, OVA-immunized mice have a marked accumulation of antiSiglecF-750 within the lungs from $24 \mathrm{~h}$, which decreases at $72 \mathrm{~h}(\mathrm{~A}$, upper panel, $\mathrm{n}=8$ ). Anti-SiglecF-680 also reveals significant differences between EAAD ( $B$, upper panel, $n=5$ ) and control $(B$, lower panel, $n=4)$ fluorescence intensities derived from the lung. EAAD mice treated with either dexamethasone ( $C$, upper panel, $n=5)$ or beta-escin ( $C$, lower panel, $n=5)$ have low intensities over the lung, similar to healthy control mice $(A$ and $B$, lower panels) at all scan times.

doi:10.1371/journal.pone.0090017.g004 

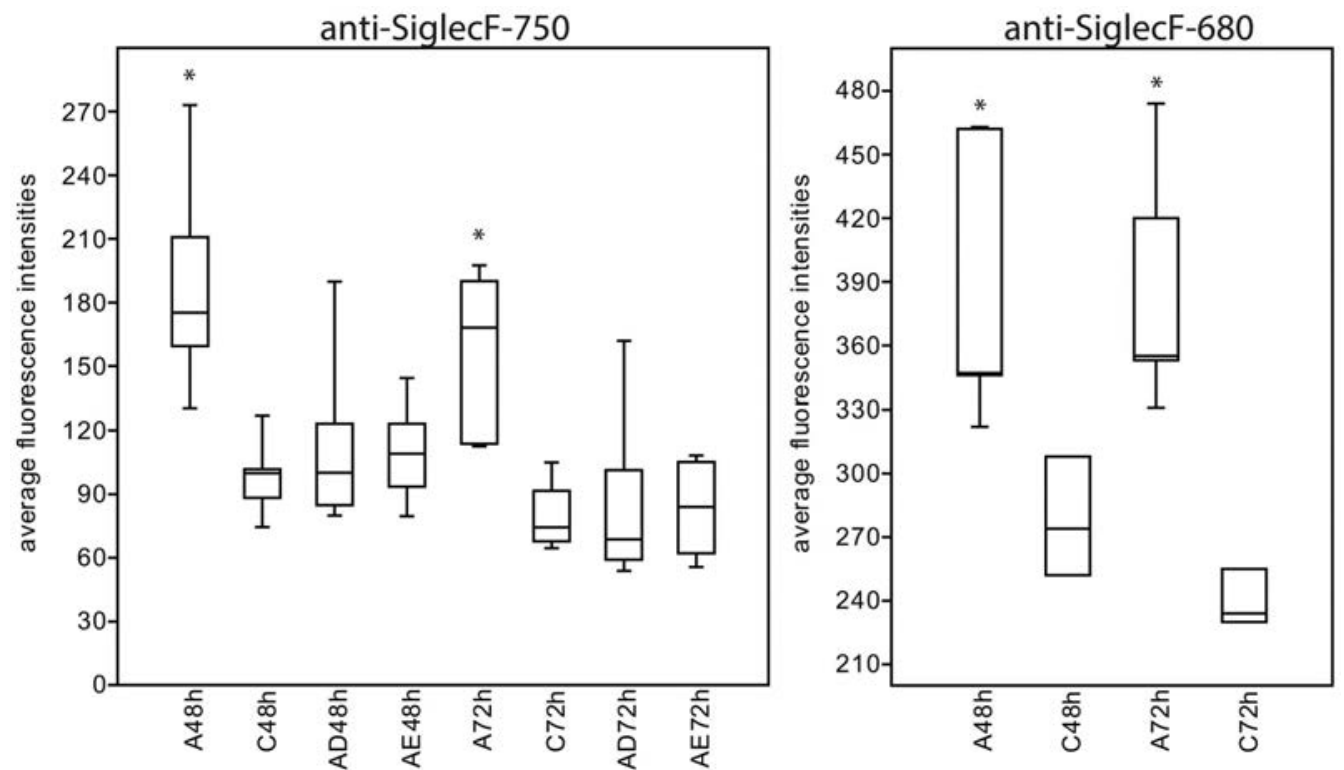

Figure 5. Quantification of in vivo imaging results. Box plot of average fluorescence intensities over the lung area for all groups at $48 \mathrm{~h}$ and $72 \mathrm{~h}$ after NIRF-labeled anti-Siglec-F antibody injection. Lung intensities of EAAD mice are significantly higher (represented by asterisk *) compared with control mice and treated mice at $48 \mathrm{~h}$ and $72 \mathrm{~h}$ after antibody application; $A=E A A D, C=$ control, $A D=E A A D$, dexamethasone treated, $\mathrm{AE}=\mathrm{EAAD}$, beta-escin treated.

doi:10.1371/journal.pone.0090017.g005

For NIRF microscopy, sections were stained with DAPI $(4 \mu \mathrm{g} /$ $\mathrm{ml}$ ) and anti-SiglecF-680. For fluorescence microscopy of lungs from anti-SiglecF-680 injected mice, cryosections were stained with anti-mouse-eosinophilic major basic protein (EMBP) antibody (clone S-16, Santa Cruz, Heidelberg, Germany) followed by anti-Alexa Fluor 488 secondary antibody, and/or anti-mouse CD68 antibody (clone FA-11, Abcam, Cambridge, UK), followed by anti-rat-Alexa Fluor 555 secondary antibody (Life Technologies $\mathrm{GmbH}$, Darmstadt, Germany). Images were acquired with a Leica CTR6000 fluorescence microscope equipped with a Leica DFC350FX camera.

\section{Statistical Analysis}

Statistical analysis was performed with Past [18] using a Welch t-test. P-values $<0.05$ were considered significant.

\section{Ethics Statement}

This study was carried out in strict accordance with the guidelines for the care and use of laboratory animals of the local ethics office of the University Medical Center Göttingen. This study was approved by the Committee on the Ethics of Animal Experiments of the Niedersächsisches Landesamt für Verbraucherschutz und Lebensmittelsicherheit (LAVES) (Permit Number:
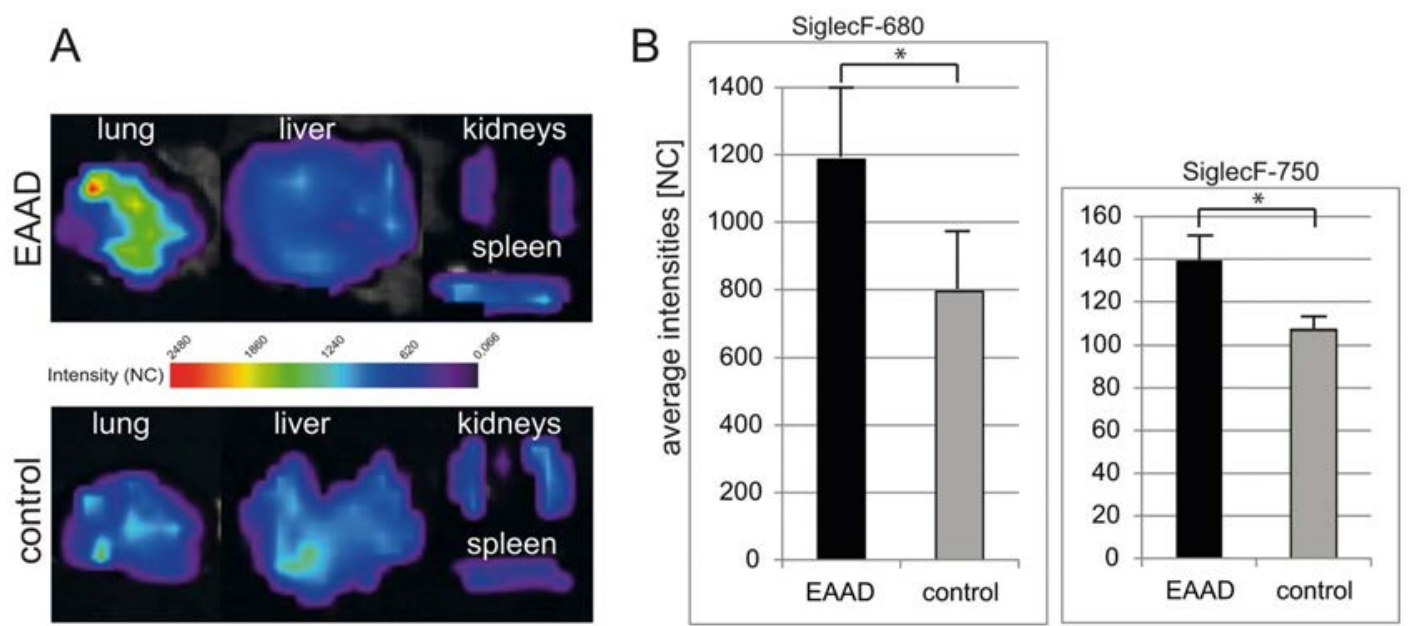

Figure 6. Ex vivo imaging results. (A) Representative images of fluorescence intensities of explanted lungs, livers, kidneys and spleens of EAAD (upper panel) and control mice (lower panel). (B) Bar graph of average fluorescence intensities of explanted lungs from mice injected with antiSiglecF-680 (left panel) or anti-SiglecF-750 (right panel). Ex vivo lung scans demonstrate a significant difference between signal intensities of EAAD lungs and healthy lungs (A and B), while liver, spleen and kidneys show low intensities in both EAAD and control mice $(A)$. NC = normalized counts. doi:10.1371/journal.pone.0090017.g006 

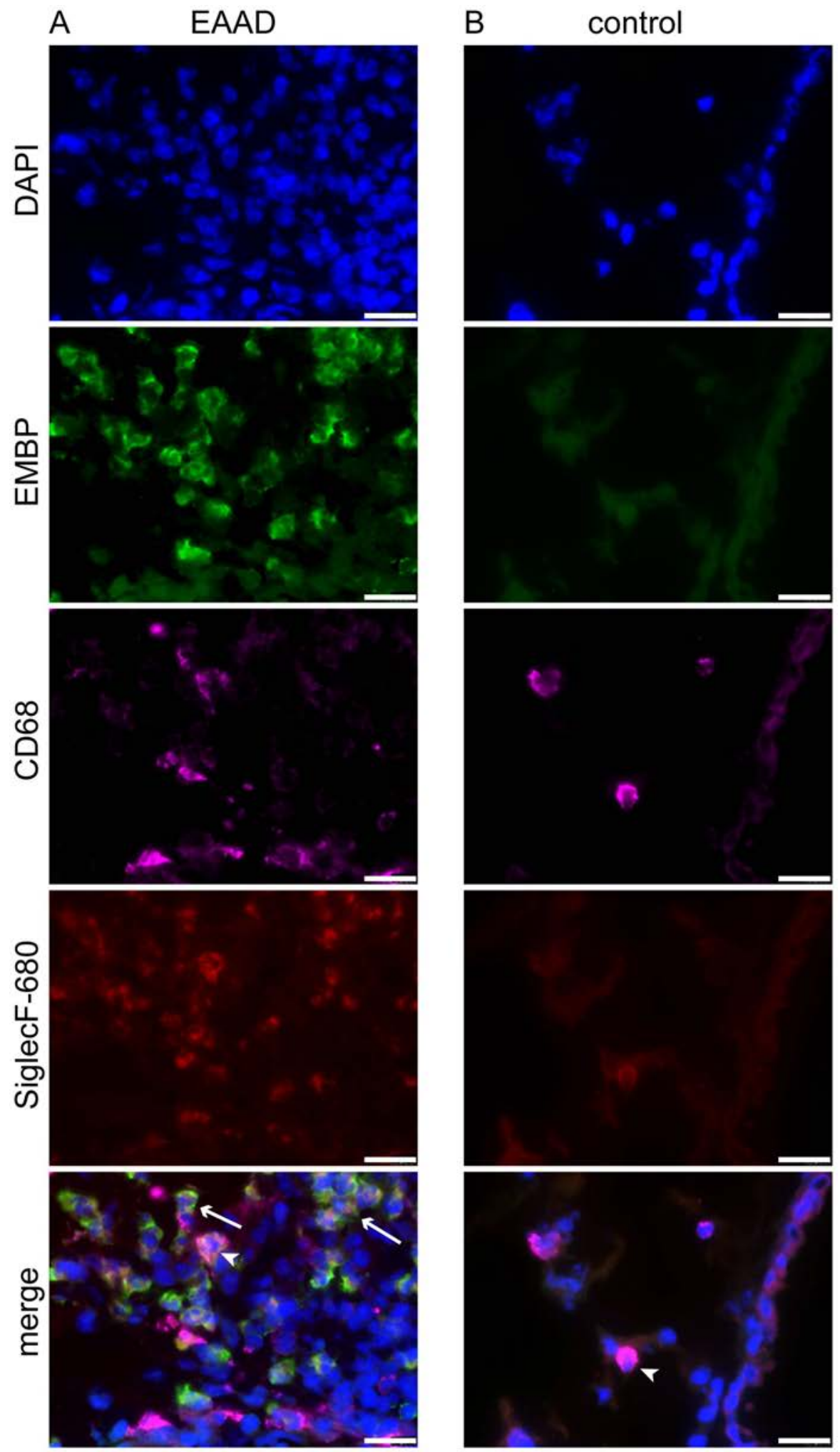

Figure 7. Anti-SiglecF-680 binds to eosinophils and macrophages. Fluorescence microscopy of cryosections from lungs of EAAD mice injected with anti-SiglecF-680 (A), confirms the binding of anti-SiglecF-680 (in green) to eosinophils (EMBP-positive, arrows in merge) and more weakly to macrophages, which were counterstained with anti-CD68 (magenta, arrow heads in merge). Lungs from healthy controls injected with antiSiglecF-680 (B) have a low number of Siglec-F positive cells, which are all CD68-positive and therefore most probably represent macrophages. Nuclei are stained blue with DAPI. Scale bars $=20 \mu \mathrm{m}$ doi:10.1371/journal.pone.0090017.g007 
33.9-42502-04-10/0134). All painful procedures were performed under anesthesia, and all efforts were made to minimize suffering.

\section{Results}

\section{Siglec-F is a Suitable Marker of Eosinophilia}

We first analysed the expression of Siglec-F on cells that accumulated in lungs of EAAD mice that did not receive the NIRF-labeled probe. Immunostaining of lung cryosections with an anti-Siglec-F antibody demonstrated a positive staining of cells that infiltrated the lung around blood vessels and airways of EAAD mice (Figure 2A, upper panel). However, only scattered cells within the lung tissue of control animals were Siglec-F positive (Figure 2A, lower panel). At higher magnification (100x, Figure 2B, upper panel), the positive cells in inflamed lungs are revealed to be mostly eosinophils as judged by their bilobed nuclei (arrows), and display a strong staining. A smaller number of weaker stained macrophages (large cells with unsegmented nuclei, arrow heads) were also detected in EAAD lung tissue. In control lung tissue the Siglec-F positive cells were all macrophages, as judged by their size and unsegmented nucleus (Figure 2B, lower panel, arrow head). As shown in Figure 2C, upper panel, immunostaining of cytospins from BAL fluid of EAAD mice with anti-Siglec-F antibody also revealed strong expression of Siglec-F on eosinophils, as confirmed by morphological appearance (cells with bilobed nuclei, arrows). Macrophages (larger cells, with unsegmented nuclei) revealed a variable degree of Siglec-F expression in both EAAD and control BAL (Figure 2C, arrow heads).

We then tested the two NIRF-labeled Siglec-F antibodies, antiSiglecF-750 and anti-SiglecF-680, to confirm that these antibodies too, detect eosinophils and macrophages. For this purpose, we performed immunostaining of cytospins from BALs of EAAD and control mice. To better distinguish between cell types, we counterstained the nuclei with DAPI and labeled with antiCD68, a common macrophage marker. We found that both NIRF-labeled antibodies were able to detect Siglec-F on eosinophils and macrophages. Figure 2D representatively shows the results for anti-SiglecF-680. In general, we found that macrophages with a high expression of CD68 also tended to exhibit a strong expression of Siglec-F.

\section{In vivo Detection of Siglec-F Expression}

To assess the suitability of anti-Siglec-F-antibody for in vivo detection of EAAD we first conjugated it to Alexa Flour 750, a near infrared fluorescence dye with an excitation maximum at $750 \mathrm{~nm}$. In this spectral range tissue autofluorescence is reduced due to minimal excitation of skin and hemoglobin, which are the main causes of autofluorescence. This approach improves signalto-background ratio and hence the limits of detection. We then injected $12 \mu \mathrm{g}$ of anti-SiglecF-750 i.v. into the tail veins of EAAD or control mice. As a negative control, EAAD mice were injected i.v. with an equal quantity of Alexa Fluor 750-labeled IgG2a isotype antibody at the same time point. Mice were scanned in vivo before (prescan) and after antibody injection for 4 days (24 h, $48 \mathrm{~h}, 72 \mathrm{~h}$ and $96 \mathrm{~h}$ ) to determine the distribution of the antibody in the whole body. Figure 3 shows representative images of full body scans of an EAAD anti-SiglecF-750 injected mouse (upper panel), a control anti-SiglecF-750 injected mouse (middle panel) and an EAAD mouse which received the isotype control antibody (lower panel) at the given time points. Within the first 6 hours after antibody administration, excess antibody accumulated within the liver (red ellipses in Figure 3) and was excreted via the bladder in all mice (black arrows in Figure 3). At $24 \mathrm{~h}$, most of the fluorescence signals over the liver and bladder area were cleared from all mice, which was similar in EAAD and healthy mice as well as in mice treated with the IgG2a control antibody. However, after $24 \mathrm{~h}$, EAAD mice accumulated anti-SiglecF-750 in their lungs (yellow triangle in Figure 3, upper panel). This signal was detectable over the following 2 days and decreased to background levels at $96 \mathrm{~h}$. In contrast, none of the control animals showed a specific signal within the lung at any time point (middle and lower panel).

To analyse the signals from the lungs in more detail, we performed in vivo lung scans by choosing a region of interest (ROI) over the lungs of the animals. These were performed with an increased raster resolution $(1 \mathrm{~mm})$ as well as higher integration time $(1 \mathrm{~s})$. To verify that the measured fluorescence intensities originated from the injected conjugates, we performed a lifetime analysis and compared the results with the lifetime of the pure conjugate. The lifetime of fluorescence signals measured in vivo over the lung region was the same as that of the pure conjugate measured ex-vivo (0.85 ns for anti-SiglecF-750 and $1.52 \mathrm{~ns}$ for antiSiglecF-680) and was substantially higher than the lifetime of the autofluorescence background measured in the prescan over the same region $(0.33 \mathrm{~ns}$ at $750 \mathrm{~nm}$ and $0.63 \mathrm{~ns}$ at $680 \mathrm{~nm}$ ) (data not shown).

Figure 4A shows representative images of lung scans of OVAinduced EAAD mice and healthy control mice before and $6 \mathrm{~h}$, $24 \mathrm{~h}, 48 \mathrm{~h}$ and $72 \mathrm{~h}$ after anti-SiglecF-750 application. During the first $6 \mathrm{~h}$, low fluorescence intensities were detected over the lungs in control and EAAD animals, which was probably, in part, due to the scattering of signals originating from the liver. This low signal decreased over the next 3 days in control mice (Figure 4A, lower panel). In contrast, OVA-immunized mice had a high fluorescence signal over the lung indicating an accumulation of NIRF-labeled antibody within the lungs over the following 3 days (Figure 4A, upper panel). Mice with EAAD exhibited a 1.4 (24 h scan, $\mathrm{p}=0,04)-2.0(48 \mathrm{~h}$ scan, $\mathrm{p}=0,00035$ and $72 \mathrm{~h} \mathrm{scan}, \mathrm{p}=0,00012)$ times higher anti-SiglecF-750 signal over the lungs compared to control animals (Figure 5, left panel). The presence of EAAD was confirmed by HE staining of lung cryosections at the end of the experiment (96 h after antibody administration), which demonstrated immune cell infiltration around the bronchi and vessels within the lung (data not shown).

To verify the results with a dye with a higher quantum yield, we used an anti-Siglec-F antibody coupled to Alexa Fluor 680 (antiSiglecF-680) in the same EAAD model and measured the signal intensities over the lung at the altered wavelength of $680 \mathrm{~nm}$ in EAAD and control animals. All other conditions and time points were unchanged. Figure 4B shows representative images of one animal illustrating that anti-SiglecF-680 also leads to high signal intensities in the lungs of EAAD mice (upper panel), while control animals displayed much lower signals (lower panel). Anti-SiglecF680 signal intensities were $1.4(48 \mathrm{~h}$ scan, $\mathrm{p}=0,01)-1.6(72 \mathrm{~h}$ scan, $p=0,0021)$ times higher in lungs of EAAD mice when compared to controls (Figure 5, right panel). Notably, the intensities with anti-SiglecF-680 were generally higher than those with anti-SiglecF-750, which was mainly due to differences in the quantum yields of the dyes $(0.12$ for Alexa Fluor 750 versus 0.36 for Alexa Fluor 680; www.lifetechnolgies.com), as the difference between the dye to protein ratios were already accounted for.

Peak average intensities of lung signals in animals with EAAD were observed across the range of detection time points, with 7 animals showing maximum intensity $24 \mathrm{~h}$ after antibody application, 1 exhibiting a maximum signal $48 \mathrm{~h}$, and 5 with maximum signal $72 \mathrm{~h}$ after anti-Siglec-F-750 or anti-SiglecF-680 injections. This is indicative of a difference in the progress of acute onset or resolution of EAAD with a varying accumulation of eosinophils 
and other infiltrating immune cells in the lungs. However, by $96 \mathrm{~h}$, all animals with acute EAAD had reduced specific fluorescence signals over the lungs.

Out of 13 OVA-immunized mice, only two displayed a homogenous fluorescence signal distribution over the lung, whereas all others showed an asymmetrical distribution of signal intensities (Figure 4A and 4B). Eight mice showed a higher signal on the right side of the lung, while 3 mice demonstrated more signal on the left side, reflecting differential cellular infiltration in different lung lobes, based on histological examination of lung tissue from EAAD mice (data not shown). In summary, fluorescence intensities over the lungs of EAAD mice in vivo were significantly higher compared to those of control mice at $48 \mathrm{~h}$ and $72 \mathrm{~h}$ after antibody application, as demonstrated in the box plot (Figure 5).

\section{Ex vivo Confirmation of Siglec-F Expression}

To verify that the in vivo measured signals originated from the lung, we performed scans of the excised lungs from animals sacrificed after the last in vivo scan, seen in Figure 6A. Ex vivo scans confirmed that lungs from EAAD mice displayed a significantly higher fluorescence signal intensity than control animals up to $96 \mathrm{~h}$ after i.v. injection of anti-SiglecF-750 (1.3 times higher in EAAD, $p=0,007)$ (Figure 6B, right panel) or anti-SiglecF-680 (1.5 times higher in EAAD, $\mathrm{p}=0,03$ ) (Figure 6B, left panel). Note, that here too, the average intensities with anti-SiglecF-680 are higher than with anti-SiglecF-750, due to the higher quantum yield of Alexa Fluor 680

Other organs such as liver, kidneys and spleen showed substantially lower Siglec-F signals in both EAAD and control animals (Figure 6A). Signals in liver and kidneys of control mice were generally somewhat higher than in the same organs of EAAD mice, which may be explained by a higher amount of unbound NIRF-labeled anti-Siglec-F antibody that is cleared by the liver and kidneys.

To analyze the binding of the injected anti-SiglecF-680 to lung cells in more detail, frozen tissue sections were visualized with a fluorescence microscope. To better distinguish between eosinophils and macrophages, lung sections were stained with an antibody directed against EMBP (eosinophilic major basic protein), an eosinophil marker and anti-CD68 antibody, a macrophage marker. Nuclei were visualized with DAPI. As seen in Figure 7A, anti-SiglecF-680 was mainly bound to eosinophils around bronchi and vessels in EAAD lungs (EMBP-positive, arrows in merged image), but was also present on some macrophages (CD68-positive, arrow head in merged image). Colocalization of anti-SiglecF-680 (red) and anti-EMBP (green) resulted in a yellow staining of eosinophils in the merged image. Healthy control lungs displayed few anti-SiglecF-680 positive cells (Figure 7B), which were all CD68 positive (arrow in merged image) and therefore, macrophages and demonstrated vesicle-like Siglec-F staining in the cytoplasm. These results confirm that the higher in vivo signals we found in EAAD mice derive mostly from antiSiglecF-NIRF antibody bound to eosinophils.

\section{In vivo Monitoring of EAAD in Response to Therapy}

To investigate the feasibility of anti-SiglecF-750 to assess the therapeutic response of mice with EAAD in vivo, we performed lung scans after treatment with dexamethasone or the natural compound beta-escin, as indicated in the Methods section in Figure 1. Both dexamethasone- and beta-escin-treated mice showed comparable signal intensities over the lungs that were similar to healthy control mice (Figure 4G) and significantly different to untreated EAAD animals at $48 \mathrm{~h}$ and $72 \mathrm{~h}$ after antibody application (Figure 5, left panel). Average lung intensities of dexamethasone-treated mice were $1.6(\mathrm{p}=0,01)$ and 1.8 $(p=0,0059)$ times lower than untreated EAAD lungs at $48 \mathrm{~h}$ and $72 \mathrm{~h}$, respectively. Average lung intensities of beta-escintreated mice were $1.8(\mathrm{p}=0,0034)$ and $2.0(\mathrm{p}=0,00079)$ times lower than in untreated EAAD mice at $48 \mathrm{~h}$ and $72 \mathrm{~h}$ after antibody injection. In dexamethasone-treated mice, the response to treatment, as evaluated by HE staining of lung cryosections from mice sacrificed $96 \mathrm{~h}$ after antibody administration, correlated with in vivo signal intensities. Mice that showed a low amount of cell infiltration and decreased bronchial wall thickness due to successful therapy, also had low fluorescence signals over the lung (Figure S1, C-D). Despite therapy with dexamethasone, 2 mice still had inflammation around bronchi and vessels and showed fluorescence signals over the lung above control levels (supplementary Figure 1, A-B). These results demonstrate that the reduction of lung cell infiltration upon treatment could be successfully monitored non-invasively by NIRF imaging with the anti-SiglecF-750 probe (Figure 4C, lower panel). In summary, both dexamethasone and beta-escin treatment reduced antiSiglec-F-750 lung signal intensity in EAAD mice to that of healthy controls.

\section{Discussion}

Here, we show that the antibody targeting Siglec-F is a novel NIRF probe that can be applied for non-invasive optical imaging of EAAD in mice, during the course of the disease as well as in response to therapy. We found that upon administration of antiSiglecF-750 and anti-SiglecF-680 probes there were significantly higher fluorescence signals in the lungs of mice with EAAD compared to healthy controls, demonstrating that NIRF imaging was successfully used to distinguish EAAD and healthy mice in vivo.

FRI-NIRF-based studies of the lung have, to date, found little use in preclinical in vivo studies. However, a recent study demonstrated the application of a novel NIRF-labeled probe containing dendritic polyglycerol sulfates to monitor inflammation in OVA-induced EAAD in mice, by targeting selectins [11]. Furthermore, the enzyme-based and commercially available activatable probes, MMPSense and ProSense (PerkinElmer) were shown to be useful for detection of lung inflammation $[9,10,19]$. These probes are optically silent in their inactivated state, but become fluorescent following activation by either matrix metalloproteinases (MMPs) or cathepsins. A concern is that these probes are not specific for lung inflammation, and might target inflammation elsewhere. Additionally, smart-probes activated by cathepsins and MMPs do not directly bind any target, leading to a high background from unbound probe. These factors do not apply for anti-Siglec-F antibody, as it binds directly to eosinophils. Since eosinophilia is one of the hallmarks of allergic asthma, Siglec-F represents a useful marker for disease imaging of EAAD.

In support of previously published studies $[13,20]$, we found a pronounced expression of Siglec-F on lung eosinophils of EAAD mice. Furthermore, the binding of the i.v. applied antibody to eosinophils was verified by NIRF microscopy on EAAD lung sections. We administered anti-Siglec-F at the peak of eosinophilia, which in this model is 3-4 days after the last i.n. challenge $[21,22]$, to achieve a high expression level of Siglec-F within the lung that could be detected by the NIRF-labeled antibody. Notably, several studies have shown that Siglec-F may play a role in the resolution of the acute allergic reaction by inducing eosinophil apoptosis $[13,20,23]$. For instance, in mice lacking Siglec-F, there is delayed resolution of lung eosinophilia and reduced peribronchial cell 
apoptosis in a model of EAAD [13]. In addition, the administration of an anti-Siglec-F antibody significantly reduced allergeninduced eosinophilic airway inflammation owing to the reduced production of eosinophils and an increase in apoptotic eosinophils in lung, blood, and bone marrow [13,20,23]. Based on these studies, anti-Siglec-F antibody has been considered as a therapeutic drug for eosinophilic disorders including acute and chronic asthma [20,24,25]. To avoid the potential anti-Siglec-F antibodyinduced eosinophil apoptosis effect on the course of disease, we administered the antibody 4 days after OVA challenge. However, we cannot rule out that the decline of the signal detected in EAAD mice within $24-48 \mathrm{~h}$ of receiving the antibody was caused by antiSiglec-F-induced apoptosis of eosinophils. We would argue that the decline of the fluorescence signal is mostly likely due to degradation or resorption of the antibody or a result of the normal physiological resolution of allergic inflammation. Nevertheless, it is tempting to speculate that anti-Siglec-F antibody could be used as a combined diagnostic and therapeutic tool for allergic eosinophilic inflammation for preclinical animal models.

Ex vivo results indicate that the anti-Siglec-F antibody not only targets eosinophils, but also binds to macrophages around the vessels and bronchioles of the EAAD lung tissue, which supports previous findings [14]. Because macrophages increase in number in the lungs of mice with EAAD, it is likely that the increased Siglec-F signal in EAAD mice as well as the low but measurable signal in control mice originated, in part, from the antibody binding to macrophages. Furthermore, macrophages are involved in the phagocytosis of apoptotic eosinophils during the resolution of eosinophilia [26,27], and probably the underlying mechanism for the observed Siglec-F expression in vesicle-like structures within macrophages from EAAD mice.

Even though anti-Siglec-F antibody does not bind exclusively to eosinophils and plays a role in eosinophil apoptosis, our results show that it can be used as a probe to distinguish allergic lung inflammation from healthy lung. There are additional eosinophil markers, such as CCR3, EMBP, CD23, CD48, and CD147, which are considered useful as potential in vivo targeting probes, but they also bind to other immune cells, such as mast cells or Th cells [28-31], and may affect the course of the disease to a larger degree than anti-Siglec-F.

Our choice to utilize NIRF imaging for these studies was based on several advantages over imaging methods such as MRI and PET. One advantage is that there is a low signal-to-noise ratio and high tissue penetration [32]. NIRF imaging using commercially available dyes, Alexa Fluor 680 and Alexa Fluor 750 [33] coupled to Siglec-F antibody, were highly suitable for distinguishing between EAAD and controls. The fluorescence signals we detected in vivo over the lung of EAAD mice were specific, as an isotype control antibody did not demonstrate fluorescence intensities above controls. Furthermore, the lifetime of these signals was the same as that of the pure probe and substantially higher than the lifetime of the autofluorescence background measured in prescans. We observed that Alexa Fluor 680, in general, exhibited higher fluorescence intensities, which was probably due to its higher quantum yield. However, this resulted in higher background levels and may have contributed to the lower signal ratios observed between EAAD and control mice injected with anti-SiglecF-680. The majority of EAAD mice showed a difference in signal intensity between the right and left lung, which reflects the distribution of inflammation in the lungs based on histological examination of lung tissue from EAAD mice. Our findings are supported by previous studies demonstrating heterogeneous distribution of allergic inflammation in the lung using CT and
MRI [34] and in a study of non-invasive optical tomography using NIRF-labeled smart probes [10].

Not only could normal, healthy lungs be distinguished from mice with allergic lung inflammation, but it was possible to monitor therapeutic efficacy with our anti-Siglec-F imaging approach. EAAD mice treated with glucocorticosteroids or betaescin exhibited reduced eosinophilic inflammation, as visualized by the NIRF-labeled anti-Siglec-F antibody. Treatment with dexamethasone and beta-escin reduces lung eosinophilia $[35,36]$ and in our experiments led to low intensity signals over the lungs of EAAD mice that were similar to healthy controls. These data confirm the suitability of anti-Siglec-F-NIRF as a method not only for detecting allergic lung inflammation but also for monitoring the efficacy of novel therapies for the treatment of EAAD in preclinical in vivo studies.

A feature that distinguishes EAAD from human asthma is the level of airway eosinophilia. The model used in these studies leads to approximately $30 \%$ eosinophils in the airways [22], which is higher than airway eosinophilia in humans. Nevertheless, this model is optimal for proof of concept for our imaging approach, because untreated EAAD mice have significantly higher lung eosinophilia compared with treated and healthy mice [37], which allows the differences between healthy, treated, and untreated groups to be easily distinguished. We would argue that our approach works well in small animals and could be used effectively in preclinical models. However, there are some limitations using this approach clinically. Recent studies demonstrate that Siglec-F functions differently in animals and humans. For example, Mao et al. showed, that Siglec-F-mediated apoptosis differed in magnitude and underlying mechanism in mice compared to Siglec-8-mediated human eosinophil apoptosis [38], which may be explained by the fact that Siglec-8 is a functional paraloq of Siglec$\mathrm{F}$, rather than a true ortholog. Moreover, other targets that were successfully used therapeutically in EAAD were ineffective in humans [30,39]. This indicates that the differences between EAAD and human asthma in regard to eosinophilia, T-cell response and AHR need to be considered [15] and entail optimization of both animal models and probes. Therefore, the use of anti-Siglec-F/Siglec-8 antibody as a theranostic tool for human asthma will require further study.

\section{Conclusion}

In conclusion, we demonstrate the suitability of NIRF-labeled anti-Siglec-F-antibody probe in combination with FRI imaging as a novel tool for non-invasive monitoring of allergic lung inflammation in mice, for monitoring treatment efficacy and progression of other eosinophil-related diseases.

\section{Supporting Information}

Figure S1 In vivo imaging results of treated mice correlate with peribronchial inflammation. The upper panel represents $48 \mathrm{~h}$ scans of 5 different EAAD mice treated with dexamethasone. Samples (A) and (B) demonstrate a low but measurable anti-SiglecF-750 signal in the lung (arrow heads) in comparison to $(\mathrm{C})-(\mathrm{E})$. The corresponding HE staining of lung cryosections at the end of the experiment (lower panel) shows the two samples with fluorescence signal have remaining cell infiltration (arrows) despite therapy. All other samples reveal a complete resolution of inflammation, as judged by the lack of infiltrating immune cells.

(TIF) 


\section{Acknowledgments}

We thank Sarah Greco, Roswitha Streich and Bettina Jeep for excellent help with the animal models and immunohistochemistry, and Stephen Kilfeather for fruitful discussions.

\section{References}

1. National Asthma Education and Prevention Program (2007) Expert Panel Report 3 (EPR-3): Guidelines for the Diagnosis and Management of AsthmaSummary Report 2007. J Allergy Clin Immunol 120: S94-138. doi:10.1016/ j.jaci.2007.09.043.

2. Al-Hajjaj MS (2011) Difficult-to-treat asthma, is it really difficult? Ann Thorac Med 6: 1-2. doi:10.4103/1817-1737.74268.

3. Kim HY, DeKruyff RH, Umetsu DT (2010) The many paths to asthma: phenotype shaped by innate and adaptive immunity. Nat Immunol 11: 577-584. doi:10.1038/ni. 1892 .

4. Bates JHT, Rincon M, Irvin CG (2009) Animal models of asthma. Am J Physiol Lung Cell Mol Physiol 297: L401-410. doi:10.1152/ajplung.00027.2009.

5. Blyth DI, Pedrick MS, Savage TJ, Hessel EM, Fattah D (1996) Lung inflammation and epithelial changes in a murine model of atopic asthma. Am J Respir Cell Mol Biol 14: 425-438.

6. Jannasch K, Missbach-Guentner J, Alves F (2009) Using in vivo imaging for asthma. Drug Discovery Today: Disease Models 6: 129-135. doi:10.1016/ j.ddmod.2009.12.003.

7. Napp J, Mathejczyk JE, Alves F (2011) Optical imaging in vivo with a focus on paediatric disease: technical progress, current preclinical and clinical applications and future perspectives. Pediatr Radiol 41: 161-175. doi:10.1007/s00247010-1907-0.

8. Weissleder R, Ntziachristos V (2003) Shedding light onto live molecular targets. Nat Med 9: 123-128. doi:10.1038/nm0103-123.

9. Cortez-Retamozo V, Swirski FK, Waterman P, Yuan H, Figueiredo JL, et al. (2008) Real-time assessment of inflammation and treatment response in a mouse model of allergic airway inflammation. J Clin Invest 118: 4058-4066. doi: $10.1172 / J C I 36335$.

10. Korideck H, Peterson JD (2009) Noninvasive quantitative tomography of the therapeutic response to dexamethasone in ovalbumin-induced murine asthma. J Pharmacol Exp Ther 329: 882-889. doi:10.1124/jpet.108.147579.

11. Biffi S, Dal Monego S, Dullin C, Garrovo G, Bosnjak B, et al. (2013) Dendritic Polyglycerolsulfate Near Infrared Fluorescent (NIRF) Dye Conjugate for NonInvasively Monitoring of Inflammation in an Allergic Asthma Mouse Model. PLoS ONE 8: e57150. doi:10.1371/journal.pone.0057150.

12. Bochner BS (2009) Siglec-8 on human eosinophils and mast cells, and Siglec-F on murine eosinophils, are functionally related inhibitory receptors. Clin Exp Allergy 39: 317-324. doi:10.1111/j.1365-2222.2008.03173.x.

13. Zhang M, Angata T, Cho JY, Miller M, Broide DH, et al. (2007) Defining the in vivo function of Siglec-F, a CD33-related Siglec expressed on mouse eosinophils. Blood 109: 4280-4287. doi:10.1182/blood-2006-08-039255.

14. Feng Y, Mao H (2012) Expression and preliminary functional analysis of SiglecF on mouse macrophages. J Zhejiang Univ Sci B 13: 386-394. doi:10.1631/ jzus.B1 100218 .

15. Finkelman FD, Wills-Karp M (2008) Usefulness and optimization of mouse models of allergic airway disease. Journal of Allergy and Clinical Immunology 121: 603-606. doi:10.1016/j.jaci.2008.01.008.

16. Dyer KD, Garcia-Crespo KE, Killoran KE, Rosenberg HF (2011) Antigen profiles for the quantitative assessment of eosinophils in mouse tissues by flow cytometry. J Immunol Methods 369: 91-97. doi:10.1016/j.jim.2011.04.009.

17. Dullin C, Zientkowska M, Napp J, Missbach-Guentner J, Krell H-W, et al. (2009) Semiautomatic landmark-based two-dimensional-three-dimensional image fusion in living mice: correlation of near-infrared fluorescence imaging of Cy5.5-labeled antibodies with flat-panel volume computed tomography. Mol Imaging 8: 2-14.

18. Hammer Ø, Harper DAT (n.d.) i Ryan, PD (2001) PAST: Paleontological statistics software package for education and data analysis. Palaeontologia Electronica 4: 9 .

19. Haller J, Hyde D, Deliolanis N, de Kleine R, Niedre M, et al. (2008) Visualization of pulmonary inflammation using noninvasive fluorescence molecular imaging. J Appl Physiol 104: 795-802. doi:10.1152/japplphysiol.00959.2007.

\section{Author Contributions}

Conceived and designed the experiments: MAM CD EPG MME FA. Performed the experiments: MAM CD MM. Analyzed the data: MAM CD. Contributed reagents/materials/analysis tools: MAM CD EPG MME FA. Wrote the paper: MAM MME FA.

20. Song DJ, Cho JY, Lee SY, Miller M, Rosenthal P, et al. (2009) Anti-Siglec-F Antibody Reduces Allergen-Induced Eosinophilic Inflammation and Airway Remodeling. J Immunol 183: 5333-5341. doi:10.4049/jimmunol.0801421.

21. Ohkawara Y, Lei XF, Stämpfli MR, Marshall JS, Xing Z, et al. (1997) Cytokine and eosinophil responses in the lung, peripheral blood, and bone marrow compartments in a murine model of allergen-induced airways inflammation. Am J Respir Cell Mol Biol 16: 510-520.

22. Mojtabavi N, Dekan G, Stingl G, Epstein MM (2002) Long-lived Th2 memory in experimental allergic asthma. J Immunol 169: 4788-4796.

23. Zimmermann N, McBride ML, Yamada Y, Hudson SA, Jones C, et al. (2008) Siglec-F antibody administration to mice selectively reduces blood and tissue eosinophils. Allergy 63: 1156-1163. doi:10.1111/j.1398-9995.2008.01709.x.

24. Farid SS, Mirshafiey A, Razavi A (2012) Siglec-8 and Siglec-F, the new therapeutic targets in asthma. Immunopharmacol Immunotoxicol 34: 721-726. doi:10.3109/08923973.2011.589453.

25. Rubinstein E, Cho JY, Rosenthal P, Chao J, Miller M, et al. (2011) Siglec-F inhibition reduces esophageal eosinophilia and angiogenesis in a mouse model of eosinophilic esophagitis. J Pediatr Gastroenterol Nutr 53: 409-416. doi:10.1097/ MPG.0b013e3182182ff8.

26. Pappas K, Papaioannou AI, Kostikas K, Tzanakis N (2013) The role of macrophages in obstructive airways disease: chronic obstructive pulmonary disease and asthma. Cytokine 64: 613-625. doi:10.1016/j.cyto.2013.09.010.

27. Uller L, Persson CGA, Erjefält JS (2006) Resolution of airway disease: removal of inflammatory cells through apoptosis, egression or both? Trends Pharmacol Sci 27: 461-466. doi:10.1016/j.tips.2006.07.006.

28. Ochi H, Hirani WM, Yuan Q Friend DS, Austen KF, et al. (1999) T helper cell type 2 cytokine-mediated comitogenic responses and CCR3 expression during differentiation of human mast cells in vitro. J Exp Med 190: 267-280.

29. Sallusto F, Mackay CR, Lanzavecchia A (1997) Selective expression of the eotaxin receptor CCR3 by human T helper 2 cells. Science 277: 2005-2007.

30. Wegmann M (2011) Targeting Eosinophil Biology in Asthma Therapy. American Journal of Respiratory Cell and Molecular Biology 45: 667-674. doi: 10.1165/rcmb.2011-0013TR.

31. Ackerman SJ, Kephart GM, Habermann TM, Greipp PR, Gleich GJ (1983) Localization of eosinophil granule major basic protein in human basophils. J Exp Med 158: 946-961. doi:10.1084/jem.158.3.946.

32. Hilderbrand SA, Weissleder R (2010) Near-infrared fluorescence: application to in vivo molecular imaging. Curr Opin Chem Biol 14: 71-79. doi:10.1016/ j.cbpa.2009.09.029.

33. Berlier JE, Rothe A, Buller G, Bradford J, Gray DR, et al. (2003) Quantitative Comparison of Long-wavelength Alexa Fluor Dyes to Cy Dyes: Fluorescence of the Dyes and Their Bioconjugates. J Histochem Cytochem 51: 1699-1712. doi: $10.1177 / 002215540305101214$

34. Aysola R, de Lange EE, Castro M, Altes TA (2010) Demonstration of the heterogeneous distribution of asthma in the lungs using CT and hyperpolarized helium-3 MRI. J Magn Reson Imaging 32: 1379-1387. doi:10.1002/ jmri.22388.

35. Druilhe A, Létuvé S, Pretolani M (2003) Glucocorticoid-induced apoptosis in human eosinophils: mechanisms of action. Apoptosis 8: 481-495.

36. Lindner I, Meier C, Url A, Unger H, Grassauer A, et al. (2010) Beta-escin has potent anti-allergic efficacy and reduces allergic airway inflammation. BMC Immunol 11: 24. doi:10.1186/1471-2172-11-24.

37. Bosnjak B, Tilp C, Tomsic C, Dekan G, Pieper MP, et al. (2014) Tiotropium bromide inhibits relapsing allergic asthma in BALB/c mice. Pulm Pharmacol Ther 27: 44-51. doi:10.1016/j.pupt.2013.09.004.

38. Mao H, Kano G, Hudson SA, Brummet M, Zimmermann N, et al. (2013) Mechanisms of Siglec-F-induced eosinophil apoptosis: a role for caspases but not for SHP-1, Src kinases, NADPH oxidase or reactive oxygen. PLoS ONE 8: e68143. doi:10.1371/journal.pone.0068143.

39. Fulkerson PC, Rothenberg ME (2013) Targeting Eosinophils in Allergy, Inflammation and Beyond. Nat Rev Drug Discov 12. Available: http://www. ncbi.nlm.nih.gov/pmc/articles/PMC3822762/. Accessed 13 January 2014. 


\title{
Tracking of inhaled near-infrared fluorescent nanoparticles in lungs of SKH-1 mice with allergic airway inflammation
}

\author{
M. Andrea Markus ${ }^{1, \S}$, Joanna Napp ${ }^{1,2,3, \S}$, Thomas Behnke ${ }^{4}$, Miso Mitkovski ${ }^{5}$, Christian Dullin ${ }^{3}$, Stephen Kilfeather ${ }^{6}$, \\ Ute Resch-Genger ${ }^{4}$, Frauke Alves ${ }^{1,2,3}$ \\ ${ }^{1}$ Department of Haematology and Medical Oncology, University Medical Center Göttingen, Germany \\ 2 Department of Molecular Biology of Neuronal Signals, Max-Planck-Institute for Experimental Medicine, \\ Göttingen, Germany \\ ${ }^{3}$ Department of Diagnostic and Interventional Radiology, University Medical Center Göttingen, Germany \\ ${ }^{4}$ Biophotonics Division, BAM Federal Institute for Materials Research and Testing, Berlin, Germany \\ ${ }^{5}$ Light Microscopy Facility, Max-Planck-Institute for Experimental Medicine, Göttingen, Germany \\ ${ }^{6}$ Aeirtec Limited, Newcastle upon Tyne, United Kingdom \\ $\S$ These authors made equal contributions. \\ Corresponding author: Frauke Alves; Department of Haematology and Medical Oncology, University Medical \\ Center Göttingen, Robert-Koch Str. 40, 37075 Göttingen, Germany; falves@gwdg.de
}

\begin{abstract}
Molecular imaging of inflammatory lung diseases such as asthma has been limited to date. In asthma, the recruitment of innate immune cells to the airways is central to the inflammation process. This study exploits these cells for imaging purposes within the lung, using inhaled polystyrene nanoparticles loaded with the near infrared fluorescence (NIRF) dye Itrybe (Itrybe-NPs). By means of in vivo and ex vivo fluorescence reflectance imaging (FRI) of an ovalbumin (OVA)-based allergic airway inflammation (AAI) model in hairless SKH-1 mice, we show that subsequent to intranasal application of Itrybe-NPs, AAI lungs display significantly higher fluorescence intensities than lungs of control mice for at least $24 \mathrm{~h}$. Ex vivo immunofluorescence analysis of lung tissue demonstrates the uptake of Itrybe-NPs predominantly by $\mathrm{CD} 68^{+} \mathrm{CD} 11 \mathrm{c}^{+} \mathrm{ECF}-\mathrm{L}^{+} \mathrm{MHCII}{ }^{\text {low }}$ cells, identifying them as alveolar M2 macrophages (AMs) in the peribronchial and alveolar areas. The in vivo results were validated by confocal microscopy and overlapping tile analysis, showing a significantly larger amount of Itrybe-NP-containing AMs in lungs of AAI mice than in controls. Itrybe-NPs were not found in lung draining lymph nodes, indicating that antigenpresenting cells other than AMs migrate to lymph nodes for interaction with T-cells in this model. This simple and elegant imaging approach may advance monitoring of asthma in vivo over time and aid the investigation of the role that AMs play during lung inflammation. Furthermore, it allows for tracking of inhaled nanoparticles and can hence be utilized for studies of the fate of potential new nanotherapeutics.
\end{abstract}

KEYWORDS: Itrybe nanoparticles, in vivo near infrared fluorescence (NIRF) imaging, experimental asthma, allergic airway inflammation mouse model, cell tracking, alveolar macrophages.

Nanoparticles (NPs) are of increasing interest in preclinical and clinical research and diagnosis. Because they are capable of conjunction with different cell types they provide a tool for cell tracking and have been particularly explored in the field of stem cell therapy and cancer to understand cell migratory pathways and the fate of grafted cells. Furthermore, the rapid development of nanotechnology has generated a plethora of nanomaterials as candidates for novel delivery systems for target-specific therapeutic drugs as well as for the diagnosis of diseases and assessment of treatment efficacy. The chemical properties and the preparation of NPs are well established and optimized with respect to resulting particle sizes, small size distributions and various surface chemistries and also with respect to the preparation of fluorophore-encoded particles. ${ }^{1-4}$ However, there is still a need for their biological profiling and examination in vivo. Several recent studies of the interaction of such nanoparticles with biological systems have demonstrated the large influence of the particle surface functionalization (chemical nature and number of surface groups) and charge on such interactions including the formation of a protein corona and cellular uptake. However, no general statements can be made at present because previous research has used a range of different model systems and sometimes poorly analytically 
characterized particles and has applied different methods to assess possible toxicity due to the lack of standardized measurements. ${ }^{5-10}$ Hence, it is often necessary to study the influence of the respective tailor made particle systems on the specific biological system of interest.

Moreover, with the advent of nanotherapeutics in clinical practice, ${ }^{11}$ and the increasing manufacture and use of products based on nanotechnology ${ }^{12}$, there is an ever increasing need to investigate the fate of NPs in the body and to determine in which cell types they accumulate. In addition, the stability of NPs in the cellular microenvironment, as well as their trafficking and retention within different cells and organs require examination. ${ }^{13,14}$ Furthermore, established international regulatory authority governance of use of foreign substances in diagnostics and therapeutics dictates the safety thresholds for incorporation of foreign substances into healthcare. NPs are foreign substances and if administered for diagnostic or therapeutic purposes will need to undergo rigorous toxicological examination before incorporation into healthcare.

Tissue macrophages derive from monocytes and greatly increase in numbers in inflammation that commonly occurs in diseased tissue. Hence, this cell is clearly an attractive target for new nanotherapeutics as well as for diagnostic purposes. The uptake of NPs by macrophages has been repeatedly shown in different biological systems and during disease. In particular, imaging of tumor-associated macrophages has been explored for the purpose of prognostic information, definition of tumor margins and measuring therapeutic response. $^{15-17}$

Along with magnetic NPs, fluorescent NPs have been abundantly used for imaging macrophages, in both in vitro and in vivo assays. ${ }^{18,19}$ Above all, targeted, optically active contrast agents have improved the diagnosis and therapeutic assessment in oncology. ${ }^{20}$ We successfully applied polystyrene NPs (PSNPs) loaded with the bright NIR-emissive asymmetric cyanine Itrybe for tumor detection in vitro and in a mouse tumor model. ${ }^{21}$ PSNPs are especially attractive for in vivo imaging as polystyrene is generally considered as inert and non-toxic. ${ }^{22,23}$ PSNP preparation via miniemulsion polymerization is well established and these particles are commercially available in a large range of different sizes with a narrow size distribution and various surface chemistries. $^{24,25}$ Moreover, control experiments performed with accordingly prepared dye-doped particles in a dispersion of 5\% BSA/PBS buffer revealed the absence of dye leakage. ${ }^{26}$
Here, we decided to use Itrybe-NPs to track macrophages in a mouse model of acute allergic inflammation (AAI) because the number of alveolar macrophages (AM) is significantly increased in asthma. ${ }^{27}$ Allergic asthma is a chronic inflammatory disease of the lungs characterized by a variable degree of reversible bronchoconstriction, airway hyperresponsiveness (AHR) and increased mucus production, features that have been reproduced in mice by sensitization with different allergens such as ovalbumin (OVA). ${ }^{28}$ Allergen challenge induces a strong immune response that leads to profound immune cell infiltration of the lung airways, activation of AMs and thickening of the airway epithelium with a marked goblet cell hyperplasia, all of which can be found in both asthmatic humans and airway immunized mice. ${ }^{29}$ AMs are part of the innate immune system and one of the first cell types that allergens encounter. In a healthy lung, they form $95 \%$ of the cell load in bronchoalveolar lavage (BAL), the remainder being mainly lymphocytes. Particles inhaled into the lower airways are rapidly phagocytized by these cells and their number increases significantly during AAI. ${ }^{30}$ Controversy exists about the role AMs play during the development or progress of asthma. Recent evidence suggests that AMs are associated with both antiinflammatory and pro-inflammatory functions. ${ }^{27}$ Moreover, it is still unclear whether AMs play a part in inducing immune tolerance by antigen-presenting capabilities or if this function is largely attributed to dendritic cells. $^{31,32}$

To date, non-invasive preclinical animal studies of lung disease have mainly facilitated the anatomical or structural assessment of the diseased lung and/or require the use of radioactive agents or X-rays (PET, SPECT, CT). ${ }^{33}$ Optical imaging has emerged as an important alternative, as it represents a rapid, inexpensive and relatively simple technique that enables the detection of specific targets and functional events in a live animal over time ${ }^{34}$, in particular when combined with near infrared fluorescent (NIRF) probes. Such probes have revealed several advantages over other fluorescent dyes as NIR light can penetrate deeper into the tissue and measurements in this spectral region benefit from comparably lower tissue autofluorescence. ${ }^{35}$ Moreover, they lack radioactivity and do not create toxic waste. Hence, NIRF imaging is considered as a real future alternative to nuclear imaging, the gold standard of functional imaging.

In this study we show that fluorescence reflectance imaging (FRI) in combination with bright NIRemissive non-toxic Itrybe-NPs is an ideal technique to specifically monitor the presence of AAI in an experimental allergic inflammation SKH-1 mouse 
model. We demonstrate that these NPs are specifically taken up by AMs in the lung and show, non-invasively, that AAI mice display significantly higher fluorescence intensities over the lung than controls. In addition, we find that such NPs provide an excellent tool to track lung macrophages and further substantiate the AMs as the most efficient phagocytes. We believe this imaging approach could be applied to pursue the fate of inhaled fluorescent NPs in the future, thereby assessing their suitability in theranostic nanomedicine.

\section{Results}

\section{SKH-1 mice are susceptible to AAI induction}

Because the fur of mouse strains used in asthma research to date, such as $\mathrm{BALB} / \mathrm{c}$ or $\mathrm{C} 57 \mathrm{BL} / 6$, interferes with fluorescence imaging by absorbing and scattering of light and requires removal of the hair before imaging, we have considered the hairless but immunocompetent SKH-1 mouse strain a worthy alternative. ${ }^{36}$ To the best of our knowledge, SKH-1 mice have not previously been used in asthma research. Induction of AAI in SKH-1 mice using a conventional OVA model (Figure 1A) resulted in characteristic features of allergic inflammation, comparable to that in BALB/c mice, as demonstrated by lung histology, BAL cell count and cytokine expression. H\&E staining of lung paraffin sections revealed typical infiltration of immune cells around the bronchi and blood vessels of the lung (Figure 1B). Periodic acid - Schiffs (PAS) staining of lung paraffin sections showed goblet cell hyperplasia and excess mucus production (Figure 1C). Eosinophil numbers in BALs of AAI mice was found to be on average $60 \pm 1.6$ $\%$ of the total cell count in OVA-induced immunized mice compared to $0.4 \pm 0.38 \%$ in control mice (Figure 1D). OVA-specific IgE and IgG1 were significantly elevated in sera of AAI mice (Figure 1E), as were mRNA expression levels of cytokines IL-4, IL-5, IL10 and IL-13 in AAI lung tissues (Figure 1F). These results demonstrate that OVA-sensitization and challenge lead to a robust eosinophilic inflammation and Th2 response in SKH-1 mice. Macrophage count in BALs of AAI mice was well correlated with eosinophil numbers (Supplementary Figure 1) with a Pearson Correlation Coefficient $r$ of 0,58 .

\section{Characterization of Itrybe-NPS}

Itrybe-NPs are 100 nm-sized carboxy-modified polystyrene nanoparticles loaded with the NIRF dye Itrybe, an asymmetric cyanine, via an established staining procedure. ${ }^{37}$ In this procedure, a dyecontaining tetrahydrofuran (THF) solution $(0.5 \mathrm{mM})$ was added to an aqueous particle suspension $(0.5 \mathrm{w} \%)$.
The addition of THF leads to swelling of the polymer matrix. The hydrophobic fluorophore molecules can then diffuse into the matrix and adsorb on the apolar polymer chains. Thus, no functional groups or chemical reaction is necessary for dye labeling. Subsequently, the NPs are thoroughly washed with ethanol to ensure complete removal of all unincorporated fluorophores from the particle surface and the swelling solvent is exchanged for water to induce the contraction of the swollen polymer particles. This leads to the physical and sterical entrapment of the dye molecules inside the NPs.

The material and spectroscopic properties as well as the in vitro characteristics of Itrybe-NPs are summarized in Figure 2. Characterizing of the size distribution of colloidally stable aqueous dispersions of Itrybe-NPs via nanoparticle tracking analysis (NTA) revealed a mean hydrodynamic diameter $\mathrm{d}_{\mathrm{H}}$ of $108+/$ $8 \mathrm{~nm}$ and high monodispersity (Figure 2A). Atomic force microscopy (AFM) showed the spherical shape and non-porous surface of the NPs (Figure 2B). ItrybeNPs show broad, unstructured and almost environment-insensitive absorption $(500 \mathrm{~nm}-700 \mathrm{~nm})$ and emission $(700 \mathrm{~nm}-900 \mathrm{~nm})$ bands with an absorption maximum at $605 \mathrm{~nm}$ and an emission maximum at $760 \mathrm{~nm}$, respectively. The broad absorption band provides a great versatility in the choice of the excitation wavelength, rarely found in NIRF dyes, and the large Stokes Shift of about 3400 $\mathrm{cm}^{-1}$ is ideal for the separation of scattered excitation light from fluorescence (Figure 2C). To test if ItrybeNPs are taken up by AMs in vitro, we incubated 100 $\mathrm{ng} / \mathrm{ml}$ NPs with $5 \times 10^{5} \mathrm{MH}-\mathrm{S}$ cells, an immortalized and adherent mouse alveolar macrophage cell line, over $5 \mathrm{~h}$ (data not shown) and $24 \mathrm{~h}$ (Figure 2D and E). The MH-S cells readily take up Itrybe-NPs within 5 hours of incubation. As can be seen in the representative image in Figure 2D, a strong active uptake of the particles is observed after 24h of incubation at $37^{\circ} \mathrm{C}$, which was completely inhibited, when the cells were maintained at $4^{\circ} \mathrm{C}$.

\section{In vivo monitoring of AAI by means of Itrybe-NPS}

To assess the suitability of Itrybe-NPs for monitoring the presence of AAI, we intranasally (i.n.) applied 100 $\mathrm{nm}$ NPs in OVA-induced AAI or control mice. Mice were scanned in vivo by FRI $1 \mathrm{~h}, 5 \mathrm{~h}$ and $24 \mathrm{~h}$ after NP application to investigate the distribution of Itrybe-NPs in whole bodies and in the lung over time. Full body scans revealed that fluorescence signals above background are detectable over the lung area as early as $1 \mathrm{~h}$ after NP application. Some animals (AAI mice and controls) also showed measurable fluorescence intensities in the stomach and intestine as well as in the 
bladder (data not shown). To analyze the fluorescence intensities derived from the lungs in more detail, we performed in vivo scans by choosing a region of interest (ROI) outlining the lungs of the animals. These were performed with an increased raster resolution (1 $\mathrm{mm}$ ) as well as a higher integration time (1 s). To verify that the measured fluorescence intensities originated from the injected Itrybe-NP, we performed a lifetime (LT) analysis and compared the results with the LT of the Itrybe-NPs in vitro in solution (Supplementary Figure 2). The fluorescence LT measured in vivo over the lung region was $0.8 \mathrm{~ns}$ as measured in vitro, which is substantially longer than the LT of the autofluorescence background measured in the prescan in the same region $(0.4-0.5 \mathrm{~ns})$. This indicates that the fluorescence intensities measured over the lung are specific for Itrybe.

Figure 3A shows representative images of lung scans of OVA-induced AAI mice and healthy control mice before and at $1 \mathrm{~h}, 5 \mathrm{~h}$, and $24 \mathrm{~h}$ post i.n. NP application. We found a significantly higher accumulation of Itrybe-NPs in lungs of OVAimmunized mice than in control mice at every time point (Figure 3C). Peak average intensities over the lung were nearly identical at $1 \mathrm{~h}$ and $5 \mathrm{~h}$ (153.8 and 154.2) post NP application. Some animals showed higher fluorescence intensities in the lung at $1 \mathrm{~h}$ or 24 $\mathrm{h}$ after i.n. NP application, indicative of an interindividual variability in the progress of acute onset of AAI with a varying accumulation of immune cells in the lungs of mice. AAI mice exhibited 2.9, 1.7 and 1.4 times higher NP-derived signals (average intensity over the lung minus average intensity of the prescan) in the lungs at $1 \mathrm{~h}, 5 \mathrm{~h}$ and $24 \mathrm{~h}$ respectively, when compared to control animals.

To confirm that the intensities measured in vivo originated from the lung, we performed FRI scans of the excised lungs from animals sacrificed $24 \mathrm{~h}$ after i.n. NP application. Ex vivo scans confirmed our in vivo data by displaying about 3.5 fold higher fluorescence intensities in AAI lungs than in control lungs (Figure $3 \mathrm{~B}$ and $\mathrm{D})$.

\section{Itrybe-NPs are phagocytized by bronchial and alveolar macrophages}

To analyze the location of the Itrybe-NP derived signals on a cellular level and thereby identify the cells that phagocytize the NPs in vivo, cytospins from BALs as well as frozen lung tissue sections were immunofluorescently stained with a fluorophorelabeled antibody against the macrophage marker CD68. Cytospins of BALs from lungs explanted $24 \mathrm{~h}$ after NP application demonstrated that Itrybe-NPs were taken up by CD68-positive $\left(\mathrm{CD}^{+} 8^{+}\right)$cells in both AAI and control mice (Figure 4A) but not by any other cells, such as eosinophils, which are easily distinguished by their fragmented or donut shaped nucleus (Figure 4A, left panel).

Immunofluorescence microscopy of cryosections from entire lungs explanted $24 \mathrm{~h}$ after NP instillation also showed Itrybe-NPs in $\mathrm{CD}^{+} 8^{+}$immune cells in both AAI and control tissue (Figure 4B). As shown in Figure 4B, Itrybe-NP loaded macrophages were predominantly found in alveolar spaces and in the bronchial lumen. In lungs from AAI mice, we never found NPs in macrophages located within inducible bronchus-associated lymphoid tissue (iBALT) - an ectopic lymphoid tissue formed in the lung after pulmonary infection or inflammation. However, Itrybe NP-filled macrophages often clustered around iBALT (Figure 4B), suggesting that Itrybe-NP-filled macrophages are not able to migrate into, but may be recruited by iBALT.

The total number of CD68- and Itrybe-NP-positive cells was obtained from merged, overlapping z-stacks, acquired with a confocal microscope and covering a representative area of $5.0 \times 2.8 \times 0.032 \mathrm{~mm}^{3}$ (Figure $5 \mathrm{~A})$. Specificity of the detected Itrybe-NP signals was insured by measuring the emission spectrum in several Itrybe-NP containing ROIs and comparing it with the emission spectra of ROIs devoid of Itrybe-NPs and with the emission spectrum of the pure Itrybe-NP solution (Supplementary Figures 3 and 4). The emission maximum of Itrybe-NPs on tissue slices (located at about $710 \mathrm{~nm}$ ) measured with the confocal microscope appears to be blue shifted compared to that measured in vitro $(760 \mathrm{~nm})$ with a calibrated spectrofluorometer (Figure 2C), which has been corrected for the wavelength-dependent spectral responsivity of the instrument. ${ }^{38}$ This apparent spectral deviation is ascribed to the wavelength-dependent spectral responsivity of the confocal microscope originating from the detector and the transmission of other optical components in the detection channel, which distorts the measured spectrum on tissue slices in an instrument-specific manner and accounts for the apparent blue shift in emission.

One tiled image of each 6 AAI and 6 control lung cryosections was analyzed. In AAI lungs we detected 4 times as many Itrybe-NP-containing AMs than in controls (Figure 5B). This difference clearly accounts for the increased fluorescence intensities detected by in vivo imaging in AAI lungs. As seen in Figure 5C, the number of Itrybe-NPs - containing cells form 2 clusters that correspond to AAI (red) and control (blue) mice, based on the fact that controls contain a lower total 
number of $\mathrm{CD} 68^{+} \mathrm{AMs}$ when compared to AAI mice and thus have a lower amount of Itrybe-NP containing $\mathrm{CD}^{+} 8^{+}$cells. Stitched images of lung sections also revealed that in AAI lungs Itrybe-NPs accumulate more often in clusters around bronchi and blood vessels when compared to control lungs, where they were more evenly spread (Figure 5A).

Although AMs in lung cryosections from AAI mice often appeared larger and consequently filled with more NPs when compared to controls, we could not find any significant difference in NP-load by quantification of mean fluorescence intensities in CD68/NP-colocalized cells (data not shown).

Because cross reactivity of anti-CD68 antibodies has been reported with fibroblasts and activated endothelial cells, ${ }^{39}$ and in order to exclude uptake of Itrybe-NPs by dendritic cells, we double-stained lung cryosections with anti-CD68 and anti-CD11c antibodies. CD11c is mainly expressed on dendritic cells (DCs), whereas most, if not all, AMs express low or intermediate amounts of CD11c. ${ }^{40}$ As DCs do not express CD68 ${ }^{41}$ and interstitial macrophages, fibroblasts and endothelial cells are all CD11cnegative, ${ }^{42}$ we considered a positive $\mathrm{CD} 68^{+} \mathrm{CD} 11 \mathrm{c}^{+}$ double staining a reliable method for identifying the cells as AMs, a detection method that was used by others before. ${ }^{43}$

As seen in Figure 6A, Itrybe-NPs were only found in $\mathrm{CD} 68^{+} \mathrm{CD} 11 \mathrm{c}^{+}$cells and were therefore considered to be phagocytized by AMs only. Co-staining with antiCD68 and anti-MHCII antibodies (Figure 6B) demonstrated that the Itrybe-NPs were only seen in $\mathrm{CD}^{+} 8^{+} \mathrm{MHCII}^{\text {low }}$ cells. This clearly confirms that cells that have taken up Itrybe-NPs are not dendritic cells, which express high levels of MHCII. A low expression of MHCII on Itrybe-NPs positive cells also suggests that Itrybe-NPs are preferentially taken up by alternatively activated (M2) macrophages rather than by classically activated macrophages (M1), which tend to express higher MHCII. This was further confirmed by double-staining of lung cryosections with antiCD68 and an antibody against ECF-L, a lectin primarily expressed on M2 macrophages. Figure $6 \mathrm{C}$ representatively shows that all Itrybe-NPs visualized by fluorescence microscopy were found in $\mathrm{CD} 68^{+} \mathrm{ECF}$ $\mathrm{L}^{+}$cells, suggesting that NPs are taken up by alternatively activated M2 macrophages. In summary, the immunofluorescence staining illustrates that ItrybeNPs were phagocytized by $\mathrm{CD} 8^{+} \mathrm{CD} 11 \mathrm{c}^{+} \mathrm{MHCII}{ }^{\text {low }} \mathrm{ECF}^{-\mathrm{L}^{+}}$cells, representing bronchial and alveolar M2 macrophages.
While we did not detect any Itrybe-NPs in $\mathrm{CD}^{-} 8^{-} \mathrm{MHC}^{\text {high }}$ cells in any of the sections examined, an indication that dendritic cells do not take up these NPs, we cannot completely rule out the occasional uptake of Itrybe-NPs by dendritic cells.

The possible uptake of Itrybe-NPs by lung epithelial cells was also examined by immunofluorescence studies with antibodies directed against the transmembrane protein podoplanin, a heavily $\mathrm{O}-$ glycosylated protein, present on alveolar epithelial type I cells (AT1) as well as prosurfactant protein C (proSPC), a marker for alveolar type II cells (AT2). ${ }^{44}$ While we never found any Itrybe-NPs in proSP-C ${ }^{+}$AT2 cells, we observed the location of some Itrybe-NPs in the cytoplasm of podoplanin ${ }^{+}$AT1 cells (Figure 7).

To assess if the pattern of Itrybe-NP uptake by AMs is different at an earlier or later time point, we performed anti-CD68 immunostaining with cryosections of lungs explanted $5 \mathrm{~h}, 48 \mathrm{~h}$ or $72 \mathrm{~h}$ after i.n. Itrybe-NP instillation. Similar to the $24 \mathrm{~h}$ time point, we found Itrybe-NPs in $\mathrm{CD} 68^{+} \mathrm{AMs}$, in both AAI and control lungs. In contrast to findings obtained $24 \mathrm{~h}$ after Itrybe-NP instillation, at $5 \mathrm{~h}$ many free Itrybe-NPs were still seen in alveoli and bronchioles, representing NPs which were not or not yet phagocytized by AMs (Figure 8). At $48 \mathrm{~h}$ and $72 \mathrm{~h}$ after Itrybe-NP application, the pattern of NP-distribution was comparable to that obtained after $24 \mathrm{~h}$ (Figure 8), with most Itrybe-NPs phagocytized by $\mathrm{CD} 8^{+} \mathrm{CD} 11 \mathrm{c}^{+} \mathrm{MHCII}^{\text {low }} \mathrm{ECF}^{-\mathrm{L}^{+}}$alveolar $\mathrm{M} 2$ macrophages.

Controversy exists about the pro-inflammatory role of macrophages, with conflicting results regarding the capability of antigen-primed AMs to migrate to lung draining lymph nodes and activate T-cells. ${ }^{45} \mathrm{We}$ therefore tested whether NP-containing AMs can be found in lung draining lymph nodes $24 \mathrm{~h}, 48 \mathrm{~h}$ or $72 \mathrm{~h}$ post NP application. However, we could not detect any Itrybe-specific signals at any time point analyzed in either AAI or control lymph node cryosections (data not shown), suggesting that the NP-loaded macrophages observed are either not able to, or are not required to, migrate to lung draining lymph nodes under allergic airway conditions.

\section{Discussion}

To our knowledge this is the first application of NIRFloaded NPs for the in vivo detection of inflammatory response by FRI in a conventional OVA-induced allergic airway inflammation mouse model (AAI). We could demonstrate that uncomplicated i.n. application of non-toxic Itrybe-NPs was sufficient to distinguish between AAI and control mice, showing that the 
measured fluorescence intensities over the lung area were significantly higher than those from controls.

Molecular imaging of lung diseases and in particular AAI using fluorescence imaging has been very limited up to now. Three in vivo approaches have been reported with NIRF labeled probes so far where higher fluorescence intensities were observed in AAI versus controls for OVA-induced allergic asthma models: one targeting eosinophilia by using a NIRF-labeled antiSiglecF antibody ${ }^{46}$ and the others monitoring the increase of typical inflammation markers such as matrix metalloproteinases ${ }^{47}$ or P-Selectins. ${ }^{48}$ All of these approaches used NIRF-labeled probes, as NIRF poses a number of advantages for FRI, such as high signal-to-background ratio and high tissue penetration. Our own approach also exploits these advantages of NIRF by utilizing NPs loaded with the NIRF dye Itrybe. In addition, we established AAI in hairless and immunocompetent SKH-1 mice and demonstrated that these mice respond equally well to the induction of allergic inflammation as commonly used mouse strains, such as BALB/c, by developing a pronounced eosinophilia, goblet cell hyperplasia, mucus production and $\mathrm{iBALT}$ in their lungs. These mice also displayed typical features of a Th2 allergic response such as elevated OVA-specific IgE and IgG1 serum levels as well as significantly higher IL-4, IL-5, IL-10 and IL-13 cytokine expression than controls. The use of hairless SKH-1 mice allows a further improvement of fluorescence detection owing to the reduction of light scattering and absorbance due to the lack of fur.

In addition, our intranasal application of the NP probe, presents the most direct route to lung tissue. Intravenously applied NPs tend to be rapidly cleared by the liver or kidneys, depending on the size, or accumulate in the spleen and lymph nodes, as these organs contain a high number of macrophages. ${ }^{49}$ While some animals displayed fluorescence signals in the stomach due to swallowing of the probe, we did not detect any NP-derived signals in the liver, kidney, spleen or lymph nodes. This underlines the high potential of intranasally applied Itrybe-NPs for noninvasively distinguishing inflamed from healthy lungs. Hence, this NP-based approach in general, and our Itrybe-NPs specifically, present a promising tool for the in vivo monitoring of lung pathologies where macrophages are involved as well as for the preclinical validation of new therapeutics in asthma mouse models.

In our imaging strategy, we found that macrophages readily capture and retain our NIRF-probe in the lung, as they represent one of the innate immune cells which significantly increase in numbers upon airway inflammation. NPs have been used to image macrophages before, for example using iron oxide or gadolinium particles in combination with MRI. In this way, inflammatory processes and the related macrophage infiltration were detected in atherosclerosis, myocardial infarction, stroke, multiple sclerosis, rheumatoid arthritis and kidney transplantation in small animal models. ${ }^{50}$ Nanomaterial-based contrast agents for computer tomography (CT) have also been used for tracking macrophages. However, X-rays require relatively high concentrations of NPs to delineate individual cells. ${ }^{51}$ Even NP-loaded macrophages themselves have been used as contrast agents. For example Madsen et al (2013) used MRI to detect the migration of NP-loaded exogenous macrophages into the brain. ${ }^{52}$ In a previous study we tracked intratracheally applied barium sulfate-loaded macrophages in the lung of mice with AAI by using synchrotron radiation inline free propagation phase contrast $\mathrm{CT}^{53}$ Imaging of endogenous alveolar macrophages has to date only been shown with gold-NPs ${ }^{54}$ and superparamagnetic iron oxide NPs (SPIOs). ${ }^{55}$ To the best of our knowledge our study is the first to use fluorescently labeled NPs to track pulmonary macrophages. Because macrophages increase in number during the process of AAI and are the main phagocytic cell in the lung, we explored the source of the increased in vivo signal which we detected in lungs of AAI mice by undertaking an ex vivo characterization of the uptake of Itrybe NPs. As we demonstrate, Itrybe NPs were readily taken up by AMs, but not by DCs, interstitial macrophages or any other innate cell. In accordance with the study by Geiser et al ${ }^{54}$, Itrybe NPs are taken up by macrophages of both diseased and healthy animals and no discriminating mechanism of phagocytosis was apparent with the microscopy methods we used. We identified NP-containing cells as $\mathrm{CD} 68^{+} \mathrm{CD} 11 \mathrm{c}^{+} \mathrm{ECF}-\mathrm{L}^{+} \mathrm{MHCII}{ }^{\text {low }}$ representing alveolar M2 macrophages. The role of the two major types of AMs, M1 (classically activated) and M2 (alternatively activated) macrophages, in human allergic asthma as well as mouse AAI, are complex and controversial. It has been proposed that M1 are Th1-driven, while M2 cells are induced by and can drive $\mathrm{Th} 2$ responses, but increasing evidence suggests the parallel development and involvement of both these cell types in asthma. ${ }^{27}$ It seems that while M1 macrophages may be beneficial to prevent allergic sensitization, in already established asthma they may promote the development of M2 macrophages. ${ }^{56} \mathrm{M} 2$ macrophages are associated with asthma, but it is still unclear if they actively contribute to asthma pathogenesis. ${ }^{57}$ In our study, we detected NPs only in M2 cells, regardless of disease status. We therefore assume that the uptake of NPs by M2 cells is not related or dependent on the presence of asthma, in 
accordance with the fact that these cells are involved in phagocytosis of foreign pathogens and apoptotic cells. It remains to be examined whether there is a difference in the amount of Itrybe-NPs taken up by AAI macrophages when compared to controls or whether AAI M2 macrophages process NPs differently to those of controls. A systematic analysis of the uptake and processing of Itrybe-NPs in relation to disease status and the effect NPs may have on the exacerbation of AAI is in preparation and will provide important information about the role AMs play in the relationship between AAI and inhaled NPs.

We were not able to detect any Itrybe-NPs in lung draining lymph nodes and therefore found no evidence of macrophage trafficking to lymph nodes. The latter finding is consistent with AMs being efficient phagocytes but poor antigen-presenting cells, whereas DCs are poor phagocytes but the most competent cell population for $\mathrm{T}$-cell activation. ${ }^{43,58}$ Alternatively, it may indicate that, when activated as M2 type, their antigen presenting capacity would be confined to their locality in respiratory tissue in conditions relating to the airway allergic model used. We further found that NP-filled AMs often clustered around iBALT. This could indicate that cells accumulated in BBALT, in particular B-cells, positively influence the activation and recruitment of macrophages, a mechanism that has recently been suggested for B-cells in iBALT of cigarette smoke - induced COPD. ${ }^{59}$ We also detected small amounts of Itrybe-NPs in AT1 cells, the structural cells of the lung which are responsible for gas exchange. These findings confirm former in vitro and in vivo studies that epithelial cells are capable of taking up NPs ${ }^{60,61}$ and should be considered in the future design of NP-based drug delivery systems.

Our tiling experiments confirmed the in vivo results by demonstrating a higher number of Itrybe-NPcontaining AMs in AAI than in controls. It also enabled a semi-quantitative calculation of the fluorescence intensity in AAI as compared to controls, which may be used as an indication of the percentage of NPs that can reach the different parts of the lung. Moreover, our results revealed that Itrybe-NPcontaining AMs cluster around bronchi and blood vessels in AAI, whereas there was a more homogenous distribution in controls. Future experiments may involve spectrally resolved, 3-dimensional, overlapping, confocal scans of the entire lung tissue sections, which would allow for absolute measurements of amount of NP present, as well as their localization within specific cell organelles or lung area.
As NPs are increasingly used as targeted drug delivery systems, ${ }^{62}$ their characterization and rigorous toxicological examination is of growing importance for clinical application. This includes establishing their fate and within this, the kinetics of the uptake, distribution and dispersal. Transport via macrophages is clearly involved in the latter and uptake of NPs by macrophages has been recently exploited for novel nanotherapeutics. ${ }^{63}$ Identification of location in tissues and cells then assists in the direction of toxicology focusing on the potential toxic effects on tissues and cell compartments of accumulation. Macrophages can traffic from the lungs to other organs and examination of their capacity to deliver NPs between sites is therefore of value in assessing NP kinetics. Nanoparticles may not be inert passengers within macrophages, but may have significant effects on their function and even influence their longevity. Evidence of such influence would be important for the interpretation of findings obtained from research of biological systems in which NPs are used as research tools. In this regard, Itrybe-NPs in combination with in vivo optical imaging and high resolution fluorescence microscopy represent a powerful tool to trace macrophages in the entire lung and provide new information of the fate of inhaled NPs. In addition, the biological stability which is provided by the encapsulation of the Itrybe dye grants an important precondition for the tracking of nanomaterial within living systems.

\section{Conclusion}

Our results show that $100 \mathrm{~nm}$ Itrybe-NPs are a highly suitable tool for distinguishing between healthy mice and mice with allergic lung inflammation in vivo and, subsequently, localizing NP-loaded immune cells by ex-vivo NIRF microscopy. Our approach may facilitate a broader understanding of the cellular and molecular events in lung disease and may be used as a model to assess new lung disease therapies, in particular nanotherapeutics.

\section{Materials and Methods}

\section{Materials and cells}

$100 \mathrm{~nm}$ carboxy-modified polystyrene particles were purchased from Kisker Biotech $\mathrm{GmbH}$ and stained with Itrybe using a previously described staining procedure. $^{37}$ All particles were ultrasonically treated prior to use. Itrybe was obtained from Otava Ltd. and used as received.

For in vitro experiments, $5 \times 10^{5}$ cells of the immortalized and adherent mouse alveolar cell line 
MH-S (CRL-2019, ATCC) ${ }^{64}$ were grown on $25 \mathrm{~mm}$ Poly-L-Lysin coated coverslips in complete RPMI medium, supplemented with $10 \%$ FCS and $0.05 \mathrm{mM} 2-$ mercaptoethanol. MH-S cells were incubated for $24 \mathrm{~h}$ with $100 \mathrm{ng} / \mathrm{ml}$ of Itrye-NPs either at $37^{\circ} \mathrm{C}$ or at $4^{\circ} \mathrm{C}$, washed twice with phosphate-buffered saline (PBS) and then fixed for $10 \mathrm{~min}$ at RT with $4 \%$ paraformaldehyde (PFA) before counterstaining the nuclei with $4 \mu \mathrm{g} / \mathrm{ml}$ DAPI and visualization using an Axiovert $200 \mathrm{M}$ inverted microscope (Carl Zeiss Microscopy $\mathrm{GmbH}$ ).

\section{Nanoparticle characterization}

The size distribution was analyzed by NTA using a NanoSight LM14 equipped with a $405 \mathrm{~nm}$ laser diode and scientific CMOS camera from Malvern Instruments GmbH. The aqueous suspensions containing about $5 \times 10^{8}$ particles $/ \mathrm{ml}$ were measured 5 times for $60 \mathrm{~s}$ and analyzed with the NanoSight NTA 2.3 Analytical Software Suite.

For the characterization of the morphology, Itrybe-NPs were dried on a silica substrate and imaged with an Agilent 5500 atomic force microscope (AFM) in the tapping mode using soft tapping cantilevers with a spring constant of nominally $5 \mathrm{~N} / \mathrm{m}$ and a tip radius of $<10 \mathrm{~nm}$ (Tap150Al-G from BudgetSensors).

Absorption measurements of $0.1 \quad \mathrm{w} \%$ particle suspensions were carried out with a Cary 5000 spectrophotometer from Varian Inc. Fluorescence measurements were performed with a calibrated Spectronics Instruments 8100 spectrofluorometer (Aminco Bowmann) equipped with Glan Thompson polarizers. The excitation polarizer was set to $0^{\circ}$ and the emission polarizer to $54.7^{\circ}$. The resulting emission spectra were corrected for the spectral responsivity of the fluorometer's emission channel.

\section{Animals}

Pathogen free female immunocompetent and hairless SKH-1mice (Crl: SKH1-Hr ${ }^{h r}$, 6-8 weeks of age, were purchased from Charles River Laboratories Inc. All animals were housed in a controlled environment at a regular 12-hour dark:light cycle, at $22^{\circ} \mathrm{C}$ and were fed laboratory chow and tap water ad libitum. Seven days prior to imaging experiments the standard food was replaced by chlorophyll free chow (Scientific Animal Food \& Engineering) to reduce autofluorescence from the stomach and gut of the animals. All animal experiments were performed in accordance with German animal ethics regulations and were approved by the local ethics office (Niedersächsisches Landesamt für Verbraucherschutz und
Lebensmittelsicherheit, LAVES, ethics approval no. 33.12-42502-04-12/0834).

\section{Induction of $A A I$}

On days 0 and 14 mice were immunized via intraperitoneal (i.p.) injection with $50 \mu \mathrm{g}$ OVA (Hyglos) and 0.5\% aluminium hydroxide gel adjuvant (Invivogen) in a volume of $0.2 \mathrm{ml} \mathrm{PBS}$, as well as intranasally (i.n.) with $50 \mu \mathrm{g}$ OVA in $25 \mu \mathrm{l}$ of PBS. On days 28, 29, 30 and 33 post-immunization, mice were challenged i.n. with $250 \mu \mathrm{g}$ OVA in $25 \mu \mathrm{l}$ PBS to induce an acute reaction. Control mice received PBS instead of OVA for all described steps except for the last two challenges on days 30 and 33, where they received $250 \mu \mathrm{g}$ OVA in $25 \mu \mathrm{l}$ PBS. Itrybe-NPs were given i.n. one day post challenges and mice were scanned over a given period of time by optical imaging. All i.n. applications were performed under short $2 \%$ isoflurane, $2 \mathrm{l} / \mathrm{min}$ oxygen anesthesia. Mice were sacrificed by $\mathrm{CO}_{2}$ overdose after the last scan.

\section{In vivo fluorescence reflectance imaging (FRI)}

Two-dimensional in vivo FRI was performed with an Optix MX2 System (ART), which comprises an interface for inhalation anesthetics and 4 pulsed laser diodes $(635,670,730$ and $785 \mathrm{~nm})$ as described before. $^{65}$ The Optix MX2 system works in a raster mode, illuminating each raster point with a multitude of short laser pulses $(<150 \mathrm{ps}$, repetition rate $8 \mathrm{MHz}$ ). For measurement of the emitted fluorescence light a single photon counting detector is used that, being triggered with each excitation pulse, records the amount of detected fluorescence photons and the time delay for the first detection event. From these values a time-of-flight histogram is created for each raster point (temporal point spread function, TPSF) which contains information about fluorescence intensity, concentration and depth of the fluorophore in the analyzed subject as well as the fluorescence lifetime. The lifetime is an additional parameter characterizing the fluorophore, which can be used to discriminate between probe specific signals and unwanted fluorescence derived from autofluorescence. Raster point separation is possible between $0.5-2 \mathrm{~mm}$, and photon collection time per raster point can be modified (integration time). Whereas the first allows for a more detailed analysis, increasing the integration time improves the statistics in the TPSF and allows for a more precise calculation of the underlying lifetime.

During in vivo scans, mice were anaesthetized by inhalation with $2 \%$ isoflurane, $21 /$ min oxygen for 15 20 min per scan. OVA challenged and control mice were prescanned to assess autofluorescence of the 
animals. One day post challenges the animals were given $160 \mu \mathrm{g}$ of $100 \mathrm{~nm}$ Itrybe-NPs in $30 \mu \mathrm{l}$ of PBS by i.n. application and were then scanned after $1 \mathrm{~h}, 5 \mathrm{~h}$ and $24 \mathrm{~h}$ ( $\mathrm{n}=16$ for AAI; $\mathrm{n}=15$ for controls). Itrybe fluorescence was measured using an excitation of 635 $\mathrm{nm}$ in combination with a $670 \mathrm{~nm}$ band-pass emission filter with $40 \mathrm{~nm}$ bandwidth. Scans were performed with a $1.5 \mathrm{~mm}$ (whole body scans) or $1.0 \mathrm{~mm}$ (lung scans) raster, a photon collection time (integration time) of $0.3-1 \mathrm{~s}$ per scan point and varying laser power. Intensity data and fluorescence lifetime were analyzed with the OptiView-2-02-00 software (ART).

Fluorescence intensity data are displayed in normalized counts (NC), where the measured fluorescence intensities (counts) were normalized for varying laser power and integration times, allowing for comparison of measurements with different settings. Data were quantified as average fluorescence intensity over a chosen region of interest over the lung and subsequently corrected for autofluorescence by subtracting the average fluorescence intensity from the same region of interest in the respective prescans.

Following imaging, mice were sacrificed and lungs were harvested for ex vivo scans and then cryofrozen for later microscopy. Bronchoalveolar lavage (BAL) was performed with some mice before harvesting of the lung.

\section{Bronchoalveolar lavage}

Five - $72 \mathrm{~h}$ after NP application, mice were sacrificed by $\mathrm{CO}_{2}$ overdose. BAL was performed by washing the airways gently three times with $500 \mu \mathrm{l}$ of $2 \%$ FCS/PBS after exposing and cannulating the trachea. Volumes were pooled and then washed once in the same buffer. Recovered cells were counted in a haemocytometer and $3 \times 10^{4}$ cells were used for obtaining cytospins with a Shandon Cytospin 4 (Thermo Scientific), followed by Giemsa staining (Sigma Aldrich) for differential cell counting. Where indicated, cytospins were fixed with $1: 1$ acetone/methanol solution, immunostained and counterstained with DAPI (4 $\mu \mathrm{g} / \mathrm{ml}$ ) for visualization of nuclei.

\section{Immunofluorescence staining}

Explanted lungs were cannulated and filled with $600 \mu \mathrm{l}$ of Tissue Tek OCT compound (Sakura Finetek) and immediately frozen in liquid nitrogen at $-80^{\circ} \mathrm{C}$. Frozen lung sections of $5 \mu \mathrm{m}$ or $20 \mu \mathrm{m}$ from AAI and control mice were obtained by cutting the lungs with a Jung Frigocut 2800E cryostat microtome (Leica Microsystems).
For NIRF microscopy, all sections were stained with DAPI $(4 \mu \mathrm{g} / \mathrm{ml})$ for visualization of nuclei. For detection of macrophages $5 \mu \mathrm{m}$ cryosections were fixed with acetone, stained with rat-anti-mouse CD68 antibody (clone FA-11, Abcam,1:300), followed by incubation with biotinylated goat-anti-rat antibody (BioLegend, 1:200) and Streptavidin-Alexa Fluor 555 (Life Technologies, 1:400). CD11c cell expression was assessed with Alexa Fluor 488 conjugated anti-mouse CD11c (clone N418, BioLegend, 1:200) and MHCII expression with Alexa Fluor 488 conjugated rat-antimouse MHCII antibody (clone M5/114.15.2, Biolegend, 1:200). ECF-L was stained with goat antimouse ECF-L antibody (R\&D, 1:150), followed by donkey anti-goat-Alexa Fluor 488 secondary antibody (Abcam, 1:400). AT1 cells were detected with hamster-anti-mouse podoplanin (Santa Cruz Biotechnology, 1:200), followed by goat anti-hamsterAlexa Fluor 488 secondary antibody (Life Technologies, 1:400). AT2 cells were detected by rabbit anti-mouse proS-PC antibody (Millipore, 1:2000), followed by goat anti-rabbit Alexa Fluor 488 (Life Technologies, 1:400). Images were acquired with an Axiovert $200 \mathrm{M}$ inverted microscope (Carl Zeiss Microscopy $\mathrm{GmbH}$ ) equipped with a Xenon lamp and a high sensitivity ORCA-AG digital camera (Hamamatsu). Image generation and processing were performed with the AxioVision Rel.4.6 software (Carl Zeiss Microscopy $\mathrm{GmbH}$ ) and FIJI (National Institutes of Health, Bethesda, USA). ${ }^{66}$

For quantification of NP-positive macrophages in AAI and control lungs $20 \mu \mathrm{m}$ sections were stained with anti-mouse CD68 antibody as described above. Representative $5.0 \times 2.8 \times 0.032 \mathrm{~mm}$ areas of the lung containing nuclei, CD68- and Itrybe-NP-positive cells were acquired by scanning and then merging 108 overlapping z-stacks with a Leica SP5 confocal laser scanning microscope (CLSM, Leica Microsystems) equipped with a 20x immersion objective (HCX PL APO CS, 0.70NA), a motorized stage, a tunable laser (470-670 nm) and hybrid detectors. DAPI, Alexa Fluor 555 and Itrybe-NPs were excited with 405, 555 and $650 \mathrm{~nm}$, respectively, and their individual emissions collected at 420-500 nm, 560-620 nm and 665-795 nm. Imaris 7.7.1 (Bitplane) was then used to visualize the fluorescence signal and generate ellipsoid spots for all nuclei, CD68- and Itrybe-positive cells based on the original CLSM multi-channel images. Briefly, threedimensional objects were generated for each nucleus, CD68 and Itrybe-positive cell and counted. To improve accuracy, each of the signal sources were modelled after an ellipsoid shape, whose major axis was parallel to the z-axis, so as to account for the lower z-axial optical resolution. The size of the ellipsoid model shape varied proportionally with 
respect to the size of the detected fluorescence cluster. Ellipsoids representing CD68 or Itrybe-NPs were considered to colocalize, when the distance of their center coordinates was less than $0.5 \mu \mathrm{m}$. Further visualization was conducted with the FIJI image processing package, while data analysis was done with Microsoft Excel. To account for differences in the total number of cells between AAI and control lungs, $\mathrm{CD}^{+} 8^{+} / \mathrm{NP}+$ colocalization was normalized to the total cell number measured as DAPI stained nuclei.

\section{Determination of OVA-specific antibodies by ELISA}

Sera were obtained $48 \mathrm{~h}$ after the last challenge. Serum levels of OVA-specific IgE and IgG1 were measured by ELISA. Briefly, 96-well plates (Nunc) were coated with $100 \mu \mathrm{g} / \mathrm{ml}$ OVA. After addition of serum samples, OVA specific $\operatorname{IgE}$ was detected with a commercial kit (Biolegend) according to the manufacturer's protocol and OVA specific IgG1 was detected with a biotinylated anti-IgG1 antibody (Southern Biotechnology) and amplified with Streptavidin HRP (Southern Biotechnology) as described before. ${ }^{67}$ Optical densities of the samples were measured at $450 \mathrm{~nm}$.

Determination of cytokine $m R N A$ expression by quantitative reverse transcription $P C R$

Pieces of cryofrozen lung tissue were homogenized with a tissue homogenizer and total RNA extracted with an RNAeasy Universal Plus kit (Qiagen) according to the manufacturer's protocol. RNA concentrations and purity were measured with a NanoDrop (Thermo Scientific). The SuperScript ${ }^{\circledR}$ First-Strand Synthesis System for RT-PCR (Life Technologies) was used to synthesize first-strand cDNA. Real-time PCR was performed using the QuantiTect SYBR Green PCR Kit (Qiagen) and a LightCycler 480II (Roche Diagnostics). Data were expressed as the calculated ratio of the values obtained for the specific cytokines vs the values of the housekeeping gene HPRT1. Forward and reverse primers for all genes are listed in Supplementary Table 1 .

\section{Histology}

For demonstration of AAI induction in SKH-1mice, AAI and control lungs were explanted 2 days after the last challenge, fixed in $10 \%$ buffered formalin and embedded in paraffin. $3 \mu \mathrm{m}$ sections were cut and H\&E staining was performed to evaluate the degree of lung infiltrating mononuclear cells. PAS staining was performed with a PAS staining kit according to the manufacturer's protocol (Merck) to show excess mucus production in paraffin sections from AAI mice.

\section{Statistical analysis}

Statistical analysis for significant difference of the mean values between two groups was performed with PAST ${ }^{68}$ using a Welch t-test. P-values $<0.05$ were considered significant. Cluster analysis was performed with PAST ${ }^{68}$ and calculated clusters were manually encircled in the scatter plot created by Microsoft Excel. Pearson's Correlation Coefficient was calculated by Microsoft Excel.

\section{Acknowledgements}

We thank S. Greco, R. Streich, S. Wolfgramm, B. Heidrich, H. Puchala and U. Kutzke for excellent help with the animal models, histology, immunofluorescence, cell culture and real-time PCR. We also thank R.M. Schürmann and I. Bald (BAM) for the conduction of the AFM measurements.

The presented data were funded in part by the European Commission through an FP7- IAPP Marie Curie Action (P3AGI: public private partnership for asthma imaging and genomics, GA 230739). 


\section{Supplementary Information Available}

Supplementary Figure 1-4

Supplementary Table 1

This material is available free of charge via the Internet at http://pubs.acs.org.

\section{References}

(1) Landfester, K. Miniemulsions for Nanoparticle Synthesis. In Colloid Chemistry II; Antonietti, P. D. M., Ed.; Topics in Current Chemistry; Springer Berlin Heidelberg, 2003; pp. 75-123.

(2) Sapsford, K. E.; Algar, W. R.; Berti, L.; Gemmill, K. B.; Casey, B. J.; Oh, E.; Stewart, M. H.; Medintz, I. L. Functionalizing Nanoparticles with Biological Molecules: Developing Chemistries That Facilitate Nanotechnology. Chem. Rev. 2013, 113, 1904-2074.

(3) Mu, Q.; Jiang, G.; Chen, L.; Zhou, H.; Fourches, D.; Tropsha, A.; Yan, B. Chemical Basis of Interactions Between Engineered Nanoparticles and Biological Systems. Chem. Rev. 2014, 114, 7740-7781.

(4) Liu, Q.-H.; Liu, J.; Guo, J.-C.; Yan, X.-L.; Wang, D.-H.; Chen, L.; Yan, F.-Y.; Chen, L.-G. Preparation of Polystyrene Fluorescent Microspheres Based on Some Fluorescent Labels. J. Mater. Chem. 2009, 19, 2018-2025.

(5) Lewinski, N.; Colvin, V.; Drezek, R. Cytotoxicity of Nanoparticles. Small Weinh. Bergstr. Ger. 2008, 4, $26-49$.

(6) Albanese, A.; Tang, P. S.; Chan, W. C. W. The Effect of Nanoparticle Size, Shape, and Surface Chemistry on Biological Systems. Annu. Rev. Biomed. Eng. 2012, 14, 1-16.

(7) Clift, M. J. D.; Rothen-Rutishauser, B.; Brown, D. M.; Duffin, R.; Donaldson, K.; Proudfoot, L.; Guy, K.; Stone, V. The Impact of Different Nanoparticle Surface Chemistry and Size on Uptake and Toxicity in a Murine Macrophage Cell Line. Toxicol. Appl. Pharmacol. 2008, 232, 418-427.

(8) Fröhlich, E.; Salar-Behzadi, S. Toxicological Assessment of Inhaled Nanoparticles: Role of in Vivo, Ex Vivo, in Vitro, and in Silico Studies. Int. J. Mol. Sci. 2014, 15, 4795-4822.

(9) Fröhlich, E.; Meindl, C.; Roblegg, E.; Griesbacher, A.; Pieber, T. R. Cytotoxity of Nanoparticles Is Influenced by Size, Proliferation and Embryonic Origin of the Cells Used for Testing. Nanotoxicology 2012, 6, 424-439.

(10) Xia, T.; Kovochich, M.; Liong, M.; Zink, J. I.; Nel, A. E. Cationic Polystyrene Nanosphere Toxicity Depends on CellSpecific Endocytic and Mitochondrial Injury Pathways. ACS Nano 2008, 2, 85-96.

(11) Jain, R. K.; Stylianopoulos, T. Delivering Nanomedicine to Solid Tumors. Nat. Rev. Clin. Oncol. 2010, 7, $653-664$.

(12) Aitken, R. J.; Chaudhry, M. Q.; Boxall, A. B. A.; Hull, M. Manufacture and Use of Nanomaterials: Current Status in the UK and Global Trends. Occup. Med. Oxf. Engl. 2006, 56, 300-306.

(13) Drescher, D.; Orts-Gil, G.; Laube, G.; Natte, K.; Veh, R. W.; Österle, W.; Kneipp, J. Toxicity of Amorphous Silica Nanoparticles on Eukaryotic Cell Model Is Determined by Particle Agglomeration and Serum Protein Adsorption Effects. Anal. Bioanal. Chem. 2011, 400, 1367-1373.

(14) Schipper, M. L.; Iyer, G.; Koh, A. L.; Cheng, Z.; Ebenstein, Y.; Aharoni, A.; Keren, S.; Bentolila, L. A.; Li, J.; Rao, J.; et al. Particle Size, Surface Coating, and PEGylation Influence the Biodistribution of Quantum Dots in Living Mice. Small Weinh. Bergstr. Ger. 2009, 5, 126-134.

(15) Bertrand, N.; Wu, J.; Xu, X.; Kamaly, N.; Farokhzad, O. C. Cancer Nanotechnology: The Impact of Passive and Active Targeting in the Era of Modern Cancer Biology. Adv. Drug Deliv. Rev. 2014, 66, 2-25.

(16) Daldrup-Link, H. E.; Golovko, D.; Ruffell, B.; Denardo, D. G.; Castaneda, R.; Ansari, C.; Rao, J.; Tikhomirov, G. A.; Wendland, M. F.; Corot, C.; et al. MRI of Tumor-Associated Macrophages with Clinically Applicable Iron Oxide Nanoparticles. Clin. Cancer Res. Off. J. Am. Assoc. Cancer Res. 2011, 17, 5695-5704.

(17) Gadde, S.; Even-Or, O.; Kamaly, N.; Hasija, A.; Gagnon, P. G.; Adusumilli, K. H.; Erakovic, A.; Pal, A. K.; Zhang, X.-Q.; Kolishetti, N.; et al. Development of Therapeutic Polymeric Nanoparticles for the Resolution of Inflammation. Adv. Healthc. Mater. 2014, 3, 1448-1456.

(18) Hoffmann, K.; Behnke, T.; Drescher, D.; Kneipp, J.; Resch-Genger, U. Near-Infrared-Emitting Nanoparticles for LifetimeBased Multiplexed Analysis and Imaging of Living Cells. ACS Nano 2013, 7, 6674-6684.

(19) Key, J.; Leary, J. F. Nanoparticles for Multimodal in Vivo Imaging in Nanomedicine. Int. J. Nanomedicine 2014, 9, 711726.

(20) Hellebust, A.; Richards-Kortum, R. Advances in Molecular Imaging: Targeted Optical Contrast Agents for Cancer Diagnostics. Nanomed. 2012, 7, 429-445.

(21) Behnke, T.; Mathejczyk, J. E.; Brehm, R.; Würth, C.; Gomes, F. R.; Dullin, C.; Napp, J.; Alves, F.; Resch-Genger, U. Target-Specific Nanoparticles Containing a Broad Band Emissive NIR Dye for the Sensitive Detection and Characterization of Tumor Development. Biomaterials 2013, 34, 160-170.

(22) Clift, M. J. D.; Rothen-Rutishauser, B.; Brown, D. M.; Duffin, R.; Donaldson, K.; Proudfoot, L.; Guy, K.; Stone, V. The Impact of Different Nanoparticle Surface Chemistry and Size on Uptake and Toxicity in a Murine Macrophage Cell Line. Toxicol. Appl. Pharmacol. 2008, 232, 418-427.

(23) Olivier, V.; Duval, J. L.; Hindié, M.; Pouletaut, P.; Nagel, M. D. Comparative Particle-Induced Cytotoxicity toward Macrophages and Fibroblasts. Cell Biol. Toxicol. 2003, 19, 145-159.

(24) Gessner, A.; Lieske, A.; Paulke, B.-R.; Müller, R. H. Functional Groups on Polystyrene Model Nanoparticles: Influence on Protein Adsorption. J. Biomed. Mater. Res. A 2003, 65, 319-326. 
(25) Sun, X.; Rossin, R.; Turner, J. L.; Becker, M. L.; Joralemon, M. J.; Welch, M. J.; Wooley, K. L. An Assessment of the Effects of Shell Cross-Linked Nanoparticle Size, Core Composition, and Surface PEGylation on in Vivo Biodistribution. Biomacromolecules 2005, 6, 2541-2554.

(26) Behnke, T.; Würth, C.; Hoffmann, K.; Hübner, M.; Panne, U.; Resch-Genger, U. Encapsulation of Hydrophobic Dyes in Polystyrene Micro- and Nanoparticles via Swelling Procedures. J. Fluoresc. 2011, 21, 937-944.

(27) Balhara, J.; Gounni, A. S. The Alveolar Macrophages in Asthma: A Double-Edged Sword. Mucosal Immunol. 2012, 5, 605-609.

(28) Bates, J. H. T.; Rincon, M.; Irvin, C. G. Animal Models of Asthma. Am. J. Physiol. - Lung Cell. Mol. Physiol. 2009, 297, L401-L410.

(29) Blyth, D. I.; Pedrick, M. S.; Savage, T. J.; Hessel, E. M.; Fattah, D. Lung Inflammation and Epithelial Changes in a Murine Model of Atopic Asthma. Am. J. Respir. Cell Mol. Biol. 1996, 14, 425-438.

(30) Draijer, C.; Robbe, P.; Boorsma, C. E.; Hylkema, M. N.; Melgert, B. N. Characterization of Macrophage Phenotypes in Three Murine Models of House-Dust-Mite-Induced Asthma. Mediators Inflamm. 2013, 2013, 632049.

(31) Thepen, T.; Claassen, E.; Hoeben, K.; Brevé, J.; Kraal, G. Migration of Alveolar Macrophages from Alveolar Space to Paracortical T Cell Area of the Draining Lymph Node. Adv. Exp. Med. Biol. 1993, 329, 305-310.

(32) Willart, M. A. M.; Hammad, H. Alarming Dendritic Cells for Allergic Sensitization. Allergol. Int. Off. J. Jpn. Soc. Allergol. 2010, 59, 95-103.

(33) Gammon, S. T.; Foje, N.; Brewer, E. M.; Owers, E.; Downs, C. A.; Budde, M. D.; Leevy, W. M.; Helms, M. N. Preclinical Anatomical, Molecular, and Functional Imaging of the Lung with Multiple Modalities. Am. J. Physiol. - Lung Cell. Mol. Physiol. 2014, 306, L897-L914.

(34) Napp, J.; Mathejczyk, J. E.; Alves, F. Optical Imaging in Vivo with a Focus on Paediatric Disease: Technical Progress, Current Preclinical and Clinical Applications and Future Perspectives. Pediatr. Radiol. 2011, 41, 161-175.

(35) Weissleder, R.; Ntziachristos, V. Shedding Light onto Live Molecular Targets. Nat. Med. 2003, 9, $123-128$.

(36) Schaffer, B. S.; Grayson, M. H.; Wortham, J. M.; Kubicek, C. B.; McCleish, A. T.; Prajapati, S. I.; Nelon, L. D.; Brady, M. M.; Jung, I.; Hosoyama, T.; et al. Immune Competency of a Hairless Mouse Strain for Improved Preclinical Studies in Genetically Engineered Mice. Mol. Cancer Ther. 2010, 9, 2354-2364.

(37) Behnke, T.; Würth, C.; Laux, E.-M.; Hoffmann, K.; Resch-Genger, U. Simple Strategies towards Bright Polymer Particles via One-Step Staining Procedures. Dyes Pigments 2012, 94, 247-257.

(38) DeRose, P. C.; Resch-Genger, U. Recommendations for Fluorescence Instrument Qualification: The New ASTM Standard Guide. Anal. Chem. 2010, 82, 2129-2133.

(39) Kunisch, E.; Fuhrmann, R.; Roth, A.; Winter, R.; Lungershausen, W.; Kinne, R. W. Macrophage Specificity of Three AntiCD68 Monoclonal Antibodies (KP1, EBM11, and PGM1) Widely Used for Immunohistochemistry and Flow Cytometry. Ann. Rheum. Dis. 2004, 63, 774-784.

(40) Murray, P. J.; Wynn, T. A. Protective and Pathogenic Functions of Macrophage Subsets. Nat. Rev. Immunol. 2011, 11, 723-737.

(41) Bedoret, D.; Wallemacq, H.; Marichal, T.; Desmet, C.; Quesada Calvo, F.; Henry, E.; Closset, R.; Dewals, B.; Thielen, C.; Gustin, P.; et al. Lung Interstitial Macrophages Alter Dendritic Cell Functions to Prevent Airway Allergy in Mice. J. Clin. Invest. 2009, 119, 3723-3738.

(42) Lagranderie, M.; Nahori, M.-A.; Balazuc, A.-M.; Kiefer-Biasizzo, H.; Lapa e Silva, J.-R.; Milon, G.; Marchal, G.; Vargaftig, B. B. Dendritic Cells Recruited to the Lung Shortly after Intranasal Delivery of Mycobacterium Bovis BCG Drive the Primary Immune Response towards a Type 1 Cytokine Production. Immunology 2003, 108, 352-364.

(43) Zaynagetdinov, R.; Sherrill, T. P.; Kendall, P. L.; Segal, B. H.; Weller, K. P.; Tighe, R. M.; Blackwell, T. S. Identification of Myeloid Cell Subsets in Murine Lungs Using Flow Cytometry. Am. J. Respir. Cell Mol. Biol. 2013, 49, $180-189$.

(44) Geiser, M.; Rothen-Rutishauser, B.; Kapp, N.; Schurch, S.; Kreyling, W.; Schulz, H.; Semmler, M.; Hof, V. I.; Heyder, J.; Gehr, P. Ultrafine Particles Cross Cellular Membranes by Nonphagocytic Mechanisms in Lungs and in Cultured Cells. Environ. Health Perspect. 2005, 113, 1555-1560.

(45) Kirby, A. C.; Coles, M. C.; Kaye, P. M. Alveolar Macrophages Transport Pathogens to Lung Draining Lymph Nodes. J. Immunol. Baltim. Md 1950 2009, 183, 1983-1989.

(46) Markus, M. A.; Dullin, C.; Mitkovski, M.; Prieschl-Grassauer, E.; Epstein, M. M.; Alves, F. Non-Invasive Optical Imaging of Eosinophilia during the Course of an Experimental Allergic Airways Disease Model and in Response to Therapy. PloS One 2014, 9, e90017.

(47) Cortez-Retamozo, V.; Swirski, F. K.; Waterman, P.; Yuan, H.; Figueiredo, J. L.; Newton, A. P.; Upadhyay, R.; Vinegoni, C.; Kohler, R.; Blois, J.; et al. Real-Time Assessment of Inflammation and Treatment Response in a Mouse Model of Allergic Airway Inflammation. J. Clin. Invest. 2008, 118, 4058-4066.

(48) Biffi, S.; Dal Monego, S.; Dullin, C.; Garrovo, C.; Bosnjak, B.; Licha, K.; Welker, P.; Epstein, M. M.; Alves, F. Dendritic Polyglycerolsulfate Near Infrared Fluorescent (NIRF) Dye Conjugate for Non-Invasively Monitoring of Inflammation in an Allergic Asthma Mouse Model. PloS One 2013, 8, e57150.

(49) Varna, M.; Ratajczak, P.; Ferreira, I.; Leboeuf, C.; Bousquet, G.; Janin, A. In Vivo Distribution of Inorganic Nanoparticles in Preclinical Models. J. Biomater. Nanobiotechnology 2012, 3, 269.

(50) Beckmann, N.; Cannet, C.; Babin, A. L.; Blé, F.-X.; Zurbruegg, S.; Kneuer, R.; Dousset, V. In Vivo Visualization of Macrophage Infiltration and Activity in Inflammation Using Magnetic Resonance Imaging. Wiley Interdiscip. Rev. Nanomed. Nanobiotechnol. 2009, 1, 272-298. 
(51) Weissleder, R.; Nahrendorf, M.; Pittet, M. J. Imaging Macrophages with Nanoparticles. Nat. Mater. 2014, 13, $125-138$.

(52) Madsen, S. J.; Gach, H. M.; Hong, S. J.; Uzal, F. A.; Peng, Q.; Hirschberg, H. Increased Nanoparticle-Loaded Exogenous Macrophage Migration into the Brain Following PDT-Induced Blood-Brain Barrier Disruption. Lasers Surg. Med. 2013, $45,524-532$.

(53) Dullin, C.; Dal Monego, S.; Larsson, E.; Mohammadi, S.; Krenkel, M.; Garrovo, C.; Biffi, S.; Lorenzon, A.; Markus, A.; Napp, J.; et al. Functionalized Synchrotron in-Line Phase-Contrast Computed Tomography: A Novel Approach for Simultaneous Quantification of Structural Alterations and Localization of Barium-Labelled Alveolar Macrophages within Mouse Lung Samples. J. Synchrotron Radiat. 2015, 22, 143-155.

(54) Geiser, M.; Wigge, C.; Conrad, M. L.; Eigeldinger-Berthou, S.; Künzi, L.; Garn, H.; Renz, H.; Mall, M. A. Nanoparticle Uptake by Airway Phagocytes after Fungal Spore Challenge in Murine Allergic Asthma and Chronic Bronchitis. BMC Pulm. Med. 2014, 14, 116.

(55) Dames, P.; Gleich, B.; Flemmer, A.; Hajek, K.; Seidl, N.; Wiekhorst, F.; Eberbeck, D.; Bittmann, I.; Bergemann, C.; Weyh, T.; et al. Targeted Delivery of Magnetic Aerosol Droplets to the Lung. Nat. Nanotechnol. 2007, 2, 495-499.

(56) Boorsma, C. E.; Draijer, C.; Melgert, B. N. Macrophage Heterogeneity in Respiratory Diseases. Mediators Inflamm. 2013, 2013, 769214.

(57) Robbe, P.; Draijer, C.; Borg, T. R.; Luinge, M.; Timens, W.; Wouters, I. M.; Melgert, B. N.; Hylkema, M. N. Distinct Macrophage Phenotypes in Allergic and Nonallergic Lung Inflammation. Am. J. Physiol. Lung Cell. Mol. Physiol. 2014, ajplung.00341.2014.

(58) Sun, X.; Jones, H. P.; Dobbs, N.; Bodhankar, S.; Simecka, J. W. Dendritic Cells Are the Major Antigen Presenting Cells in Inflammatory Lesions of Murine Mycoplasma Respiratory Disease. PLoS ONE 2013, 8, e55984.

(59) John-Schuster, G.; Hager, K.; Conlon, T. M.; Irmler, M.; Beckers, J.; Eickelberg, O.; Yildirim, A. Ö. Cigarette SmokeInduced iBALT Mediates Macrophage Activation in a B Cell-Dependent Manner in COPD. Am. J. Physiol. Lung Cell. Mol. Physiol. 2014.

(60) Takenaka, S.; Karg, E.; Kreyling, W. G.; Lentner, B.; Möller, W.; Behnke-Semmler, M.; Jennen, L.; Walch, A.; Michalke, B.; Schramel, P.; et al. Distribution Pattern of Inhaled Ultrafine Gold Particles in the Rat Lung. Inhal. Toxicol. 2006, 18, 733-740.

(61) Thorley, A. J.; Ruenraroengsak, P.; Potter, T. E.; Tetley, T. D. Critical Determinants of Uptake and Translocation of Nanoparticles by the Human Pulmonary Alveolar Epithelium. ACS Nano 2014, 8, 11778-11789.

(62) Gupta, N.; Hatoum, H.; Dy, G. K. First Line Treatment of Advanced Non-Small-Cell Lung Cancer - Specific Focus on Albumin Bound Paclitaxel. Int. J. Nanomedicine 2014, 9, 209-221.

(63) Jain, N. K.; Mishra, V.; Mehra, N. K. Targeted Drug Delivery to Macrophages. Expert Opin. Drug Deliv. 2013, 10, 353367.

(64) Mbawuike, I. N.; Herscowitz, H. B. MH-S, a Murine Alveolar Macrophage Cell Line: Morphological, Cytochemical, and Functional Characteristics. J. Leukoc. Biol. 1989, 46, 119-127.

(65) Dullin, C.; Zientkowska, M.; Napp, J.; Missbach-Guentner, J.; Krell, H.-W.; Müller, F.; Grabbe, E.; Tietze, L.-F.; Alves, F. Semiautomatic Landmark-Based Two-Dimensional-Three-Dimensional Image Fusion in Living Mice: Correlation of nearInfrared Fluorescence Imaging of Cy5.5-Labeled Antibodies with Flat-Panel Volume Computed Tomography. Mol. Imaging 2009, 8, 2-14.

(66) Schindelin, J.; Arganda-Carreras, I.; Frise, E.; Kaynig, V.; Longair, M.; Pietzsch, T.; Preibisch, S.; Rueden, C.; Saalfeld, S.; Schmid, B.; et al. Fiji: An Open-Source Platform for Biological-Image Analysis. Nat. Methods 2012, 9, 676-682.

(67) Reiner, D.; Lee, R.-Y.; Dekan, G.; Epstein, M. M. No Adjuvant Effect of Bacillus Thuringiensis-Maize on Allergic Responses in Mice. PLoS ONE 2014, 9.

(68) Hammer, Ø.; Harper, D. A. T. I Ryan, PD (2001) PAST: Paleontological Statistics Software Package for Education and Data Analysis. Palaeontol. Electron. 4, 9.

\section{Abbreviations}

AFM, atomic force microscopy; BAL, broncho-alveolar lavage; COPD, Chronic Obstructive Pulmonary Disease; CT, computed tomography; DAPI, 4',6-diamidino-2-phenylindole; FRI, fluorescence reflectance imaging; MRI, magnetic resonance imaging; NIRF, near infrared fluorescence; i.n., intranasal; NP, nanoparticles; NTA, nanoparticle tracking analysis; OCT, optical coherence tomography; OVA, ovalbumin; PET, positron emission tomography; RT, room temperature; SPECT, single-photon emission computed tomography; THF, tetrahydrofuran 

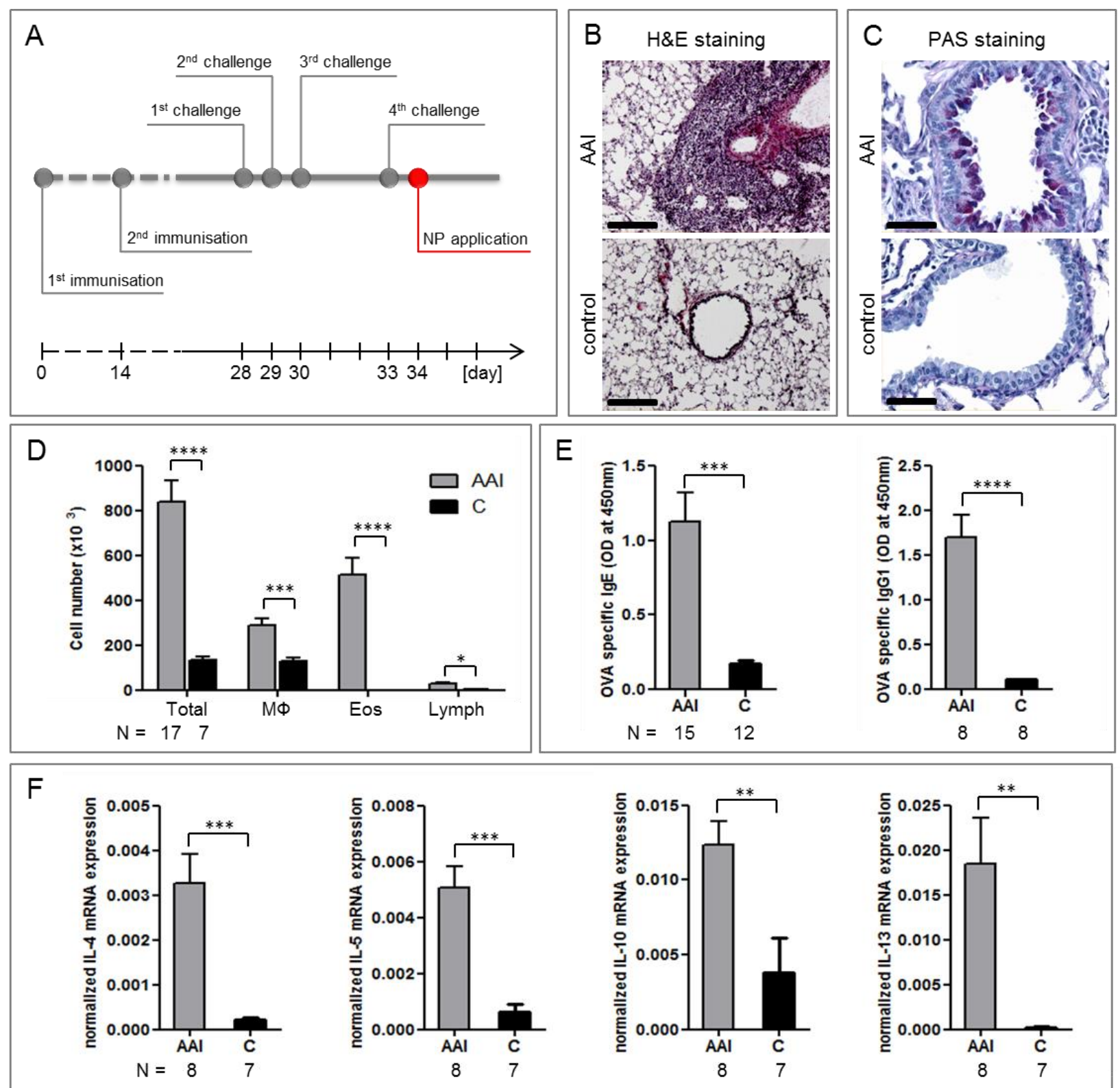

Figure 1. SKH-1 mice are susceptible to AAI induction. (A) General immunization and challenge scheme for the allergic inflammation model used in all investigations. (B) H\&E and (C) PAS staining of lung paraffin sections of AAI (upper panel) and control mice (lower panel). (B) AAI mice exhibit typical cell infiltration around blood vessels and bronchi, as well as (C) goblet cells filled with secretory granules (magenta) and increased goblet cell hyperplasia (upper panel). (D) Cell composition in BALs shows increased total cells, macrophages (MØ), eosinophils (Eos) and lymphocytes (Lymph) in AAI, (E) OVA-specific IgE and IgG1 are significantly increased in AAI. Serum was diluted 1:10 for IgE and 1:625 for IgG1, (F) Expression of IL-4, IL-5, IL-10 and IL-13 Cytokines expression in lung tissue is significantly elevated in AAI. Scale bars represent $200 \mu \mathrm{m}$ (B) and $50 \mu \mathrm{m}(\mathrm{C}){ }^{*}$ represents $\mathrm{p}<0.05$ 

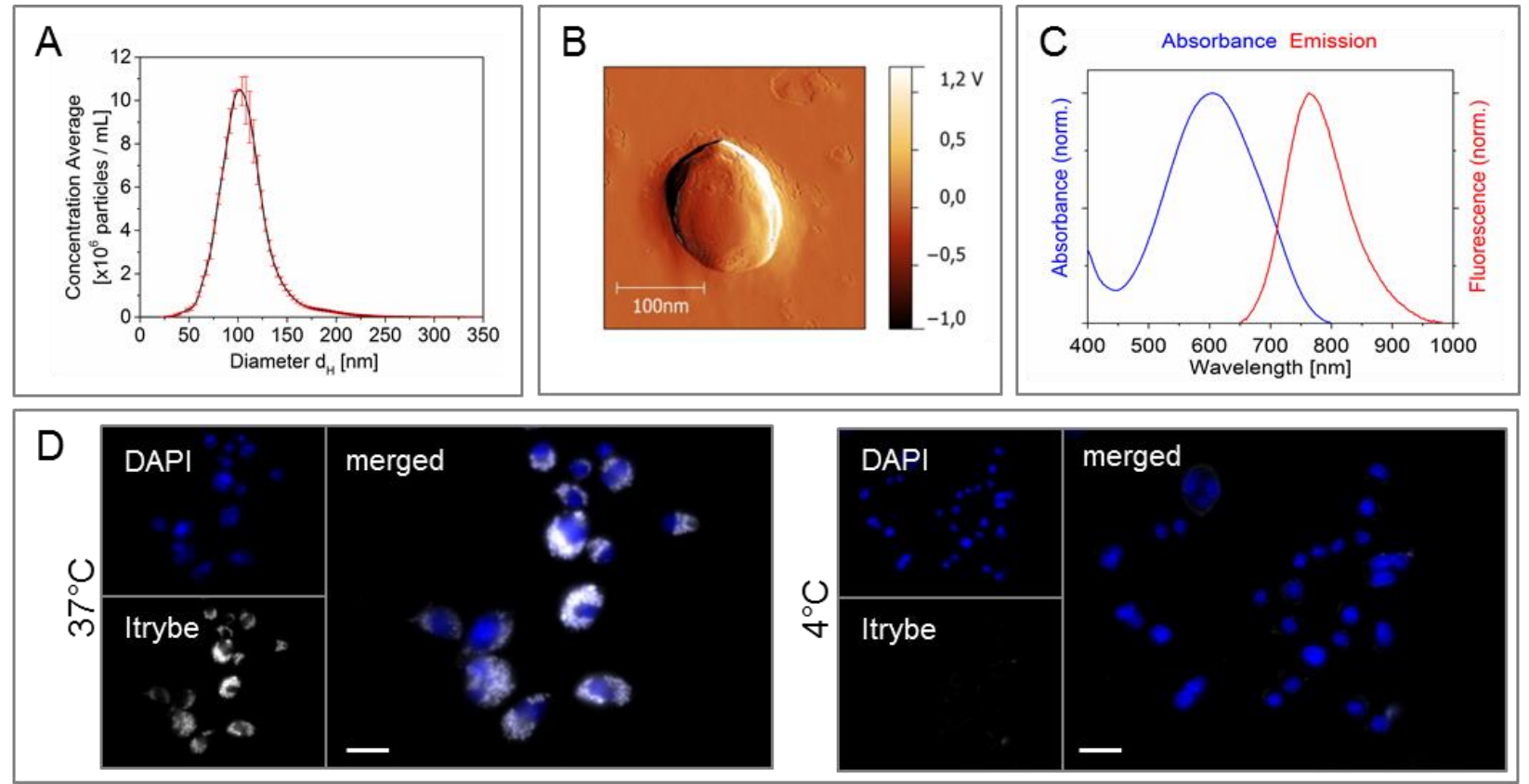

Figure 2. Itrybe-NP characteristic features. (A) Size distribution determined via NTA. (B) AFM tapping mode amplitude image of a single Itrybe-NP on silica substrate. (C) Itrybe-NP absorbance and emission spectra (excitation at $600 \mathrm{~nm}$ ). (D) In vitro uptake of Itrybe-NPs by $\mathrm{MH}-\mathrm{S}$ macrophages after $24 \mathrm{~h}$ incubation at $37^{\circ} \mathrm{C}$ (left) and uptake inhibition due to the inactivation of phagocytosis by switching the temperature from $37^{\circ} \mathrm{C}$ to $4^{\circ} \mathrm{C}$ (right). Scale bars in D represent $20 \mu \mathrm{m}$. 

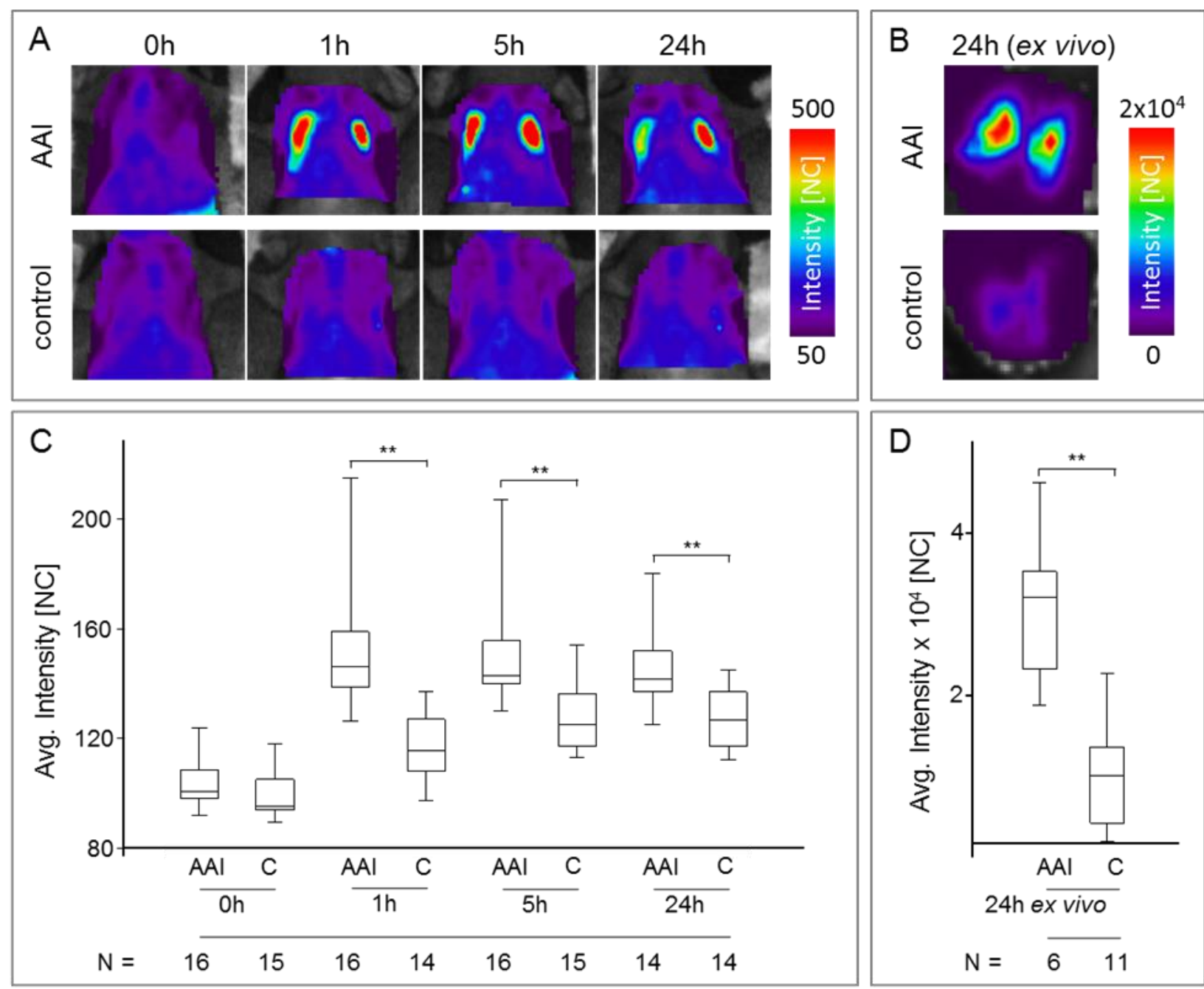

Figure 3. AAI mice demonstrate significantly higher intensities over the lungs than controls in vivo (A) and ex vivo (B). Fluorescence intensity distribution is displayed in normalized counts (NC). On average, AAI mice showed maximum intensities $5 \mathrm{~h}$ after i.n. Itrybe-NP application. In vivo (C) and ex vivo (D) fluorescence imaging results at the indicated time points after i.n. NP application are summarized in box plots of average fluorescence intensities over the lung area. $* * \mathrm{p}<0.05$ 

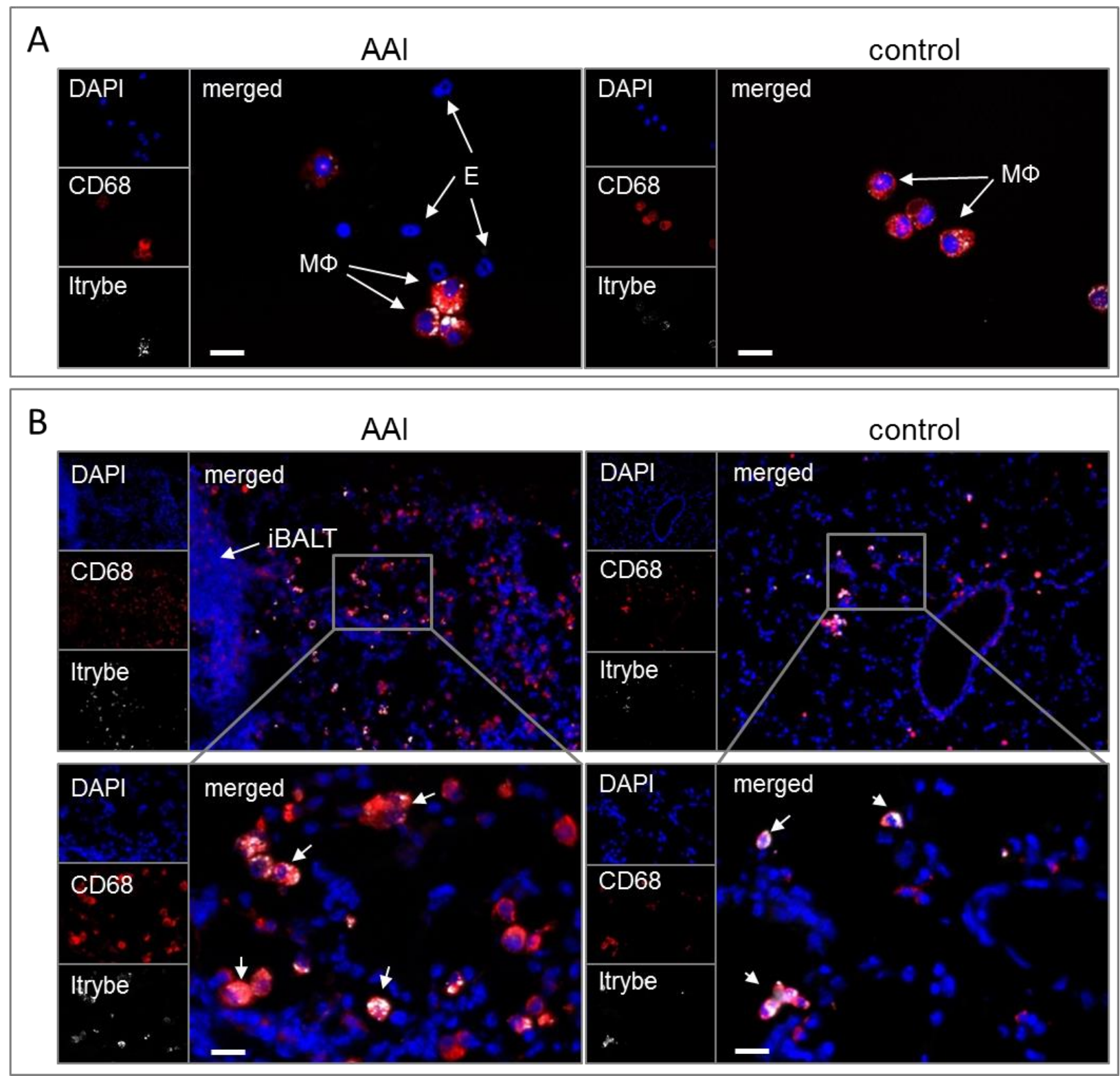

Figure 4. Itrybe-NPs are taken up by $\mathrm{CD} 68^{+}$cells. Anti-CD68 immunostaining of cytospins from BAL $24 \mathrm{~h}$ after i.n. Itrybe-NP instillation demonstrates uptake of NPs by CD68 ${ }^{+}$cells in both AAI (A, left panel) and control (A, right panel) mice. Anti-CD68 immunostaining of cryosections from lungs of AAI (B, upper panel) and control mice (B, lower panel) shows a higher amount of Itrybe-NP containing $\mathrm{CD}^{+} 8^{+}$cells (arrows) in AAI mice compared to controls as well as clustering of Itrybe-NP containing $\mathrm{CD}^{+} 8^{+}$around iBALT in AAI. Note, that Itrybe-positive cells are never found in iBALT. Lower panels in B show magnifications of the selected areas in the upper panels. CD68 staining is shown in red, Itrybe-NPs in white. DAPI (blue) was used to stain cell nuclei in all images. MØ: macrophages; E: eosinophils. Scale bars represent $20 \mu \mathrm{m}$. 

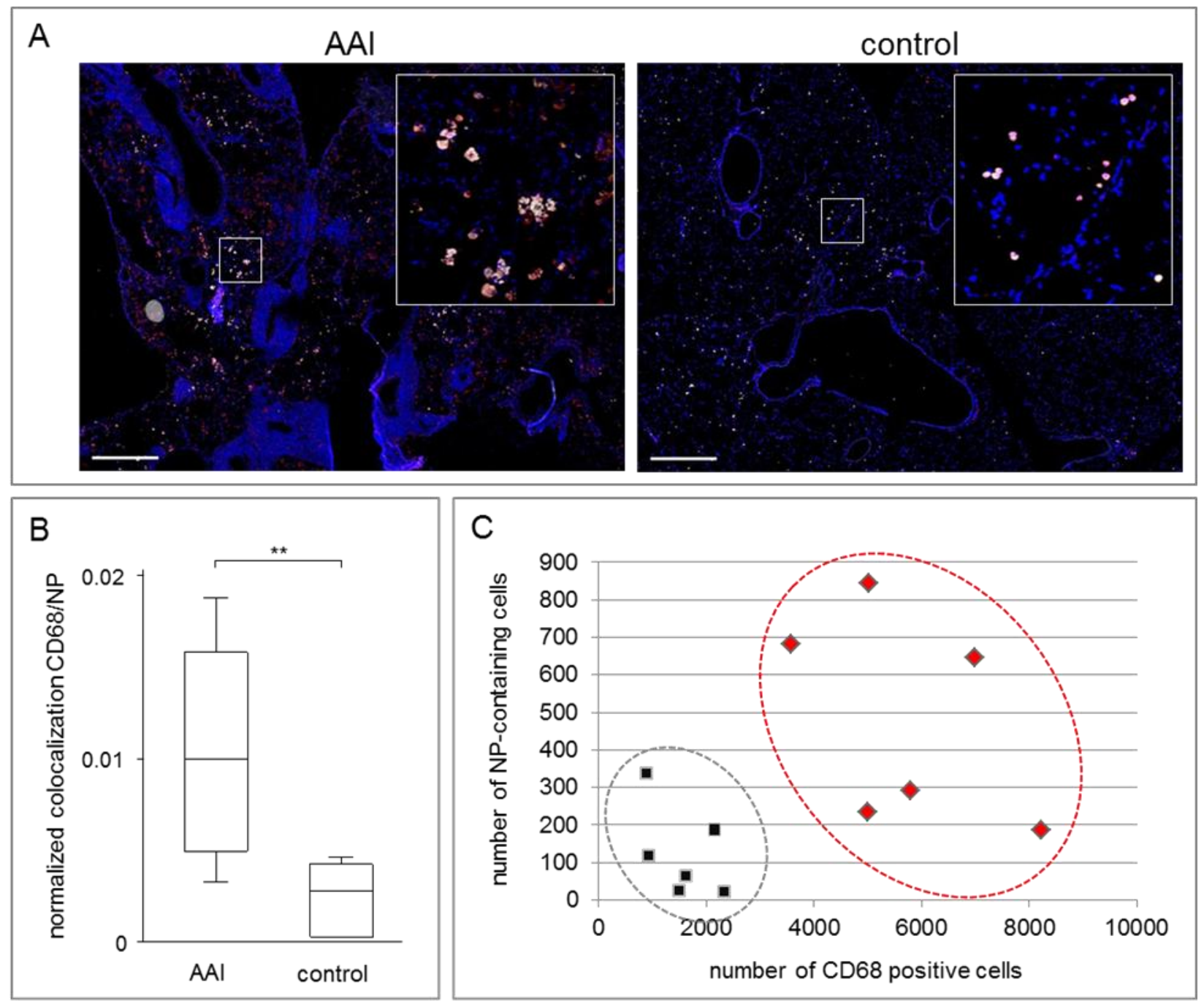

Figure 5. Distribution of Itrybe-NP-loaded AMs. (A) Representative stitched image of 12x9 tiles $(456 \times 456 \times 32 \mu \mathrm{m}$ each) selected in the center of an AAI and a control lung cryosection stained with anti-CD68 antibody showing an overview of Itrybe-NP distribution within the lung. (B) AAI lungs show a 4 times higher number of $\mathrm{NP}^{+} / \mathrm{CD} 68^{+}$cells than controls. Shown are colocalized $\mathrm{CD} 68+/ \mathrm{NP}+$ cells normalized to total nuclei. (C) The number of Itrybe-NP containing cells cluster for AAI (red) and controls (blue), as seen in the scatter plot of the total number of cells with colocalization of NP/CD68 from one tiled image each of 6 AAI and 6 control cryosections. DAPI was used to stain nuclei in all images. Scale bars in A represent $500 \mu \mathrm{m} . * *$ in B represents $\mathrm{p}<0.05$ 

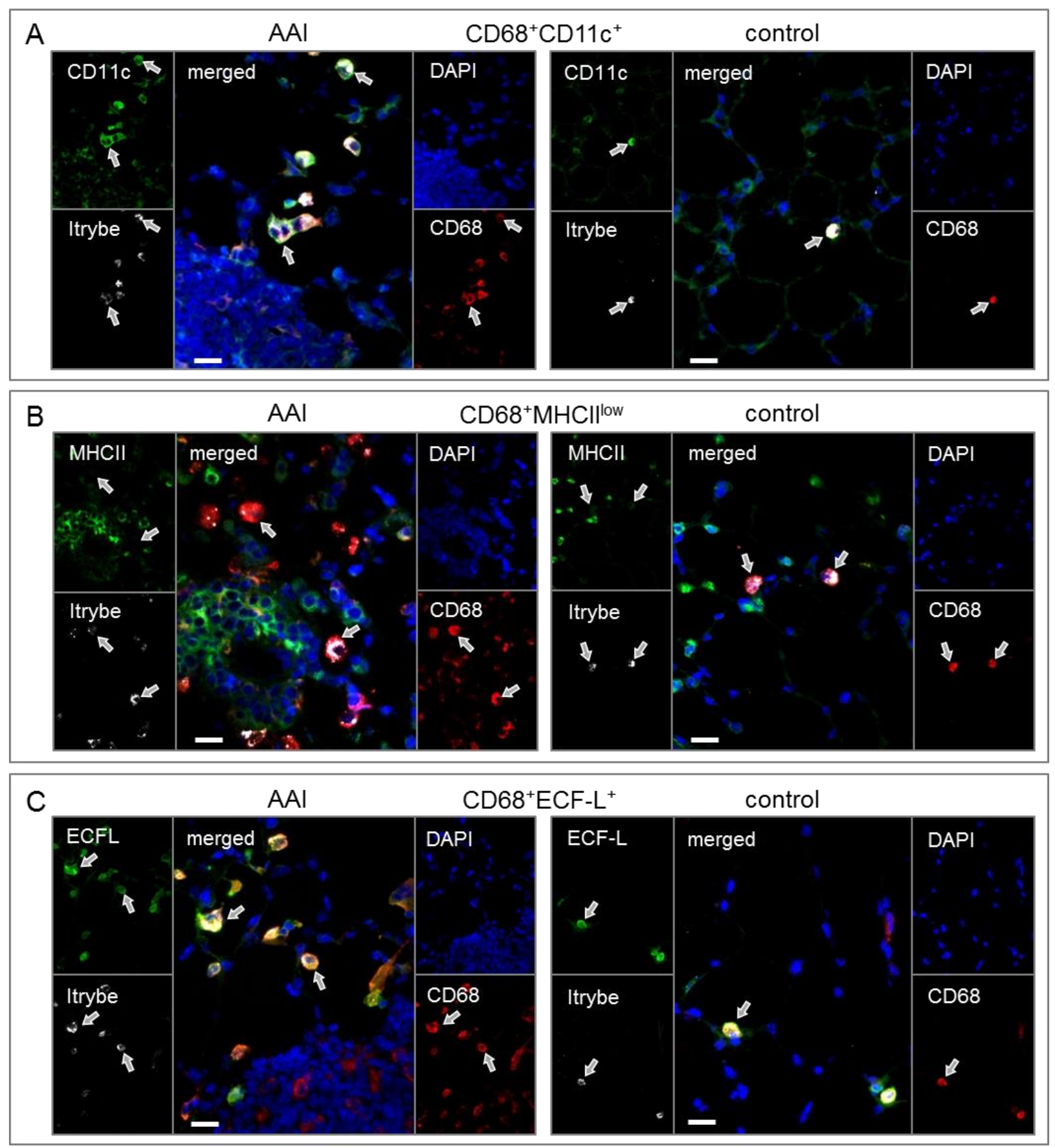

Figure 6. Itrybe-NPs are phagocytized by $\mathrm{CD}_{6} 8^{+} \mathrm{CD} 11 \mathrm{c}^{+} \mathrm{ECF}-\mathrm{L}^{+} \mathrm{MHCII}{ }^{\text {low }}$ alveolar $\mathrm{M} 2$ macrophages. Immunofluorescence microscopy of co-staining of AAI lung cryosections, $24 \mathrm{~h}$ after Itrybe-NP application with antibodies directed against CD68 and CD11c (A), MHCII (B) and ECF-L (C). Itrybe-NPs are shown in white; expression of CD68 is shown in red. Expression of CD11c, MHCII and ECF-L are shown in green. DAPI (blue) was used to stain nuclei in all images. Scale bars represent $20 \mu \mathrm{m}$. 


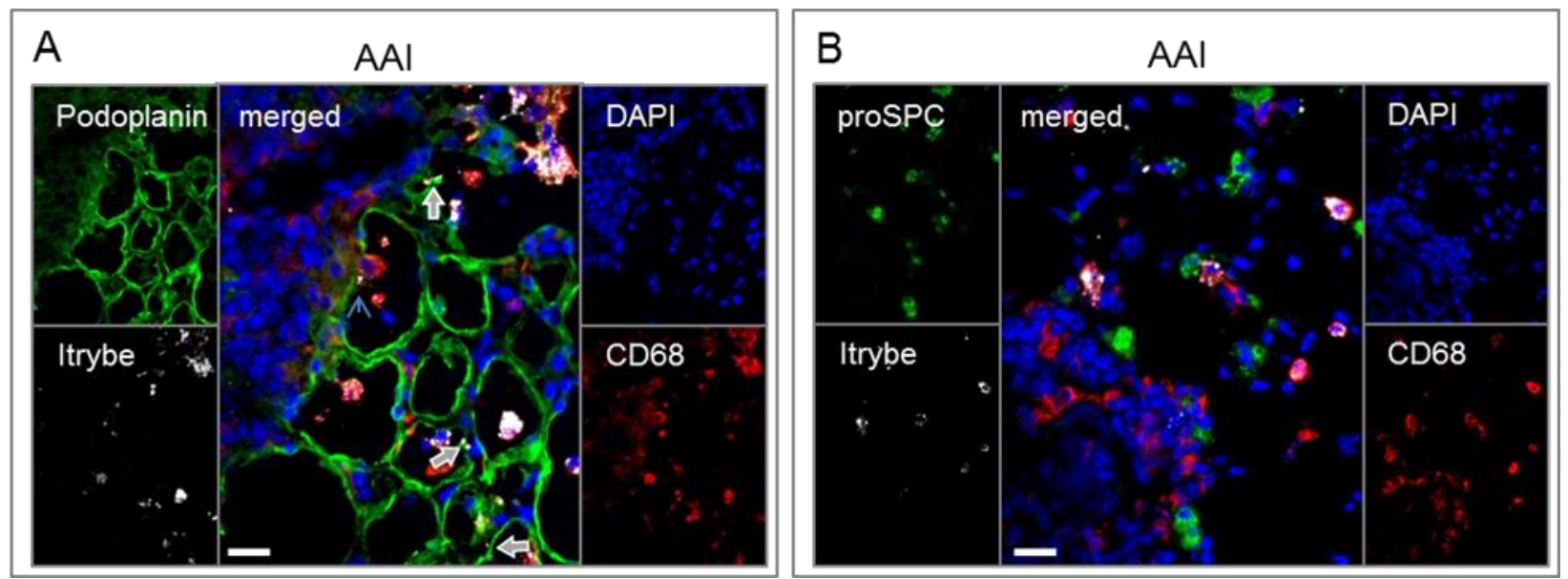

Figure 7. Occasional uptake of Itrybe-NPs by epithelial type I pulmonary cells. Co-staining of AAI lung cryosections with antibodies directed against CD68 and podoplanin (A) or CD68 and proSPC (B) $24 \mathrm{~h}$ after i.n. Itrybe-NP application. Few Itrybe-NPs were found in some podoplanin $^{+}$AT1 cells (A, arrows), but never in proSPC ${ }^{+}$AT2 cells. CD68 expression is shown in red; podoplanin and proSPC expression are in green and Itrybe-NPs are shown in white. DAPI (blue) was used to stain nuclei in all images. Scale bars represent $20 \mu \mathrm{m}$. 


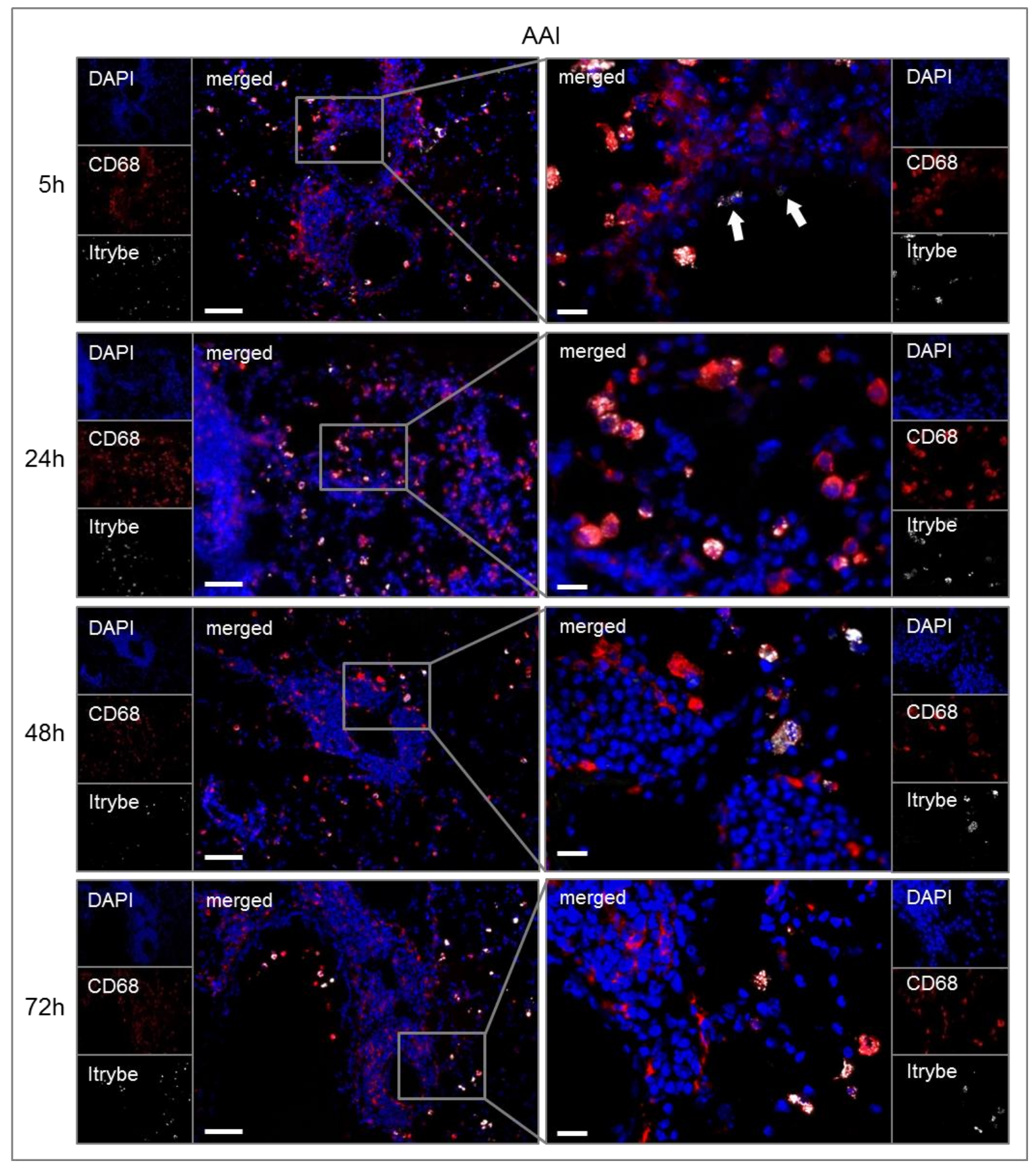

Figure 8. Itrybe-NP uptake by AMs in dependence of time. Immunostaining of AAI cryosections with anti-CD68 antibody. Five $h$ after i.n. Itrybe-NP application, many NPs are within alveoli and bronchioles, where they have not yet been phagocytosed by cells (inset, white arrows). Forty eight $\mathrm{h}$ and $72 \mathrm{~h}$ after i.n. NP application show a similar pattern of Itrybe-NP localization as $24 \mathrm{~h}$ with all NPs taken up by AMs. CD68 expression is shown in red; Itrybe-NPs are in white. DAPI (blue) was used to stain nuclei. Scale bars represent $100 \mu \mathrm{m}$ (left panel) or $20 \mu \mathrm{m}$ (right panel). 